\title{
SPECTRAL HALO FOR HILBERT MODULAR FORMS
}

\author{
RUFEI REN AND BIN ZHAO
}

\begin{abstract}
Let $F$ be a totally real field and $p$ be an odd prime which splits completely in $F$. We prove that the eigenvariety associated to a definite quaternion algebra over $F$ satisfies the following property: over a boundary annulus of the weight space, the eigenvariety is a disjoint union of countably infinitely many connected components which are finite over the weight space; on each fixed connected component, the ratios between the $U_{\mathfrak{p}}$-slopes of points and the $p$-adic valuations of the $\mathfrak{p}$-parameters are bounded by explicit numbers, for all primes $\mathfrak{p}$ of $F$ over $p$. Applying Hansen's $p$-adic interpolation theorem, we are able to transfer our results to Hilbert modular eigenvarieties. In particular, we prove that on every irreducible component of Hilbert modular eigenvarieties, as a point moves towards the boundary, its $U_{p}$ slope goes to zero. In the case of eigencurves, this completes the proof of Coleman-Mazur's 'halo' conjecture.
\end{abstract}

\section{Contents}

1. Introduction

2. Newton-Hodge decomposition over certain noncommutative rings 8

3. Automorphic forms for definite quaternion algebras and completed homology $\quad 26$

4. A filtration on the space of integral $p$-adic automorphic forms 41

5. Proof of the main theorem 53

6. Application to Hilbert modular eigenvarieties 61 References

\section{INTRODUCTION}

The theory of $p$-adic analytic families of modular forms grew out of the study of congruences of modular forms. In [24], Serre gave the first example of a $p$-adic family of modular forms-an Eisenstein family. After more than one decade, Hida made the first major step towards the construction of $p$-adic families of cuspforms. In [13] and [14], he constructed $p$-adic families of ordinary modular forms. His theory led Mazur to develop his general theory of deformations of Galois representations in [21, which turns out to be a crucial ingredient of Wiles' proof of Fermat's last theorem. The theory of $p$-adic families of modular forms reached a culmination in Coleman and Mazur's celebrated work [9] in which they constructed eigencurves. An eigencurve is a rigid analytic curve whose points correspond to finite slopes normalized overconvergent eigenforms. When pursuing a description of the geometry of the eigencurves, Coleman and Mazur raised the following question based on detailed computations for small primes: does the slope always tend to 0 as one approaches the 'boundary' of the weight space? This problem has been made into a much more delicate conjecture based on the computation of Buzzard and Kilford in [7]. It is expected that over

Date: April 21, 2021.

2010 Mathematics Subject Classification. 11T23 (primary), 11L07 11F33 13 F35 (secondary).

Key words and phrases. Eigenvarieties, slopes of $U_{p}$-operator, weight spaces, Newton-Hodge decomposition, overconvergent Hilbert modular forms, completed homology. 
the boundary of the weight space, the eigencurve is a disjoint union of infinitely many connected components that are finite flat over the weight space. We refer to [20, Conjecture 1.2] for the precise statement of the conjecture.

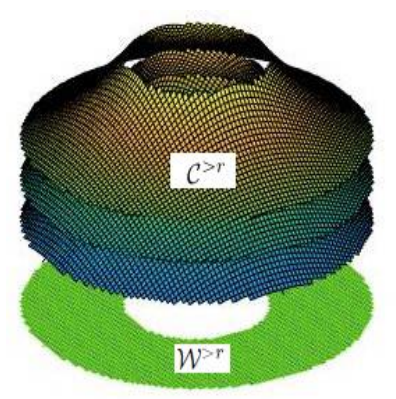

Figure 1. Spectral halo of eigencurves

For eigencurves associated to a definite quaternion algebra $D$ over $\mathbb{Q}$, this conjecture was studied by R. Liu, D. Wan, and L. Xiao in [20]. To be more precise, we fix an odd prime $p$ and denote by $\mathrm{Spc}_{D}$ the spectral curve associated to the overconvergent automorphic forms on $D / \mathbb{Q}$ (with some tame level). It admits a weight map wt : $\operatorname{Spc}_{D} \rightarrow \mathcal{W}$ to the weight space $\mathcal{W}$, where $\mathcal{W}$ is the rigid analytification of the Iwasawa algebra $\mathbb{Z}_{p} \llbracket \mathbb{Z}_{p}^{\times} \rrbracket$ and a slope map $a_{p}: \mathrm{Spc}_{D} \rightarrow \mathbb{G}_{m}$. For $r \in(0,1)$, we use $\mathcal{W}^{>r}$ to denote the subspace of $\mathcal{W}$ where the fixed parameter $T:=[\exp (p)]-1$ satisfies $|T|_{p} \in(r, 1)$ and let $\operatorname{Spc}_{D}^{>r}=\mathrm{wt}^{-1}\left(\mathcal{W}^{>r}\right)$. Under the above notations, Liu-Wan-Xiao proved the following theorem.

Theorem 1.0.1. The space $\mathrm{Spc}_{D}^{>1 / p}$ can be decomposed into a disjoint union

$$
X_{0} \coprod X_{(0,1)} \coprod X_{1} \coprod X_{(1,2)} \coprod X_{2} \coprod \cdots
$$

of rigid analytic spaces which are finite and flat over $\mathcal{W}^{>1 / p}$ via wt such that for each point $x \in X_{I}$ with I denoting the interval $n=[n, n]$ or $(n, n+1)$, we have $v_{p}\left(a_{p}(x)\right) \in$ $(p-1) v_{p}\left(T_{\mathrm{wt}(x)}\right) \cdot I$.

In particular, as $x$ varies on each irreducible component of $\mathrm{Spc}_{D}$ with $\mathrm{wt}(x)$ approaching the boundary of weight space, i.e. $\left|T_{\mathrm{wt}(x)}\right|_{p} \rightarrow 1^{-}$, the slope $v_{p}\left(a_{p}(x)\right) \rightarrow 0$.

By $p$-adic family version of the Jacquet-Langlands correspondence (see [8, Theorem 3]), they also deduce the similar results for most of the components of the original ColemanMazur eigencurves.

The goal of this paper is to generalize the result in [20] to the eigenvarieties associated to ( $p$-adic) overconvergent automorphic forms for a definite quaternion algebra over a totally real field $F$ in which $p$ splits completely. Combining with the $p$-adic family versions of base change and Jacquet-Langlands correspondence, this result allows us to determine the boundary behavior for the entire Coleman-Mazur eigencurves, and hence answers the first part of original question raised by Coleman and Mazur (see [20, Conjecture 1.2(1)]) in complete generality.

We set up a few notations before stating our main result. Let $F$ be a totally real field of degree $g$ and $p$ be an odd prime number which splits completely in $F$. Let $\mathcal{O}_{F}$ be the ring of integers of $F$ and set $\mathcal{O}_{p}:=\mathcal{O}_{F} \otimes_{\mathbb{Z}} \mathbb{Z}_{p}$. Let $I:=\operatorname{Hom}\left(F, \overline{\mathbb{Q}}_{p}\right)$ and for each $i \in I$, we choose a uniformizer $\pi_{i}$ of the completion $F_{\mathfrak{p}_{i}}$ of $F$ at the prime $\mathfrak{p}_{i}$ induced by $i$. The weight space $\mathcal{W}$ is the rigid analytification of $\mathbb{Z}_{p} \llbracket \mathcal{O}_{p}^{\times} \times \mathbb{Z}_{p}^{\times} \rrbracket$. It has a full set of parameters $\left\{\left(T_{i}:=\left[\exp \left(\pi_{i}\right), 1\right]-1\right)_{i \in I}, T:=[1, \exp (p)]-1\right\}$. For $r \in(0,1)$, we use $\mathcal{W}^{>r}$ to denote the subspace of $\mathcal{W}$ where $\left|T_{i}\right|_{p} \in(r, 1)$ for all $i \in I$. Let $D / F$ be a totally definite quaternion 
division algebra over $F$, which is split at all places over $p$. Fix a tame level structure. Let $\mathcal{X}_{D}$ (resp. $\mathcal{Z}_{D}$ ) be the eigenvariety (resp. spectral variety) associated to the overconvergent automorphic forms for $D^{\times}$constructed in [5, Part III]. We put $w: \mathcal{X}_{D} \rightarrow \mathcal{W}$ be the weight map and denote by $\mathcal{X}_{D}^{>r}$ the preimage $w^{-1}\left(\mathcal{W}^{>r}\right)$. For each $x \in \mathcal{X}_{D}\left(\mathbb{C}_{p}\right)$, it corresponds to a $\mathbb{C}_{p}$-valued system of eigenvalues (we refer to $[5, \S 5]$ for the precise definition). For each $i \in I$, there is a Hecke operator $U_{\pi_{i}}$ acting on the space of overconvergent automorphic forms and we use $a_{i}(x)$ to denote the eigenvalue of the $U_{\pi_{i}}$-operator for $x$. The constructions of the eigenvarieties and Hecke operators will be carefully recalled in 3.5 and $\$ 3.6$.

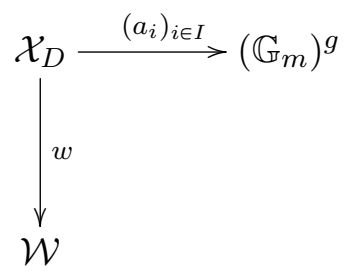

Under the above notations, we have the following theorem.

Theorem 1.0.2. We denote by $\Sigma$ the subset $\{0\} \bigcup\left\{1+2 k \mid k \in \mathbb{Z}_{\geqslant 0}\right\}$ of $\mathbb{Z}$. The eigenvariety $\mathcal{X}_{D}^{>1 / p}$ is a disjoint union

$$
\mathcal{X}_{D}^{>1 / p}=\bigsqcup_{l \in \Sigma^{I}, \sigma \in\{ \pm\}^{I}} \mathcal{X}_{l, \sigma}
$$

of (possibly empty) rigid analytic spaces which are finite over $\mathcal{W}^{>1 / p}$ via $w$, such that for each closed point $x \in \mathcal{X}_{l, \sigma}\left(\mathbb{C}_{p}\right)$ with $l=\left(l_{i}\right)_{i \in I} \in \Sigma^{I}$ and $\sigma=\left(\sigma_{i}\right)_{i \in I} \in\{ \pm\}^{I}$, we have

$$
\begin{cases}v_{p}\left(a_{i}(x)\right)=(p-1) v_{p}\left(T_{i, w(x)}\right) \cdot l_{i}, & \text { for } \sigma_{i}=-, \\ v_{p}\left(a_{i}(x)\right) \in(p-1) v_{p}\left(T_{i, w(x)}\right) \cdot\left(l_{i}, l_{i}+2\right), & \text { for } \sigma_{i}=+ \text { and } l_{i} \neq 0, \\ v_{p}\left(a_{i}(x)\right) \in(p-1) v_{p}\left(T_{i, w(x)}\right) \cdot(0,1), & \text { for } \sigma_{i}=+ \text { and } l_{i}=0,\end{cases}
$$

for all $i \in I$.

In particular, as $x$ varies on each irreducible component of $\mathcal{X}_{D}$ with $w(x)$ approaching the boundary of the weight space, i.e. $v_{p}\left(T_{i, w(x)}\right) \rightarrow 0$, the slopes $v_{p}\left(a_{i}(x)\right) \rightarrow 0$ for all $i \in I$.

Remark 1.0.3. (1) In our main theorem, the decomposition of the eigenvariety $\mathcal{X}_{D}^{>1 / p}$ is characterized by all the $U_{\pi_{i}}$-operators $(i \in I)$. We cannot work solely with the spectral varieties since only the eigenvalues of $U_{\pi}:=\prod_{i \in I} U_{\pi_{i}}$ can be read from the spectral varieties. This is a significant difference to the eigencurve case.

(2) There have been several generalizations of Liu-Wan-Xiao's results to more general eigenvarieties. In [27, Ye generalized Liu-Wan-Xiao's estimation on slopes in eigencurves to eigenvarieties for definite unitary groups of arbitrary rank. Ye gave a lower bound and upper bound of the Newton polygon of characteristic power series for the $U_{p}$-operator, but they do not match at any point on the Newton polygon. So she cannot prove a similar result as in Theorem 1.0.2 for eigenvarieties associated to definite unitary groups.

There are also generalizations to Hilbert modular eigenvarieties. Let $F$ and $p$ be as above. In [17], Johansson and Newton defined a one-dimensional 'partial' eigenvarieties interpolating Hilbert modular forms over $F$ with weight varying only at a single place above $p$. They proved that over the boundary annulus of the weight space, the partial eigenvarieties decompose as a disjoint union of components that are finite over weight space, and the components have a similar property as described in the main result of Liu-Wan-Xiao. Our result agrees with theirs when restricting to their 'partial' eigenvarieties. 
(3) The assumption that $p$ splits completely in $F$ is essential in our argument. In fact, the philosophical analogy between the Artin-Schreier-Witt tower and the Igusa tower of Hilbert modular Shimura varieties explained in [23] and the main theorem there suggests that further modifications are needed in order to formulate a 'reasonable' conjecture that generalizes Theorem 1.0 .2 to general $F$. We refer to 1.3 for more discussion towards the (conjectural) generalization of our theorem.

1.1. Idea of the proof of Theorems 1.0.2. We now explain how we deduce our main result.

I. Going back to Liu-Wan-Xiao's work in [20], the way they deduce their main results over $\mathbb{Q}$ relies crucially on two key ingredients:

(a) a sharp estimate of the action of the $U_{p}$-operator on the space of overconvergent automorphic forms, in the form of providing a lower bound of the Hodge polygon, and

(b) the observation that, at classical weights, the subspaces of classical automorphic forms provide known points of the corresponding Newton polygon which happens to lie on the Hodge polygon.

II. While we choose to work with automorphic forms associated to a definite quaternion algebra over $F$ as opposed to the usual overconvergent modular forms, we circumvent the complication of the geometry of the Hilbert modular Shimura varieties. We will define the spaces of integral $p$-adic automorphic forms. It contains the spaces of overconvergent automorphic forms and have the same characteristic power series of the $U_{\pi}$-operator. An important observation is that the $U_{\pi}$-operator on the spaces of integral $p$-adic automorphic forms can be written reasonably explicitly, as explained in $\$ 4.2$. This was inspired by the thesis 16 of D. Jacobs (a former student of Buzzard), and the generalization in [20].

III. The major difficulty we encounter here is that the action of each individual $U_{\pi_{i}{ }^{-}}$ operator on the space of overconvergent automorphic forms is not compact, whereas the action of their product is. So if we generalize the method in [20] naively, one would have to work with the operator $U_{\pi}=\prod_{i \in I} U_{\pi_{i}}$. It is possible to give a lower bound of the corresponding Hodge polygon similar to $\mathbf{I}(a)$, which is associated to inequalities regarding the sum of all the $U_{\pi_{i}}$-slopes. But for $\mathbf{I}(b)$, the space of classical forms are characterized by the properties of the type: the $U_{\pi_{i}}$-slope is less than or equal to $k_{i}-1$, for every individual $i \in I$. This does not provide a known point on the Newton polygon for the action of $U_{\pi}$, because the ordering mechanism of bases are incompatible. More precisely, there exists no orthonormal basis, in the sense of [10, Definition I.1.3], of the space of continuous function $\mathcal{C}\left(\mathcal{O}_{p}, \mathbb{C}_{p}\right)$, such that the first $d:=\prod_{i \in I}\left(k_{i}-1\right)$ elements of $\Omega$, gives a basis of $\mathcal{C}\left(\mathcal{O}_{p}, \mathbb{C}_{p}\right)^{\operatorname{deg} \leqslant k-2}$ for all $k=\left(k_{i}\right)_{i \in I} \in \mathbb{Z}_{\geqslant 2}^{I}$, where $\mathcal{C}\left(\mathcal{O}_{p}, \mathbb{C}_{p}\right)^{\text {deg } \leqslant k-2}$ is the $\mathbb{C}_{p}$-subspace of $\mathcal{C}\left(\mathcal{O}_{p}, \mathbb{C}_{p}\right)$ spanned by the polynomial functions $\prod_{i \in I} z_{i}^{l_{i}}$, for all $0 \leqslant l_{i} \leqslant k_{i}-2, i \in I$, and $z_{i}: \mathcal{O}_{p}=\prod_{i \in I} \mathcal{O}_{\mathfrak{p}_{i}} \rightarrow \mathbb{C}_{p}$ is the projection to the $i$ th component. Therefore, the lower bound of the Hodge polygon of the $U_{\pi^{-}}$-operator does not touch its actual Newton polygon in general when the degree $g=[F: \mathbb{Q}] \geqslant 2$.

IV. To circumvent this difficulty, we work with the space of generalized integral $p$-adic automorphic forms. In the introduction, we explain our idea in the simplest case when $[F: \mathbb{Q}]=2$. Let $\mathfrak{p}_{1}, \mathfrak{p}_{2}$ be the two places of $F$ over $p$. 
Let $A$ be a topological ring in which $p$ is topologically nilpotent. For a continuous homomorphism $\kappa: \mathcal{O}_{p}^{\times} \times \mathcal{O}_{p}^{\times} \rightarrow A^{\times}$, we define the space of integral $p$-adic automorphic forms $S_{\kappa, I}^{D}\left(K^{p}, A\right)$ and the space of generalized $p$-adic automorphic forms $S_{\kappa, 1}^{D}\left(K^{p}, A\right)$. The latter space consists of generalized automorphic forms that are like automorphic forms at the place $\mathfrak{p}_{1}$, but are like the continuous dual of the completed homology at the place $\mathfrak{p}_{2}$. We have explicit isomorphisms of these spaces:

$$
S_{\kappa, I}^{D}\left(K^{p}, A\right) \cong \bigoplus_{k=0}^{s-1} \mathcal{C}\left(\mathcal{O}_{p}, A\right) \text { and } S_{\kappa, 1}^{D}\left(K^{p}, A\right) \cong \bigoplus_{k=0}^{s-1} \mathcal{C}_{1}(\kappa, A)
$$

where $\mathcal{C}_{1}(\kappa, A)$ is a suitably defined subspace of $\mathcal{C}\left(\mathcal{O}_{\mathfrak{p}_{1}} \times \operatorname{Iw}_{\pi_{2}}, A\right)$ that contains $\mathcal{C}\left(\mathcal{O}_{p}, A\right)$. We use $S_{\kappa, I}^{D}\left(K^{p}, A\right)^{\vee}$ (resp. $\left.S_{\kappa, 1}^{D}\left(K^{p}, A\right)^{\vee}\right)$ to denote the continuous $A$ dual space of $S_{\kappa, I}^{D}\left(K^{p}, A\right)$ (resp. $S_{\kappa, 1}^{D}\left(K^{p}, A\right)$ ). Our argument can be exhibited in the following diagram:

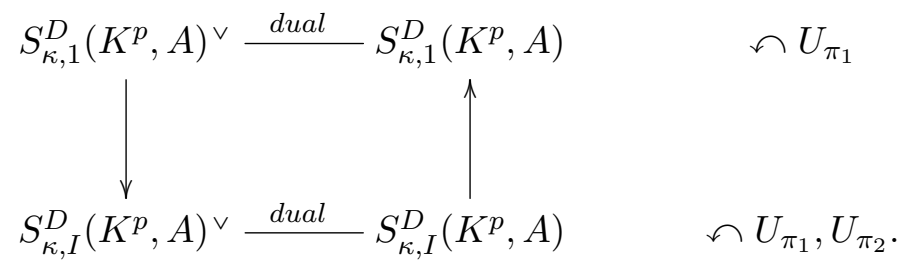

Here the right vertical arrow is an embedding $S_{\kappa, I}^{D}\left(K^{p}, A\right) \hookrightarrow S_{\kappa, 1}^{D}\left(K^{p}, A\right)$, which identifies $S_{\kappa, I}^{D}\left(K^{p}, A\right)$ as the invariant subspace of $S_{\kappa, 1}^{D}\left(K^{p}, A\right)$ under an action of the Borel subgroup $B\left(\mathcal{O}_{\mathfrak{p}_{2}}\right)$ of $\mathrm{GL}_{2}\left(\mathcal{O}_{\mathfrak{p}_{2}}\right)$; and the left vertical arrow is the dual of this embedding, i.e. taking the $B\left(\mathcal{O}_{\mathfrak{p}_{2}}\right)$-coinvariants of $S_{\kappa, 1}^{D}\left(K^{p}, A\right)^{\vee}$.

We first embed $S_{\kappa, I}^{D}\left(K^{p}, A\right)$ into the larger space $S_{\kappa, 1}^{D}\left(K^{p}, A\right)$. The latter space carries an extra structure of right $A \llbracket P_{2}^{\prime} \rrbracket$-modules, where $P_{2}^{\prime} \subset \mathrm{SL}_{2}\left(\mathcal{O}_{\mathfrak{p}_{2}}\right)$ is some explicit open compact pro- $p$-subgroup. The sacrifice of doing this is that there is only $U_{\pi_{1}}$-operator, but no $U_{\pi_{2}}$-operator defined on $S_{\kappa, 1}^{D}\left(K^{p}, A\right)$. Thus the space $S_{\kappa, 1}^{D}\left(K^{p}, A\right)^{\vee}$ is an infinite free left $A \llbracket P_{2}^{\prime} \rrbracket$-module and the induced $U_{\pi_{1}}$-operator on it is $A \llbracket P_{2}^{\prime} \rrbracket$-linear. Under a suitable chosen basis of $S_{\kappa, 1}^{D}\left(K^{p}, A\right)^{\vee}$, we verify that the infinite matrix $M$ corresponding to the $U_{\pi_{1}}$-operator admits a similar estimation as obtained in 20]. On the other hand, we have a characterization of the image of the spaces of classical automorphic forms in $S_{\kappa, 1}^{D}\left(K^{p}, A\right.$ ) (when $A=\mathbb{C}_{p}$ and $\kappa$ is locally algebraic). An analogous argument of $\mathbf{I}(b)$ provides us known points on the Newton polygon of $M$ modulo the augmentation ideal of $A \llbracket P_{2}^{\prime} \rrbracket$. In $\$ 2$, we prove a NewtonHodge decomposition theorem for infinite matrices over certain noncommutative rings, which is a generalization of the Newton-Hodge decomposition theorem over valuation rings. We apply this theorem to the matrix $M$, and obtain a filtration $\left\{\tilde{F}_{\alpha}\right\}$ of the space $S_{\kappa, 1}^{D}\left(K^{p}, A\right)^{\vee}$. Under the surjective map $S_{\kappa, 1}^{D}\left(K^{p}, A\right)^{\vee} \rightarrow S_{\kappa, I}^{D}\left(K^{p}, A\right)^{\vee}$, we obtain a filtration $\left\{F_{\alpha}\right\}$ of $S_{\kappa, I}^{D}\left(K^{p}, A\right)^{\vee}$. We will show that this filtration is stable under the $U_{\pi_{1}}$ and $U_{\pi_{2}}$-operators on $S_{\kappa, I}^{D}\left(K^{p}, A\right)^{\vee}$. When $\kappa: \mathcal{O}_{p}^{\times} \times \mathcal{O}_{p}^{\times} \rightarrow A^{\times}$ is associated to a point $x \in \mathcal{W}^{>1 / p}\left(\mathbb{C}_{p}\right)$, the graded pieces of this filtration are characterized by the condition in Theorem 1.0 .2 for the single place $i=\mathfrak{p}_{1}$. We run the above argument to the graded pieces of the filtration $\left\{F_{\alpha}\right\}$ and the $U_{\pi_{2}}$-operator on it. We get a filtration for every graded piece, and the graded pieces of all these filtrations are characterized by the desired property in Theorem 1.0.2. Our main theorem follows form the existence of such filtrations. 
1.2. Applications. Let $F$ and $p$ be as above and $\mathfrak{n}$ be an ideal of $F$ prime to $p$. We use $\mathcal{X}_{\mathrm{GL}_{2 / F}}(\mathfrak{n})$ to denote the Hilbert modular eigenvariety of tame level $\mathfrak{n}$, which admits a weight map $w: \mathcal{X}_{\mathrm{GL}_{2 / F}}(\mathfrak{n}) \rightarrow \mathcal{W}$. Similar as before, we can define $\mathcal{X}_{\mathrm{GL}_{2 / F}}(\mathfrak{n})^{>1 / p}$. An important consequence of Theorem 1.0 .2 is the following description of the full Hilbert modular eigenvarieties over the boundary of the weight space.

Theorem 1.2.1. The eigenvariety $\mathcal{X}_{\mathrm{GL}_{2 / F}}(\mathfrak{n})^{>1 / p}$ is a disjoint union

$$
\mathcal{X}_{\mathrm{GL}_{2 / F}}(\mathfrak{n})^{>1 / p}=\bigsqcup_{l \in \Sigma^{I}, \sigma \in\{ \pm\}^{I}} \mathcal{X}_{l, \sigma}
$$

of (possibly empty) rigid analytic spaces which are finite over $\mathcal{W}^{>1 / p}$ via $w$, such that for each closed point $x \in \mathcal{X}_{l, \sigma}\left(\mathbb{C}_{p}\right)$ with $l=\left(l_{i}\right)_{i \in I} \in \Sigma^{I}$ and $\sigma=\left(\sigma_{i}\right)_{i \in I} \in\{ \pm\}^{I}$, we have

$$
\begin{cases}v_{p}\left(a_{i}(x)\right)=(p-1) v_{p}\left(T_{i, w(x)}\right) \cdot l_{i}, & \text { for } \sigma_{i}=-, \\ v_{p}\left(a_{i}(x)\right) \in(p-1) v_{p}\left(T_{i, w(x)}\right) \cdot\left(l_{i}, l_{i}+2\right), & \text { for } \sigma_{i}=+ \text { and } l_{i} \neq 0, \\ v_{p}\left(a_{i}(x)\right) \in(p-1) v_{p}\left(T_{i, w(x)}\right) \cdot(0,1), & \text { for } \sigma_{i}=+ \text { and } l_{i}=0,\end{cases}
$$

for all $i \in I$.

This theorem will be proved in \$6. The main tool of our proof is Hansen's $p$-adic interpolation theorem $([12$, Theorem 5.1.6]). When the degree $[F: \mathbb{Q}]$ is even, there is an isomorphism between $\mathcal{X}_{\mathrm{GL}_{2 / F}}(\mathfrak{n})$ and the eigenvariety $\mathcal{X}_{D}(\mathfrak{n})$ for the totally definite quaternion algebra $D$ over $F$ with discriminant 1 . The theorem above follows directly from Theorem 1.0.2. When $[F: \mathbb{Q}]$ is odd, we take a quadratic extension $F^{\prime} / F$ such that $F^{\prime}$ is totally real and $p$ splits completely in $F^{\prime}$. Set $\mathfrak{n}^{\prime}=\mathfrak{n} \mathcal{O}_{F}$. We show that there exists a morphism $\mathcal{X}_{\mathrm{GL}_{2 / F}}(\mathfrak{n}) \rightarrow \mathcal{X}_{\mathrm{GL}_{2 / F^{\prime}}}\left(\mathfrak{n}^{\prime}\right)$ that interpolates the quadratic base change from $F$ to $F^{\prime}$ on non-critical classical points. Then the theorem for $\mathcal{X}_{\mathrm{GL}_{2 / F}}(\mathfrak{n})$ follows from that for $\mathcal{X}_{\mathrm{GL}_{2 / F^{\prime}}}\left(\mathfrak{n}^{\prime}\right)$.

A notable consequence of the above theorem is that every irreducible component of the (full) Hilbert modular eigenvarieties contains a classical point of parallel weight 2. This result has been proven for most irreducible components by Johansson-Newton in [17]. They use this result to prove the parity conjecture for Hilbert modular forms unconditionally when the degree $[F: \mathbb{Q}]$ is even, and when the degree $[F: \mathbb{Q}]$ is odd, they need to impose the assumption that the automorphic representation corresponding to the Hilbert modular form is not principal series at some place prime to $p$. The last assumption is to guarantee that the Hilbert modular form corresponds to an automorphic form for a definite quaternion algebra over $F$ under the Jacquet-Langlands correspondence. This assumption can be removed now.

1.3. Further questions. Theorem 1.0 .2 is not a complete description of the boundary behavior of the eigenvariety $\mathcal{X}_{D}$. Inspired by Coleman-Mazur-Buzzard-Kilford conjecture ( 20 , Conjecture 1.2]), we make the following conjecture.

Conjecture 1.3.1. When $r \in(0,1)$ is sufficiently close to $1^{-}$, there exists a sequence of rational numbers $\Sigma_{i}=\left\{\alpha_{i, 0} \leqslant \alpha_{i, 1} \leqslant \ldots\right\}$ for every $i \in I$, such that if we denote $\Sigma:=\prod_{i \in I} \Sigma_{i}$, then the eigenvariety $\mathcal{X}_{D}^{>r}$ is a disjoint union

$$
\mathcal{X}_{D}^{>r}=\bigsqcup_{\alpha=\left(\alpha_{i}\right)_{i \in I} \in \Sigma} \mathcal{X}_{\alpha}
$$

of (possibly empty) rigid analytic spaces which are finite over $\mathcal{W}^{>r}$ via $w$. For every $\alpha \in \Sigma$ and each closed point $x \in \mathcal{X}_{\alpha}\left(\mathbb{C}_{p}\right)$, we have $v_{p}\left(a_{i}(x)\right)=(p-1) v_{p}\left(T_{i, w(x)}\right) \cdot \alpha_{i}$ for all $i \in I$. 
Moreover, each sequence $\Sigma_{i}$ is a disjoint union of finitely many arithmetic progressions, counted with multiplicities.

When $F=\mathbb{Q}$, Conjecture 1.3.1 has been proved for the eigenvariety $\mathcal{X}_{D}^{>r}$ for some (explicit) rational number $r \in\left(\frac{1}{p}, 1\right)$ in $[20$, Theorem 1.5]. Their proof is based on a careful analysis of the characteristic power series of the $U_{p}$-operator and the fact that the coefficients of the characteristic power series belong to the Iwasawa algebra $\Lambda=\mathbb{Z}_{p} \llbracket \mathbb{Z}_{p}^{\times} \rrbracket$. It seems to us that more ideas are needed in order to generalize their result to the case of Hilbert modular eigenvarieties. In [3, Conjecture 1], Birkbeck made a similar conjecture on the $p$ adic slopes of overconvergent Hilbert modular forms without any assumption on the totally real field $F$. Our Conjecture 1.3.1 makes the assumption that $p$ splits in $F$, and hence gives a more precise characterization of the decomposition of the eigenvarieties. We also refer to Birkbeck's thesis [4, Chapter 8] for numerical evidence towards Conjecture 1.3.1.

Finally, we make a comment about the case for $p=2$. Most of our arguments work for $p=2$ with mild modifications. However, there is one subtle place in Notation 3.7.5 that the oddness of $p$ is crucial. We explain the case for $F=\mathbb{Q}$ as an example and refer to Notation 3.7 .5 for details. Let $\operatorname{Iw}_{p}=\left(\begin{array}{c}\mathbb{Z}_{p}^{\times} \\ \mathbb{Z}_{p} \\ \mathbb{Z}_{p}^{\times}\end{array}\right)$be the Iwahori subgroup of $\mathrm{GL}_{2}\left(\mathbb{Z}_{p}\right)$ and $\left.D\left(\mathbb{Z}_{p}^{\times}\right)=\left\{\begin{array}{cc}\alpha & 0 \\ 0 & \alpha\end{array}\right) \mid \alpha \in \mathbb{Z}_{p}^{\times}\right\}$be the subgroup of $\operatorname{Iw}_{p}$ consisting of scalar matrices. When $p>2$, we show that the inclusion map $D\left(\mathbb{Z}_{p}\right) \hookrightarrow \operatorname{Iw}_{p}$ has a section, i.e. there exists a subgroup $P$ of $\operatorname{Iw}_{p}$ such that the multiplication map $D\left(\mathbb{Z}_{p}\right) \times P \rightarrow \mathrm{Iw}_{p}$ is an isomorphism. We encourage the audience to explore how to modify the argument for $p=2$.

1.4. Structure of the paper. The $\$ 2$ is devoted to prove a Newton-Hodge decomposition theorem for infinite matrices over certain noncommutative rings. This section is technical and the readers can assume the main result Theorem 2.3.13 and skip its lengthy proof at first. In $\$ 3$, we first recall the notions of overconvergent automorphic forms, Hecke operators and eigenvarieties. Then we construct a space of generalized integral $p$-adic automorphic forms and explain its relation with the spaces of classical automorphic forms. In \$4 we give an explicit expression of the Hecke operator $U_{\pi_{i}}$ on the space of generalized integral $p$-adic automorphic forms, and use the Newton-Hodge decomposition Theorem 2.3.13 to obtain a filtration of this space, whose graded piece has a nice description in terms of the $U_{\pi_{i}}$-action, for a fixed $i \in I$. We complete the proof of Theorem 1.0.2 in $\$ 5$, The idea is to inductively apply the argument in the previous section to all places of $F$ over $p$, and then get a filtration of the space of integral $p$-adic automorphic forms, whose graded pieces can be described in terms of all the $U_{\pi_{i}}$-operators. The decomposition of the eigenvarieties follows from the existence of such a filtration. In $\$ 6$, we use Hansen's $p$-adic interpolation theorem to translate our results to Hilbert modular eigenvarieties.

1.5. Acknowledgment. We thank Liang Xiao for sharing his ideas and answering many questions on this topic. We would also like to thank Yiwen Ding, Yongquan Hu and Daqing Wan for helpful comments and conversations. Finally we would like to thank the anonymous referees for their impressively helpful report that significantly improved the exposition of this paper.

1.6. Notations. For every prime $p$, we fix an embedding $\iota_{p}: \overline{\mathbb{Q}} \rightarrow \mathbb{C}_{p}$. We use $v_{p}(\cdot)$ (resp. $|\cdot|_{p}$ ) to denote the $p$-adic valuation (resp. $p$-adic norm) on $\mathbb{C}_{p}$, normalized by $v_{p}(p)=1$ (resp. $|p|_{p}=p^{-1}$ ).

For two topological spaces $X$ and $Y$, we use $\mathcal{C}(X, Y)$ to denote the set of continuous maps from $X$ to $Y$. 


\section{NeWton-Hodge Decomposition Over CERTAin NONCOMMUtATIVE RINGS}

\subsection{Notations.}

- Let $\mathbb{N}=\{1,2, \ldots\}$. For an integer $n>0$, we denote by $[n]:=\{1,2, \ldots, n\} \subset \mathbb{N}$ and $[\infty]:=\mathbb{N} \cup\{\infty\}$.

- For an increasing sequence $\underline{\lambda}=\left(\lambda_{1}, \lambda_{2}, \ldots\right)$ of real numbers, we denote by $\underline{\lambda}^{[n]}:=$ $\left(\lambda_{1}, \ldots, \lambda_{n}\right)$ the $n$-th truncated subsequence of $\underline{\lambda}$. More generally, for two integers $0<m<n$, we put $\underline{\lambda}^{(m, n]}:=\left(\lambda_{m+1}, \ldots, \lambda_{n}\right)$.

- For $m, n \in[\infty]$ and a ring $R$, we denote by $\mathrm{M}_{m \times n}(R)$ the set of $m \times n$ matrices with entries in $R$ and abbreviate it by $\mathrm{M}_{n}(R)$ when $m=n$.

- For any $n \in[\infty], M \in \mathrm{M}_{n}(R)$ and $I, J$ two subsets of $[n]$ with cardinalities $|I|=k$, $|J|=l$, we denote by $M_{I, J}$ the $k \times l$ matrix consisting of entries of $M$ with row indices in $I$ and column indices in $J$, and abbreviate it by $M_{I}$ when $I=J$.

- We denote by $v_{T}(-)$ the $T$-adic valuation of the formal power series ring $\mathbb{F}_{p} \llbracket T \rrbracket$ normalized by $v_{T}(T)=1$.

- We denote by $\mathbf{C}$ an algebraic closure of the fractional field $\mathbb{F}_{p}((T))$ of $\mathbb{F}_{p} \llbracket T \rrbracket$. The valuation $v_{T}(-)$ extends uniquely to $\mathbf{C}$, which is still denoted by $v_{T}(-)$.

2.2. Newton-Hodge decomposition for matrices in $M_{n}\left(\mathbb{F}_{p} \llbracket T \rrbracket\right)$.

Definition 2.2.1. (1) The Newton and Hodge functions of a matrix $M \in M_{n}\left(\mathbb{F}_{p} \llbracket T \rrbracket\right)$ are defined by

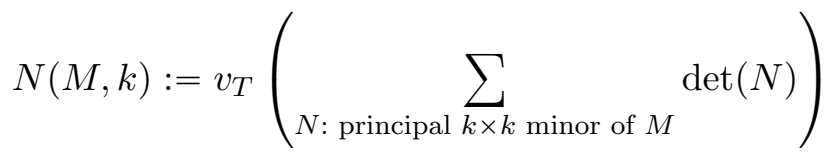

and

$$
H(M, k):=\min \left\{v_{T}(\operatorname{det}(N)) \mid N \text { is a } k \times k \text { minor of } M\right\}
$$

for every $k \in[n]$.

(2) Moreover, we set $H(M, 0):=0$ and $N(M, 0):=0$. The Hodge polygon (resp. Newton polygon) of $M$ is the lower convex hull of the points $\{(k, H(M, k))\}_{k=0}^{n}$ (resp. $\left.\{(k, N(M, k))\}_{k=0}^{n}\right)$.

Remark 2.2.2. Let $\alpha_{1}, \ldots, \alpha_{n}$ be the eigenvalues of $M$ in $\mathbf{C}$ (counted with multiplicities) such that $v_{T}\left(\alpha_{1}\right) \leqslant \cdots \leqslant v_{T}\left(\alpha_{n}\right)$. Then the slopes of the Newton polygon are exactly $v_{T}\left(\alpha_{1}\right), \ldots, v_{T}\left(\alpha_{n}\right)$.

Let $\underline{\lambda}=\left(\lambda_{1}, \lambda_{2}, \ldots\right)$ be a (not necessarily strictly) increasing sequence of nonnegative real numbers such that $\lim _{n \rightarrow+\infty} \lambda_{n}=+\infty$. For every integer $n>0$, we denote by $D\left(\underline{\lambda}^{[n]}\right)$ (resp. $D(\underline{\lambda}))$ the diagonal matrix $\operatorname{Diag}\left(T^{\lambda_{1}}, \ldots, T^{\lambda_{n}}\right) \in \mathrm{M}_{n}\left(\mathbb{F}_{p} \llbracket T \rrbracket\right)\left(\operatorname{resp} . \operatorname{Diag}\left(T^{\lambda_{1}}, \ldots\right) \in\right.$ $\left.\mathrm{M}_{\infty}\left(\mathbb{F}_{p} \llbracket T \rrbracket\right)\right)$. For every two integers $0<m<n$, we denote by $D\left(\underline{\lambda}^{(m, n]}\right)$ the diagonal $\operatorname{matrix} \operatorname{Diag}\left(T^{\lambda_{m+1}}, \ldots, T^{\lambda_{n}}\right) \in \mathrm{M}_{n-m}\left(\mathbb{F}_{p} \llbracket T \rrbracket\right)$.

Definition 2.2.3. For $\ell \in \mathbb{N}$ and $n \in \mathbb{N}$,

(1) a matrix $M=\left(m_{i, j}\right)_{1 \leqslant i \leqslant n, 1 \leqslant j \leqslant \ell} \in \mathrm{M}_{n \times \ell}\left(\mathbb{F}_{p} \llbracket T \rrbracket\right)$ is called $\underline{\lambda}^{[n]}$-Hodge bounded if $M=D\left(\underline{\lambda}^{[n]}\right) M^{\prime}$ for some $M^{\prime} \in \mathrm{M}_{n \times \ell}\left(\mathbb{F}_{p} \llbracket T \rrbracket\right)$, or equivalently, $v_{T}\left(m_{i j}\right) \geqslant \lambda_{i}$ for all $1 \leqslant i \leqslant n$ and $1 \leqslant j \leqslant \ell$. When $\ell=n$ and $M^{\prime} \in \mathrm{GL}_{n}\left(\mathbb{F}_{p} \llbracket T \rrbracket\right)$, we call $M$ as strictly $\underline{\lambda}^{[n]}$-Hodge bounded.

(2) A matrix $M \in \mathrm{M}_{\infty}\left(\mathbb{F}_{p} \llbracket T \rrbracket\right)$ is called $\underline{\lambda}$-Hodge bounded if $M=D(\underline{\lambda}) M^{\prime}$ for some $M^{\prime} \in \mathrm{M}_{\infty}\left(\mathbb{F}_{p} \llbracket T \rrbracket\right)$. 
(3) A matrix $A \in \mathrm{M}_{n}\left(\mathbb{F}_{p} \llbracket T \rrbracket\right)$ is called $\underline{\lambda}^{[n]}$-stable if for every $\underline{\lambda}^{[n]}$-Hodge bounded matrix $B \in \mathrm{M}_{n}\left(\mathbb{F}_{p} \llbracket T \rrbracket\right), A B$ is also $\underline{\underline{\lambda}}^{[n]}$-Hodge bounded.

(4) A matrix $A \in \mathrm{M}_{\infty}\left(\mathbb{F}_{p} \llbracket T \rrbracket\right)$ is called $\underline{\lambda}$-stable if for every $\underline{\lambda}$-Hodge bounded matrix $B \in \mathrm{M}_{\infty}\left(\mathbb{F}_{p} \llbracket T \rrbracket\right), A B$ is also $\underline{\lambda}$-Hodge bounded.

Remark 2.2.4. (1) For $A, B \in \mathrm{M}_{n}\left(\mathbb{F}_{p} \llbracket T \rrbracket\right)$ if $B$ is $\underline{\lambda}^{[n]}$-Hodge bounded, then $B A$ is also $\underline{\lambda}^{[n]}$-Hodge bounded.

(2) Let $M \in \mathrm{M}_{\infty}\left(\mathbb{F}_{p} \llbracket T \rrbracket\right)$ be a $\underline{\lambda}$-Hodge bounded matrix. Since $\lambda_{i} \stackrel{i \rightarrow \infty}{\longrightarrow} \infty$, the functions (2.1) and (2.2) are well-defined on $M$, which are called the Newton and Hodge functions for $M$, respectively. Moreover, we define the Newton and Hodge polygons for $M$ in a similar way to the ones for matrices of finite dimensions.

Lemma 2.2.5. For $n \in[\infty]$ and a matrix $A=\left(a_{i j}\right) \in \mathrm{M}_{n}\left(\mathbb{F}_{p} \llbracket T \rrbracket\right)$, the following statements are equivalent:

(1) $A$ is $\underline{\lambda}^{[n]}$-stable;

(2) $D\left(\underline{\lambda}^{[n]}\right)^{-1} A D\left(\underline{\lambda}^{[n]}\right) \in \mathrm{M}_{n}(\mathbb{F} p \llbracket T \rrbracket)$;

(3) $v_{T}\left(a_{i j}\right) \geqslant \lambda_{i}-\lambda_{j}$ for every $1 \leqslant i \leqslant n$ and $1 \leqslant j \leqslant n$.

Proof. (1) $\Rightarrow(2)$ Since $A$ is $\underline{\lambda}^{[n]}$-stable and $D\left(\underline{\lambda}^{[n]}\right)$ is $\underline{\lambda}^{[n]}$-Hodge bounded, $A D\left(\underline{\lambda}^{[n]}\right)$ is also $\underline{\underline{\lambda}}^{[n]}$-Hodge bounded, and hence $A D\left(\underline{\lambda}^{[n]}\right)=D\left(\underline{\lambda}^{[n]}\right) B$ for some $B \in \mathrm{M}_{n}(\mathbb{F} p \llbracket T \rrbracket)$. This proves $D\left(\underline{\lambda}^{[n]}\right)^{-1} A D\left(\underline{\lambda}^{[n]}\right) \in \mathrm{M}_{n}\left(\mathbb{F}_{p} \llbracket T \rrbracket\right)$.

$(2) \Rightarrow(3)$ Suppose that we have $A D\left(\underline{\lambda}^{[n]}\right)=D\left(\underline{\lambda}^{[n]}\right) B$ for some $B=\left(b_{i j}\right) \in \mathrm{M}_{n}\left(\mathbb{F}_{p} \llbracket T \rrbracket\right)$. Comparing the $(i, j)$-entries of the matrices on the two sides of this equality, we have $T^{\lambda_{j}} a_{i j}=T^{\lambda_{i}} b_{i j}$, and hence

$$
v_{T}\left(a_{i j}\right)=v_{T}\left(b_{i j}\right)+\lambda_{i}-\lambda_{j} \geqslant \lambda_{i}-\lambda_{j}
$$

$(3) \Rightarrow(1)$ For every $\underline{\lambda}^{[n]}$-Hodge bounded matrix $M=\left(m_{i j}\right) \in \mathrm{M}_{n}\left(\mathbb{F}_{p} \llbracket T \rrbracket\right)$, we have $v_{T}\left(m_{i j}\right) \geqslant \lambda_{i}$ for all $1 \leqslant i \leqslant n$ and $1 \leqslant j \leqslant n$. Then the $(i, j)$-entry of $A M$ is $\sum_{k=1}^{n} a_{i k} m_{k j}$. Since $v_{T}\left(a_{i k}\right) \geqslant \lambda_{i}-\lambda_{k}$, we have $v_{T}\left(a_{i k} m_{k j}\right) \geqslant \lambda_{i}$, and hence $v_{T}\left(\sum_{k=1}^{n} a_{i k} m_{k j}\right) \geqslant \lambda_{i}$. Therefore, we conclude that $A M$ is $\underline{\lambda}^{[n]}$-Hodge bounded and $A$ is $\underline{\lambda}^{[n]}$-stable.

Lemma 2.2.6. Let $M \in \mathrm{M}_{n}\left(\mathbb{F}_{p} \llbracket T \rrbracket\right)$ be strictly $\underline{\lambda}^{[n]}$-Hodge bounded. For a matrix $A \in$ $\mathrm{M}_{n}\left(\mathbb{F}_{p} \llbracket T \rrbracket\right)$ if $A M$ is $\underline{\lambda}^{[n]}-$ Hodge bounded, then $A$ is $\underline{\underline{\lambda}}^{[n]}$-stable.

Proof. Since $M$ is strictly $\underline{\lambda}^{[n]}$-Hodge bounded and $A M$ is $\underline{\lambda}^{[n]}$-Hodge bounded, there are matrices $M^{\prime} \in \mathrm{GL}_{n}\left(\mathbb{F}_{p} \llbracket T \rrbracket\right)$ and $B \in \mathrm{M}_{n}\left(\mathbb{F}_{p} \llbracket T \rrbracket\right)$ such that $M=D\left(\underline{\lambda}^{[n]}\right) M^{\prime}$ and $A M=$ $D\left(\underline{\lambda}^{[n]}\right) B$. Therefore, we have $A=D\left(\underline{\lambda}^{[n]}\right) B M^{\prime-1} D\left(\underline{\lambda}^{[n]}\right)^{-1}$. Combined with Lemma 2.2.5. this equality implies that $A$ is $\underline{\lambda}^{[n]}$-stable.

Lemma 2.2.7. For $n \in \mathbb{N}$ and $M \in M_{n}\left(\mathbb{F}_{p} \llbracket T \rrbracket\right)$ if $M$ is $\underline{\lambda}^{[n]}$-Hodge bounded and satisfies $N(M, n)=\sum_{i=1}^{n} \lambda_{i}$, then $H(M, k)=\sum_{i=1}^{k} \lambda_{i}$ for every $1 \leqslant k \leqslant n$, i.e. the slopes of the Hodge polygon of $M$ are $\lambda_{1}, \lambda_{2}, \ldots, \lambda_{n}$. In particular, $M$ is strictly $\underline{\lambda}^{[n]}$-Hodge bounded. 
Proof. Since $M$ is $\underline{\lambda}^{[n]}$-Hodge bounded, for every $1 \leqslant k \leqslant n$ we have $H(M, k) \geqslant \sum_{i=1}^{k} \lambda_{i}$. Suppose $H(M, k)>\sum_{i=1}^{k} \lambda_{i}$ for some $k$. Let $I:=[k]$. By Laplace expansion, we have

$$
\operatorname{det}(M)=\sum_{J \subset[n],|J|=k} \operatorname{sgn}(I, J) \operatorname{det}\left(M_{I, J}\right) \operatorname{det}\left(M_{I, J}^{c o m p}\right),
$$

where $\operatorname{sgn}(I, J)=(-1)^{\sum_{i \in I} i+\sum_{j \in J} j}$ is the signature of the permutation determined by $I$ and $J$, and $M_{I, J}^{c o m p}$ is the complement $(n-k) \times(n-k)$ minor of $M_{I, J}$.

Since $M$ is $\underline{\lambda}^{[n]}$-Hodge bounded, we have

$$
v_{T}\left(\operatorname{det}\left(M_{I, J}\right)\right) \geqslant H(M, k)>\sum_{i=1}^{k} \lambda_{i} \text { and } v_{T}\left(\operatorname{det}\left(M_{I, J}^{c o m p}\right)\right) \geqslant \sum_{i=k+1}^{n} \lambda_{i},
$$

which together imply $N(M, n)=v_{T}(\operatorname{det}(M))>\sum_{i=1}^{n} \lambda_{i}$, a contradiction. Therefore we have $H(M, k)=\sum_{i=1}^{k} \lambda_{i}$, for all $1 \leqslant k \leqslant n$.

Since $M$ is $\underline{\lambda}^{[n]}$-Hodge bounded, $M=D\left(\underline{\lambda}^{[n]}\right) M^{\prime}$ for some $M^{\prime} \in \mathrm{M}_{n}(\mathbb{F} p \llbracket T \rrbracket)$. Taking determinants and then $T$-adic valuations on both sides of this equality, we have $v_{T}\left(\operatorname{det}\left(M^{\prime}\right)\right)=$ 0 , and hence $M^{\prime} \in \mathrm{GL}_{n}\left(\mathbb{F}_{p} \llbracket T \rrbracket\right)$. So $M$ is strictly $\underline{\lambda}^{[n]}$-Hodge bounded.

Corollary 2.2.8. Let $M \in M_{\infty}\left(\mathbb{F}_{p} \llbracket T \rrbracket\right)$ be $\underline{\lambda}$-Hodge bounded. If there exists an strictly increasing infinite sequence $\underline{s}=\left(s_{1}, s_{2}, \ldots\right)$ of positive integers such that $N\left(M, s_{n}\right)=$ $\sum_{i=1}^{s_{n}} \lambda_{i}$ for every $n \geqslant 1$, then for every $k \geqslant 1$ we have $H(M, k)=\sum_{i=1}^{k} \lambda_{i}$.

Proof. Since $M$ is $\underline{\lambda}$-Hodge bounded, for every $k \geqslant 1$ we have $H(M, k) \geqslant \sum_{i=1}^{k} \lambda_{i}$. For a fixed integer $k \geqslant 1$, we can choose $n \geqslant 1$ such that $s_{n}>k$. From our hypotheses that $N\left(M, s_{n}\right)=\sum_{i=1}^{s_{n}} \lambda_{i}$ and that $M$ is $\underline{\lambda}$-Hodge bounded, there exists an $s_{n} \times s_{n}$ principal minor $M_{1}$ of $M$ such that

$$
v_{T}\left(\operatorname{det}\left(M_{1}\right)\right)=\sum_{i=1}^{s_{n}} \lambda_{i}
$$

Since $\underline{\lambda}$ is increasing, $M_{1}$ is $\underline{\lambda}^{\left[s_{n}\right]}$-Hodge bounded. Combined with 2.3 and Lemma 2.2.7 for $M_{1}$, this implies $H\left(M_{1}, k\right)=\sum_{i=1}^{k} \lambda_{i}$. Note that every $k \times k$ minor of $M_{1}$ is also a $k \times k$ minor of $M$. We have $H(M, k) \leqslant H\left(M_{1}, k\right)=\sum_{i=1}^{k} \lambda_{i}$, and hence $H(M, k)=\sum_{i=1}^{k} \lambda_{i}$. Since we choose $k$ arbitrarily, the last equality completes the proof.

Definition 2.2.9. For every $n \in[\infty]$ and $M \in \mathrm{M}_{n}\left(\mathbb{F}_{p} \llbracket T \rrbracket\right)$ we call an integer $k$ a touching vertex of $M$ if it satisfies

- $(k, N(M, k))$ is a vertex of the Newton polygon of $M$, and

- $N(M, k)=H(M, k)$. 
Theorem 2.2.10 ( $\llbracket 19]$ Theorem 4.3.11). For every $n \in \mathbb{N}$ and $M \in \mathrm{M}_{n}\left(\mathbb{F}_{p} \llbracket T \rrbracket\right)$ if $1 \leqslant k<n$ is a touching vertex of $M$, then there exists an invertible matrix $P \in \mathrm{GL}_{n}\left(\mathbb{F}_{p} \llbracket T \rrbracket\right)$ such that

$$
P M P^{-1}=k\left[\begin{array}{c:c} 
& k \\
M_{11} & M_{12} \\
\hdashline 0 & M_{22}
\end{array}\right]
$$

where $M_{11}$ and $M_{22}$ are $k \times k$ and $(n-k) \times(n-k)$ matrices such that the matrix $M_{11}$ accounts for the first $k$ slopes of the Hodge and Newton polygon of $M$, while $M_{22}$ accounts for the others.

Proof. See [19, Theorem 4.3.11].

Lemma 2.2.11. For every $n \in \mathbb{N}$ and $M \in \mathrm{M}_{n}\left(\mathbb{F}_{p} \llbracket T \rrbracket\right)$ if $\mu_{1} \leqslant \mu_{2} \leqslant \cdots \leqslant \mu_{n}$ are the slopes of the Hodge polygon of some matrix, then there exists an invertible matrix $P \in \mathrm{GL}_{n}\left(\mathbb{F}_{p} \llbracket T \rrbracket\right)$ such that $P M P^{-1}$ is $\underline{\mu}$-Hodge bounded, where $\underline{\mu}=\left(\mu_{1}, \mu_{2}, \ldots, \mu_{n}\right)$.

Proof. By [19, Theorem 4.3.4], there exist matrices $U, V \in \mathrm{GL}_{n}\left(\mathbb{F}_{p} \llbracket T \rrbracket\right)$ such that $U M V=$ $D(\underline{\mu})$. Right-multiplying $V^{-1} U^{-1}$ to both sides of this equality, we prove that $U M U^{-1}=$ $D(\underline{\mu}) V^{-1} U^{-1}$ is $\underline{\mu}$-Hodge bounded.

Theorem 2.2.12. Let $M \in M_{n}\left(\mathbb{F}_{p} \llbracket T \rrbracket\right)$ be a $\underline{\lambda}^{[n]}$-Hodge bounded matrix. Assume that $1<s<n$ is a touching vertex of $M$ and $H(M, n)=\sum_{i=1}^{n} \lambda_{i}$. Then there exists a matrix $W \in \mathrm{GL}_{n}\left(\mathbb{F}_{p} \llbracket T \rrbracket\right)$ such that $W M W^{-1}$ is $\underline{\lambda}^{[n]}$-Hodge bounded and a block upper triangular matrix of the form

$$
W M W^{-1}=s\left[\begin{array}{c:c}
s \\
M_{11} & M_{12} \\
\hdashline 0 & M_{22}
\end{array}\right] \text {. }
$$

Moreover, $W$ is $\underline{\underline{\lambda}}^{[n]}$-stable.

Proof. By Lemma 2.2.7, for every $1 \leqslant k \leqslant n$ we have $H(M, k)=\sum_{i=1}^{k} \lambda_{i}$. By Theorem 2.2.10. there exists a matrix $P_{1} \in \mathrm{GL}_{n}\left(\mathbb{F}_{p} \llbracket T \rrbracket\right)$ such that

$$
P_{1} M P_{1}^{-1}=s\left[\begin{array}{c:c}
M_{11}^{\prime} & M_{12}^{\prime} \\
\hdashline 0 & M_{22}^{\prime}
\end{array}\right]
$$

and $M_{11}^{\prime}$ accounts for the first $s$-th slopes of the Newton and Hodge polygons of $M$, while $M_{22}^{\prime}$ accounts for the others.

By Lemma 2.2.11, there exist matrices $Q_{1} \in \mathrm{GL}_{s}\left(\mathbb{F}_{p} \llbracket T \rrbracket\right)$ and $Q_{2} \in \mathrm{GL}_{n-s}\left(\mathbb{F}_{p} \llbracket T \rrbracket\right)$ such that $M_{11}=Q_{1} M_{11}^{\prime} Q_{1}^{-1}$ and $M_{22}=Q_{2} M_{22}^{\prime} Q_{2}^{-1}$ are $\underline{\lambda}^{[s]}$ and $\underline{\lambda}^{(s, n]}$-Hodge bounded, respectively. Set

$$
P_{2}:=\left[\begin{array}{cc}
Q_{1} & 0 \\
0 & Q_{2}
\end{array}\right] \in \mathrm{GL}_{n}\left(\mathbb{F}_{p} \llbracket T \rrbracket\right) \text { and } N:=P_{2}\left(P_{1} M P_{1}^{-1}\right) P_{2}^{-1}=s\left[\begin{array}{c:c}
s \\
M_{11} & M_{12} \\
0 & M_{22}
\end{array}\right],
$$

where $M_{12}=Q_{1} M_{12}^{\prime} Q_{2}^{-1}$.

Let $v_{1}, \ldots, v_{s}$ be the column vectors of $M_{11}$ and $w_{1}, \ldots, w_{n-s}$ be the column vectors of $M_{12}$. From $\operatorname{det}\left(M_{11}\right) \neq 0$ we know that $\left\{v_{1}, \ldots, v_{s}\right\}$ forms a basis of the vector space 
$\left(\mathbb{F}_{p}((T))\right)^{s}$. Hence, for each integer $1 \leqslant k \leqslant n-s$ there exist a set of scalars $\left\{a_{1}, \ldots, a_{s}\right\}$ in $\mathbb{F}_{p}((T))$ such that $w_{k}=\sum_{j=1}^{s} a_{j} v_{j}$. By Cramer's rule, we have $a_{j}=\frac{\operatorname{det}\left(M_{11, k}\right)}{\operatorname{det}\left(M_{11}\right)}$, where $M_{11, k}$ is the $s \times s$ matrix obtained by replacing the $k$-th column vector of $M_{11}$ by $w_{k}$. Since $M_{11, k}$ is an $s \times s$ minor of $N$, by definition of Hodge functions, we have $v_{T}\left(\operatorname{det}\left(M_{11, k}\right)\right) \geqslant v_{T}\left(\operatorname{det}\left(M_{11}\right)\right)$, and hence $v_{T}\left(a_{j}\right) \geqslant 0$ for every $j=1, \ldots, s$, which implies that $M_{12}$ is $\underline{\lambda}^{[s]}$-Hodge bounded. Let $W=P_{2} P_{1} \in \mathrm{GL}_{n}\left(\mathbb{F}_{p} \llbracket T \rrbracket\right)$. From the above computation, we obtain that $W M W^{-1}$ is $\underline{\lambda}^{[n]}$-Hodge bounded, and hence so is $W M$. Combined with Lemma 2.2.6 and that $M$ is strictly $\underline{\lambda}^{[n]}$-Hodge bounded, this implies that $W$ is $\underline{\lambda}^{[n]}$-stable.

Lemma 2.2.13. Fix an integer $n \geqslant 1$. Let $A \in M_{n}\left(\mathbb{F}_{p} \llbracket T \rrbracket\right)$ and $\underline{\alpha}:=\left\{\alpha_{1}, \ldots, \alpha_{n}\right\}$ be the set of eigenvalues of $A$ in $\mathbf{C}$. Then there exists $b \in \mathbb{R}$ such that

$$
H\left(A^{k}, 1\right) \geqslant k s+b \text { and } H\left(A^{-k}, 1\right) \geqslant-k S+b
$$

for every $k \geqslant 1$, where

$$
S:=\max _{1 \leqslant i \leqslant n}\left\{v_{T}\left(\alpha_{i}\right)\right\} \text { and } s:=\min _{1 \leqslant i \leqslant n}\left\{v_{T}\left(\alpha_{i}\right)\right\} .
$$

Proof. There exists a matrix $P \in \mathrm{GL}_{n}(\mathbf{C})$ such that $J=P A P^{-1}$ is in Jordan canonical form with diagonal entries $\alpha_{1}, \ldots, \alpha_{n}$. Let $D \in \mathrm{M}_{n}(\mathbf{C})$ be the diagonal matrix with $\alpha_{1}, \ldots, \alpha_{n}$ on the diagonal and set $N:=J-D$. Note that $N$ is nilpotent and commutes with $D$. Let $t:=\min \left\{m \mid N^{m}=0\right\}$. From the decomposition

$$
(D+N)^{k}=\sum_{i=0}^{k}\left(\begin{array}{l}
k \\
i
\end{array}\right) D^{i} N^{k-i}
$$

we have the following estimations

$$
H\left(J^{k}, 1\right) \geqslant \begin{cases}s k & \text { if } H(N, 1) \geqslant s, \\ s(k-t+1)+(t-1) H(N, 1) & \text { if } H(N, 1)<s .\end{cases}
$$

Hence there exists $b_{1} \in \mathbb{R}$ such that $H\left(J^{k}, 1\right) \geqslant k s+b_{1}$ for every $k \geqslant 1$. Similarly, there exists $b_{2} \in \mathbb{R}$ such that $H\left(J^{-k}, 1\right) \geqslant-k S+b_{2}$ for every $k \geqslant 1$.

From $A^{k}=P^{-1} J^{k} P$ and $A^{-k}=P^{-1} J^{-k} P$, we have

$$
H\left(A^{k}, 1\right) \geqslant H\left(P^{-1}, 1\right)+H(P, 1)+H\left(J^{k}, 1\right) \geqslant H\left(P^{-1}, 1\right)+H(P, 1)+b_{1}+k s,
$$

and

$$
H\left(A^{-k}, 1\right) \geqslant H\left(P^{-1}, 1\right)+H(P, 1)+H\left(J^{-k}, 1\right) \geqslant H\left(P^{-1}, 1\right)+H(P, 1)+b_{2}-k S .
$$

Setting $b:=\min \left(b_{1}, b_{2}\right)+H\left(P^{-1}, 1\right)+H(P, 1)$, we complete the proof.

Lemma 2.2.14. Let $1 \leqslant s<n$ be two integers and

$$
M=s\left[\begin{array}{c:c}
A & \vdots \\
\hdashline & B \\
\hdashline & D
\end{array}\right] \in M_{n}\left(\mathbb{F}_{p} \llbracket T \rrbracket\right)
$$

be a $\underline{\lambda}^{[n]}$-Hodge bounded matrix. Assume that

(1) $s$ is a touching vertex of $M$;

(2) $H(C, 1)-\lambda_{s}>0$;

(3) $A$ is strictly $\underline{\lambda}^{[s]}$-Hodge bounded.

Then we have

$$
\max \left\{v_{T}(\alpha) \mid \alpha \text { is an eigenvalue of } A\right\}<\min \left\{v_{T}(\beta) \mid \beta \text { is an eigenvalue of } D\right\} .
$$




\section{Proof. Write}

$a:=\max \left\{v_{T}(\alpha) \mid \alpha\right.$ is an eigenvalue of $\left.A\right\}$ and $b:=\min \left\{v_{T}(\beta) \mid \beta\right.$ is an eigenvalue of $\left.D\right\}$.

From Remark 2.2.2, $a$ (resp. $b$ ) is the largest slope (resp. smallest slope) of the Newton polygon of the matrix $A$ (resp. $D$ ). Since Newton polygon always lies on or above Hodge polygon, we have $a \leqslant \lambda_{s}$ and $b \geqslant \lambda_{s+1} \geqslant \lambda_{s}$.

Set

$$
M_{0}:=s\left[\begin{array}{c:c}
A & B \\
0 & D
\end{array}\right] \in \mathrm{M}_{n}\left(\mathbb{F}_{p} \llbracket T \rrbracket\right) .
$$

Suppose that (2.4) fails. Then we have $a=b=\lambda_{s}$. Denote by $k_{1}$ and $k_{2}$ with $k_{1}<k_{2}$ the $x$-coordinates of two endpoints of the line segment with the slope $\lambda_{s}$ on the Newton polygon of $M_{0}$. Then we have $k_{1}<s<k_{2}, \lambda_{i}=\lambda_{s}$ for $k_{1}+1 \leqslant i \leqslant k_{2}$ and

$$
N\left(M_{0}, k_{t}\right)=H\left(M, k_{t}\right)=\sum_{i=1}^{k_{t}} \lambda_{i} \text { for } t=1,2
$$

where the second equality follows from the hypothesis (2). Now we prove

$$
N\left(M, k_{t}\right)=N\left(M_{0}, k_{t}\right) \text { for } t=1,2 .
$$

For an integer $m \in[n]$, we denote by $F(M, m)$ the sum of all the principal $m \times m$ minors of $M$ and define $F\left(M_{0}, m\right)$ in the same way. We write $M=\left(m_{i, j}\right)_{1 \leqslant i, j \leqslant n}$ and have

$$
F\left(M, k_{t}\right)=\sum_{\substack{I \subset[n] \\|I|=k_{t}}} \sum_{\sigma \in S_{k_{t}}} \operatorname{Sgn}(\sigma) \prod_{i \in I} m_{i, \sigma(i)} .
$$

Note that we have a similar description for $F\left(M_{0}, k_{t}\right)$. From our construction of $M_{0}$, the difference $F\left(M, k_{t}\right)-F\left(M_{0}, k_{t}\right)$ is the sum of terms $\operatorname{Sgn}(\sigma) \prod_{i \in I} m_{i, \sigma(i)}$ such that at least one of the entries $\left\{m_{i, \sigma(i)} \mid i \in I\right\}$ belonging to the block $C$. Combined with the hypothesis (2) and that $M$ is $\underline{\lambda}^{[n]}$-Hodge bounded, this implies

$$
v_{T}\left(F\left(M, k_{t}\right)-F\left(M_{0}, k_{t}\right)\right)>\sum_{i=1}^{k_{t}} \lambda_{i}=H\left(M, k_{t}\right) .
$$

Together with (2.5), this inequality proves (2.6).

Therefore, the point $(s, N(M, s))$ lies on the line segment with endpoints $\left(k_{t}, N\left(M_{0}, k_{t}\right)\right)$ for $t=1,2$, and hence cannot be a vertex of the Newton polygon of $M$, which is a contradiction to our hypothesis (1).

Lemma 2.2.15. For every integer $n \geqslant 1$ and $A \in M_{n}\left(\mathbb{F}_{p} \llbracket T \rrbracket\right)$ if $A$ is strictly $\underline{\lambda}^{[n]}$-Hodge bounded, then for every integer $m \geqslant 1$ we have $H\left(A^{-m}, 1\right) \geqslant-m \lambda_{n}$.

Proof. We denote by $A^{a d}=\left(a_{k, l}^{\prime}\right) \in M_{n}\left(\mathbb{F}_{p} \llbracket T \rrbracket\right)$ the adjoint matrix of $A$, i.e. $a_{k, l}^{\prime}=$ $(-1)^{k+l} \operatorname{det}\left(A_{k, l}\right)$, where $A_{k, l}$ is the $(n-1) \times(n-1)$ minor of $A$ by removing the $k$-th row and $l$-th column. So we have $H\left(A^{a d}, 1\right) \geqslant H(A, n-1)$ and hence for every $m \geqslant 1$,

$$
H\left(\left(A^{a d}\right)^{m}, 1\right) \geqslant m H\left(A^{a d}, 1\right) \geqslant m H(A, n-1)=m \sum_{i=1}^{n-1} \lambda_{i} .
$$

Combined with $A^{-1}=\operatorname{det}(A)^{-1} A^{a d}$, this inequality implies

$$
H\left(A^{-m}, 1\right)=H\left(\left(A^{a d}\right)^{m}, 1\right)-m v_{T}(\operatorname{det}(A)) \geqslant m \sum_{i=1}^{n-1} \lambda_{i}-m \sum_{i=1}^{n} \lambda_{i}=-m \lambda_{n}
$$


Lemma 2.2.16. For every $n \in \mathbb{N}$ if $A \in \mathrm{GL}_{n}\left(\mathbb{F}_{p} \llbracket T \rrbracket\right)$ and $B \in M_{n}\left(\mathbb{F}_{p} \llbracket T \rrbracket\right)$ such that $H(B, 1)>0$, then $A+B \in \mathrm{GL}_{n}\left(\mathbb{F}_{p} \llbracket T \rrbracket\right)$.

Proof. Let

$$
C:=\left(\sum_{i=0}^{\infty}\left(-A^{-1} B\right)^{i}\right) A^{-1}
$$

From the hypothesis $H(B, 1)>0$, we know that $C$ is well-defined and

$$
(A+B) C=C(A+B)=I_{n} .
$$

Lemma 2.2.17. Let $1 \leqslant s<n$ be two integers and

$$
M=s\left[\begin{array}{c:c}
s & \\
A & B \\
C & D
\end{array}\right] \in M_{n}\left(\mathbb{F}_{p} \llbracket T \rrbracket\right) .
$$

be a $\underline{\lambda}^{[n]}$-Hodge bounded matrix.

Assume that $M$ satisfies all the three hypotheses in Lemma 2.2.14. Then there exists a matrix $X \in \mathrm{M}_{(n-s) \times s}\left(\mathbb{F}_{p} \llbracket T \rrbracket\right)$ with $H(X, 1) \geqslant H(C, 1)-\lambda_{s}$ such that

$$
\left[\begin{array}{cc}
I_{s} & 0 \\
X & I_{n-s}
\end{array}\right]\left[\begin{array}{cc}
A & B \\
C & D
\end{array}\right]\left[\begin{array}{cc}
I_{s} & 0 \\
-X & I_{n-s}
\end{array}\right]=s\left[\begin{array}{c:c}
A^{\prime} & B^{\prime} \\
\hdashline 0 & D^{\prime}
\end{array}\right]
$$

and $\left[\begin{array}{cc}I_{s} & 0 \\ X & I_{n-s}\end{array}\right]$ is $\underline{\lambda}^{[n]}$-stable.

Proof. We first prove the following claim.

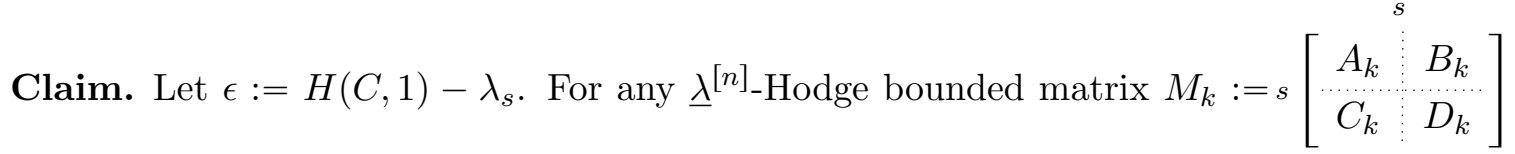
that satisfies all three hypotheses in Lemma 2.2.14 and $H\left(C_{k}, 1\right)-\lambda_{s} \geqslant k \epsilon$ for some integer $k>0$, there exists a matrix $X_{k} \in \mathrm{M}_{(n-s) \times s}\left(\mathbb{F}_{p} \llbracket T \rrbracket\right)$ such that if we set

$$
M_{k+1}:=s\left[\begin{array}{c:c}
A_{k+1} & B_{k+1} \\
C_{k+1} & D_{k+1}
\end{array}\right]=\left[\begin{array}{cc}
I_{s} & 0 \\
X_{k} & I_{n-s}
\end{array}\right] M_{k}\left[\begin{array}{cc}
I_{s} & 0 \\
-X_{k} & I_{n-s}
\end{array}\right]
$$

then

(i) $H\left(X_{k}, 1\right) \geqslant k \epsilon$;

(ii) $H\left(C_{k+1}, 1\right)-\lambda_{s} \geqslant(k+1) \epsilon$;

(iii) the matrix $\left[\begin{array}{cc}I_{s} & 0 \\ X_{k} & I_{n-s}\end{array}\right]$ is $\underline{\lambda}^{[n]}$-stable;

(iv) the matrix $M_{k+1}$ also satisfies all the three hypotheses in Lemma 2.2.14

We now prove that $X_{k}:=-\sum_{i=0}^{\infty} D_{k}^{i} C_{k} A_{k}^{-i-1}$ satisfies all desired properties. We first check that $X_{k}$ is well-defined. Let

$S_{A_{k}}:=\max \left\{v_{T}(\alpha) \mid \alpha\right.$ is an eigenvalue of $\left.A_{k}\right\}$ and $s_{D_{k}}:=\min \left\{v_{T}(\beta) \mid \beta\right.$ is an eigenvalue of $\left.D_{k}\right\}$.

By Lemmas 2.2.13 and 2.2.14, we have $S_{A_{k}}<s_{D_{k}}$ and that there exists $b_{k} \in \mathbb{R}$ such that

$$
H\left(A_{k}^{-i}, 1\right) \geqslant-i S_{A_{k}}+b_{k} \text { and } H\left(D_{k}^{i}, 1\right) \geqslant i s_{D_{k}}+b_{k} \text { for every } i \geqslant 0 \text {. }
$$


Combining them, we have

$$
\begin{aligned}
H\left(D_{k}^{i} C_{k} A_{k}^{-i-1}, 1\right) \geqslant H\left(D_{k}^{i}, 1\right)+ & H\left(C_{k}, 1\right)+H\left(A_{k}^{-i-1}, 1\right) \\
& \geqslant i s_{D_{k}}+b_{k}+\lambda_{s}+k \epsilon-(i+1) S_{A_{k}}+b_{k} \stackrel{i \rightarrow \infty}{\longrightarrow} \infty .
\end{aligned}
$$

Therefore, the series $X_{k}=-\sum_{i=0}^{\infty} D_{k}^{i} C_{k} A_{k}^{-i-1}$ converges and hence $X_{k}$ is well-defined.

By Lemma 2.2.15, we have $H\left(A_{k}^{-i-1}, 1\right) \geqslant-(i+1) \lambda_{s}$. Since $H\left(C_{k}, 1\right) \geqslant \lambda_{s}+k \epsilon$ and $H\left(D_{k}, 1\right) \geqslant \lambda_{s+1} \geqslant \lambda_{s}$, we have

$$
H\left(D_{k}^{i} C_{k} A_{k}^{-i-1}, 1\right) \geqslant i \lambda_{s}+\lambda_{s}+k \epsilon-(i+1) \lambda_{s}=k \epsilon,
$$

and hence $H\left(X_{k}, 1\right) \geqslant k \epsilon$, which proves $(i)$.

From $X_{k} A_{k}+C_{k}-D_{k} X_{k}=0$, we have

$$
M_{k+1}=\left[\begin{array}{cc}
A_{k}-B_{k} X_{k} & B_{k} \\
-X_{k} B_{k} X_{k} & D_{k}+X_{k} B_{k}
\end{array}\right] .
$$

Since $A_{k}$ is strictly $\underline{\lambda}^{[s]}$-Hodge bounded and $B_{k}$ is $\underline{\lambda}^{[s]}$-Hodge bounded, we have $A_{k}^{-1} B_{k} \in$ $\mathrm{M}_{s \times(n-s)}\left(\mathbb{F}_{p} \llbracket T \rrbracket\right)$. Combined with $X_{k} B_{k}=-\sum_{i=0}^{\infty} D_{k}^{i} C_{k} A_{k}^{-i}\left(A_{k}^{-1} B_{k}\right)$ and

$$
H\left(D_{k}^{i} C_{k} A_{k}^{-i}\left(A_{k}^{-1} B_{k}\right), 1\right) \geqslant i \lambda_{s}+\lambda_{s}+k \epsilon-i \lambda_{s}+0=\lambda_{s}+k \epsilon \text { for every } i \geqslant 0,
$$

this implies

$$
H\left(X_{k} B_{k}, 1\right) \geqslant \lambda_{s}+k \epsilon
$$

and hence

$$
H\left(C_{k+1}, 1\right)=H\left(-X_{k} B_{k} X_{k}, 1\right) \geqslant \lambda_{s}+k \epsilon+\epsilon=\lambda_{s}+(k+1) \epsilon .
$$

This completes the proof of $(i i)$.

By Lemma 2.2.5. to show that the matrix $\left[\begin{array}{cc}I_{s} & 0 \\ X_{k} & I_{n-s}\end{array}\right]$ is $\underline{\lambda}^{[n]}$-stable, it is enough to show that the matrix

$$
\left[\begin{array}{cc}
D\left(\underline{\lambda}^{[s]}\right) & 0 \\
0 & D\left(\underline{\lambda}^{(s, n]}\right)
\end{array}\right]^{-1}\left[\begin{array}{cc}
I_{s} & 0 \\
X_{k} & I_{n-s}
\end{array}\right]\left[\begin{array}{cc}
D\left(\underline{\lambda}^{[s]}\right) & 0 \\
0 & D\left(\underline{\lambda}^{(s, n]}\right)
\end{array}\right]=\left[\begin{array}{cc}
I_{s} & 0 \\
D\left(\underline{\lambda}^{(s, n]}\right)^{-1} X_{k} D\left(\underline{\lambda}^{[s]}\right) & I_{n-s}
\end{array}\right]
$$

belongs to $\mathrm{M}_{n}\left(\mathbb{F}_{p} \llbracket T \rrbracket\right)$, or equivalently,

$$
D\left(\underline{\lambda}^{(s, n]}\right)^{-1} X_{k} D\left(\underline{\lambda}^{[s]}\right) \in \mathrm{M}_{(n-s) \times s}\left(\mathbb{F}_{p} \llbracket T \rrbracket\right) .
$$

Note that

$$
D\left(\underline{\lambda}^{(s, n]}\right)^{-1} X_{k} D\left(\underline{\lambda}^{[s]}\right)=-\sum_{i=0}^{\infty} D\left(\underline{\lambda}^{(s, n]}\right)^{-1} D_{k}^{i} C_{k} A_{k}^{-i} A_{k}^{-1} D\left(\underline{\lambda}^{[s]}\right) .
$$

Since $A_{k}$ is strictly $\underline{\lambda}^{[s]}$-Hodge bounded, we have

$$
A_{k}^{-1} D\left(\underline{\lambda}^{[s]}\right) \in \mathrm{GL}_{s}\left(\mathbb{F}_{p} \llbracket T \rrbracket\right), \quad\left(\text { i.e. } H\left(A_{k}^{-1} D\left(\underline{\lambda}^{[s]}\right), 1\right) \geqslant 0\right) .
$$

Combined with

$$
H\left(D_{k}^{i-1} C_{k} A_{k}^{-i}, 1\right) \geqslant(i-1) \lambda_{s}+\lambda_{s}+k \epsilon-i \lambda_{s}=k \epsilon>0 \text { for every } i \geqslant 1
$$

and

we have

$$
H\left(D\left(\underline{\lambda}^{(s, n]}\right)^{-1} D_{k}, 1\right) \geqslant 0
$$

$$
D\left(\underline{\lambda}^{(s, n]}\right)^{-1} D_{k}^{i} C_{k} A_{k}^{-i} A_{k}^{-1} D\left(\underline{\lambda}^{[s]}\right) \in \mathrm{M}_{(n-s) \times s}(\mathbb{F} p \llbracket T \rrbracket) \text { for every } i \geqslant 1 \text {. }
$$


Note that by $H\left(D\left(\underline{\lambda}^{(s, n]}\right)^{-1} C_{k}, 1\right) \geqslant 0$, the belonging relation 2.10 holds for $i=0$ as well. Therefore, further combining it with $(2.9)$, we complete the proof of (iii).

Since $M_{k}$ satisfies the hypothesis (1) and conjugating by matrices in $\mathrm{GL}_{n}\left(\mathbb{F}_{p} \llbracket T \rrbracket\right)$ does not change either its Newton or Hodge polygons, we prove the hypothesis (1) for $M_{k+1}$.

The hypothesis (2) for $M_{k+1}$ follows directly from (ii).

Now we are left to prove hypothesis (3) for $M_{k+1}$. Note that

$$
A_{k+1}=A_{k}+B_{k} X_{k}=A_{k}\left(I_{s}+A_{k}^{-1} B_{k} X_{k}\right) .
$$

From our previous discussion, we know that $A_{k}^{-1} B_{k} \in \mathrm{M}_{s \times(n-s)}\left(\mathbb{F}_{p} \llbracket T \rrbracket\right)$. Combined with hypothesis (2) on $M_{k}$ and Lemma 2.2.16, this implies $H\left(A_{k}^{-1} B_{k} X_{k}, 1\right) \geqslant \epsilon>0$ and that $I_{s}+A_{k}^{-1} B_{k} X_{k}$ is invertible. Therefore, $A_{k+1}$ is strictly $\underline{\lambda}^{[s]}-$ Hodge bounded, which proves (iv).

Note that the matrix $M_{1}:=M$ satisfies all the conditions in our claim for $k=1$. Therefore, inductively using this claim, we obtain two infinite sequences of matrices $\underline{X} \in$ $\mathrm{M}_{(n-s) \times s}\left(\mathbb{F}_{p} \llbracket T \rrbracket\right)$ and $\underline{M} \in \mathrm{M}_{(n-s) \times n}\left(\mathbb{F}_{p} \llbracket T \rrbracket\right)$ such that

- $H\left(X_{k}, 1\right) \geqslant k \epsilon$;

- $M_{k}:=s\left[\begin{array}{c:c}A_{k} & B_{k} \\ \hdashline C_{k} & D_{k}\end{array}\right]=Y_{k} M_{k-1} Y_{k}^{-1}$, where $Y_{k}:=\left[\begin{array}{cc}I_{s} & 0 \\ X_{k} & I_{n-s}\end{array}\right]$;

- $Y_{k}$ is $\underline{\lambda}^{[n]}$-stable;

- $H\left(C_{k}, 1\right) \geqslant \lambda_{s}+k \epsilon$ for all $k \geqslant 1$.

Notice that

$$
Y_{k} Y_{k-1} \ldots Y_{1}=\left[\begin{array}{cc}
I_{s} & 0 \\
\sum_{i=1}^{k} X_{i} & I_{n-s}
\end{array}\right]
$$

Since $H\left(X_{k}, 1\right) \geqslant k \epsilon$, the series $\sum_{k=1}^{\infty} X_{k}$ converges to a matrix $X \in \mathrm{M}_{(n-s) \times s}\left(\mathbb{F}_{p} \llbracket T \rrbracket\right)$. From the above construction, we see that $X$ satisfies all the required properties.

Now we state the Newton-Hodge decomposition theorem for matrices in $\mathrm{M}_{\infty}\left(\mathbb{F}_{p} \llbracket T \rrbracket\right)$.

Theorem 2.2.18. Let $M \in M_{\infty}\left(\mathbb{F}_{p} \llbracket T \rrbracket\right)$ be a $\underline{\lambda}$-Hodge bounded matrix and $\Omega=\left\{0=s_{0}<\right.$ $\left.s_{1}<\cdots\right\}$ be an infinite subset of touching vertices of $M$. If

$$
H(M, s)=\sum_{i=1}^{s} \lambda_{i} \text { for all } s \in \Omega
$$

then there exists $W \in \mathrm{GL}_{\infty}\left(\mathbb{F}_{p} \llbracket T \rrbracket\right)$ such that

(1) $W$ is $\underline{\lambda}$-stable, and in particular $W M W^{-1}$ is $\underline{\lambda}$-Hodge bounded.

(2) $W M W^{-1}$ is a block upper triangular matrix of the form

$$
W M W^{-1}=s_{1}\left[\begin{array}{c:c}
N_{11} & N_{12} \\
\hdashline 0 & N_{22}
\end{array}\right] .
$$

Remark 2.2.19. (1) Although the conclusion of Theorem 2.2 .18 only refers to the touching vertex $s_{1}$, the assumption that there exist infinitely many touching vertices of $M$ is necessary, at least according to our proof. The reason is because in the Newton-Hodge Decomposition Theorem 2.2.10 for a finite matrix $M \in \mathrm{M}_{n}\left(\mathbb{F}_{p} \llbracket T \rrbracket\right)$, 
we have an automatic condition $H(M, n)=N(M, n)$, i.e. $(n, N(M, n))$ is always a touching vertex of $M$, while we have no such condition for infinite matrix $M \in \mathrm{M}_{\infty}\left(\mathbb{F}_{p} \llbracket T \rrbracket\right)$. Therefore we have to impose extra condition on the infinite $M$ to obtain the desired decomposition. Here we assume that $M$ has infinitely many touching vertices, which holds in our applications. It is an interesting question whether this assumption can be weakened.

(2) Since $\lim _{n \rightarrow \infty} \lambda_{n}=\infty$, replacing $\Omega$ by a subset if necessary, we can assume that $\lambda_{s_{1}}<$ $\lambda_{s_{2}}$. We will make this assumption throughout the proof of Theorem 2.2.18.

Remark 2.2.20. One must be careful when compute the infinite product of infinite matrices. It is not true in general that the product of an countable infinitely many invertible matrices is invertible, even if the product is well-defined. For example, we denote by $Q_{n} \in \mathrm{GL}_{\infty}\left(\mathbb{F}_{p} \llbracket T \rrbracket\right)$ the infinite matrix obtained by switching the 1 -st and $n$-th rows of the identity matrix $\mathrm{I}_{\infty}$. In particular, set $Q_{1}=\mathrm{I}_{\infty}$. Then the sequence $\left(Q_{n} \cdots Q_{1} \mid n \geqslant 1\right)$ converges to the infinite matrix $\left[\begin{array}{cc}0 & 0 \\ \mathrm{I}_{\infty} & 0\end{array}\right]$, which is obviously not invertible. What we will use is the following result:

Let $\left\{Q_{n}\right\}_{n \geqslant 1}$ be a family of matrices in $\mathrm{GL}_{\infty}\left(\mathbb{F}_{p} \llbracket T \rrbracket\right)$. Assume that there exists a sequence of integers $\left(\alpha_{n}\right)_{n \geqslant 1}$ such that $\lim _{n \rightarrow \infty} \alpha_{n}=\infty$ and $Q_{n}-\mathrm{I}_{\infty} \in T^{\alpha_{n}} \mathrm{M}_{\infty}\left(\mathbb{F}_{p} \llbracket T \rrbracket\right)$. Then the sequence $\left(Q_{n} \cdots Q_{1} \mid n \geqslant 1\right)$ converges to a matrix $Q \in \mathrm{GL}_{\infty}\left(\mathbb{F}_{p} \llbracket T \rrbracket\right)$. In fact, the condition on $Q_{n}$ 's implies that $Q_{n}^{-1}-\mathrm{I}_{\infty} \in T^{\alpha_{n}} \mathrm{M}_{\infty}\left(\mathbb{F}_{p} \llbracket T \rrbracket\right)$ and hence $\left(Q_{1}^{-1} \cdots Q_{n}^{-1} \mid n \geqslant 1\right)$ converges to a matrix which gives the two-sided inverse of $Q$. Moreover, if $Q_{n}$ is $\underline{\lambda}$-stable for all $n \geqslant 1$, the matrix $Q$ is also $\underline{\lambda}$-stable.

Lemma 2.2.21. Let $M$ be the matrix in Theorem 2.2.18, Then

(1) for every $k \geqslant 1$ there exists a $\underline{\lambda}$-stable matrix $P_{k} \in \mathrm{GL}_{\infty}\left(\mathbb{F}_{p} \llbracket T \rrbracket\right)$ such that if we write

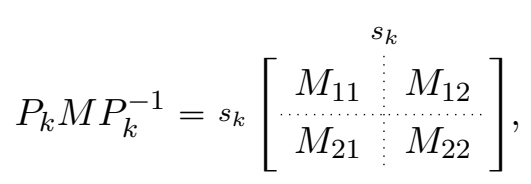

then $v_{T}\left(\operatorname{det}\left(M_{11}\right)\right)=\sum_{i=1}^{s_{k}} \lambda_{i}$.

(2) There exists an integer $\ell_{M}$ such that for every $n \geqslant \ell_{M}$ and every $\underline{\lambda}$-stable matrix $P \in$ $\mathrm{GL}_{\infty}\left(\mathbb{F}_{p} \llbracket T \rrbracket\right)$ the Newton polygon of $\left(P M P^{-1}\right)_{[n]}$ coincides with the Newton polygon of $M$ when one restricts the range of the $x$-coordinates to $\left[0, s_{2}\right]$. In particular, $s_{1}$ is a touching vertex of $M_{[n]}$.

Proof. (1) Since $s_{k}$ is a touching vertex of $M$, we have

$$
N\left(M, s_{k}\right)=H\left(M, s_{k}\right)=\sum_{i=1}^{s_{k}} \lambda_{i} .
$$

Let $m_{1}:=\min \left\{j \mid \lambda_{j}=\lambda_{s_{k}}\right\}$ and $m_{2}:=\max \left\{j \mid \lambda_{j}=\lambda_{s_{k}}\right\}$. Since $M$ is $\underline{\lambda}$-Hodge bounded, there exists a principal $s_{k} \times s_{k}$ minor $N$ of $M$ such that $v_{T}(\operatorname{det}(N))=\sum_{i=1}^{s_{k}} \lambda_{i}$. Moreover, $N$ contains $M_{\left[m_{1}-1\right]}$ as a principal minor and is contained in the principal minor $M_{\left[m_{2}\right]}$. So there exists a permutation matrix $P^{\prime} \in \mathrm{GL}_{m_{2}-m_{1}}\left(\mathbb{F}_{p} \llbracket T \rrbracket\right)$ (that is, a matrix obtained by permuting the row vectors of the identity matrix) such that if we set

$$
N^{\prime}:=\left[\begin{array}{cc}
I_{m_{1}} & 0 \\
0 & P^{\prime}
\end{array}\right] M_{\left[m_{2}\right]}\left[\begin{array}{cc}
I_{m_{1}} & 0 \\
0 & P^{\prime}
\end{array}\right]^{-1}
$$


then $N_{\left[s_{k}\right]}^{\prime}=N$. Set

$$
P:=\left[\begin{array}{ccc}
I_{m_{1}} & 0 & 0 \\
0 & P^{\prime} & 0 \\
0 & 0 & I_{\infty}
\end{array}\right]
$$

By Lemma 2.2.5, $P$ is $\underline{\lambda}$-stable. Clearly, the matrix $P$ has the desired property.

(2) From our hypothesis $\lim _{n \rightarrow \infty} \lambda_{n}=\infty$, there exists an integer $\ell_{M}>s_{2}$ such that $\lambda_{\ell_{M}}>$ $\sum_{i=1}^{s_{2}} \lambda_{i}$, and $\left(\ell_{M}, \sum_{i=1}^{\ell_{M}} \lambda_{i}\right)$ lies above the straight line determined by the line segment in the Hodge polygon of $M$ with slope $\lambda_{s_{2}}$. Combined with that for every $\underline{\lambda}$-stable matrix $P \in$ $\mathrm{GL}_{\infty}\left(\mathbb{F}_{p} \llbracket T \rrbracket\right), P M P^{-1}$ has the same Newton polygon to $M$ and is $\underline{\lambda}$-Hodge bounded, these hypotheses on $\ell_{M}$ implies that for every $n \geqslant \ell_{M}$ and for every $0 \leqslant j<s_{2}$ we have

$$
N\left(\left(P M P^{-1}\right)_{[n]}, j\right) \begin{cases}=N\left(P M P^{-1}, j\right)=N(M, j), & \text { if } N(M, j) \leqslant \sum_{i=1}^{s_{2}} \lambda_{i}, \\ >\sum_{i=1}^{s_{2}} \lambda_{i}, & \text { else, }\end{cases}
$$

and

$$
N\left(\left(P M P^{-1}\right)_{[n]}, s_{2}\right)=N\left(P M P^{-1}, s_{2}\right)=N\left(M, s_{2}\right)=\sum_{i=1}^{s_{2}} \lambda_{i},
$$

and hence complete the proof.

Notation 2.2.22. From now on, we denote by $s$ a fixed vertex of the Newton polygon of $M$ such that $s \geqslant \ell_{M}$ as in Lemma 2.2.21. We also set $\Delta:=\lambda_{s}-\lambda_{s_{1}}$.

Lemma 2.2.23. Under the notations and conditions in Theorem 2.2.18, there exists a $\underline{\lambda}$-stable matrix $Q \in \mathrm{GL}_{\infty}\left(\mathbb{F}_{p} \llbracket T \rrbracket\right)$ such that if we set

$$
Q M Q^{-1}:=s_{1}\left[\begin{array}{c:c}
A_{11} & A_{12} \\
\hdashline A_{21} & A_{22}
\end{array}\right],
$$

then we have $H\left(A_{21}, 1\right) \geqslant \lambda_{s}$ and $A_{11}$ is strictly $\lambda^{\left[s_{1}\right]}$-Hodge bounded.

Proof. By Lemma 2.2.21(1), we may assume $v_{T}\left(\operatorname{det}\left(M_{[s]}\right)\right)=\sum_{i=1}^{s} \lambda_{i}$. Therefore, $M_{[s]}$ satisfies all the conditions in Theorem 2.2.12. By Theorem 2.2.12, there exists a $\underline{\lambda}^{[s]}$-stable matrix $P$ such that $P M_{[s]} P^{-1}$ is of the form

$$
P M_{[s]} P^{-1}=s_{1}\left[\begin{array}{c:c}
B_{11} & B_{12} \\
\hdashline 0 & B_{22}
\end{array}\right] \text {, }
$$

and $B_{11}$ is strictly $\lambda^{\left[s_{1}\right]}$-Hodge bounded. Let $Q:=\left[\begin{array}{cc}P & 0 \\ 0 & I_{\infty}\end{array}\right]$. Clearly, $Q$ is $\underline{\lambda}$-stable as $P$ is $\underline{\lambda}^{[s]}$-stable. Since $M$ is $\underline{\lambda}$-Hodge bounded, the constructed matrix $Q$ satisfies the required properties.

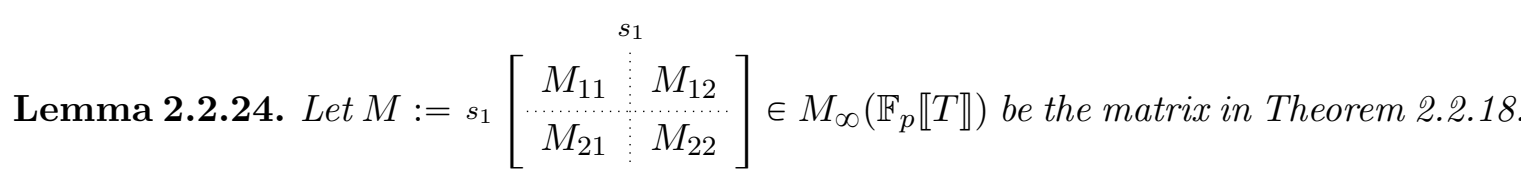
If $M_{11}$ is strictly $\lambda^{\left[s_{1}\right]}$-Hodge bounded and $H\left(M_{21}, 1\right) \geqslant \lambda_{s}+i \Delta$ for some integer $i \geqslant 0$, then there exists a $\underline{\lambda}$-stable matrix $Q:=\left[\begin{array}{cc}I_{s_{1}} & 0 \\ Q^{\prime} & I_{\infty}\end{array}\right] \in \mathrm{GL}_{\infty}\left(\mathbb{F}_{p} \llbracket T \rrbracket\right)$ such that 
- $H\left(Q^{\prime}, 1\right) \geqslant(i+1) \Delta$, and

- if we set

$$
Q M Q^{-1}:=s_{1}\left[\begin{array}{c:c}
A_{11} & A_{12} \\
\hdashline A_{21} & A_{22}
\end{array}\right],
$$

then $H\left(A_{21}, 1\right) \geqslant \lambda_{s}+(i+1) \Delta$.

Proof. We set $s^{\prime}:=s-s_{1}$ and rewrite $M$ as

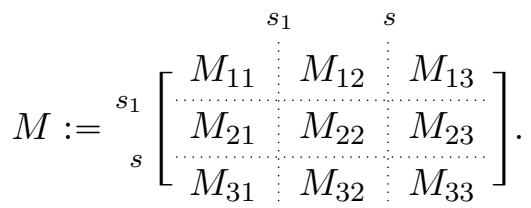

Let

$$
P_{1}:=\left[\begin{array}{ccc}
I_{s_{1}} & 0 & 0 \\
0 & I_{s^{\prime}} & 0 \\
-M_{31} M_{11}^{-1} & 0 & I_{\infty}
\end{array}\right] .
$$

Then we have

$$
\begin{aligned}
M^{\prime}:= & {\left[\begin{array}{lll}
M_{11}^{\prime} & M_{12}^{\prime} & M_{13}^{\prime} \\
M_{21}^{\prime} & M_{22}^{\prime} & M_{23}^{\prime} \\
M_{31}^{\prime} & M_{32}^{\prime} & M_{33}^{\prime}
\end{array}\right]=P_{1} M P_{1}^{-1} } \\
& =\left[\begin{array}{ccc}
M_{11}+M_{13} M_{31} M_{11}^{-1} & M_{12} & M_{13} \\
M_{21}+M_{23} M_{31} M_{11}^{-1} & M_{11} & M_{23} \\
\left(M_{33}-M_{31} M_{11}^{-1} M_{13}\right) M_{31} M_{11}^{-1} & M_{32}-M_{31} M_{11}^{-1} M_{12} & M_{33}-M_{31} M_{11}^{-1} M_{13}
\end{array}\right] .
\end{aligned}
$$

From our hypotheses that $M_{11}$ is strictly $\underline{\lambda}^{\left[s_{1}\right]}$-Hodge bounded and that $M_{12}, M_{13}$ are $\underline{\lambda}^{\left[s_{1}\right]}$-Hodge bounded, we have $M_{11}^{-1} M_{12} \in \mathrm{M}_{s_{1} \times s}\left(\mathbb{F}_{p} \llbracket T \rrbracket\right)$ and $M_{11}^{-1} M_{13} \in \mathrm{M}_{s_{1} \times \infty}\left(\mathbb{F}_{p} \llbracket T \rrbracket\right)$. Combining $H\left(M_{31}, 1\right) \geqslant \lambda_{s}+i \Delta$ with Lemma 2.2.15, we have

$$
H\left(M_{31} M_{11}^{-1}, 1\right) \geqslant \lambda_{s}+i \Delta-\lambda_{s_{1}}=(i+1) \Delta .
$$

Therefore, $M^{\prime}$ is $\underline{\lambda}$-Hodge bounded; and we have the estimations

$$
H\left(M_{21}+M_{23} M_{31} M_{11}^{-1}, 1\right) \geqslant \lambda_{s}+i \Delta
$$

and

$$
\begin{aligned}
& H\left(M_{31}^{\prime}, 1\right)=H\left(\left(-M_{31} M_{11}^{-1} M_{13}+M_{33}\right) M_{31} M_{11}^{-1}, 1\right) \\
& \quad \geqslant H\left(-M_{31} M_{11}^{-1} M_{13}+M_{33}, 1\right)+H\left(M_{31} M_{11}^{-1}, 1\right) \geqslant \lambda_{s}+(i+1) \Delta .
\end{aligned}
$$

By Lemmas 2.2.16 and 2.2.21(2), the matrix $M_{[s]}^{\prime}=\left[\begin{array}{ll}M_{11}^{\prime} & M_{12}^{\prime} \\ M_{21}^{\prime} & M_{22}^{\prime}\end{array}\right]$ satisfies all the conditions in Lemma 2.2 .17 with $n:=s$ and $s:=s_{1}$. Therefore, there exists a matrix $X \in \mathrm{M}_{s \times s_{1}}(\mathbb{F} p \llbracket T \rrbracket)$ such that

(1) $H(X, 1) \geqslant H\left(M_{21}^{\prime}, 1\right)-\lambda_{s_{1}} \geqslant \lambda_{s}+i \Delta-\lambda_{s_{1}}=(i+1) \Delta$

(2) The matrix $\left[\begin{array}{cc}I_{s_{1}} & 0 \\ X & I_{s}\end{array}\right]$ is $\underline{\lambda}^{[s]}$-stable;

(3) $\left[\begin{array}{cc}I_{s_{1}} & 0 \\ X & I_{s}\end{array}\right]\left[\begin{array}{ll}M_{11}^{\prime} & M_{12}^{\prime} \\ M_{21}^{\prime} & M_{22}^{\prime}\end{array}\right]\left[\begin{array}{cc}I_{s_{1}} & 0 \\ -X & I_{s}\end{array}\right]=\left[\begin{array}{cc}M_{11}^{\prime \prime} & M_{12}^{\prime \prime} \\ 0 & M_{22}^{\prime \prime}\end{array}\right]$. 
Set $P_{2}:=\left[\begin{array}{ccc}I_{s_{1}} & 0 & 0 \\ X & I_{s} & 0 \\ 0 & 0 & I_{\infty}\end{array}\right]$. Then $P_{2} \in \mathrm{M}_{\infty}\left(\mathbb{F}_{p} \llbracket T \rrbracket\right)$ is $\underline{\lambda}$-stable and

$$
P_{2} M P_{2}^{-1}:=\left[\begin{array}{ccc}
M_{11}^{\prime \prime} & M_{12}^{\prime \prime} & M_{13}^{\prime} \\
0 & M_{22}^{\prime \prime} & M_{23}^{\prime}+X M_{33}^{\prime} \\
M_{31}^{\prime}-X M_{32}^{\prime} & M_{32}^{\prime} & M_{33}^{\prime} .
\end{array}\right] .
$$

Combining the inequalities

$$
H\left(X M_{32}^{\prime}, 1\right) \geqslant H(X, 1)+H\left(M_{32}^{\prime}, 1\right) \geqslant(i+1) \Delta+\lambda_{s} \text { and } H\left(M_{31}^{\prime}, 1\right) \geqslant \lambda_{s}+(i+1) \Delta,
$$

we have $H\left(M_{31}^{\prime}-X M_{32}^{\prime}, 1\right) \geqslant \lambda_{s}+(i+1) \Delta$.

Setting

$$
Q:=P_{2} P_{1}=\left[\begin{array}{ccc}
I_{s_{1}} & 0 & 0 \\
X & I_{s} & 0 \\
-M_{31} M_{11}^{-1} & 0 & I_{\infty}
\end{array}\right],
$$

from the above discussion we know that $Q$ satisfies all the required properties.

Now we can prove Theorem 2.2.18, which is an easy consequence of the above lemmas.

Proof of Theorem 2.2.18. By Lemma 2.2.23, there exists a $\underline{\lambda}$-stable matrix $Q_{0} \in \mathrm{GL}_{\infty}\left(\mathbb{F}_{p} \llbracket T \rrbracket\right)$ such that if we write

$$
M_{0}:=Q_{0} M Q_{0}^{-1}=s_{1}\left[\begin{array}{c:c}
M_{0,11} & M_{0,12} \\
\hdashline M_{0,21} & M_{0,22}
\end{array}\right],
$$

then $H\left(M_{0,21}, 1\right) \geqslant \lambda_{s}=\lambda_{s}+0 \cdot \Delta$ and $M_{0,11}$ is strictly $\lambda^{\left[s_{1}\right]}$-Hodge bounded. Then we can apply Lemma 2.2 .24 inductively on $k$, and get a sequence of $\underline{\lambda}$-stable matrices

$$
\left(Q_{k}=\left[\begin{array}{cc}
I_{s_{1}} & 0 \\
Q_{k}^{\prime} & I_{\infty}
\end{array}\right] \in \mathrm{GL}_{\infty}\left(\mathbb{F}_{p} \llbracket T \rrbracket\right) \mid k \geqslant 1\right)
$$

with $H\left(Q_{k}^{\prime}, 1\right) \geqslant(k+1) \Delta$ such that we write

$$
M_{k}:=\left(Q_{k} \ldots Q_{1} Q_{0}\right) M\left(Q_{k} \ldots Q_{1} Q_{0}\right)^{-1}=s_{1}\left[\begin{array}{c:c}
M_{k, 11} & M_{k, 12} \\
\hdashline M_{k, 21} & M_{k, 22}
\end{array}\right],
$$

then $H\left(M_{k, 21}, 1\right) \geqslant \lambda_{s}+k \Delta$. By Remarks 2.2.19 and 2.2.20, the product $Q_{k} \ldots Q_{1} Q_{0}$ converges as $k \rightarrow \infty$, and we denote by $W$ this limit. Then $W \in \mathrm{GL}_{\infty}\left(\mathbb{F}_{p} \llbracket T \rrbracket\right)$ is $\underline{\lambda}$-stable and

$$
W M W^{-1}=s_{1}\left[\begin{array}{c:c}
N_{11} & N_{12} \\
0 & N_{22}
\end{array}\right] \text {. }
$$

2.3. Newton-Hodge decomposition for matrices over certain non-commutative rings. In this subsection, we will denote by $R$ a ring with unit but not necessarily commutative. Let $\mathfrak{m} \subset R$ be a two-sided ideal, and $\tilde{T} \in R$ a central element which is not a zero divisor of $R$. We assume that

(1) $R$ is complete under the $(\mathfrak{m}, \tilde{T})$-adic topology, where $(\mathfrak{m}, \tilde{T})$ is the (two-sided) ideal of $R$ generated by $\mathfrak{m}$ and $\tilde{T}$, i.e. we have $R \cong \lim _{n \rightarrow \infty} R /(\mathfrak{m}, \tilde{T})^{n}$. 
(2) There exists a surjective ring homomorphism $\pi: R \rightarrow \mathbb{F}_{p} \llbracket T \rrbracket$ with $\operatorname{ker}(\pi)=\mathfrak{m}$ and $\pi(\tilde{T})=T$.

(3) For $x \in R \backslash\{0\}$, we define $v_{\tilde{T}}(x):=\sup \left\{n \mid x \in \tilde{T}^{n} R\right\}$ and $v_{\tilde{T}}(0):=\infty$. Then for each $\bar{x} \in \mathbb{F}_{p} \llbracket T \rrbracket$, there exists $x \in R$ such that $\pi(x)=\bar{x}$ and $v_{\tilde{T}}(x)=v_{T}(\bar{x})$. The element $x$ with such properties is called a special lift of $\bar{x}$.

Remark 2.3.1. (1) The number $v_{\tilde{T}}(x)=\sup \left\{n \mid x \in \tilde{T}^{n} R\right\}$ is finite for $x \neq 0$, and we have the inequality $v_{\tilde{T}}(x) \leqslant v_{T}(\bar{x})$ with $\bar{x}=\pi(x)$.

(2) Since $\tilde{T}$ is central and not a zero divisor of $R$, we can consider the localization $R\left[\frac{1}{\tilde{T}}\right]$ of $R$ with respect to the multiplicative set $\left\{\tilde{T}^{n} \mid n \geqslant 0\right\}$. The natural homomorphism $R \rightarrow R\left[\frac{1}{\tilde{T}}\right]$ is injective.

(3) Since $\tilde{T}$ is not a zero divisor of $R$, if $x \in \tilde{T}^{k} R$, then there exists a unique $x^{\prime} \in R$ with $x=\tilde{T}^{k} x^{\prime}$. We write $x^{\prime}=\tilde{T}^{-k} x$.

Lemma 2.3.2. Let $x \in \tilde{T}^{k} R$ for some $k \geqslant 1$. If $x \in \mathfrak{m}$, then $\tilde{T}^{-k} x \in \mathfrak{m}$.

Proof. We write $x=\tilde{T}^{k} y$ for some $y \in R$. Applying the homomorphism $\pi$ on both sides of this equality, we get $\pi(x)=T^{k} \pi(y)=0$, and hence $\pi(y)=0$. This implies $y \in \mathfrak{m}$.

Definition 2.3.3. For a matrix $\bar{A}=\left(\bar{a}_{i j}\right) \in \mathrm{M}_{m \times n}\left(\mathbb{F}_{p} \llbracket T \rrbracket\right)$, we call $A=\left(a_{i j}\right) \in \mathrm{M}_{m \times n}(R)$ a special lift of $\bar{A}$ if $a_{i j}$ is a special lift of $\bar{a}_{i j}$ for every $1 \leqslant i \leqslant m$ and $1 \leqslant j \leqslant n$.

We denote by $D_{R}\left(\underline{\lambda}^{[n]}\right)\left(\right.$ resp. $\left.D_{R}(\underline{\lambda})\right)$ the diagonal matrix $\operatorname{Diag}\left(\tilde{T}^{\lambda_{1}}, \ldots, \tilde{T}^{\lambda_{n}}\right) \in \mathrm{M}_{n}(R)$ (resp. $\left.\operatorname{Diag}\left(\tilde{T}^{\lambda_{1}}, \ldots\right) \in \mathrm{M}_{\infty}(R)\right)$. For two integers $m<n$ we denote by $D_{R}\left(\underline{\lambda}^{(m, n]}\right)$ the diagonal matrix $\operatorname{Diag}\left(\tilde{T}^{\lambda_{m+1}}, \ldots, \tilde{T}^{\lambda_{n}}\right) \in \mathrm{M}_{n-m}(R)$. Similar to Definition 2.2.3, we define $\underline{\lambda}$-Hodge bounded matrices and $\underline{\lambda}$-stable matrices over $R$ as follows.

Definition 2.3.4. Let $m, n$ be two positive integers.

(1) A matrix $M \in \mathrm{M}_{n \times m}(R)$ is called $\underline{\lambda}^{[n]}$-Hodge bounded with respect to $\tilde{T} \in R$ if $M=$ $D_{R}\left(\underline{\lambda}^{[n]}\right) M^{\prime}$ for some $M^{\prime} \in \mathrm{M}_{n \times m}(R)$. More generally, for two integers $0 \leqslant n_{1}<n_{2}$ a matrix $M \in \mathrm{M}_{\left(n_{2}-n_{1}\right) \times m}(R)$ is called $\underline{\lambda}^{\left(n_{1}, n_{2}\right]}$-Hodge bounded with respect to $\tilde{T} \in R$ if $M=D_{R}\left(\underline{\lambda}^{\left(n_{1}, n_{2}\right]}\right) M^{\prime}$ for some $M^{\prime} \in \mathrm{M}_{\left(n_{2}-n_{1}\right) \times m}(R)$.

(2) A matrix $M \in \mathrm{M}_{\infty}(R)$ is called $\underline{\lambda}$-Hodge bounded with respect to $\tilde{T} \in R$ if $M=$ $D_{R}(\underline{\lambda}) M^{\prime}$ for some $M^{\prime} \in \mathrm{M}_{\infty}(R)$.

(3) A matrix $A \in \mathrm{M}_{n}(R)$ is called $\underline{\lambda}^{[n]}$-stable with respect to $\tilde{T} \in R$ if for every $\underline{\lambda}^{[n]}$ Hodge bounded matrix $B \in \mathrm{M}_{n}(R), A B$ is also $\underline{\lambda}^{[n]}$-Hodge bounded.

(4) A matrix $A \in \mathrm{M}_{\infty}(R)$ is called $\underline{\lambda}$-stable with respect to $\tilde{T} \in R$ if for every $\underline{\lambda}$-Hodge bounded matrix $B \in \mathrm{M}_{\infty}(R), A \bar{B}$ is also $\underline{\lambda}$-Hodge bounded.

Convention 2.3.5. In the rest of this section, we will fix an element $\tilde{T} \in R$. When we say that a matrix is $\underline{\lambda}^{[n]}$-Hodge bounded or $\underline{\lambda}^{[n]}$-stable, we mean that it is so with respect to $\tilde{T}$.

Since determinants do not behave well for matrices over noncommutative rings, we do not define general Newton functions and Hodge functions for matrices over $R$. But we make the following.

Definition 2.3.6. For a nonzero matrix $A=\left(a_{i, j}\right)_{1 \leqslant i \leqslant m, 1 \leqslant j \leqslant n} \in \mathrm{M}_{m \times n}(R)$, we set

$$
H(A, 1):=\min \left\{v_{\tilde{T}}\left(a_{i, j}\right) \mid 1 \leqslant i \leqslant m, 1 \leqslant j \leqslant n\right\},
$$

which is a well-defined integer. If $H(A, 1) \geqslant k$ for some $k \in \mathbb{Z}_{\geqslant 0}$, we denote by $\tilde{T}^{-k} A$ the (unique) matrix $N$ in $\mathrm{M}_{m \times n}(R)$ satisfying $\tilde{T}^{k} N=A$.

We also have a similar criterion of $\underline{\lambda}$-stability as Lemma 2.2 .5 
Lemma 2.3.7. For $n \in[\infty]$ and a matrix $A=\left(a_{i j}\right) \in \mathrm{M}_{n}(R)$, the following statements are equivalent:

(1) $A$ is $\underline{\lambda}^{[n]}$-stable.

(2) There exists a matrix $B \in \mathrm{M}_{n}(R)$ such that $A D_{R}\left(\underline{\lambda}^{[n]}\right)=D_{R}\left(\underline{(}^{[n]}\right) B$.

(3) For all $i>j$, we have $v_{\tilde{T}}\left(a_{i j}\right) \geqslant \lambda_{i}-\lambda_{j}$, i.e. $a_{i j} \in \tilde{T}^{\lambda_{i}-\lambda_{j}} R$.

The proof is almost identical to that of Lemma 2.2.5 so we omit the proof here, and it has the following direct consequence.

Corollary 2.3.8. Let $\bar{A} \in \mathrm{M}_{n}\left(\mathbb{F}_{p} \llbracket T \rrbracket\right)$ be a $\underline{\lambda}^{[n]}$-stable matrix. If $A \in \mathrm{M}_{n}(R)$ is a special lift of $\bar{A}$, then $A$ is $\underline{\lambda}^{[n]}$-stable.

Lemma 2.3.9. Let $n \in[\infty]$ and $\bar{P} \in \mathrm{GL}_{n}\left(\mathbb{F}_{p} \llbracket T \rrbracket\right)$. Then any lift matrix $P \in \mathrm{M}_{n}(R)$ of $\bar{P}$ is invertible.

Proof. From $\bar{P} \in \mathrm{GL}_{n}\left(\mathbb{F}_{p} \llbracket T \rrbracket\right)$, there exists $\bar{Q} \in \mathrm{GL}_{n}\left(\mathbb{F}_{p} \llbracket T \rrbracket\right)$ such that $\bar{P} \bar{Q}=\bar{Q} \bar{P}=\mathrm{I}_{n}$. Let $Q$ be an arbitrary lift of $\bar{Q}$. Then $B:=\mathrm{I}_{n}-P Q \in \mathrm{M}_{n}(\mathfrak{m})$ and the series $B^{\prime}:=\sum_{i=0}^{\infty} B^{i}$ converges. By a direct computation, we have $P Q B^{\prime}=\mathrm{I}_{n}$. Similarly, let $C:=\mathrm{I}_{n}-Q P \in$ $\mathrm{M}_{n}(\mathfrak{m})$ and the series $C^{\prime}:=\sum_{i=0}^{\infty} C^{i}$ converges. So we have $C^{\prime} Q P=\mathrm{I}_{n}$ and hence $P$ is invertible in $\mathrm{M}_{n}(R)$.

Proposition 2.3.10. Let $n_{1}<n$ be two positive integers, $M:=n_{1}\left[\begin{array}{cc}A & n_{1} \\ \hdashline & B \\ \hdashline & D\end{array}\right], \mathrm{M}_{n}(R)$ be $a \underline{\lambda}^{[n]}-$ Hodge bounded matrix, and $\bar{M} \in \mathrm{M}_{n}\left(\mathbb{F}_{p} \llbracket T \rrbracket\right)$ be its reduction by $\mathfrak{m}$. Fix an integer $\alpha \geqslant \lambda_{n_{1}}$. Assume that

(1) $H(C, 1) \geqslant \alpha$ and $C \equiv 0(\bmod \mathfrak{m})$;

(2) $n_{1}$ is a touching vertex of $\bar{M}$, and $\bar{A}$ is strictly $\underline{\lambda}^{\left[n_{1}\right]}$-Hodge bounded.

Then there exists a matrix $X \in \mathrm{M}_{\left(n-n_{1}\right) \times n_{1}}(\mathfrak{m})$ with $H(X, 1) \geqslant \alpha-\lambda_{n_{1}}$ such that if we denote $Y:=\left[\begin{array}{cc}I_{n_{1}} & 0 \\ X & I_{n-n_{1}}\end{array}\right]$, then $Y$ is $\underline{\lambda}^{[n]}$-stable and $Y M Y^{-1}$ is of the form

$$
Y M Y^{-1}=n_{1}\left[\begin{array}{c:c}
A^{\prime} & \\
A^{\prime} & B^{\prime} \\
0 & D^{\prime}
\end{array}\right] .
$$

First we need the following lemma.

Lemma 2.3.11. Let $M \in \mathrm{M}_{n}(R)$ be the matrix in Proposition 2.3.10. Assume further that $\tilde{T}^{-\alpha} C \equiv 0\left(\bmod \mathfrak{m}^{k}\right)$ for some integer $k \geqslant 1$. Then there exists a matrix $X_{k} \in$ $\mathrm{M}_{\left(n-n_{1}\right) \times n_{1}}(R)$ such that

(1) $H\left(X_{k}, 1\right) \geqslant \alpha-\lambda_{n_{1}}$ and $\tilde{T}^{\lambda_{n_{1}}-\alpha} X_{k} \equiv 0\left(\bmod \mathfrak{m}^{k}\right)$.

(2) The matrix $Y_{k}:=\left[\begin{array}{cc}I_{n_{1}} & 0 \\ X_{k} & I_{n-n_{1}}\end{array}\right]$ is $\underline{\lambda}^{[n]}$-stable.

(3) If we write

$$
Y_{k} M Y_{k}^{-1}:=n_{1}\left[\begin{array}{c:c}
A^{\prime} & B^{\prime} \\
C^{\prime} & D^{\prime}
\end{array}\right]
$$

then $H\left(C^{\prime}, 1\right) \geqslant \alpha$ and $\tilde{T}^{-\alpha} C^{\prime} \equiv 0\left(\bmod \mathfrak{m}^{k+1}\right)$. 
Proof. Since $M$ is $\underline{\lambda}^{[n]}$-Hodge bounded, so is its reduction $\bar{M}$. By Lemma 2.2.14, we have $S_{\bar{A}}<s_{\bar{D}}$, where

$S_{\bar{A}}:=\max \left\{v_{T}(\beta) \mid \beta\right.$ is an eigenvalue of $\left.\bar{A}\right\}$ and $s_{\bar{D}}:=\max \left\{v_{T}(\beta) \mid \beta\right.$ is an eigenvalue of $\left.\bar{D}\right\}$. By Lemma 2.2.13, there exists $b \in \mathbb{R}$ such that for every $i \geqslant 1$ we have

$$
H\left(\bar{A}^{-i}, 1\right) \geqslant-i S_{\bar{A}}+b \text { and } H\left(\bar{D}^{i}, 1\right) \geqslant i s_{\bar{D}}+b .
$$

For every integer $i \geqslant 1$, we set $t_{i}:=i \lambda_{n_{1}}$ and construct matrices as follows.

- Since $T^{t_{i}} \bar{A}^{-i}$ belongs to $\mathrm{M}_{n_{1}}\left(\mathbb{F}_{p} \llbracket T \rrbracket\right)$, we can find a special lift $A^{\prime}(-i) \in \mathrm{M}_{n_{1}}(R)$ of $T^{t_{i}} \bar{A}^{-i}$ and set $A(-i):=\tilde{T}^{-t_{i}} A^{\prime}(-i) \in \mathrm{M}_{n_{1}}\left(R\left[\frac{1}{\tilde{T}}\right]\right)$.

- We fix a special lift $D(i) \in \mathrm{M}_{n-n_{1}}(R)$ of $\bar{D}^{i} \in \mathrm{M}_{n-n_{1}}\left(\mathbb{F}_{p} \llbracket T \rrbracket\right)$.

- To simplify our notations, we set $A(0):=I_{n_{1}} \in \mathrm{M}_{n_{1}}(R)$ and $D(0):=I_{n-n_{1}} \in$ $\mathrm{M}_{n-n_{1}}(R)$.

- Since $A$ is $\underline{\lambda}^{\left[n_{1}\right]}$-Hodge bounded, we can write $A=D_{R}\left(\underline{\lambda}^{\left[n_{1}\right]}\right) \cdot A^{\prime}$ for some $A^{\prime} \in$ $\mathrm{M}_{n_{1}}(R)$. As the reduction $\bar{A}$ of $A$ by $\mathfrak{m}$ is strictly $\underline{\lambda}^{\left[n_{1}\right]}$-Hodge bounded, the reduction $\bar{A}^{\prime}$ of $A^{\prime}$ by $\mathfrak{m}$ is invertible. It follows from Lemma 2.3.9 that $A^{\prime} \in \mathrm{GL}_{n_{1}}(R)$. We define $\tilde{A}^{-1}:=A^{\prime-1} \cdot D_{R}\left(\underline{\lambda}^{\left[n_{1}\right]}\right)^{-1} \in \mathrm{M}_{n_{1}}\left(R\left[\frac{1}{\tilde{T}}\right]\right)$. Although $\tilde{A}^{-1}$ satisfies $A \cdot \tilde{A}^{-1}=$ $\tilde{A}^{-1} \cdot A=I_{n_{1}}, A$ is not invertible in $\mathrm{M}_{n_{1}}(R)$ and $\tilde{A}^{-1}$ is the inverse of $A$ in $\mathrm{M}_{n_{1}}\left(R\left[\frac{1}{\tilde{T}}\right]\right)$. To emphasize this fact, we use the notation $\tilde{A}^{-1}$ instead of $A^{-1}$. Finally we remark that $\tilde{T}^{\lambda_{n_{1}}} \tilde{A}^{-1} \in \mathrm{M}_{n_{1}}(R)$.

The construction above give us

$H\left(\tilde{T}^{t_{i}} A(-i), 1\right)=H\left(A^{\prime}(-i), 1\right)=H\left(T^{t_{i}} \bar{A}^{-i}, 1\right)=t_{i}+H\left(\bar{A}^{-i}, 1\right)$ and $H(D(i), 1)=H\left(\bar{D}^{i}, 1\right)$.

Set

$$
\begin{aligned}
X_{k}:=-\left(C \tilde{A}^{-1}+\sum_{i=1}^{\infty} D D(i-1) C A(-i) \tilde{A}^{-1}\right) & \\
& =-\left(C+\sum_{i=1}^{\infty} D D(i-1) C A(-i)\right) \tilde{A}^{-1} .
\end{aligned}
$$

We first verify that each term $S$ in the sum of (2.11) satisfies $H(S, 1) \geqslant \alpha-\lambda_{n_{1}}$ and in particular belongs to $\mathrm{M}_{\left(n-n_{1}\right) \times n_{1}}(R)$. In fact, for every integer $i \geqslant 1$ we have

$$
\begin{aligned}
& H(D(i-1) C A(-i), 1) \geqslant H(D(i-1), 1)+H(C, 1)+H(A(-i), 1) \\
& =H\left(\bar{D}^{i-1}, 1\right)+H(C, 1)+H\left(\bar{A}^{-i}, 1\right) \geqslant(i-1) \lambda_{n_{1}}+\alpha-i \lambda_{n_{1}}=\alpha-\lambda_{n_{1}},
\end{aligned}
$$

where the second inequality is from Lemma 2.2.15.

Combined with the fact that $\tilde{T}^{-\lambda_{n_{1}}} D \in \mathrm{M}_{n-n_{1}}(R)$ and $\tilde{T}^{\lambda_{n_{1}}} \tilde{A}^{-1} \in \mathrm{M}_{n_{1}}(R)$, this chain of inequality implies $H\left(D D(i-1) C A(-i) \tilde{A}^{-1}, 1\right) \geqslant \alpha-\lambda_{n_{1}}$. On the other hand, from $H(C, 1) \geqslant \alpha \geqslant \lambda_{n_{1}}$ and $\tilde{T}^{\lambda_{n_{1}}} \tilde{A}^{-1} \in \mathrm{M}_{n_{1}}(R)$, we have

$$
C \tilde{A}^{-1} \in \mathrm{M}_{\left(n-n_{1}\right) \times n_{1}}(R) \text { and } H\left(C \tilde{A}^{-1}, 1\right) \geqslant \alpha-\lambda_{n_{1}} .
$$

Now we verify the convergence of the infinite series in 2.11. From $s_{\bar{D}}>S_{\bar{A}}$, we have

$$
\begin{aligned}
H\left(D D(i-1) C A(-i) \tilde{A}^{-1}, 1\right) \geqslant \lambda_{n_{1}}+(i-1) s_{\bar{D}}+ & b+\alpha-i S_{\bar{A}}+b-\lambda_{n_{1}} \\
& =i\left(s_{\bar{D}}-S_{\bar{A}}\right)-s_{\bar{D}}+2 b+\alpha \stackrel{i \rightarrow \infty}{\longrightarrow} \infty .
\end{aligned}
$$

Therefore, the series in 2.11 converges to a matrix $X_{k} \in \mathrm{M}_{\left(n-n_{1}\right) \times n_{1}}(R)$, and

$$
H\left(X_{k}, 1\right) \geqslant \alpha-\lambda_{n_{1}} \text {. }
$$


Moreover, from $\tilde{T}^{-\alpha} C \equiv 0\left(\bmod \mathfrak{m}^{k}\right)$, we have

$$
\tilde{T}^{\lambda_{n_{1}}-\alpha} X_{k} \equiv 0 \quad\left(\bmod \mathfrak{m}^{k}\right) \text { and } \tilde{T}^{-\alpha} X_{k} D_{R}\left(\underline{\lambda}^{\left(n_{1}, n\right]}\right) \equiv 0 \quad\left(\bmod \mathfrak{m}^{k}\right) .
$$

Now we set $Y_{k}:=\left[\begin{array}{cc}I_{n_{1}} & 0 \\ X_{k} & I_{n-n_{1}}\end{array}\right]$, and have

$$
Y_{k} D_{R}\left(\underline{\lambda}^{[n]}\right)=\left[\begin{array}{cc}
D_{R}\left(\underline{\lambda}^{\left[n_{1}\right]}\right) & 0 \\
X_{k} D_{R}\left(\underline{\lambda}^{\left[n_{1}\right]}\right) & D_{R}\left(\underline{\lambda}^{\left(n_{1}, n\right]}\right)
\end{array}\right] .
$$

From $\tilde{A}^{-1}=A^{\prime-1} D_{R}\left(\underline{\underline{\lambda}}^{\left[n_{1}\right]}\right)^{-1}$, we have

$$
X_{k} D_{R}\left(\underline{\lambda}^{\left[n_{1}\right]}\right)=-\left(C+\sum_{i=1}^{\infty} D D(i-1) C A(-i)\right) A^{\prime-1} .
$$

Combined with 2.12 and that both $C$ and $D$ are $\underline{\lambda}^{\left(n_{1}, n\right]}$-Hodge bounded, this equality implies that $X_{k} D_{R}\left(\underline{\lambda}^{\left[n_{1}\right]}\right)$ is $\underline{\lambda}^{\left(n_{1}, n\right]}$-Hodge bounded and

$$
H\left(X_{k} D_{R}\left(\underline{\lambda}^{\left[n_{1}\right]}\right), 1\right) \geqslant \alpha .
$$

Hence there exists $Y_{k}^{\prime} \in \mathrm{M}_{n}(R)$ such that $Y_{k} D_{R}\left(\underline{\lambda}^{[n]}\right)=D_{R}\left(\underline{\lambda}^{[n]}\right) Y_{k}^{\prime}$. By Lemma 2.3.7, $Y_{k}$ is $\underline{\lambda}^{[n]}$-stable.

Note that

$$
Y_{k} M Y_{k}^{-1}=\left[\begin{array}{cc}
A-B X_{k} & B \\
X_{k} A+C-D X_{k}-X_{k} B X_{k} & X_{k} B+D
\end{array}\right] .
$$

We set $C^{\prime}:=X_{k} A+C-D X_{k}-X_{k} B X_{k}$ and need to prove that

$$
H\left(C^{\prime}, 1\right) \geqslant \alpha \text { and } \tilde{T}^{-\alpha} C^{\prime} \equiv 0 \quad\left(\bmod \mathfrak{m}^{k+1}\right) .
$$

The first inequality follows directly from $H(C, 1) \geqslant \alpha,(2.13)$ and $(2.15)$. It remains to prove the second congruence relation. From our construction, for every $i \geqslant 1$ we have

$$
\begin{aligned}
& \tilde{A}^{-1} A \equiv I_{n_{1}} \quad(\bmod \mathfrak{m}) \\
& \tilde{T}^{(i+1) \lambda_{n_{1}}} A(-i) \tilde{A}^{-1} \equiv \tilde{T}^{(i+1) \lambda_{n_{1}}} A(-i-1) \tilde{A}^{-1} A \quad(\bmod \mathfrak{m}), \\
& \tilde{T}^{-(i+1) \lambda_{n_{1}}} D^{2} D(i-1) \equiv \tilde{T}^{-(i+1) \lambda_{n_{1}}} D D(i) \quad(\bmod \mathfrak{m}) .
\end{aligned}
$$

Combining these congruence relations above with $\tilde{T}^{-\alpha} C \equiv 0\left(\bmod \mathfrak{m}^{k}\right)$, we have

$$
\tilde{T}^{-\alpha} D^{2} D(i-1) C A(-i) \tilde{A}^{-1} \equiv \tilde{T}^{-\alpha} D D(i) C A(-i-1) \tilde{A}^{-1} A \quad\left(\bmod \mathfrak{m}^{k+1}\right)
$$

and

$$
\tilde{T}^{-\alpha} C \equiv \tilde{T}^{-\alpha} C \tilde{A}^{-1} A \quad\left(\bmod \mathfrak{m}^{k+1}\right) .
$$

By $\tilde{T}^{\lambda_{n_{1}}} A(-1) \tilde{A}^{-1} A \equiv \tilde{T}^{\lambda_{n_{1}}} \tilde{A}^{-1}(\bmod \mathfrak{m})$, we have

$$
\tilde{T}^{-\alpha} D C \tilde{A}^{-1} \equiv \tilde{T}^{-\alpha} D C A(-1) \tilde{A}^{-1} A \quad\left(\bmod \mathfrak{m}^{k+1}\right) .
$$

By (2.14), we have $X_{k} \equiv 0\left(\bmod \mathfrak{m}^{k}\right)$ and $\tilde{T}^{-\alpha} X_{k} B \equiv 0\left(\bmod \mathfrak{m}^{k}\right)$. Combined with our hypothesis $k \geqslant 1$, the above two congruence relations imply

$$
\tilde{T}^{-\alpha} X_{k} B X_{k} \equiv 0 \quad\left(\bmod \mathfrak{m}^{k+1}\right) .
$$

Combined with (2.11), 2.16, 2.17) and 2.18), this congruence implies

$$
\tilde{T}^{-\alpha} C^{\prime} \equiv 0 \quad\left(\bmod \mathfrak{m}^{k+1}\right) \text {. }
$$


Remark 2.3.12. We emphasize that in the above argument, all the congruence relations modulo powers of $\mathfrak{m}$ are for matrices with entries in $R$ instead of $R\left[\frac{1}{\widetilde{T}}\right]$. The reason is because $\mathfrak{m}^{k} \cdot R\left[\frac{1}{\tilde{T}}\right] \cap R$ and $\mathfrak{m}^{k}$ are not necessary to be the same. Equivalently, we do not know whether $\tilde{T}$ is a non-zero divisor in the ring $R / \mathfrak{m}^{k}$ in general. However, this is true for $k=1$ by Lemma 2.3 .2 .

Now we prove Proposition 2.3.10.

Proof of Proposition 2.3.10. Combining our hypotheses $C \equiv 0(\bmod \mathfrak{m})$ and $H(C, 1) \geqslant \alpha$ with Lemma 2.3.2, we have $\tilde{T}^{-\alpha} C \equiv 0(\bmod \mathfrak{m})$. We can apply Lemma 2.3.11 inductively to get a sequence of matrices $\left(X_{k}\right)_{k \geqslant 1}$ in $\mathrm{M}_{\left(n-n_{1}\right) \times n_{1}}(R)$ with the following properties:

- $H\left(X_{k}, 1\right) \geqslant \alpha-\lambda_{n_{1}}$, and $\tilde{T}^{\lambda_{n_{1}}-\alpha} X_{k} \equiv 0\left(\bmod \mathfrak{m}^{k}\right)$.

- The matrix $Y_{k}=\left[\begin{array}{cc}I_{n_{1}} & 0 \\ X_{k} & I_{n-n_{1}}\end{array}\right]$ is $\underline{\lambda}^{[n]}$-stable.

- If we set

$$
\left(Y_{k} \ldots Y_{1}\right) M\left(Y_{k} \ldots Y_{1}\right)^{-1}:=n_{1}\left[\begin{array}{c:c}
A_{k}^{\prime} & B_{k}^{\prime} \\
\hdashline C_{k}^{\prime} & D_{k}^{\prime}
\end{array}\right]
$$

then $H\left(C_{k}^{\prime}, 1\right) \geqslant \alpha$ and $\tilde{T}^{-\alpha} C_{k}^{\prime} \equiv 0\left(\bmod \mathfrak{m}^{k+1}\right)$.

The series $\sum_{i=1}^{\infty} X_{i}$ converges to a matrix $X \in \mathrm{M}_{\left(n-n_{1}\right) \times n_{1}}(R)$ with $H(X, 1) \geqslant \alpha-\lambda_{n_{1}}$. Therefore the products $\left\{Y_{k} \ldots Y_{1} \mid k \geqslant 1\right\}$ converge to the matrix $Y=\left[\begin{array}{cc}I_{n_{1}} & 0 \\ X & I_{n-n_{1}}\end{array}\right]$. By our construction as above, it is straightforward to verify that $Y$ is $\underline{\lambda}^{[n]}$-stable and $Y M Y^{-1}$ is of the form $n_{1}\left[\begin{array}{c:c}A^{\prime} & B^{\prime} \\ \hdashline 0 & D^{\prime}\end{array}\right]$.

Theorem 2.3.13. Let $M \in \mathrm{M}_{\infty}(R)$ be a $\underline{\lambda}$-Hodge bounded matrix and $\bar{M} \in \mathrm{M}_{\infty}\left(\mathbb{F}_{p} \llbracket T \rrbracket\right)$ be its reduction by $\mathfrak{m}$. Suppose that $\Omega=\left\{s_{1}<s_{2}<\ldots\right\}$ is a set of touching vertices of $\bar{M}$ such that $N(\bar{M}, s)=\sum_{i=1}^{s} \lambda_{i}$, for all $s \in \Omega$. Then there exists a $\underline{\lambda}$-stable matrix $Q \in \mathrm{GL}_{\infty}(R)$ with the following properties:

(1) $Q$ is $\underline{\lambda}$-stable, and in particular $Q M Q^{-1}$ is $\underline{\lambda}$-Hodge bounded; and

(2) $Q M Q^{-1}$ is of the following block upper triangular shape:

$$
Q M Q^{-1}=s_{1}\left[\begin{array}{cc}
M_{11} & M_{12} \\
\hdashline 0 & M_{22}
\end{array}\right]
$$

Proof. By Theorem 2.2.18, there exists a $\underline{\lambda}$-stable matrix $\bar{Q}^{\prime} \in \mathrm{GL}_{\infty}\left(\mathbb{F}_{p} \llbracket T \rrbracket\right)$ such that $\bar{Q}^{\prime} \bar{M} \bar{Q}^{\prime-1}$ is block upper triangular of the shape $s_{1}\left[\begin{array}{c:c}N_{11} & N_{12} \\ 0 & N_{22}\end{array}\right]$. Let $Q^{\prime} \in \mathrm{M}_{\infty}(R)$ be a special lift of $\bar{Q}^{\prime}$. By Lemma 2.3.9, $Q^{\prime}$ is invertible; and by Corollary 2.3.8, $Q^{\prime}$ is $\underline{\lambda}$-stable. Thus we may assume that $\bar{M}$ is block upper triangular with the prescribed shape. We first take an infinite subsequence $\underline{s}^{\prime}:=\left(s_{1}^{\prime}, \ldots\right)$ of $\Omega$ such that $s_{1}^{\prime}>\ell_{\bar{M}}$ as in Lemma 2.2.21. 
By Proposition 2.3.10 with $M:=M_{\left[s_{1}^{\prime}\right]} \in \mathrm{M}_{s_{1}^{\prime}}(R)$, we get a matrix $X_{1} \in \mathrm{M}_{\left(s_{1}^{\prime}-s_{1}\right) \times s_{1}}(R)$ such that

- $H\left(X_{1}, 1\right) \geqslant \lambda_{s_{1}^{\prime}}-\lambda_{s_{1}}$.

- If we set $Y_{1}:=\left[\begin{array}{cc}I_{s_{1}} & 0 \\ X_{1} & I_{s_{1}^{\prime}-s_{1}}\end{array}\right]$, then $Y_{1}$ is $\underline{\lambda}^{\left[s_{1}^{\prime}\right]}$-stable and $Y_{1} M_{\left[s_{1}^{\prime}\right]} Y_{1}^{-1}$ is of the form

$$
s_{1}\left[\begin{array}{c:c}
s_{1}^{\prime} & \\
A_{1}^{\prime} & B_{1}^{\prime} \\
\hdashline 0 & D_{1}^{\prime}
\end{array}\right] .
$$

Let $P_{1}:=\left[\begin{array}{cc}Y_{1} & 0 \\ 0 & I_{\infty}\end{array}\right]=\left[\begin{array}{ccc}I_{s_{1}} & 0 & 0 \\ X_{1} & I_{s_{1}^{\prime}-s_{1}} & 0 \\ 0 & 0 & I_{\infty}\end{array}\right]$. Then $P_{1}$ is $\underline{\lambda}$-stable and $M_{1}:=P_{1} M P_{1}^{-1}$ is of the form $\left[\begin{array}{ccc}A_{1}^{\prime} & B_{1}^{\prime} & * \\ 0 & D_{1}^{\prime} & * \\ * & * & *\end{array}\right]$.

Now we consider the matrix $\left(M_{1}\right)_{\left[s_{2}^{\prime}\right]} \in \mathrm{M}_{s_{2}^{\prime}}(R)$ which satisfies all the hypotheses in Proposition 2.3.10 with $\alpha=\lambda_{s_{2}^{\prime}}$. Moreover, if we write $\left(M_{1}\right)_{\left[s_{2}^{\prime}\right]}$ in the form $\left(M_{1}\right)_{\left[s_{2}^{\prime}\right]}=$ $s_{1}\left[\begin{array}{c:c}A & B \\ \hdashline C & D\end{array}\right]$, then $H(C, 1) \geqslant \lambda_{s_{2}^{\prime}}$. Hence there exists $X_{2} \in \mathrm{M}_{\left(s_{2}^{\prime}-s_{1}\right) \times s_{1}}(\mathfrak{m})$ such that if we set

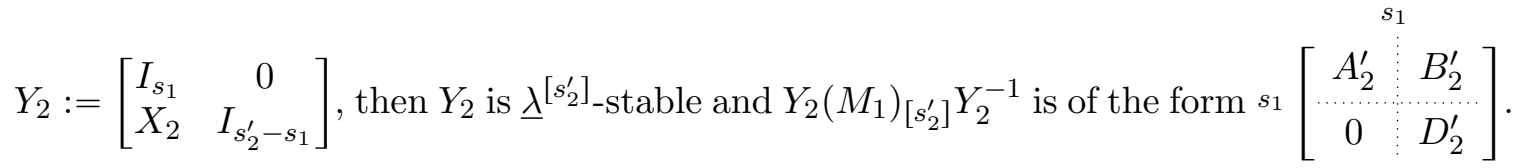
Moreover, we have $H\left(X_{2}, 1\right) \geqslant \lambda_{s_{2}^{\prime}}-\lambda_{s_{1}}$. Let $P_{2}:=\left[\begin{array}{cc}Y_{2} & 0 \\ 0 & I_{\infty}\end{array}\right]=\left[\begin{array}{ccc}I_{s_{1}} & 0 & 0 \\ X_{2} & I_{s_{2}^{\prime}-s_{1}} & 0 \\ 0 & 0 & I_{\infty}\end{array}\right]$. Then $P_{2}$ is $\underline{\lambda}$-stable and $P_{2} M_{1} P_{2}^{-1}=\left(P_{2} P_{1}\right) M\left(P_{2} P_{1}\right)^{-1}$ is of the form $\left[\begin{array}{ccc}A_{2}^{\prime} & B_{2}^{\prime} & * \\ 0 & D_{2}^{\prime} & * \\ * & * & *\end{array}\right]$.

We repeat this iteration and get a sequence of matrices

$$
\left(P_{k}=\left[\begin{array}{cc}
I_{s_{1}} & 0 \\
P_{k}^{\prime} & I_{\infty}
\end{array}\right] \mid k \in \mathbb{N}\right) \subset \mathrm{GL}_{\infty}(R)
$$

such that $P_{k}$ is $\underline{\lambda}$-stable and $H\left(P_{k}^{\prime}, 1\right) \geqslant \lambda_{s_{k}^{\prime}}-\lambda_{s_{1}}$. Moreover, $\left(P_{k} \ldots P_{1}\right) M\left(P_{k} \ldots P_{1}\right)^{-1}$ is of the form $\left[\begin{array}{ccc}A_{k}^{\prime} & B_{k}^{\prime} & * \\ 0 & D_{k}^{\prime} & * \\ * & * & *\end{array}\right]$. The infinite series $\sum_{i=1}^{\infty} P_{i}^{\prime}$ converges to a matrix $P^{\prime}$ and hence the product $P_{k} \ldots P_{1}$ converges to $P=\left[\begin{array}{cc}I_{s_{1}} & 0 \\ P^{\prime} & I_{\infty}\end{array}\right]$. By our construction, $P$ is $\underline{\lambda}$-stable and $P M P^{-1}$ is of the form $s_{1}\left[\begin{array}{c:c}N_{11} & N_{12} \\ \hdashline 0 & N_{22}\end{array}\right]$.

3. Automorphic FORMS For DEFinite quATERnion ALGEBras AND COMPleted HOMOLOGY

\subsection{Notations.}


(1) Let $F$ be a totally real field of degree $g$ over $\mathbb{Q}$ and $\mathcal{O}_{F}$ be its ring of integers. Let $I:=\operatorname{Hom}(F, \overline{\mathbb{Q}})$ denote the set of embeddings of $F$ into $\overline{\mathbb{Q}}$.

(2) Fix an odd prime $p$ which splits completely in $F$. We set $\mathcal{O}_{p}:=\mathcal{O}_{F} \otimes_{\mathbb{Z}} \mathbb{Z}_{p}$ and $F_{p}:=F \otimes_{\mathbb{Q}} \mathbb{Q}_{p}$. For every $i \in I$, let $\mathfrak{p}_{i}$ be the prime of $\mathcal{O}_{F}$ induced by the composite $i_{p}=\iota_{p} \circ i: F \rightarrow \overline{\mathbb{Q}}_{p}$. Let $F_{\mathfrak{p}_{i}}$ (resp. $\mathcal{O}_{\mathfrak{p}_{i}}$ ) denote the completion of $F$ (resp. $\mathcal{O}_{F}$ ) at $\mathfrak{p}_{i}$. For $\alpha \in F_{p}$ and $i \in I$, we denote by $\alpha_{i}$ the $i$-component of $\alpha$ under the natural isomorphism $F_{p} \cong \prod_{i \in I} F_{\mathfrak{p}_{i}}$. Since $p$ splits in $F$, the embedding $\mathbb{Q}_{p} \rightarrow F_{\mathfrak{p}_{i}}$ is an isomorphism $\mathbb{Q}_{p} \cong F_{\mathfrak{p}_{i}}$ and hence get $\mathbb{Z}_{p} \cong \mathcal{O}_{\mathfrak{p}_{i}}$. For every $i \in I$, we fix a uniformizer $\pi_{i}$ of $\mathcal{O}_{\mathfrak{p}_{i}}$ and let $\pi:=\prod_{i \in I} \pi_{i} \in \mathcal{O}_{p}$.

(3) For every $i \in I$, we use $\Delta_{i}$ to denote the torsion subgroup of $\mathcal{O}_{\mathfrak{p}_{i}}^{\times}$. Hence we have a decomposition of the multiplicative group $\mathcal{O}_{\mathfrak{p}_{i}}^{\times} \cong \Delta_{i} \times\left(1+\pi_{i} \mathcal{O}_{\mathfrak{p}_{i}}\right)$.

(4) Let $A_{F, f}$ (resp. $A_{F, f}^{(p)}$ ) denote the ring of finite adeles (resp. finite prime-to- $p$ adeles) of $F$.

(5) We use $\mathrm{Nm}_{F / \mathbb{Q}}: F^{\times} \rightarrow \mathbb{Q}^{\times}$to denote the norm map. For simplicity, the norm map $F_{p}^{\times} \rightarrow \mathbb{Q}_{p}^{\times}$will also be denoted by $\mathrm{Nm}_{F / \mathbb{Q}}$.

(6) Following [5, we define $\mathcal{N}:=\left\{|x|_{p} \mid x \in \overline{\mathbb{Q}}_{p}^{\times}\right\} \cap(0,1]=p^{\mathbb{Q}} \cap(0,1]$ and $\mathcal{N}^{\times}:=$ $\mathcal{N}\{1\}=p^{\mathbb{Q}} \cap(0,1)$.

(7) We use $\mathbb{A}^{1}$ (resp. $\mathbb{G}_{m}$ ) to denote the rigid analytification of the affine line (resp. affine line with zero removed).

(8) For $r=\left(r_{i}\right)_{i \in I} \in \mathcal{N}^{I}$ and $\alpha=\left(\alpha_{i}\right)_{i \in I} \in \mathcal{O}_{p}$, we define $B(\alpha, r)$ to be the $\mathbb{Q}_{p}$-polydisc such that $B(\alpha, r)\left(\mathbb{C}_{p}\right):=\left\{\left(x_{i}\right)_{i \in I} \in \mathbb{C}_{p}^{I}|| x_{i}-\left.i_{p}\left(\alpha_{i}\right)\right|_{p} \leqslant r_{i}\right.$ for all $\left.i \in I\right\}$, and $\mathbf{B}_{r}:=\prod_{\alpha \in \mathcal{O}_{p}} B(\alpha, r)$. If $r \in\left(\mathcal{N}^{\times}\right)^{I}$, we define $\mathbf{B}_{r}^{\times}:=\prod_{\alpha \in \mathcal{O}_{p}^{\times}} B(\alpha, r)$.

(9) Let $K$ be a complete field extension of $\mathbb{Q}_{p}$ and $X:=\operatorname{Sp}(A)$ be a $K$-affinoid space. By 5. Lemma 8.2(a)], there is a bijection $\iota: \mathcal{O}(X)^{\times} \cong \operatorname{Hom}_{\mathbb{Q}_{p} \text {-rigid space }}\left(X, \mathbb{G}_{m}\right)$. By 5, Proposition 8.3], for any continuous character $\chi: \mathcal{O}_{p}^{\times} \rightarrow A^{\times}$, there exists at least one $r \in\left(\mathcal{N}^{\times}\right)^{I}$ that satisfies the following:

There is a unique map of $K$-rigid spaces $\beta_{r}: \mathbf{B}_{r}^{\times} \times X \rightarrow \mathbb{G}_{m}$ such that for every $\alpha \in \mathcal{O}_{p}^{\times}$we have $\iota \circ \chi(\alpha)=\beta_{r}(\alpha,-)$, where $\chi(\alpha) \in A^{\times}=\mathcal{O}(X)^{\times}$and $\beta_{r}(\alpha,-)$ : $X \rightarrow \mathbb{G}_{m}$ is obtained by evaluating $\beta_{r}$ at $\alpha \in \mathbf{B}_{r}^{\times}\left(\mathbb{Q}_{p}\right)$.

We call $\beta_{r}$ the $r$-thickening of $\chi$.

(10) Let $\chi: \mathcal{O}_{p}^{\times} \rightarrow \mathbb{C}_{p}^{\times}$be a continuous character. For every $i \in I$, we denote by $\chi_{i}$ the $i$-component of $\chi$, i.e. $\chi_{i}$ is the composite $\mathcal{O}_{\mathfrak{p}_{i}}^{\times} \hookrightarrow \mathcal{O}_{p}^{\times} \stackrel{\chi}{\rightarrow} \mathbb{C}_{p}^{\times}$. We define the coordinates $T:=\left(T_{i}\right)_{i \in I}$ of $\chi$ by $T_{i}:=T_{\chi_{i}}=\chi_{i}\left(\exp \left(\pi_{i}\right)\right)-1$ for all $i \in I$. For $r=\left(r_{i}\right)_{i \in I} \in\left(\mathcal{N}^{\times}\right)^{I}$, the character $\chi$ admits the $r$-thickening if and only if $v_{p}\left(T_{i}\right)>\frac{p r_{i}}{p-1}$ for all $i \in I$.

(11) Let $\iota_{F}: \mathcal{O}_{F}^{\times} \hookrightarrow \mathcal{O}_{p}^{\times} \times \mathcal{O}_{p}^{\times}, x \mapsto\left(x, x^{2}\right)$ be an embedding. We always regard $\mathcal{O}_{F}^{\times}$ as a subgroup of $\mathcal{O}_{p}^{\times} \times \mathcal{O}_{p}^{\times}$by $\iota_{F}$. A weight is defined to be a continuous group homomorphism $\kappa=(n, \nu): \mathcal{O}_{p}^{\times} \times \mathcal{O}_{p}^{\times} \rightarrow A^{\times}$for some affinoid $\mathbb{Q}_{p}$-space $X=\operatorname{Sp}(A)$, such that $\operatorname{ker}(\kappa)$ contains a subgroup of $\mathcal{O}_{F}^{\times}$of finite index. For such a weight $\kappa$, we define $r(\kappa)$ to be the largest element $r$ in $\left(\mathcal{N}^{\times}\right)^{I}$ (with the obvious partial order), such that the continuous homomorphism $n: \mathcal{O}_{p}^{\times} \rightarrow \mathcal{O}(X)^{\times}$admits the $r$-thickening.

(12) Let $D / F$ be a totally definite quaternion algebra over $F$ with discriminant $\mathfrak{d}$. Assume that $(p, \mathfrak{d})=1$. Let $D_{f}:=D \otimes_{F} A_{F, f}$ and $D_{f}^{(p)}:=D \otimes_{F} A_{F, f}^{(p)}$. Fix a maximal order $\mathcal{O}_{D}$ of $D$. For each finite place $v$ of $F$ prime to $\mathfrak{d}$, we fix an isomorphism $\mathcal{O}_{D} \otimes_{\mathcal{O}_{F}} \mathcal{O}_{F, v} \cong \mathrm{M}_{2}\left(\mathcal{O}_{F, v}\right)$. In particular, we have an isomorphism $\mathcal{O}_{D} \otimes_{\mathcal{O}_{F}} \mathcal{O}_{p} \cong$ 
$\mathrm{M}_{2}\left(\mathcal{O}_{p}\right)$. For an ideal $\mathfrak{n}$ of $\mathcal{O}_{F}$ prime to $\mathfrak{d}$, we denote by $U_{0}^{D,(p)}(\mathfrak{n})$ and $U_{1}^{D,(p)}(\mathfrak{n})$ the subgroups of $\left(\mathcal{O}_{D} \otimes \hat{\mathbb{Z}}^{(p)}\right)^{\times}$consisting of matrices which are congruent to $\left[\begin{array}{ll}* & * \\ 0 & *\end{array}\right]$ and $\left[\begin{array}{ll}* & * \\ 0 & 1\end{array}\right] \bmod \mathfrak{n}$, respectively.

(13) For a $\mathbb{Q}_{p}$-Banach algebra $A$ with norm $|\cdot|$, we define a subring $A\{\{X\}\}$ of $A \llbracket X \rrbracket$ by

$$
A\{\{X\}\}:=\left\{\sum_{n \geqslant 0} c_{n} X^{n} \in A \llbracket X \rrbracket\left|\lim _{n \rightarrow \infty}\right| c_{n} \mid R^{n}=0, \text { for any } R \in \mathbb{R}_{>0}\right\} .
$$

\subsection{Subgroups of $\mathrm{GL}_{2}\left(F_{p}\right)$.}

(1) For a commutative ring $R$ with unit 1 , we define several subgroups of $\mathrm{GL}_{2}(R)$ by

$$
B(R):=\left[\begin{array}{cc}
R^{\times} & R \\
0 & R^{\times}
\end{array}\right], T(R):=\left[\begin{array}{cc}
R^{\times} & 0 \\
0 & R^{\times}
\end{array}\right], N(R):=\left[\begin{array}{cc}
1 & R \\
0 & 1
\end{array}\right] \text { and } D(R):=\left\{\left[\begin{array}{cc}
\alpha & 0 \\
0 & \alpha
\end{array}\right] \mid \alpha \in R^{\times}\right\} .
$$

For a subgroup $H$ of $R^{\times}$, we set

$$
T(H):=\left[\begin{array}{cc}
H^{\times} & 0 \\
0 & H^{\times}
\end{array}\right] \text {and } D(H):=\left\{\left[\begin{array}{cc}
\alpha & 0 \\
0 & \alpha
\end{array}\right] \mid \alpha \in H\right\} .
$$

(2) Fix $i \in I$ and a positive integer $t_{i}$. We define the following subgroups of $\operatorname{GL}_{2}\left(\mathcal{O}_{\mathfrak{p}_{i}}\right)$ :

$$
\operatorname{Iw}_{\pi_{i}^{t_{i}}}:=\left[\begin{array}{cc}
\mathcal{O}_{\mathfrak{p}_{i}}^{\times} & \mathcal{O}_{\mathfrak{p}_{i}} \\
\pi_{i}^{t_{i}} \mathcal{O}_{\mathfrak{p}_{i}} & \mathcal{O}_{\mathfrak{p}_{i}}^{\times}
\end{array}\right], \bar{N}\left(\pi_{i}^{t_{i}} \mathcal{O}_{\mathfrak{p}_{i}}\right):=\left[\begin{array}{cc}
1 & 0 \\
\pi_{i}^{t_{i}} \mathcal{O}_{\mathfrak{p}_{i}} & 1
\end{array}\right] \text { and } \bar{B}\left(\pi_{i}^{t_{i}} \mathcal{O}_{\mathfrak{p}_{i}}\right):=\left[\begin{array}{cc}
\mathcal{O}_{\mathfrak{p}_{i}}^{\times} & 0 \\
\pi_{i}^{t_{i}} \mathcal{O}_{\mathfrak{p}_{i}} & \mathcal{O}_{\mathfrak{p}_{i}}^{\times}
\end{array}\right]
$$

Given $t=\left(t_{i}\right)_{i \in I} \in \mathbb{N}^{I}$, we put $\mathrm{Iw}_{\pi^{t}}:=\prod_{i \in I} \operatorname{Iw}_{\pi_{i}^{t_{i}}}$ and similarly define $\bar{N}\left(\pi^{t} \mathcal{O}_{p}\right), \bar{B}\left(\pi^{t} \mathcal{O}_{p}\right)$.

Note that every $g \in \mathrm{Iw}_{\pi^{t}}$ can be represented by $\left(g_{i}\right)_{i \in I}$, with $g_{i} \in \mathrm{Iw}_{\pi_{i} m_{i}}$, and every $g_{i} \in \mathrm{Iw}_{\pi_{i}^{t_{i}}}$ can be viewed as an element in $\mathrm{Iw}_{\pi^{t}}$ whose $j$-component is the identity matrix for all $j \neq i$. All the groups we define above are profinite and considered as topological groups endowed with the profinite topology.

(3) For $i \in I$ and $t_{i} \in \mathbb{N}$, we will identify $\mathcal{O}_{\mathfrak{p}_{i}}$ with $\bar{N}\left(\pi_{i}^{t_{i}} \mathcal{O}_{\mathfrak{p}_{i}}\right)$ via the isomorphism $\bar{n}_{i}: \mathcal{O}_{\mathfrak{p}_{i}} \rightarrow \bar{N}\left(\pi_{i}^{t_{i}} \mathcal{O}_{\mathfrak{p}_{i}}\right), z \mapsto\left[\begin{array}{cc}1 & 0 \\ & \\ \pi_{i}^{t_{i}} z & 1\end{array}\right]$. For $t \in \mathbb{N}^{I}$, we will identify $\mathcal{O}_{p}$ with $\bar{N}\left(\pi^{t} \mathcal{O}_{p}\right)$ via the isomorphism $\bar{n}: \mathcal{O}_{p} \rightarrow \bar{N}\left(\pi^{t} \mathcal{O}_{p}\right), z \mapsto\left[\begin{array}{cc}1 & 0 \\ \pi^{t} z & 1\end{array}\right]$.

(4) For $i \in I$, we denote by $\operatorname{Iw}_{\pi_{i}, 1}:=\left[\begin{array}{cc}1+\pi_{i} \mathcal{O}_{\mathfrak{p}_{i}} & \mathcal{O}_{\mathfrak{p}_{i}} \\ \pi_{i} \mathcal{O}_{\mathfrak{p}_{i}} z & 1+\pi_{i} \mathcal{O}_{\mathfrak{p}_{i}}\end{array}\right]$ the the pro- $p$-subgroup of $\operatorname{Iw}_{\pi_{i}}$. Then the map $T\left(\Delta_{i}\right) \times \mathrm{Iw}_{\pi, 1} \rightarrow \mathrm{Iw}_{\pi},(t, g) \mapsto t g$ is a bijection. We put $\operatorname{Iw}_{\pi, 1}:=\prod_{i \in I} \operatorname{Iw}_{\pi_{i}, 1}$.

(5) The Iwasawa decomposition is the following bijection:

$$
N\left(\mathcal{O}_{p}\right) \times T\left(\mathcal{O}_{p}\right) \times \bar{N}\left(\pi^{t} \mathcal{O}_{p}\right) \rightarrow \operatorname{Iw}_{\pi^{t}},(N, T, \bar{N}) \mapsto N T \bar{N}
$$

(6) For $i \in I$ and $t_{i} \in \mathbb{N}$, we define an anti-involution $*$ on $\operatorname{Iw}_{\pi_{i}^{t_{i}}}$ by

$$
g=\left[\begin{array}{ll}
a & b \\
c & d
\end{array}\right] \in \mathrm{Iw}_{\pi_{i}^{t_{i}}} \mapsto g^{*}=\left[\begin{array}{cc}
a & c / \pi_{i}^{t_{i}} \\
\pi_{i}^{t_{i}} b & d
\end{array}\right] .
$$


Here "anti-involution" means that the map $g \mapsto g^{*}$ satisfies $\left(g^{*}\right)^{*}=g$ and $(g h)^{*}=$ $h^{*} g^{*}$ for all $g, h \in \mathrm{Iw}_{\pi_{i}} t_{i}$. We use similar formula to define anti-involutions on $\operatorname{Iw}_{\pi^{t}}$ for $t \in \mathbb{N}^{I}$.

(7) For $t=\left(t_{i}\right)_{i \in I} \in \mathbb{N}^{I}$, we set

$$
\mathbf{M}_{\pi^{t}}:=\left\{\gamma=\left(\gamma_{i}\right)_{i \in I} \in \mathbf{M}_{2}\left(\mathcal{O}_{p}\right) \mid \text { if } \gamma_{i}=\left[\begin{array}{cc}
a_{i} & b_{i} \\
c_{i} & d_{i}
\end{array}\right] \text { then } \operatorname{det}\left(\gamma_{i}\right) \neq 0, \pi_{i}^{t_{i}} \mid c_{i}, \pi_{i} \nmid d_{i}\right\} \text {. }
$$

Then $\mathbf{M}_{\pi^{t}}$ contains $\mathrm{Iw}_{\pi^{t}}$ and is a monoid under multiplication. The involution * can be extended to $\mathbf{M}_{\pi^{t}}$ by the same formula. For $i \in I$ and $t_{i} \in \mathbb{N}$, we define the monoid $\mathbf{M}_{\pi_{i}^{t_{i}}} \subset \mathrm{M}_{2}\left(\mathcal{O}_{\mathfrak{p}_{i}}\right)$ in a similar way.

3.3. Induced representations. Let $A$ be a topological ring in which $p$ is topologically nilpotent and $\kappa=(n, \nu): \mathcal{O}_{p}^{\times} \times \mathcal{O}_{p}^{\times} \rightarrow A^{\times}$be a continuous character.

Convention 3.3.1. Given the character $\kappa$ as above, we always extend $\nu$ to a continuous homomorphism $F_{p}^{\times} \rightarrow A^{\times}$by requiring $\nu\left(\pi_{i}\right)=1$ for all $i \in I$.

The character $\kappa$ induces a character $\kappa_{T}: T\left(\mathcal{O}_{p}\right) \rightarrow A^{\times},\left[\begin{array}{ll}a & 0 \\ 0 & d\end{array}\right] \mapsto n(d) \cdot \nu(a d)$. Since $T\left(\mathcal{O}_{p}\right)$ is a quotient of $B\left(\mathcal{O}_{p}\right)$ (resp. $\bar{B}\left(\pi \mathcal{O}_{p}\right)$ ), this character $\kappa_{T}$ extends to a character $\kappa_{B}$ (resp. $\kappa_{\bar{B}}$ ) of $B\left(\mathcal{O}_{p}\right)$ (resp. $\bar{B}\left(\pi \mathcal{O}_{p}\right)$ ).

Fix $t \in \mathbb{N}^{I}$. Consider the induced representation

$$
\operatorname{Ind}_{B\left(\mathcal{O}_{p}\right)}^{\mathrm{Iw}_{\pi^{t}}}\left(\kappa_{B}\right):=\left\{f \in \mathcal{C}\left(\operatorname{Iw}_{\pi^{t}}, A\right) \mid f(b g)=\kappa_{B}(b) f(g) \text { for all } b \in B\left(\mathcal{O}_{p}\right), g \in \mathrm{Iw}_{\pi^{t}}\right\} .
$$

The group $\operatorname{Iw}_{\pi^{t}}$ acts right on $\operatorname{Ind}_{B\left(\mathcal{O}_{p}\right)}^{\mathrm{Iw}_{\pi^{t}}}\left(\kappa_{B}\right)$ by $f \circ m(g)=f\left(g m^{*}\right)$. By Iwasawa decomposition, we obtain the following bijection

$$
\begin{aligned}
\operatorname{Ind}_{B\left(\mathcal{O}_{p}\right)}^{\mathrm{Iw}_{\pi^{t}}}\left(\kappa_{B}\right) & \rightarrow \mathcal{C}\left(\mathcal{O}_{p}, A\right), \\
f & \mapsto h(z):=f(\bar{n}(z))=f\left(\left[\begin{array}{cc}
1 & 0 \\
\pi^{t} z & 1
\end{array}\right]\right) .
\end{aligned}
$$

The right action of $\operatorname{Iw}_{\pi^{t}}$ on $\operatorname{Ind}_{B\left(\mathcal{O}_{p}\right)}^{\mathrm{Iw}_{\pi t}}\left(\kappa_{B}\right)$ induces a right action on $\mathcal{C}\left(\mathcal{O}_{p}, A\right)$, which can be written in the following explicit formula:

$$
h \circ m(z)=n(c z+d) \nu(a d-b c) h\left(\frac{a z+b}{c z+d}\right), \text { for } m=\left[\begin{array}{ll}
a & b \\
c & d
\end{array}\right] .
$$

Using the exact formula in 3.2 , we can extend this action to the monoid $\mathbf{M}_{\pi^{t}}$.

3.4. Space of $p$-adic automorphic forms. We will recall Buzzard's construction of overconvergent automorphic forms in [5].

Let $A$ be a $\mathbb{Q}_{p}$-affinoid algebra and $X:=\operatorname{Sp}(A)$ be the corresponding affinoid space. Let $\kappa=(n, \nu): \mathcal{O}_{p}^{\times} \times \mathcal{O}_{p}^{\times} \rightarrow A^{\times}$be a weight. We write $r(\kappa):=\left(p^{-m_{\kappa, i}}\right)_{i \in I}$. Fix $r=\left(r_{i}\right)_{i \in I}=$ $\left(p^{-m_{i}}\right)_{i \in I} \in \mathcal{N}^{I}$. Define $\mathcal{A}_{\kappa, r}$ to be the $\mathbb{Q}_{p}$-Banach algebra $\mathcal{O}\left(\mathbf{B}_{r} \times X\right)=\mathcal{O}_{\mathbf{B}_{r}} \hat{\otimes}_{\mathbb{Q}_{p}} A$. Since $\mathcal{O}_{p}$ is Zariski dense in $\mathbf{B}_{r}$, we obtain an embedding $\mathcal{A}_{\kappa, r} \hookrightarrow \mathcal{C}\left(\mathcal{O}_{p}, A\right)$ and hence can view $\mathcal{A}_{\kappa, r}$ as a subspace of $\mathcal{C}\left(\mathcal{O}_{p}, A\right)$ via this embedding.

Definition 3.4.1. We call $t \in \mathbb{N}^{I}$ that is $\operatorname{good}$ for $(\kappa, r)$ if $m_{i}+t_{i} \geqslant m_{\kappa, i}$ for all $i \in I$.

Fix $t \in \mathbb{N}^{I}$ that is good for $(\kappa, r)$. It is easy to check that the right action of $\mathbf{M}_{\pi^{t}}$ on $\mathcal{C}\left(\mathcal{O}_{p}, A\right)$ leaves the subspace $\mathcal{A}_{\kappa, r}$ stable, and hence 3.2$)$ defines a right action of the monoid $\mathbf{M}_{\pi^{t}}$ on $\mathcal{A}_{\kappa, r}$. 
Fix an open compact subgroup $K^{p} \subset D_{f}^{(p), \times}$ and $t \in \mathbb{N}^{I}$ which is good for $(\kappa, r)$. As in [5], we define the space of $r$-overconvergent automorphic forms of weight $\kappa$ and level $K^{p} \mathrm{Iw}_{\pi^{t}}$ to be the $A$-module

$$
S_{\kappa}^{D}\left(K^{p} \mathrm{Iw}_{\pi^{t}}, r\right)=\left\{\phi: D^{\times} \backslash D_{f}^{\times} / K^{p} \rightarrow \mathcal{A}_{\kappa, r} \mid \phi(g u)=\phi(g) \circ u \text {, for all } g \in D_{f}^{\times}, u \in \operatorname{Iw}_{\pi^{t}}\right\} .
$$

3.5. Hecke operators. Let $\kappa=(n, \nu): \mathcal{O}_{p}^{\times} \times \mathcal{O}_{p}^{\times} \rightarrow A^{\times}, r=\left(r_{i}\right)_{i \in I} \in \mathcal{N}^{I}$, and $t \in \mathbb{N}^{I}$ be the same as the previous section. Fix an open compact subgroup $K^{p}$ of $D_{f}^{(p), \times}$. In the rest of this paper, we will make the following convention on the choice of $K^{p}$.

Convention 3.5.1. $K^{p}$ is of the form $U_{0}^{(p)}(\mathfrak{n})$ or $U_{1}^{(p)}(\mathfrak{n})$ for some ideal $\mathfrak{n}$ of $\mathcal{O}_{F}$ prime to $p \mathfrak{d}$.

Let $K=K^{p} \mathrm{Iw}_{\pi^{t}}$ be the open compact subgroup of $D_{f}^{\times}$. Now we recall the definition of Hecke operators on the space $S_{\kappa}^{D}\left(K^{p} \mathrm{Iw}_{\pi^{t}}, r\right)$.

Let $v$ be a finite place of $F$ where $D$ splits and fix a uniformizer $\pi_{v}$ of $F_{v}$. In particular, when $v=i \in I$ is over $p$, we choose $\pi_{v}=\pi_{i}$ as before. Let $\eta_{v}=\left[\begin{array}{cc}\pi_{v} & 0 \\ 0 & 1\end{array}\right] \in \operatorname{GL}_{2}\left(F_{v}\right)$ which is viewed as an element in $D_{f}^{\times}$whose components away from $v$ are the identity element. We fix a double coset decomposition

$$
K \eta_{v} K=\bigsqcup_{j} K x_{j}
$$

with $x_{j} \in D_{f}^{\times}$. We use $x_{j, p}$ to denote the $v$-component of $x_{j}$. The Hecke operator $U_{v}$ on $S_{\kappa}^{D}\left(K^{p} \operatorname{Iw}_{\pi^{t}}, r\right)$ is defined by

$$
U_{v}(\phi):=\left.\sum_{j} \phi\right|_{x_{j}}
$$

where $\left.\phi\right|_{x_{j}}(x):=\phi\left(x x_{j}^{-1}\right) \circ x_{j, p}$ for $\phi \in S_{\kappa}^{D}\left(K^{p} \operatorname{Iw}_{\pi^{t}}, r\right)$.

When $v=i \in I$, the operator $U_{i}$ depends on the choice of the uniformizer $\pi_{i}$, and we will write $U_{\pi_{i}}$ for $U_{i}$. For later computations, we give a more explicit expression of the $U_{\pi_{i}}$ operator. We fix a double coset decomposition

$$
\mathrm{Iw}_{\pi^{t}} \eta_{i} \mathrm{Iw}_{\pi^{t}}=\bigsqcup_{j=0}^{p-1} \mathrm{Iw}_{\pi^{t}} v_{i, j}
$$

with $v_{i, j}=\left[\begin{array}{cc}\pi_{i} & 0 \\ j \pi_{i}^{t_{i}} & 1\end{array}\right] \in \mathbf{M}_{\pi_{i}^{t_{i}}} \subset \mathbf{M}_{\pi^{t}}$ for $j=0, \ldots, p-1$. The $U_{\pi_{i}}$-operator can be described by

$$
U_{\pi_{i}}(\phi)=\left.\sum_{j} \phi\right|_{v_{i, j}} \text { with }\left.\phi\right|_{v_{i, j}}(x)=\phi\left(x v_{i, j}^{-1}\right) \circ v_{i, j} .
$$

When $v$ is prime to $\mathfrak{n} p$, we view $\pi_{v}$ as a central element in $D_{f}^{\times}$. Choose a double coset decomposition

$$
K \pi_{v} K=\bigsqcup_{j} K y_{j}
$$

with $y_{j} \in D_{f}^{\times}$. The Hecke operator $S_{v}$ on $S_{\kappa}^{D}\left(K^{p} \mathrm{Iw}_{\pi^{t}}, r\right)$ is defined by

$$
S_{v}(\phi)=\left.\sum_{j} \phi\right|_{y_{j}} .
$$


The Hecke operators $U_{v}$ 's and $S_{v}$ 's for all possible $v$ 's commute with each other. In particular, we define $U_{\pi}=\prod_{i \in I} U_{\pi_{i}}$.

By 5, Lemma 12.2], the operator $U_{\pi}$ acting on $S_{\kappa}^{D}\left(K^{p} \mathrm{Iw}_{\pi^{t}}, r\right)$ is compact. Thus, its characteristic power series is well-defined by

$$
\operatorname{Char}\left(U_{\pi} ; S_{\kappa}^{D}\left(K^{p} \mathrm{Iw}_{\pi^{t}}, r\right)\right):=\operatorname{det}\left(\mathrm{I}-\left.X U_{\pi}\right|_{S_{\kappa}^{D}\left(K^{p} \operatorname{Iw}_{\pi^{t}}, r\right)}\right) \in A\{\{X\}\} .
$$

We make two remarks on the characteristic power series $\operatorname{Char}\left(U_{\pi} ; S_{\kappa}^{D}\left(K^{p} \mathrm{Iw}_{\pi^{t}}, r\right)\right)$.

Remark 3.5.2. (1) By 5, Lemma 13.1], Char $\left(U_{\pi} ; S_{\kappa}^{D}\left(K^{p} \mathrm{Iw}_{\pi^{t}}, r\right)\right)$ is independent of $r$ (as long as $t$ is good for the pair $(\kappa, r)$ ).

(2) By [5, Proposition 11.1], there is a canonical Hecke equivariant isomorphism

$$
S_{\kappa}^{D}\left(K^{p} \operatorname{Iw}_{\pi^{t}}, r\right) \cong S_{\kappa}^{D}\left(K^{p} \mathrm{Iw}_{\pi}, r^{\prime}\right)
$$

for some explicit $r^{\prime} \in\left(\mathcal{N}^{\times}\right)^{I}$. It is a well-known phenomenon that the characteristic power series does not see the higher $\mathrm{Iw}_{\pi^{t}}$-structure. For this reason, we can and will only work over the space $S_{\kappa}^{D}\left(K^{p} \mathrm{Iw}_{\pi}, r\right)$, i.e. $t=(1, \ldots, 1)$.

3.6. The eigenvariety datum for $D$. In this section, we recall the construction of the spectral varieties and eigenvarieties associated to $D$ as constructed in [5, Part III]. We will follow [12, §4] to define an eigenvariety datum as we will use Hansen's interpolation theorem to translate our results to Hilbert modular eigenvarieties (see $\$ 6$ below).

We start with the definition of the weight space. As in 1], the weight space $\mathcal{W}$ is defined to be the rigid analytic space over $\mathbb{Q}_{p}$ associated to the complete group algebra $\mathbb{Z}_{p} \llbracket \mathcal{O}_{p}^{\times} \times \mathbb{Z}_{p}^{\times} \rrbracket$. A closed point of $\mathcal{W}$ is a continuous character $\chi=(\nu, \mu): \mathcal{O}_{p}^{\times} \times \mathbb{Z}_{p}^{\times} \rightarrow \mathbb{C}_{p}^{\times}$.

Remark 3.6.1. It will be helpful to compare the weight space constructed in [5] with the weight space constructed above.

First we recall its construction in $[5]$. Let $G \subset \mathcal{O}_{F}^{\times}$be a subgroup of finite index. Recall that we regard $G$ as a subgroup of $\mathcal{O}_{p}^{\times} \times \mathcal{O}_{p}^{\times}$via the embedding $\iota_{F}: \mathcal{O}_{F}^{\times} \rightarrow \mathcal{O}_{p}^{\times} \times \mathcal{O}_{p}^{\times}$, $x \mapsto\left(x, x^{2}\right)$. We denote by $\Gamma_{G}$ the quotient of $\mathcal{O}_{p}^{\times} \times \mathcal{O}_{p}^{\times}$by the closure of $G$. By 5 , Lemma 8.2], the functor which sends a $\mathbb{Q}_{p}$-rigid space $U$ to the group of continuous group homomorphism $\Gamma_{G} \rightarrow \mathcal{O}(U)^{\times}$is represented by a $\mathbb{Q}_{p}$-rigid space $\mathcal{X}_{\Gamma_{G}}$ (actually $\mathcal{X}_{\Gamma_{G}}$ is isomorphic to the product of an open unit polydisc and a finite rigid space over $\mathbb{Q}_{p}$ ). The weight space $\mathcal{W}^{\text {full }}$ is the direct $\operatorname{limit} \underset{G}{\lim _{G}} \mathcal{X}_{\Gamma_{G}}$ as $G$ varies over the set of subgroups of finite index of $\mathcal{O}_{F}^{\times}$. If $G_{1} \subset G_{2} \subset \mathcal{O}_{F}^{\times}$are two subgroups of $\mathcal{O}_{F}^{\times}$of finite indices, the natural homomorphism $\Gamma_{G_{1}} \rightarrow \Gamma_{G_{2}}$ is surjective with finite kernel, and hence the corresponding map $\mathcal{X}_{\Gamma_{G_{2}}} \rightarrow \mathcal{X}_{\Gamma_{G_{1}}}$ is a closed immersion and geometrically identifies $\mathcal{X}_{\Gamma_{G_{2}}}$ with a union of components of $\mathcal{X}_{\Gamma_{G_{1}}}$. Therefore, $\left\{\mathcal{X}_{\Gamma_{G}} \mid G\right.$ is a subgroup of $\mathcal{O}_{F}^{\times}$of finite index $\}$forms an admissible cover of the weight space $\mathcal{W}$. It follows that the dimension of $\mathcal{W}^{\text {full }}$ is $g+1+\delta$, where $\delta$ is the Leopoldt defect for $(F, p)$.

Now let's explain the relation between the two weight spaces $\mathcal{W}$ and $\mathcal{W}^{\text {full }}$. Define a continuous homomorphism $\phi_{\eta}: \mathcal{O}_{p}^{\times} \times \mathcal{O}_{p}^{\times} \rightarrow \mathcal{O}_{p}^{\times} \times \mathbb{Z}_{p}^{\times},(\alpha, \beta) \mapsto\left(\alpha^{-2} \beta, \operatorname{Nm}_{F / \mathbb{Q}}(\alpha)\right)$. The kernel $\operatorname{ker}\left(\phi_{\eta}\right)$ contains the group $G=\mathcal{O}_{F}^{\times,+}$and hence $\phi_{\eta}$ induces a map $\eta: \mathcal{W} \rightarrow \mathcal{X}_{\Gamma_{G}}$ of rigid analytic spaces over $\mathbb{Q}_{p}$ and hence a map $\eta: \mathcal{W} \rightarrow \mathcal{W}^{\text {full }}$. Since $\operatorname{dim} \mathcal{W}=g+1$, if the Leopoldt's conjecture holds for $(F, p)$, the map $\eta: \mathcal{W} \rightarrow \mathcal{W}^{\text {full }}$ would be an immersion which identifies $\mathcal{W}$ with a set of connected components of $\mathcal{W}^{\text {full }}$.

There are two reasons why we use the weight space $\mathcal{W}$ instead of $\mathcal{W}^{\text {full: }}$ first, we do not even know the dimension of the weight space $\mathcal{W}^{\text {full }}$ without assuming the Leopoldt's conjecture for $(F, p)$ and it is rather complicated to describe the connected components of 
$\mathcal{W}^{\text {full }}$ and classical weights on every component; second, as an application we will use $p$-adic Jacquet-Langlands correspondence to translate our results to Hilbert modular eigenvarieties as constructed in [1], and the weight space $\mathcal{W}$ is the one considered there.

Since the action of the monoid $\mathbf{M}_{\pi}$ on the spaces $\mathcal{A}_{\kappa, r}$ depends on the characters of $\mathcal{O}_{p}^{\times} \times \mathcal{O}_{p}^{\times}$, it is useful to have an explicit expression of the map $\mathcal{W} \rightarrow \mathcal{W}^{\text {full }}$ in term of characters. Let $A$ be an affinoid $\mathbb{Q}_{p}$-algebra and $\chi=(\nu, \mu): \mathcal{O}_{p}^{\times} \times \mathbb{Z}_{p}^{\times} \rightarrow A^{\times}$be a continuous character. Then the composite $\kappa=\chi \circ \phi_{\eta}: \mathcal{O}_{p}^{\times} \times \mathcal{O}_{p}^{\times} \rightarrow A^{\times}$is of the form $\kappa=(n, \nu)$, where $n: \mathcal{O}_{p}^{\times} \rightarrow A^{\times}$is the character defined by $\alpha \mapsto \nu(\alpha)^{-2} \cdot\left(\mu \circ \mathrm{Nm}_{F / \mathbb{Q}}(\alpha)\right)$. The character $\kappa$ is called the character of $\mathcal{O}_{p}^{\times} \times \mathcal{O}_{p}^{\times}$associated to $\chi$.

Convention 3.6.2. In the rest of this paper, a weight $\kappa: \mathcal{O}_{p}^{\times} \times \mathcal{O}_{p}^{\times} \rightarrow A^{\times}$is always the character associated to some character $\chi$ of $\mathcal{O}_{p}^{\times} \times \mathbb{Z}_{p}^{\times}$as constructed above.

We fix an open compact subgroup $K^{p}$ of $D_{f}^{(p), \times}$ as before. For an affinoid subdomain $U=\operatorname{Sp}(A) \subset \mathcal{W}$, we denote by $\chi_{U}: \mathcal{O}_{p}^{\times} \times \mathbb{Z}_{p}^{\times} \rightarrow A^{\times}$the universal character and $\kappa_{U}$ : $\mathcal{O}_{p}^{\times} \times \mathcal{O}_{p}^{\times} \rightarrow A^{\times}$the character associated to $\chi_{U}$. For simplicity, we use $f_{U}(X)$ to denote $\operatorname{Char}\left(U_{\pi}, S_{\kappa_{U}}^{D}\left(K^{p} \mathrm{Iw}_{\pi}, r\left(\kappa_{U}\right)\right)\right) \in A\{\{X\}\}$.

The spectral variety wt $: \mathcal{Z}_{D} \rightarrow \mathcal{W}$ is defined to be the Fredholm hypersurface of $\mathcal{W} \times \mathbb{A}^{1}$ associated to the compact operator $U_{\pi}$. More precisely, for every affinoid $U$ of $\mathcal{W}, \mathrm{wt}^{-1}(U) \subset U \times \mathbb{A}^{1}$ is the zero locus of the characteristic power series $f_{U}(X)$. We use wt $: \mathcal{Z}_{D} \rightarrow \mathcal{W}\left(\right.$ resp. $\left.a_{\pi}^{-1}: \mathcal{Z}_{D} \rightarrow \mathbb{A}^{1}\right)$ to denote the first (resp. second) projection.

Definition 3.6.3. An affinoid open subdomain $Y \subset \mathcal{Z}_{D}$ is called slope adapted if there exist $h \in \mathbb{Q}$ and an affinoid $U=\operatorname{Sp}(A) \subset \mathcal{W}$, such that $Y=A\left\langle p^{h} X\right\rangle /\left(f_{U}(X)\right)$ is an affinoid open subset of $\mathcal{Z}_{D}$ and the natural map $Y \rightarrow U$ is finite and flat.

For such an slope adapted affinoid $Y$, the characteristic power series $f_{U}(X)$ admits a slope $\leqslant h$-factorization $f_{U}(X)=Q(X) \cdot R(X)$ and $\mathcal{O}(Y) \cong A[X] /(Q(X))$. More precisely, we have the following characterization of $Q(X)$ and $R(X)$ :

- $Q(X)$ is a polynomial whose leading coefficient is a unit and the slopes of the Newton polygon of $Q(X)$ are all $\leqslant h$; and

- $R(X)$ is an entire power series and the slopes of the Newton polygon of $R(X)$ are all $>h$.

Moreover, it follows from 5. Theorem 3.3] that the space $S_{\kappa_{U}}^{D}\left(K^{p} \operatorname{Iw}_{\pi}, r\left(\kappa_{U}\right)\right)$ admits a slope $\leqslant h$-decomposition $S_{\kappa_{U}}^{D}\left(K^{p} \operatorname{Iw}_{\pi}, r\left(\kappa_{U}\right)\right)=S_{\kappa_{U}}^{D, \leqslant h} \oplus S_{\kappa_{U}}^{D,>h}$ in the sense of [12, Definition 2.3.1]. In particular, $S_{\kappa_{U}}^{D, \leqslant h}$ is an $\mathcal{O}(Y) \cong A[X] /(Q(X))$-module via the map $X \mapsto U_{\pi}^{-1}$. Recall that by [12, Proposition 4.1.4], the collection of slope adapted affinoids forms an admissible cover of $\mathcal{Z}_{D}$. Hence the association $Y \mapsto S_{\kappa_{U}}^{D, \leqslant h}$ defines a coherent sheaf $\mathcal{M}_{D}$ on $\mathcal{Z}_{D}$.

Let $\mathbf{T}$ be the Hecke algebra over $\mathbb{Q}_{p}$ generated by the Hecke operators $U_{v}$ 's and $S_{v}$ 's for all finite places $v$ of $F$ not dividing $p \mathfrak{n} \mathfrak{d}$, and all the $U_{\pi_{i}}$ 's for all $i \in I$ defined in $\$ 3.5$ and $\psi: \mathbf{T} \rightarrow \operatorname{End}_{\mathcal{Z}_{D}}\left(\mathcal{M}_{D}\right)$ be the natural homomorphism of $\mathbb{Q}_{p}$-algebras. The tuple $\mathfrak{D}=\left(\mathcal{W}, \mathcal{Z}_{D}, \mathcal{M}_{D}, \mathbf{T}, \psi\right)$ is an eigenvariety datum in the sense of [12, Definition 4.2.1]. We use $\mathcal{X}_{D}$ to denote the associated eigenvariety together with the finite morphism $\pi: \mathcal{X}_{D} \rightarrow \mathcal{Z}_{D}$ and a morphism $w: \mathcal{X}_{D} \rightarrow \mathcal{W}$. It follows from [12, Theorem 4.2.2] that every point $x$ of $\mathcal{X}_{D}$ lying over $z \in \mathcal{Z}_{D}$ corresponds to a generalized eigenspace for the action of $\mathbf{T}$ on $\mathcal{M}_{D}(z)$. In particular, we use $a_{i}(x)$ to denote the eigenvalue of the $U_{\pi_{i}}$-operator for all $i \in I$.

Let $\mathcal{W}^{*}$ be the rigid analytic space associated to $\mathbb{Z}_{p} \llbracket \mathcal{O}_{p}^{\times} \rrbracket$. The homomorphism $\phi_{\rho}$ : $\mathcal{O}_{p}^{\times} \rightarrow \mathcal{O}_{p}^{\times} \times \mathbb{Z}_{p}^{\times}, \alpha \mapsto\left(\alpha^{-2}, \mathrm{Nm}_{F / \mathbb{Q}}(\alpha)\right)$ induces a map of rigid spaces $\rho: \mathcal{W} \rightarrow \mathcal{W}^{*}$. Explicitly $\rho$ maps a weight $(\nu, \mu)$ of $\mathcal{O}_{p}^{\times} \times \mathbb{Z}_{p}^{\times}$to the character $n$ of $\mathcal{O}_{p}^{\times}$defined above. Each closed point of $\mathcal{W}^{*}$ corresponds to a continuous character $n: \mathcal{O}_{p}^{\times} \rightarrow \mathbb{C}_{p}^{\times}$. The coordinates 
$T_{i}^{*}$ for $i \in I$ of $n$ defined in $\$ 3.1$ form a set of parameters on $\mathcal{W}^{*}$. Let $T_{i}:=\phi_{\rho}\left(T_{i}^{*}\right)$ and $T:=[1, \exp (p)]-1 \in \mathbb{Z}_{p} \llbracket \mathcal{O}_{p} \times \mathbb{Z}_{p} \rrbracket$. The set $\left\{\left(T_{i}\right)_{i \in I}, T\right\}$ forms a full set of parameters on the weight space $\mathcal{W}$. Let $J$ be a nonempty subset of $I$ and $r_{J}:=\left(r_{j}\right)_{j \in J} \in(0,1)^{J}$. We denote by $\mathcal{W}^{>r_{J}}$ the subspace of $\mathcal{W}$ where $\left|T_{j}\right|_{p}>r_{j}$ for all $j \in J$. Set $\mathcal{Z}_{D}^{>r_{J}}:=\mathrm{wt}^{-1}\left(\mathcal{W}^{>r_{J}}\right)$ and $\mathcal{X}_{D}^{>r_{J}}:=w^{-1}\left(\mathcal{W}^{>r_{J}}\right)$.

Remark 3.6.4. We can use a similar trick as in [20, Remark 2.14] to replace the open compact subgroup $K^{p}$ be a sufficiently small one. For an ideal $\mathfrak{n}^{\prime}$ of $\mathcal{O}_{F}$ prime to $p \delta_{D}$, let $K^{\prime p}=K^{p} \cap U_{0}^{(p)}\left(\mathfrak{n}^{\prime}\right)$ or $K^{p} \cap U_{1}^{(p)}\left(\mathfrak{n}^{\prime}\right)$. Then the spectral variety (resp. eigenvariety) for $K^{p}$ is a union of connected components of the spectral variety (resp. eigenvariety) for $K^{\prime p}$. Hence it suffices to prove the theorem for sufficiently small $K^{p}$.

3.7. Integral model of the space of $p$-adic automorphic forms. Let $A$ be a topological ring in which $p$ is topologically nilpotent and $\chi=(\nu, \mu): \mathcal{O}_{p}^{\times} \times \mathbb{Z}_{p}^{\times} \rightarrow A^{\times}$be a continuous character. Let $\kappa=(n, \nu): \mathcal{O}_{p}^{\times} \times \mathcal{O}_{p}^{\times} \rightarrow A^{\times}$be the associated character of $\mathcal{O}_{p}^{\times} \times \mathcal{O}_{p}^{\times}$. Recall that from $\kappa$, we can define a character $\kappa_{T}\left(\right.$ resp. $\left.\kappa_{B}, \kappa_{\bar{B}}\right)$ of $T\left(\mathcal{O}_{p}\right)\left(\operatorname{resp} . B\left(\mathcal{O}_{p}\right), \bar{B}\left(\pi \mathcal{O}_{p}\right)\right)$ as in $\$ 3.3$. For any $i \in I$, we use $\kappa_{i}=\left(n_{i}, \nu_{i}\right): \mathcal{O}_{\mathfrak{p}_{i}}^{\times} \times \mathcal{O}_{\mathfrak{p}_{i}}^{\times} \rightarrow A^{\times}$to denote the $i$-component of $\kappa$. We extend the character $\nu$ to a character $\nu: F_{p}^{\times} \rightarrow A^{\times}$as before.

Similar with [20, §2.7], we make the following definition.

Definition 3.7.1. We define the space of integral $p$-adic automorphic forms for $D$ to be

$$
\begin{aligned}
S_{\kappa, I}^{D}\left(K^{p}, A\right) & :=\left\{\phi: D^{\times} \backslash D_{f}^{\times} / K^{p} \rightarrow \operatorname{Ind}_{B\left(\mathcal{O}_{p}\right)}^{\mathrm{Iw}_{\pi}}(\kappa) \mid \phi(x u)=\phi(x) \circ u, \text { for all } x \in D_{f}^{\times}, u \in \mathrm{Iw}_{\pi}\right\} \\
& =\left\{\phi: D^{\times} \backslash D_{f}^{\times} / K^{p} \rightarrow \mathcal{C}\left(\mathcal{O}_{p}, A\right) \mid \phi(x u)=\phi(x) \circ u, \text { for all } x \in D_{f}^{\times}, u \in \mathrm{Iw}_{\pi}\right\} .
\end{aligned}
$$

Remark 3.7.2. For any $t \in \mathbb{N}^{I}$, we define the space of integral $p$-adic automorphic forms of level $K^{p} \mathrm{Iw}_{\pi^{t}}$ by

$S_{\kappa, I}^{D}\left(K^{p} \mathrm{Iw}_{\pi^{t}}, A\right):=\left\{\phi: D^{\times} \backslash D_{f}^{\times} / K^{p} \rightarrow \mathcal{C}\left(\mathcal{O}_{p}, A\right) \mid \phi(x u)=\phi(x) \circ u\right.$, for all $\left.x \in D_{f}^{\times}, u \in \mathrm{Iw}_{\pi^{t}}\right\}$.

This definition gives no generalization of Definition 3.7.1. In fact, for $t \in \mathbb{N}^{I}$ and $s \in$ $\mathbb{Z}_{\geqslant 0}^{I}$, we have an Hecke equivariant isomorphism between the spaces $S_{\kappa, I}^{D}\left(K^{p} \operatorname{Iw}_{\pi^{t}}, A\right)$ and $S_{\kappa, I}^{D}\left(K^{p} \mathrm{Iw}_{\pi^{t+s}}, A\right)$. This fact is well known to experts. For completeness, we give a sketch of the construction of the isomorphisms and refer [5, §11], especially Proposition 11.1 for more details.

For $\phi \in S_{\kappa, I}^{D}\left(K^{p} \operatorname{Iw}_{\pi^{t}}, A\right)$, we define $\psi: D^{\times} \backslash D_{f}^{\times} / K^{p} \rightarrow \mathcal{C}\left(\mathcal{O}_{p}, A\right)$ by

$$
\psi(x)=\phi\left(x\left[\begin{array}{cc}
\pi^{-s} & 0 \\
0 & 1
\end{array}\right]\right) \circ\left[\begin{array}{cc}
\pi^{s} & 0 \\
0 & 1
\end{array}\right] .
$$

One can check $\psi \in S_{\kappa, I}^{D}\left(K^{p} \operatorname{Iw}_{\pi^{t+s}}, A\right)$ and we obtain a map $\iota_{1}: S_{\kappa, I}^{D}\left(K^{p} \operatorname{Iw}_{\pi^{t}}, A\right) \rightarrow$ $S_{\kappa, I}^{D}\left(K^{p} \operatorname{Iw}_{\pi^{t+s}}, A\right)$.

Conversely, given $\psi \in S_{\kappa, I}^{D}\left(K^{p} \operatorname{Iw}_{\pi^{t+s}}, A\right)$, we define $\phi: D^{\times} \backslash D_{f}^{\times} / K^{p} \rightarrow \mathcal{C}\left(\mathcal{O}_{p}, A\right)$ by

$$
\phi(x)\left(z^{\prime}\right):=\psi\left(x\left[\begin{array}{cc}
\pi^{s} & \alpha \\
0 & 1
\end{array}\right]\right)(z) \text {, with } z^{\prime}=\pi^{s} z+\alpha \text { for some } z \text { and } \alpha \in \mathcal{O}_{p} .
$$

One can verify that this definition is independent of the expression of $z^{\prime}=\pi^{s} z+\alpha \in \mathcal{O}_{p}$ and $\phi \in S_{\kappa, I}^{D}\left(K^{p} \mathrm{Iw}_{\pi^{t}}, A\right)$. So we obtain another map $\iota_{2}: S_{\kappa, I}^{D}\left(K^{p} \mathrm{Iw}_{\pi^{t+s}}, A\right) \rightarrow S_{\kappa, I}^{D}\left(K^{p} \operatorname{Iw}_{\pi^{t}}, A\right)$. The two maps $\iota_{1}$ and $\iota_{2}$ establish the desired Hecke equivariant isomorphism.

For a better interpretation of Definition 3.7 .8 below, it is useful to explain the relation between the space of integral $p$-adic automorphic forms for $D$ and the completed homology 
group of the Shimura varieties associated to the algebraic group $\operatorname{Res}_{F / \mathbb{Q}} D^{\times}$. Recall the completed homology group (of degree 0) is defined as

$$
\tilde{\mathrm{H}}_{0}:={\underset{K}{K_{p}}}_{\lim _{0}} \mathrm{H}_{0}\left(D^{\times} \backslash D_{f}^{\times} / K^{p} K_{p}, \mathbb{Z}_{p}\right)=\underset{K_{p}}{\lim } \mathbb{Z}_{p}\left[D^{\times} \backslash D_{f}^{\times} / K^{p} K_{p}\right]
$$

where in the inverse limit, $K_{p}$ ranges over all open compact subgroups of $\mathrm{GL}_{2}\left(\mathcal{O}_{p}\right)$. For each $K_{p}$, the group $\mathrm{H}_{0}\left(D^{\times} \backslash D_{f}^{\times} / K^{p} K_{p}, \mathbb{Z}_{p}\right)$ is endowed with the $p$-adic topology and $\tilde{\mathrm{H}}_{0}$ is endowed with the inverse limit topology. The complete homology group $\tilde{\mathrm{H}}_{0}$ carries a natural right action of $\mathrm{GL}_{2}\left(F_{p}\right)$ induced by the right translation of $\mathrm{GL}_{2}\left(F_{p}\right)$ on $D_{f}^{\times}$. It follows from 11, Theorem 2.2] that $\tilde{\mathrm{H}}_{0}$ is a finitely generated $\mathbb{Z}_{p} \llbracket K_{p} \rrbracket$-module for any (nonempty) open compact subgroup $K_{p}$ of $\mathrm{GL}_{2}\left(F_{p}\right)$.

Proposition 3.7.3. Assume that $A$ is linearly topologized, i.e. $0 \in A$ has a fundamental system of neighborhoods consisting of ideals. There is a natural isomorphism of A-modules:

$$
S_{\kappa, I}^{D}\left(K^{p}, A\right) \rightarrow \operatorname{Hom}_{c t s, \bar{B}\left(\pi \mathcal{O}_{p}\right)}\left(\tilde{\mathrm{H}}_{0}, A\left(\kappa_{\bar{B}}\right)\right) .
$$

Proof. This result is well known to experts. We only give a sketch of the construction of the isomorphism here.

By definition, the induced representation $\operatorname{Ind}_{B\left(\mathcal{O}_{p}\right)}^{\mathrm{Iw}_{\pi}}(\kappa)$ is a subspace of $\mathcal{C}\left(\operatorname{Iw}_{\pi}, A\right)$. The right action of $\operatorname{Iw}_{\pi}$ on $\operatorname{Ind}_{B\left(\mathcal{O}_{p}\right)}^{\mathrm{Iw}_{\pi}}(\kappa)$ can be extended to $\mathcal{C}\left(\operatorname{Iw}_{\pi}, A\right)$ by the same formula: $f \circ u(g)=f\left(g u^{*}\right)$. It is worthwhile to remark here that the action of the monoid $\mathbf{M}_{1}$ cannot be extended to $\mathcal{C}\left(\mathrm{Iw}_{\pi}, A\right)$.

Given a map $\phi: D^{\times} \backslash D_{f}^{\times} / K^{p} \rightarrow \mathcal{C}\left(\operatorname{Iw}_{\pi}, A\right)$, with the property that $\phi(x u)=\phi(x) \circ u$, for all $x \in D_{f}^{\times}$and $u \in \operatorname{Iw}_{\pi}$. We define a map $\tilde{f}: D^{\times} \backslash D_{f}^{\times} / K^{p} \rightarrow A$ by $\tilde{f}(x)=\phi(x)\left(I_{2}\right)$, where $I_{2} \in \mathrm{Iw}_{\pi}$ is the identity matrix. Since $\phi(x) \in \mathcal{C}\left(\mathrm{Iw}_{\pi}, A\right)$, for all $x \in D_{f}^{\times}, \tilde{f}$ induces a continuous map $f: \tilde{\mathrm{H}}_{0} \rightarrow A$. Moreover, when $\phi \in S_{\kappa, I}^{D}\left(K^{p}\right)$, it is straightforward to check that $f \in \operatorname{Hom}_{c t s, \bar{B}\left(\pi \mathcal{O}_{p}\right)}\left(\tilde{\mathrm{H}}_{0}, A\left(\kappa_{\bar{B}}\right)\right)$.

Conversely, given a continuous map $f: \tilde{\mathrm{H}}_{0} \rightarrow A$, we view $f$ as a map $D^{\times} \backslash D_{f}^{\times} / K^{p} \rightarrow A$, such that $f \bmod I$ is locally constant on cosets of $\mathrm{GL}_{2}\left(F_{p}\right)$ for all nonempty open ideals $I$ of $A$ as $A$ is linearly topologized. Define $\phi: D^{\times} \backslash D_{f}^{\times} / K^{p} \rightarrow \mathcal{C}\left(\operatorname{Iw}_{\pi}, A\right)$ by $\phi(x)(g)=f\left(x g^{*}\right)$, for $x \in D_{f}^{\times}, g \in \mathrm{Iw}_{\pi}$. It is easy to check that $\phi(x u)=\phi(x) \circ u$, for $x \in D_{f}^{\times}$and $u \in \mathrm{Iw}_{\pi}$, and if $f \in \operatorname{Hom}_{c t s, \bar{B}\left(\pi \mathcal{O}_{p}\right)}\left(\tilde{\mathrm{H}}_{0}, A\left(\kappa_{\bar{B}}\right)\right)$, then $\phi(x) \in \operatorname{Ind}_{B\left(\mathcal{O}_{p}\right)}^{\mathrm{Iw}_{\pi}}(\kappa)$, for all $x \in D_{f}^{\times}$.

Remark 3.7.4. Conceptually, Proposition 3.7 .3 follows from the tautological isomorphism

$$
\left.S_{\kappa, I}^{D}\left(K^{p}, A\right)=\operatorname{Hom}_{\mathrm{Iw}_{p}}\left(D^{\times} \backslash D_{f}^{\times} / K^{p}, \operatorname{Ind}_{B\left(\mathcal{O}_{p}\right)}^{\mathrm{Iw}_{\pi}}(\kappa)\right) \cong \operatorname{Hom}_{\mathbb{Z}_{p} \llbracket \operatorname{Iw}_{\pi} \rrbracket}\left(\tilde{H}_{0}, \operatorname{Ind}_{B\left(\mathcal{O}_{p}\right)}^{\mathrm{Iw}_{\pi}}(\kappa)\right)\right)
$$

and Frobenius reciprocity. However, the induced representation $\operatorname{Ind}_{B\left(\mathcal{O}_{p}\right)}^{\mathrm{Iw}_{\pi}}(\kappa)$ is slightly different from the standard definition. This is why we give a concrete proof here.

Inspired by Proposition 3.7.3, if we replace the group $\bar{B}\left(\pi \mathcal{O}_{p}\right)$ by $\bar{B}\left(\pi_{i} \mathcal{O}_{\mathfrak{p}_{i}}\right)$ for some $i \in I$, we obtain the space $\operatorname{Hom}_{\bar{B}\left(\pi_{i} \mathcal{O}_{\mathfrak{p}_{i}}\right)}\left(\tilde{\mathrm{H}}_{0}, A\left(\kappa_{\bar{B}}\right)\right)$ that contains $S_{\kappa, I}^{D}\left(K^{p}, A\right)$. Elements in $\operatorname{Hom}_{\bar{B}\left(\pi_{i} \mathcal{O}_{\mathfrak{p}_{i}}\right)}\left(\tilde{\mathrm{H}}_{0}, A\left(\kappa_{\bar{B}}\right)\right)$ looks like automorphic forms at the place $i \in I$, and are continuous functions on the complete homology $\tilde{\mathrm{H}}_{0}$ at all other places $i^{\prime} \neq i$. For our argument, we need a generalization of the space $\operatorname{Hom}_{\bar{B}\left(\pi_{i} \mathcal{O}_{\mathfrak{p}_{i}}\right)}\left(\tilde{\mathrm{H}}_{0}, A\left(\kappa_{\bar{B}}\right)\right)$. First we introduce some notations.

Notation 3.7.5. Let $J$ be a subset of $I$. We denote by $J^{c}$ the complement of $J$ in $I$.

(1) Let $\mathcal{O}_{p, J}:=\prod_{j \in J} \mathcal{O}_{\mathfrak{p}_{j}}, \pi_{J}:=\prod_{j \in J} \pi_{j} \in \mathcal{O}_{p, J}$ and $\operatorname{Iw}_{\pi, J}:=\prod_{j \in J} \operatorname{Iw}_{\pi_{j}}$ which is regarded as a subgroup of $\operatorname{Iw}_{\pi}$ whose $j^{\prime}$-component is the identity matrix for all $j^{\prime} \notin J$. 
(2) Similar to (1), we define subgroup $B\left(\mathcal{O}_{p, J}\right)$ (resp. $T\left(\mathcal{O}_{p, J}\right), \bar{B}\left(\pi_{J} \mathcal{O}_{p, J}\right), \bar{N}\left(\pi_{J} \mathcal{O}_{p, J}\right)$, $D\left(\mathcal{O}_{p, J}\right)$ ) of $B\left(\mathcal{O}_{p}\right)$ (resp. $\left.T\left(\mathcal{O}_{p}\right), \bar{B}\left(\pi \mathcal{O}_{p}\right), \bar{N}\left(\pi \mathcal{O}_{p}\right), D\left(\mathcal{O}_{p}\right)\right)$ and the monoid $\mathbf{M}_{\pi, J} \subset$ $\mathrm{M}_{2}\left(\mathcal{O}_{p, J}\right)$.

(3) Fix $j \in I$. We set $P_{j}^{\prime}:=\left\{g \in \operatorname{Iw}_{\pi_{j}, 1} \mid \operatorname{det}(g)=1\right\}$ and denote by $P_{j}$ the subgroup of $\operatorname{Iw}_{\pi_{j}}$ generated by $\left[\begin{array}{cc}1 & 0 \\ 0 & \Delta_{j}\end{array}\right] \subset T\left(\mathcal{O}_{\mathfrak{p}_{j}}\right)$ and $P_{j}^{\prime}$. The map $D\left(\mathcal{O}_{\mathfrak{p}_{j}}\right) \times P_{j} \rightarrow \mathrm{Iw}_{\pi_{j}}$, $(d, g) \mapsto d g$ is an isomorphism of groups (here we use the assumption that $p>2$ ). $P_{j}^{\prime}$ is the pro- $p$ normal subgroup of $P_{j}$ and $P_{j} / P_{j}^{\prime} \cong \Delta_{j}$.

(4) Let $P_{J}:=\prod_{j \in J} P_{j}$ and $P_{J}^{\prime}:=\prod_{j \in J} P_{j}^{\prime}$.

(5) We use $\chi_{J}:=\left(\nu_{J}, \mu\right): \mathcal{O}_{p, J}^{\times} \times \mathbb{Z}_{p}^{\times} \rightarrow A^{\times}$to denote the restriction to the $J$-component of a continuous homomorphism $\chi=(\nu, \mu): \mathcal{O}_{p}^{\times} \times \mathbb{Z}_{p}^{\times} \rightarrow A^{\times}$.

In the rest of this section, we fix a nonempty subset $J$ of $I$ and a continuous character $\chi_{J}=\left(\nu_{J}, \mu\right): \mathcal{O}_{p, J}^{\times} \times \mathbb{Z}_{p}^{\times} \rightarrow A^{\times}$. We define a character $\kappa_{B, J}: B\left(\mathcal{O}_{p, J}\right) \times D\left(\mathcal{O}_{p, J^{c}}\right) \rightarrow A^{\times}$by

$$
\kappa_{B, J}\left(\left[\begin{array}{cc}
a_{j} & b_{j} \\
0 & d_{j}
\end{array}\right]\right):=\nu_{j}\left(a_{j} / d_{j}\right) \mu\left(\mathrm{Nm}_{F / \mathbb{Q}}\left(d_{j}\right)\right), \text { for } j \in J,
$$

and

$$
\kappa_{B, J}\left(\left[\begin{array}{cc}
a_{j} & 0 \\
0 & a_{j}
\end{array}\right]\right):=\mu\left(\mathrm{Nm}_{F / \mathbb{Q}}\left(a_{j}\right)\right), \text { for } j \notin J
$$

We remark that if $\chi_{J}$ is the restriction of a continuous character $\chi: \mathcal{O}_{p}^{\times} \times \mathbb{Z}_{p}^{\times} \rightarrow A^{\times}$, then $\kappa_{B, J}$ is the restriction of the character $\kappa_{B}: B\left(\mathcal{O}_{p}\right) \rightarrow A^{\times}$, where $\kappa: \mathcal{O}_{p}^{\times} \times \mathcal{O}_{p}^{\times} \rightarrow A^{\times}$is the character associated to $\chi$ and $\kappa_{B}$ is the character defined in $\$ 3.3$.

Definition 3.7.6. Under the above notations, we $\operatorname{set} \mathcal{C}_{\chi_{J}}\left(\operatorname{Iw}_{\pi}, A\right):=\operatorname{Ind}_{B\left(\mathcal{O}_{p, J}\right) \times D\left(\mathcal{O}_{p, J^{c}}\right)}^{\mathrm{Iw}_{\pi}}\left(\kappa_{B, J}\right)$. In particular, when $J=I$ and $\chi=\chi_{J}$ is a character of $\mathcal{O}_{p}^{\times} \times \mathbb{Z}_{p}^{\times}, \mathcal{C}_{\chi_{J}}\left(\operatorname{Iw}_{\pi}, A\right)$ is the induced representation $\operatorname{Ind}_{B\left(\mathcal{O}_{p}\right)}^{\mathrm{Iw}_{\pi}}\left(\kappa_{B}\right)$ defined in $\$ 3.3$.

Remark 3.7.7. (1) By definition, $\mathcal{C}_{\chi_{J}}\left(\mathrm{Iw}_{\pi}, A\right)$ is an $A$-submodule of the induced representation $\operatorname{Ind}_{B\left(\mathcal{O}_{p, J}\right)}^{\mathrm{Iw}_{\pi}}\left(\kappa_{J}\right) \subset \mathcal{C}\left(\operatorname{Iw}_{\pi}, A\right)$. Recall that we have defined a right action of $\mathrm{Iw}_{\pi}$ on $\mathcal{C}\left(\mathrm{Iw}_{\pi}, A\right)$ via the formula $f \circ u(g)=f\left(g u^{*}\right)$ for $f \in \mathcal{C}\left(\operatorname{Iw}_{\pi}, A\right)$ and $u \in \mathrm{Iw}_{\pi}$. This action leaves the two spaces $\mathcal{C}_{\chi_{J}}\left(\mathrm{Iw}_{\pi}, A\right)$ and $\operatorname{Ind}_{B\left(\mathcal{O}_{p, J}\right)}^{\mathrm{Iw}_{\pi}}\left(\kappa_{J}\right)$ stable.

(2) Under the Iwasawa decomposition, $\operatorname{Ind}_{B\left(\mathcal{O}_{p, J}\right)}^{\mathrm{Iw}_{\pi}}\left(\kappa_{J}\right)$ can be identified with $\mathcal{C}\left(\mathcal{O}_{p, J} \times\right.$ $\left.\operatorname{Iw}_{\pi, J^{c}}, A\right)$, and the $\mathrm{Iw}_{\pi^{-}}$-action on the latter space is given by the following explicit formula:

$$
h\left(z_{J}, g_{J^{c}}\right) \circ u=\left(\prod_{j \in J} n_{j}\left(c_{j} z_{j}+d_{j}\right) v_{j}\left(\operatorname{det}\left(u_{j}\right)\right)\right) h\left(z_{J}^{\prime}, g_{J^{c}} u_{J^{c}}^{*}\right),
$$

where $z_{J}=\left(z_{j}\right)_{j \in J} \in \mathcal{O}_{p, J}, g_{J^{c}}=\left(g_{j^{\prime}}\right)_{j^{\prime} \in J^{c}} \in \operatorname{Iw}_{\pi, J^{c}}$ and $z_{J}^{\prime}=\left(\frac{a_{j} z_{j}+b_{j}}{c_{j} z_{j}+d_{j}}\right)_{j \in J} \in \mathcal{O}_{p, J}$. Using this formula, we can extend this action to the monoid $\mathbf{M}_{\pi, J} \times \operatorname{Iw}_{\pi, J^{c}}$. This action also leaves $\mathcal{C}_{\chi_{J}}\left(\mathrm{Iw}_{\pi}, A\right)$ stable.

(3) Under the Iwasawa decomposition and the bijection $D\left(\mathcal{O}_{\mathfrak{p}_{j}}\right) \times P_{j} \rightarrow \mathrm{Iw}_{\pi_{j}}$ for all $j \in J^{c}$, we can identify $\mathcal{C}_{\chi_{J}}\left(\operatorname{Iw}_{\pi}, A\right)$ with $\mathcal{C}\left(\mathcal{O}_{p, J} \times P_{J^{c}}, A\right)$.

(4) For $\alpha \in \mathcal{O}_{p}^{\times}$, the diagonal matrix $\operatorname{Diag}(\alpha):=\left[\begin{array}{cc}\alpha & 0 \\ 0 & \alpha\end{array}\right] \in D\left(\mathcal{O}_{p}\right) \subset \operatorname{Iw}_{\pi}$ with $\alpha$ on the diagonal acts on $\mathcal{C}_{\chi_{J}}\left(\operatorname{Iw}_{\pi}, A\right)$ via multiplication by $\mu\left(\mathrm{Nm}_{F / \mathbb{Q}}(\alpha)\right)$. This fact will be used in 3.9 . 
Definition 3.7.8. Given a nonempty subset $J$ of $I$ and a continuous character $\chi_{J}: \mathcal{O}_{p, J}^{\times} \times$ $\mathbb{Z}_{p}^{\times} \rightarrow A^{\times}$, we define the space of generalized integral $p$-adic automorphic forms for $D$ to be $S_{\kappa, J}^{D}\left(K^{p}, A\right):=\left\{\phi: D^{\times} \backslash D_{f}^{\times} / K^{p} \rightarrow \mathcal{C}_{\chi_{J}}\left(\mathrm{Iw}_{\pi}, A\right) \mid \phi(x u)=\phi(x) \circ u\right.$, for all $\left.x \in D_{f}^{\times}, u \in \mathrm{Iw}_{\pi}\right\}$.

For $j \in J$, we can use the exact formula (3.4) to define the $U_{\pi_{j}}$-operator on $S_{\kappa, J}^{D}\left(K^{p}, A\right)$.

Remark 3.7.9. The reason we use the notation $S_{\kappa, J}^{D}\left(K^{p}, A\right)$ to denote the space of generalized integral $p$-adic automorphic forms is that we want to make it compatible with Definition 3.7.1, i.e. when $J=I, S_{\kappa, I}^{D}\left(K^{p}, A\right)$ defined above coincides with the space defined in Definition 3.7.1. But we point out that the definition of $S_{\kappa, J}^{D}\left(K^{p}, A\right)$ only depends on the character $\chi_{J}$ of $\mathcal{O}_{p, J}^{\times} \times \mathbb{Z}_{p}^{\times}$, even though it may be the restriction of a character $\chi$ of $\mathcal{O}_{p}^{\times} \times \mathbb{Z}_{p}^{\times}$

Remark 3.7.10. Let $J_{1} \subset J_{2}$ be two nonempty subsets of $I$ and $\chi_{J_{2}}: \mathcal{O}_{p, J_{2}}^{\times} \times \mathbb{Z}_{p}^{\times} \rightarrow A^{\times}$be a continuous character. Let $\chi_{J_{1}}$ be the restriction of $\chi_{J_{2}}$ to $\mathcal{O}_{p, J_{1}}^{\times} \times \mathbb{Z}_{p}^{\times}$. For later argument, it is useful to explain the relation between the two spaces $S_{\kappa, J_{1}}^{D}\left(K^{p}, A\right)$ and $S_{\kappa, J_{2}}^{D}\left(K^{p}, A\right)$.

(1) Since $B\left(\mathcal{O}_{p, J_{1}}\right) \times D\left(\mathcal{O}_{p, J_{1}^{c}}\right)$ is a subgroup of $B\left(\mathcal{O}_{p, J_{2}}\right) \times D\left(\mathcal{O}_{p, J_{2}^{c}}\right)$, we have a natural injection $\mathcal{C}_{\chi_{J_{2}}}\left(\mathrm{Iw}_{\pi}, A\right) \hookrightarrow \mathcal{C}_{\chi_{J_{1}}}\left(\mathrm{Iw}_{\pi}, A\right)$, which is equivariant under the action of the monoid $\mathbf{M}_{\pi, J_{1}} \times \mathrm{Iw}_{\pi, J_{1}^{c}}$. This induces an injection $S_{\kappa, J_{2}}^{D}\left(K^{p}, A\right) \rightarrow S_{\kappa, J_{1}}^{D}\left(K^{p}, A\right)$. This map is compatible with the $U_{\pi_{j}}$-operator on these spaces for all $j \in J_{1}$.

(2) We can define a right action of $B\left(\mathcal{O}_{p, J^{c}}\right)$ on $\mathcal{C}\left(\mathcal{O}_{p, J} \times \operatorname{Iw}_{\pi, J^{c}}, A\right)$ by $\left(h \cdot b_{J^{c}}\right)\left(z_{J}, g_{J^{c}}\right):=$ $h\left(z_{J}, b_{J^{c}} g_{J^{c}}\right)$ for $b_{J^{c}} \in B\left(\mathcal{O}_{p, J^{c}}\right)$ and $h \in \mathcal{C}\left(\mathcal{O}_{p, J} \times \mathrm{Iw}_{\pi, J^{c}}, A\right)$. This action leaves $\mathcal{C}_{\chi_{J}}\left(\operatorname{Iw}_{\pi}, A\right)$ stable as $D\left(\mathcal{O}_{p, J^{c}}\right)$ is central in $\operatorname{Iw}_{\pi, J^{c}}$ and hence induces a right action of $B\left(\mathcal{O}_{p, J^{c}}\right)$ on $S_{\kappa, J}^{D}\left(K^{p}, A\right)$. Set $J_{3}:=J_{2} \backslash J_{1}$. Then the injection $\mathcal{C}_{\chi_{J_{2}}}\left(\mathrm{Iw}_{\pi}, A\right) \hookrightarrow$ $\mathcal{C}_{\chi_{J_{1}}}\left(\mathrm{Iw}_{\pi}, A\right)\left(\right.$ resp. $\left.S_{\kappa, J_{2}}^{D}\left(K^{p}, A\right) \rightarrow S_{\kappa, J_{1}}^{D}\left(K^{p}, A\right)\right)$ identifies the first space with the subspace of the latter space on which the Borel subgroup $B\left(\mathcal{O}_{p, J_{3}}\right)$ acts via the character $\kappa_{B, J_{2}}$ defined above.

3.8. Spaces of classical automorphic forms. There are three goals in this section.

(1) Recall the definitions of classical weights and spaces of classical automorphic forms as defined in [5].

(2) Prove an Atkin-Lehner duality result (see Proposition 3.8.4 below).

(3) Explain how to realize some spaces of classical automorphic forms as subspaces of the space of generalized integral $p$-adic automorphic forms.

Let $\nu=\left(\nu_{i}\right)_{i \in I} \in \mathbb{Z}^{I}$ and $\mu \in \mathbb{Z}$, such that $n=\left(n_{i}:=\mu-2 \nu_{i}\right)_{i \in I} \in \mathbb{N}^{I}$. Define a character $\chi: \mathcal{O}_{p}^{\times} \times \mathbb{Z}_{p}^{\times} \rightarrow \mathbb{Q}_{p}^{\times},(\alpha, \beta) \mapsto \beta^{r} \prod_{i \in I} i_{p}\left(\alpha_{i}\right)^{\nu_{i}}$. The associated character $\kappa: \mathcal{O}_{p}^{\times} \times \mathcal{O}_{p}^{\times} \rightarrow \mathbb{Q}_{p}^{\times}$ is given by $\kappa(\alpha, \beta)=\prod_{i \in I} i_{p}\left(\alpha_{i}\right)^{n_{i}} i_{p}\left(\beta_{i}\right)^{\nu_{i}}$. We call such a weight algebraic. Let $L$ be a finite extension of $\mathbb{Q}_{p}$. A weight $\kappa: \mathcal{O}_{p}^{\times} \times \mathcal{O}_{p}^{\times} \rightarrow L^{\times}$is called locally algebraic, or classical, if $\kappa$ decomposes as $\kappa=\kappa_{\text {alg }} \kappa_{f i n}$, where $\kappa_{\text {alg }}\left(\right.$ resp. $\left.\kappa_{\text {fin }}=\psi=\left(\psi_{1}, \psi_{2}\right): \mathcal{O}_{p}^{\times} \times \mathcal{O}_{p}^{\times} \rightarrow L^{\times}\right)$ is an algebraic weight (resp. a finite character). Hence a locally algebraic weight can be represented by a triple $\left(\left(n_{i}\right)_{i \in I} \in \mathbb{N}^{I},\left(\nu_{i}\right)_{i \in I} \in \mathbb{Z}^{I}, \psi=\left(\psi_{1}, \psi_{2}\right)\right)$ with the property that $n+2 \nu \in \mathbb{Z}$ and the character $\psi_{1} \cdot \psi_{2}^{2}: \mathcal{O}_{p}^{\times} \rightarrow L^{\times}$factors through the norm map $\mathrm{Nm}_{F / \mathbb{Q}}: \mathcal{O}_{p}^{\times} \rightarrow \mathbb{Z}_{p}^{\times}$(see Convention 3.6.2.

Fix such a locally algebraic weight $\kappa=\left(n=\left(n_{i}\right)_{i \in I}, \nu=\left(\nu_{i}\right)_{i \in I}, \psi\right)$. Let $r(\kappa):=$ $\left(p^{-m_{\kappa, i}}\right)_{i \in I}$ and $t:=\left(t_{i}\right)_{i \in I} \in \mathbb{N}^{I}$ such that $\psi: \mathcal{O}_{p}^{\times} \times \mathcal{O}_{p}^{\times} \rightarrow L^{\times}$factors through $\left(\mathcal{O}_{p} / \pi^{t}\right)^{\times} \times$ $\left(\mathcal{O}_{p} / \pi^{t}\right)^{\times}$. It is straightforward to verify that $t_{i} \geqslant m_{\kappa, i}$ for all $i \in I$. In particular, $t$ is good for $(\kappa, r)$ for all $r \in \mathcal{N}^{I}$. From the integer $\nu_{i}$ and the character $\psi_{2, i}: \mathcal{O}_{\mathfrak{p}_{i}}^{\times} \rightarrow L^{\times}$, we define 
another character $\tau_{i}: \mathcal{O}_{\mathfrak{p}_{i}}^{\times} \rightarrow L^{\times}, \beta_{i} \mapsto i_{p}\left(\beta_{i}\right)^{\nu_{i}} \psi_{2, i}\left(\beta_{i}\right)$ and extend it to a character of $F_{\mathfrak{p}_{i}}^{\times}$ by setting $\tau_{i}\left(\pi_{i}\right)=1$.

We put $L_{\kappa}$ to be the $L$-vector space with basis $\Sigma_{\kappa}:=\left\{\prod_{i \in I} Z_{i}^{l_{i}} \mid 0 \leqslant l_{i} \leqslant n_{i}\right.$, for all $\left.i\right\}$, where $Z_{i}$ 's are independent indeterminates. The space $L_{\kappa}$ carries a right action of $\mathbf{M}_{\pi^{t}}$ by

$$
\left(\prod_{i \in I} Z_{i}^{l_{i}}\right) \circ \gamma=\prod_{i \in I} \psi_{1, i}\left(d_{i}\right) \tau_{i}\left(\operatorname{det}\left(\gamma_{i}\right)\right)\left(a_{i} Z_{i}+b_{i}\right)^{l_{i}}\left(c_{i} Z_{i}+d_{i}\right)^{n_{i}-l_{i}},
$$

for $\gamma=\left(\gamma_{i}\right)_{i \in I} \in M_{\pi^{t}}$ with $\gamma_{i}=\left[\begin{array}{cc}a_{i} & b_{i} \\ c_{i} & d_{i}\end{array}\right]$ for $i \in I$.

Let $\mathcal{O}_{L}$ be the ring of integers of $L$. We denote by $L_{\kappa, \mathcal{O}_{L}}$ the $\mathcal{O}_{L}$-lattice of $L_{\kappa}$ spanned by the polynomials in $\Sigma_{\kappa}$. Since the character $\kappa$ takes values in $\mathcal{O}_{L}^{\times}$, the action of the monoid $\mathbf{M}_{\pi^{t}}$ on $L_{\kappa}$ leaves $L_{\kappa, \mathcal{O}_{L}}$ stable.

Fix an open compact subgroup $K^{p}$ of $D_{f}^{(p), \times}$ and let $K=K^{p} \operatorname{Iw}_{\pi^{t}}$ be the subgroup of $D_{f}^{\times}$.

Definition 3.8.1. Under the above notations, define $k=n+2 \in \mathbb{Z}_{\geqslant 2}^{I}$ and $w=n+\nu+1 \in \mathbb{Z}^{I}$. The space of classical automorphic forms of weight $(k, w)$, level $K$ and character $\psi$ for $D$ is defined by

$$
S_{k, w}^{D}(K, \psi):=\left\{\phi: D^{\times} \backslash D_{f}^{\times} / K^{p} \rightarrow L_{\kappa} \mid \phi(x u)=\phi(x) \circ u, \text { for all } x \in D_{f}^{\times}, u \in \operatorname{Iw}_{\pi^{t}}\right\} .
$$

We also define

$$
S_{k, w}^{D}\left(K, \psi, \mathcal{O}_{L}\right):=\left\{\phi: D^{\times} \backslash D_{f}^{\times} / K^{p} \rightarrow L_{\kappa, \mathcal{O}_{L}} \mid \phi(x u)=\phi(x) \circ u \text {, for all } x \in D_{f}^{\times}, u \in \mathrm{Iw}_{\pi^{t}}\right\} .
$$

Note that $S_{k, w}^{D}\left(K, \psi, \mathcal{O}_{L}\right)$ is an $\mathcal{O}_{L}$-lattice of $S_{k, w}^{D}(K, \psi)$ and stable under the $U_{\pi_{i}}$-operators for all $i \in I$.

Remark 3.8.2. Convention 3.3 .1 on the weight $\kappa=(n, \nu): \mathcal{O}_{p}^{\times} \times \mathcal{O}_{p}^{\times} \rightarrow L^{\times}$makes our definition of Hecke operators on the spaces of classical automorphic forms slightly different from the 'usual' one (see [15, §2] for example). To be more precise, we assume that the character $\psi$ above is trivial for simplicity. We will define a second action of the monoid $\mathbf{M}_{\pi^{t}}$ on $L_{\kappa}$ below. This action coincides with the one that we defined above when restricting to $\mathrm{Iw}_{\pi^{t}}$. In particular, it defines the same spaces of classical automorphic forms. However, this two actions differ by a power of $\pi$ as an action of the monoid $\mathbf{M}_{\pi^{t}}$, and hence define different Hecke operators. If we use $U_{\pi_{i}, c l}$ to denote the Hecke operator defined by the second action $\|_{\gamma}$, then it is related with our Hecke operator $U_{\pi_{i}}$ defined in $\$ 3.5$ via the equality $U_{\pi_{i}, c l}=\pi_{i}^{\nu_{i}} U_{\pi_{i}}$. The reason that we renormalize the Hecke operators is that the quantity $\pi_{i}^{\nu_{i}}$ does not vary analytically with $\nu$.

We keep the notations as before. We define another right action of the monoid $\mathbf{M}_{\pi^{t}}$ on $L_{\kappa}$ by

$$
\left(\prod_{i \in I} Z_{i}^{l_{i}}\right) \|_{\gamma}:=\prod_{i \in I}\left(\operatorname{det}\left(\gamma_{i}\right)\right)^{\nu_{i}}\left(\left(a_{i} Z_{i}+b_{i}\right)^{l_{i}}\left(c_{i} Z_{i}+d_{i}\right)^{n_{i}-l_{i}}\right)
$$

for $\gamma=\left(\gamma_{i}\right)_{i \in I} \in \mathbf{M}_{\pi^{t}}$ and $\gamma_{i}=\left[\begin{array}{cc}a_{i} & b_{i} \\ c_{i} & d_{i}\end{array}\right]$ for all $i \in I$. Then the space of classical automorphic forms can be described by

$$
\begin{array}{r}
S_{k, w}^{D}(K, \psi)=\left\{\phi: D^{\times} \backslash D_{f}^{\times} / K^{p} \rightarrow L_{\kappa} \mid \phi(x u)=\psi_{1}(d) \psi_{2}(\operatorname{det}(u)) \phi(x) \|_{u}\right. \\
\text { for } \left.x \in D_{f}^{\times}, u=\left[\begin{array}{ll}
a & b \\
c & d
\end{array}\right] \in \operatorname{Iw}_{\pi^{t}}\right\} .
\end{array}
$$


Note that since $\psi_{2}$ factors through $\left(\mathcal{O}_{p} / \pi^{t}\right)^{\times}$, we have $\psi_{2}(\operatorname{det}(u))=\psi_{2}(a d)$.

Fix $j \in I$. Define another finite character $\psi^{\prime}=\left(\psi_{1}^{\prime}, \psi_{2}^{\prime}\right): \mathcal{O}_{p}^{\times} \times \mathcal{O}_{p}^{\times} \rightarrow L^{\times}$by setting:

$$
\psi_{1, i}^{\prime}:=\left\{\begin{array}{ll}
\psi_{1, i}, & \text { if } i \neq j \\
\psi_{1, i}^{-1}, & \text { if } i=j
\end{array} \text { and } \psi_{2, i}^{\prime}:=\left\{\begin{array}{ll}
\psi_{2, i}, & \text { if } i \neq j \\
\psi_{1, i} \psi_{2, i}, & \text { if } i=j
\end{array} .\right.\right.
$$

In other words, if we regard $\psi_{1, j}$ as a character of $\mathcal{O}_{p}^{\times}$via the natural projection $\mathcal{O}_{p}^{\times} \rightarrow \mathcal{O}_{\mathfrak{p}_{j}}^{\times}$, we have $\psi_{1}^{\prime}=\psi_{1} \psi_{1, j}^{-2}$ and $\psi_{2}^{\prime}=\psi_{2} \psi_{1, j}$.

The Atkin-Lehner map is defined by

$$
\mathrm{AL}_{j}: S_{k, w}^{D}(K, \psi) \rightarrow S_{k, w}^{D}\left(K, \psi^{\prime}\right), \phi \mapsto \phi\left(\bullet v_{j}^{-1}\right) \|_{v_{j}},
$$

where $v_{j}=\left[\begin{array}{cc}0 & 1 \\ \pi_{j}^{t_{j}} & 0\end{array}\right] \in \mathbf{M}_{\pi_{j}^{t_{j}}}$ and we view $v_{j}$ as an element of $\mathbf{M}_{\pi^{t}}$ whose $i$-component is the identity, for all $i \neq j$. First we verify that the map $\mathrm{AL}_{j}$ is well-defined. Given $\phi \in$ $S_{k, w}^{D}(U, \psi), x \in D_{f}^{\times}$and $u_{j}=\left[\begin{array}{cc}a_{j} & b_{j} \\ c_{j} & d_{j}\end{array}\right] \in \mathrm{Iw}_{\pi_{j}^{t_{j}}}$, we set $\tilde{u}_{j}:=v_{j} u_{j} v_{j}^{-1}=\left[\begin{array}{cc}d_{j} & \pi_{j}^{-t_{j}} c_{j} \\ \pi_{j}^{t_{j}} b_{j} & a_{j}\end{array}\right]$. By a direct computation, we have

$$
\begin{aligned}
\mathrm{AL}_{j}(\phi)\left(x u_{j}\right) & =\phi\left(x u_{j} v_{j}^{-1}\right)\left\|_{v_{j}}=\phi\left(x v_{j}^{-1} \tilde{u}_{j}\right)\right\|_{v_{j}}=\left(\psi_{1, j}\left(a_{j}\right) \psi_{2, j}\left(\operatorname{det}\left(\tilde{u}_{j}\right)\right) \phi\left(x v_{j}^{-1}\right) \|_{\tilde{u}_{j}}\right) \|_{v_{j}} \\
& =\psi_{1, j}\left(a_{j}\right) \psi_{2, j}\left(\operatorname{det}\left(\tilde{u}_{j}\right)\right) \phi\left(x v_{j}^{-1}\right)\left\|_{\tilde{u}_{j} v_{j}}=\psi_{1, j}\left(a_{j}\right) \psi_{2, j}\left(\operatorname{det}\left(\tilde{u}_{j}\right)\right)\left(\phi\left(x v_{j}^{-1}\right) \|_{v_{j}}\right)\right\|_{u_{j}} \\
& =\psi_{1, j}^{-1}\left(d_{j}\right) \psi_{1, j}\left(a_{j} d_{j}\right) \psi_{2, j}\left(\operatorname{det}\left(u_{j}\right)\right) \operatorname{AL}_{j}(\phi)(x)\left\|_{u_{j}}=\psi_{1, j}^{\prime}\left(d_{j}\right) \psi_{2, j}^{\prime}\left(\operatorname{det}\left(u_{j}\right)\right) \operatorname{AL}_{j}(\phi)(x)\right\|_{u_{j}} .
\end{aligned}
$$

Thus, we know that $\mathrm{AL}_{j}(\phi) \in S_{k, w}^{D}\left(K, \psi^{\prime}\right)$ and $\mathrm{AL}_{j}$ is well-defined. It is clear that $\mathrm{AL}_{j}$ is an $L$-linear isomorphism and induces an $\mathcal{O}_{L}$-linear isomorphism between $S_{k, w}^{D}\left(K, \psi, \mathcal{O}_{L}\right)$ and $S_{k, w}^{D}\left(K, \psi^{\prime}, \mathcal{O}_{L}\right)$.

Definition 3.8.3. The locally algebraic weight $\kappa^{\prime}$ corresponding to the triple $\left(n, v, \psi^{\prime}\right)$ is called the $j$-Atkin-Lehner dual weight of $\kappa$.

Proposition 3.8.4. Assume that the finite character $\psi_{1, j}: \mathcal{O}_{\mathfrak{p}_{j}}^{\times} \rightarrow L^{\times}$has conductor $t_{j} \geqslant 2$. For $\phi \in S_{k, w}^{D}(K, \psi)$, we have $U_{\pi_{j}} \circ \mathrm{AL}_{j} \circ U_{\pi_{j}}(\phi)=p \pi_{j}^{n_{j}} \mathrm{AL}_{j} \circ S_{\pi_{j}}(\phi)$, where $S_{\pi_{j}}: S_{k, w}^{D}(K, \psi) \rightarrow$ $S_{k, w}^{D}(K, \psi)$ is the automorphism defined by $\phi \mapsto \phi\left(\bullet\left[\begin{array}{cc}\pi_{j}^{-1} & 0 \\ 0 & \pi_{j}^{-1}\end{array}\right]\right)$.

Proof. First we make a remark on notations. In this proposition, we have two locally algebraic weights $\kappa=(n, v, \psi)$ and $\kappa^{\prime}=\left(n, v, \psi^{\prime}\right)$, and hence two different actions of the monoid $\mathbf{M}_{\pi^{t}}$ on the $L$-vector space $L_{\kappa}=L_{\kappa^{\prime}}$. However, for $v_{j, l}=\left[\begin{array}{cc}\pi_{j} & 0 \\ l \pi_{j}^{t_{j}} & 1\end{array}\right], l=0, \ldots, p-$ 1 , the right action of $v_{j, l}$ on $L_{\kappa}=L_{\kappa^{\prime}}$ is given by $h \circ v_{j, l}=h \|_{v_{j, l}}$, no matter which weights $\kappa$ or $\kappa^{\prime}$ that we consider. Hence for $\phi \in S_{k, w}^{D}(K, \psi)$ or $S_{k, w}^{D}\left(K, \psi^{\prime}\right)$, we always have $U_{\pi_{j}}(\phi)(x)=$ $\sum_{l=0}^{p-1} \phi\left(x v_{j, l}^{-1}\right) \|_{v_{j, l}}$.

For $x \in D_{f}^{\times}$and $\phi \in S_{k, w}^{D}(K, \psi)$, we have

$U_{\pi_{j}} \circ \mathrm{AL}_{j} \circ U_{\pi_{j}}(\phi)(x)=\sum_{l, m=0}^{p-1} \phi\left(x v_{j, m}^{-1} v_{j}^{-1} v_{j, l}^{-1}\right)\left\|_{v_{j, l} v_{j} v_{j, m}}=\sum_{l, m=0}^{p-1} \phi\left(x v_{j}^{-1}\left(v_{j} v_{j, m}^{-1} v_{j}^{-1} v_{j, l}^{-1}\right)\right)\right\|_{v_{j, l} v_{j} v_{j, m}}$.

By a direct computation, we have

$v_{j} v_{j, m}^{-1} v_{j}^{-1} v_{j, l}^{-1}=\left[\begin{array}{cc}1 & -m \pi_{j}^{-1} \\ 0 & \pi_{j}^{-1}\end{array}\right]\left[\begin{array}{cc}\pi_{j}^{-1} & 0 \\ -l \pi_{j}^{t_{j}-1} & 1\end{array}\right]=\left[\begin{array}{cc}\pi_{j}^{-1} & 0 \\ -l \pi_{j}^{t_{j}-2} & \pi_{j}^{-1}\end{array}\right]\left[\begin{array}{cc}1+l m \pi_{j}^{t_{j}-1} & -m \\ m l^{2} \pi_{j}^{2 t_{j}-2} & 1-l m \pi_{j}^{t_{j}-1}\end{array}\right]$. 
From the hypothesis $t_{j} \geqslant 2$, the matrix $\left[\begin{array}{cc}1+l m \pi_{j}^{t_{j}-1} & -m \\ m l^{2} \pi_{j}^{2 t_{j}-2} & 1-l m \pi_{j}^{t_{j}-1}\end{array}\right]$ belongs to $\mathrm{Iw}_{\pi_{j}} t_{j}$. Hence, we have

$\phi\left(x v_{j}^{-1}\left(v_{j} v_{j, m}^{-1} v_{j}^{-1} v_{j, l}^{-1}\right)\right)=\psi_{1, j}\left(1-l m \pi_{j}^{t_{j}-1}\right) \phi\left(x v_{j}^{-1}\left[\begin{array}{cc}\pi_{j}^{-1} & 0 \\ -l \pi_{j}^{t_{j}-2} & \pi_{j}^{-1}\end{array}\right]\right) \|\left[\begin{array}{cc}1+l m \pi_{j}^{t_{j}-1} & -m \\ m l^{2} \pi_{j}^{2 t_{j}-2} & 1-l m \pi_{j}^{t_{j}-1}\end{array}\right]$,

and consequently

$$
\begin{aligned}
U_{\pi_{j}} \circ \mathrm{AL}_{j} \circ U_{\pi_{j}}(\phi)(x) \\
\quad=\sum_{l, m=0}^{p-1} \psi_{1, j}\left(1-l m \pi_{j}^{t_{j}-1}\right) \phi\left(x v_{j}^{-1}\left[\begin{array}{cc}
\pi_{j}^{-1} & 0 \\
-l \pi_{j}^{t_{j}-2} & \pi_{j}^{-1}
\end{array}\right]\right) \|\left[\begin{array}{cc}
1+l m \pi_{j}^{t_{j}-1} & -m \\
m l^{2} \pi_{j}^{2 t_{j}-2} & 1-l m \pi_{j}^{t_{j} j-1}
\end{array}\right] v_{j, l} v_{j} v_{j, m}
\end{aligned} .
$$

Since $\psi_{1, j}$ is a finite character of conductor $\pi_{j}^{t_{j}}$, we have

$$
\sum_{m=0}^{p-1} \psi_{1, j}\left(1-l m \pi_{j}^{t_{j}-1}\right)=\left\{\begin{array}{lc}
p, & \text { for } l=0 \\
0, & \text { otherwise }
\end{array}\right.
$$

and hence

$$
U_{\pi_{j}} \circ \mathrm{AL}_{j} \circ U_{\pi_{j}}(\phi)(x)=p \phi\left(x v_{j}^{-1}\left[\begin{array}{cc}
\pi_{j}^{-1} & 0 \\
0 & \pi_{j}^{-1}
\end{array}\right]\right) \|\left[\begin{array}{cc}
\pi_{j} & 0 \\
0 & \pi_{j}
\end{array}\right] v_{j}=p \pi_{j}^{n_{j}} \mathrm{AL}_{j} \circ S_{\pi_{j}}(\phi)(x) .
$$

Proposition 3.8.5. The slopes of the $U_{\pi_{j}}$ operator on the two spaces $S_{k, w}^{D}(K, \psi)$ and $S_{k, w}^{D}\left(K, \psi^{\prime}\right)$ can be paired such that the slopes in each pair sum to $n_{i}+1=k_{i}-1$.

We will give the proof of this proposition after introducing the following lemma.

Lemma 3.8.6. Let $L$ be a field with a valuation $v_{L}(\cdot)$ and $\mathcal{O}_{L}$ be its valuation ring. Consider three matrices $A, B \in \mathrm{M}_{n}(L)$ and $U \in \mathrm{GL}_{n}\left(\mathcal{O}_{L}\right)$, such that $U$ commutes with $B$ and $A B=$ $\alpha U$ for some $\alpha \in \mathcal{O}_{L}$ with $v_{L}(\alpha)=k$. Then one can pair the slopes of the Newton polygons of $A$ and $B$, such that the slopes in each pair sum to $k$.

Proof. Replacing $L$ by its algebraic closure $\bar{L}$ and extending $v_{L}(\cdot)$ to $\bar{L}$, we can assume that $L$ is algebraically closed. We regard $A, B, U$ as $L$-linear operators on the space $V=L^{n}$. Then $V$ has the decomposition $V=\oplus \quad V_{\lambda}$, where $V_{\lambda}=\operatorname{ker}\left(\left(U-\lambda I_{n}\right)^{n}\right)$. Since $A B=\alpha U$ and $U$ commutes with $B$, the three matrices $A, B, U$ all commute with the others. Hence $A, B$ stabilizes the spaces $V_{\lambda}$ 's. Hence we may assume that $V=\operatorname{ker}\left(\left(U-\lambda I_{n}\right)^{n}\right)$ for some $\lambda \in L$. For the same reason, we can also assume that $V=\operatorname{ker}\left(\left(A-\lambda_{A} I_{n}\right)^{n}\right)$ for some $\lambda_{A} \in L$. Since $U \in \operatorname{GL}_{n}\left(\mathcal{O}_{L}\right)$, we have $\lambda \in \mathcal{O}_{L}^{\times}$and hence $v_{L}(\lambda)=0$. Let $\lambda_{B}=a \lambda \lambda_{A}^{-1}$. Then $B-\lambda_{B} I_{n}=\alpha A^{-1} U-\alpha \lambda \lambda_{A}^{-1}=\alpha \lambda_{A}^{-1} A^{-1}\left(\lambda_{A} U-\lambda A\right)=\alpha \lambda_{A}^{-1} A^{-1}\left(\lambda_{A}\left(U-\lambda I_{n}\right)+\lambda\left(\lambda_{A} I_{n}-\right.\right.$ $A)$ ). Therefore $B-\lambda_{B} I_{n}$ is a nilpotent operator on $V$. The eigenvalues of $B$ are $\lambda_{B}$ with multiplicity $n$. Since $\lambda_{A} \lambda_{B}=\alpha \lambda$, we have $v_{L}\left(\lambda_{A}\right)+v_{L}\left(\lambda_{B}\right)=v_{L}(\alpha)+v_{L}(\lambda)=k$.

Proof of Proposition 3.8.5. We choose an $\mathcal{O}_{L}$-basis $\Omega$ of $S_{k, w}^{D}\left(K, \psi, \mathcal{O}_{L}\right)$ which can be viewed as an $L$-basis of $S_{k, w}^{D}(K, \psi)$. We denote by $A \in \mathrm{M}_{n}(L)$ (resp. $B \in \mathrm{M}_{n}(L)$, resp. $U \in$ $\left.\mathrm{GL}_{n}\left(\mathcal{O}_{L}\right)\right)$ the matrix corresponding to the map $\mathrm{AL}_{j}^{-1} \circ U_{\pi_{j}} \circ \mathrm{AL}_{j}: S_{k, w}^{D}(K, \psi) \rightarrow S_{k, w}^{D}(K, \psi)$ (resp. $U_{\pi_{j}}: S_{k, w}^{D}(K, \psi) \rightarrow S_{k, w}^{D}(K, \psi)$, resp. $\left.S_{\pi_{j}}: S_{k, w}^{D}(K, \psi) \rightarrow S_{k, w}^{D}(K, \psi)\right)$. 
Notice that the operator $S_{\pi_{j}}$ preserves $S_{k, w}^{D}\left(K, \psi, \mathcal{O}_{L}\right)$ and commutes with $U_{\pi_{j}}$ as $\left[\begin{array}{cc}\pi_{j}^{-1} & 0 \\ 0 & \pi_{j}^{-1}\end{array}\right]$ is central in $\mathrm{GL}_{2}\left(F_{\mathfrak{p}_{j}}\right)$. Now the proposition follows from Proposition 3.8.4
and Lemma 3.8.6.

Fix a nonempty subset $J$ of $I$ and a locally algebraic weight $\kappa$ corresponding to the triple $\left(\left(n_{i}\right)_{i \in I} \in \mathbb{Z}_{\geqslant 0}^{I},\left(\nu_{i}\right)_{i \in I} \in \mathbb{Z}^{I}, \psi=\left(\psi_{1}, \psi_{2}\right)\right)$ with the following property: $n_{j}=\nu_{j}=0$ and

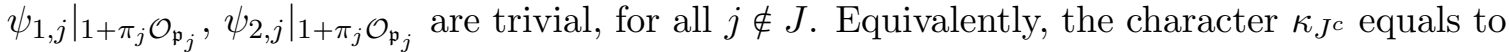
$\psi_{J^{c}}=\left(\psi_{1, J^{c}}, \psi_{2, J^{c}}\right): \mathcal{O}_{p, J^{c}}^{\times} \times \mathcal{O}_{p, J^{c}}^{\times} \rightarrow L^{\times}$and factors through $\left(\mathcal{O}_{p, J^{c}} / \pi_{J^{c}}\right)^{\times} \times\left(\mathcal{O}_{p, J^{c}} / \pi_{J^{c}}\right)^{\times}$. The character $\kappa_{J^{c}, T}: T\left(\mathcal{O}_{p, J^{c}}\right) \rightarrow L^{\times}$is trivial on $T\left(1+\pi_{J^{c}} \mathcal{O}_{p, J^{c}}\right)$ under the decomposition $T\left(\mathcal{O}_{p, J^{c}}\right)=T\left(\mathcal{O}_{p, J^{c}} / \pi_{J^{c}}\right) \times T\left(1+\pi_{J^{c}} \mathcal{O}_{p, J^{c}}\right)$.

Since $\mathrm{Iw}_{\pi, 1, J^{c}}$ is a normal subgroup of $\mathrm{Iw}_{\pi, J^{c}}$ with quotient $\operatorname{Iw}_{\pi, J^{c}} / \operatorname{Iw}_{\pi, 1, J^{c}} \cong T\left(\mathcal{O}_{p, J^{c}} / \pi_{J^{c}}\right)$, the character $\kappa_{T, J^{c}}: T\left(\mathcal{O}_{p, J^{c}}\right) \rightarrow L^{\times}$induces a character $\alpha_{J^{c}}: \operatorname{Iw}_{\pi, J^{c}} \rightarrow L^{\times}$. As $p$ splits in $F, \alpha_{J^{c}}$ takes values in $\Delta \subset \mathbb{Z}_{p}^{\times}$. Moreover, if the locally algebraic weight $\kappa$ is the character associated to a point $\chi \in \mathcal{W}(L)$, then the character $\alpha_{J^{c}}$ only depends on $\omega:=\left.\chi\right|_{H}$ (or equivalently, the component that $\chi$ belongs to), where $H$ is the torsion subgroup of $\mathcal{O}_{p}^{\times} \times \mathbb{Z}_{p}^{\times}$and the set $J$. We choose $t=\left(t_{i}\right)_{i \in I} \in \mathbb{N}^{I}$ such that the character $\psi$ factors through $\left(\mathcal{O}_{p} / \pi^{t}\right)^{\times} \times\left(\mathcal{O}_{p} / \pi^{t}\right)^{\times}$. In particular, we take $t_{j}=1$ for all $j \notin J$.

Definition 3.8.7. We call $\alpha_{J^{c}}$ the character associated to the character $\omega \in H^{\vee}$ and the set $J$.

For the locally algebraic weight $\kappa$ we consider above, let $k, w \in \mathbb{Z}^{I}$ be defined as in Definition 3.8.1. We can regard the $L$-vector space $L_{\kappa}$ as polynomial functions on $\mathcal{O}_{p}=\prod_{i \in I} \mathcal{O}_{\mathfrak{p}_{i}}$ and hence obtain an embedding $L_{n, v} \rightarrow \mathcal{C}\left(\mathcal{O}_{p}, L\right)$, which is equivariant under the action of the monoid $\mathbf{M}_{\pi^{t}}$. So we have an embedding $S_{k, w}^{D}(K, \psi) \rightarrow S_{\kappa, I}^{D}\left(K^{p} \operatorname{Iw}_{\pi^{t}}, L\right)$. Composing with the isomorphism $S_{\kappa, I}^{D}\left(K^{p} \operatorname{Iw}_{\pi^{t}}, L\right) \cong S_{\kappa, I}^{D}\left(K^{p}, L\right)$ established in Remark 3.7.2 and the embedding $S_{\kappa, I}^{D}\left(K^{p}, L\right) \hookrightarrow S_{\kappa, J}^{D}\left(K^{p}, L\right)$, we obtain an embedding $S_{k, w}^{D}(K, \psi) \rightarrow S_{\kappa, J}^{D}\left(K^{p}, L\right)$. Then we have the following characterization of the image of this embedding.

Proposition 3.8.8. Under the above assumptions on the weight $\kappa$, the image of the embedding $S_{k, w}^{D}(K, \psi) \rightarrow S_{\kappa, J}^{D}\left(K^{p}, L\right)$ lies in the subspace of $S_{\kappa, J}^{D}\left(K^{p}, L\right)$ on which the group $\mathrm{Iw}_{\pi, J^{c}}$ acts via the character $\alpha_{J^{c}}: \mathrm{Iw}_{\pi, J^{c}} \rightarrow \mathbb{Z}_{p}^{\times}$associated to $\omega \in H^{\vee}$ and the set $J$.

Fix a locally algebraic weight $\kappa: \mathcal{O}_{p}^{\times} \times \mathcal{O}_{p}^{\times} \rightarrow L^{\times}$that corresponds to the triple $(n, \nu, \psi)$ and we choose $t=\left(t_{i}\right)_{i \in I} \in \mathbb{N}^{I}$ such that $\psi$ factors through $\left(\mathcal{O}_{p} / \pi^{t}\right)^{\times} \times\left(\mathcal{O}_{p} / \pi^{t}\right)^{\times}$as before. Let $K=K^{p} \mathrm{Iw}_{\pi^{t}}$ be an open compact subgroup of $D_{f}^{\times}$. Fix $i \in I$. Define $n^{\prime}, \nu^{\prime} \in \mathbb{Z}^{I}$ as follows:

$$
n_{j}^{\prime}=\left\{\begin{array}{ll}
n_{j}, & \text { if } j \neq i \\
n_{j}, & \text { if } j=i
\end{array} \text { and } \nu_{j}^{\prime}=\left\{\begin{array}{ll}
\nu_{j}, & \text { if } j \neq i \\
\nu_{j}+n_{j}-1, & \text { if } j=i
\end{array} .\right.\right.
$$

Let $\kappa^{\prime}$ be the locally algebraic weight that corresponds to the triple $\left(n^{\prime}, \nu^{\prime}, \psi\right)$. Fix $r \in \mathcal{N}^{I}$ and we denote by $z_{j}$ the coordinate of the $j$-component of the polydisc $\mathbf{B}_{r}$ for all $j \in I$. Under the above notations, the differential operator $\left(\frac{d}{d z_{i}}\right)^{n_{i}+1}: \mathcal{A}_{\kappa, r} \rightarrow \mathcal{A}_{\kappa^{\prime}, r}$ induces an operator $\theta_{i}: S_{\kappa}^{D}(K, r) \rightarrow S_{\kappa^{\prime}}^{D}(K, r)$. Moreover, this map is equivariant for the $U_{\pi_{i} \text {-operator }}$ on the source and the $\pi_{i}^{n_{i}+1} U_{\pi_{i}}$-operator on the target. When $F=\mathbb{Q}$, this is proven in $[6, \S 7]$. The proof of the general case is similar. At the end of this section, we record the following classicality result that characterizes the image of $S_{k, w}^{D}(K, \psi)$ in $S_{\kappa}^{D}(K, r)$.

Proposition 3.8.9. Fix $\phi \in S_{\kappa}^{D}(K, r)$. Then 
(1) $\phi \in S_{k, w}^{D}(K, \psi)$ if and only if $\theta_{i}(\phi)=0$ for all $i \in I$.

(2) If $\phi$ is an eigenform for the $U_{\pi_{i}}$-operator with nonzero eigenvalue $\lambda_{i}$ such that $v_{p}\left(\lambda_{i}\right)<n_{i}+1$ for all $i \in I$, then $\phi \in S_{k, w}^{D}(K, \psi)$.

Proof. Part (2) follows from [2, Theorem 4.3.6]. Part (1) follows from the proof given there.

3.9. Explicit expression of $p$-adic automorphic forms. Let $A$ be a topological ring and $\kappa: \mathcal{O}_{p}^{\times} \times \mathcal{O}_{p}^{\times} \rightarrow A^{\times}$as before. Fix a double coset decomposition $D_{f}^{\times}=\bigsqcup_{k=0}^{s-1} D^{\times} \gamma_{k} K^{p} \mathrm{Iw}_{\pi}$ with $\gamma_{k} \in D_{f}^{\times}$. We have an isomorphism of $A$-modules:

$$
S_{\kappa, I}^{D}\left(K^{p}\right) \stackrel{\cong}{\rightarrow} \bigoplus_{k=0}^{s-1} \mathcal{C}\left(\mathcal{O}_{p}, A\right)^{\Gamma_{k}}, \phi \mapsto\left(\phi\left(\gamma_{k}\right)\right)_{k=0, \ldots, s-1},
$$

where $\Gamma_{k}=\gamma_{k}^{-1} D^{\times} \gamma_{k} \cap K^{p} \mathrm{Iw}_{\pi}$ and $\Gamma_{k}$ acts on $\mathcal{C}\left(\mathcal{O}_{p}, A\right)$ via its $\mathrm{GL}_{2}\left(F_{p}\right)$-component.

We embed $\mathcal{O}_{F}^{\times}$diagonally to $D_{f}^{\times}$and hence view $\mathcal{O}_{F}^{\times}$as a subgroup of $D_{f}^{\times}$. From 15 , Lemma 7.1] and the assumption that $D / F$ is totally definite, we can choose $K^{p}$ small enough such that $\Gamma_{k} \subset \mathcal{O}_{F}^{\times,+}$, for all $k=0, \ldots, s-1$. The subgroup $K^{p}$ with this property is called neat. For a neat $K^{p}$, we have the isomorphism $S_{\kappa, I}^{D}\left(K^{p}\right) \cong \bigoplus_{k=0}^{s-1} \mathcal{C}\left(\mathcal{O}_{p}, A\right)$. Similarly we have explicit descriptions of the spaces of $p$-adic overconvergent automorphic forms by evaluating the functions at $\gamma_{k}$ 's, for $k=0, \ldots, s-1: S_{\kappa}^{D}\left(K^{p} \mathrm{Iw}_{\pi}, r\right) \stackrel{\cong}{\rightarrow} \bigoplus_{k=0}^{s-1} \mathcal{A}_{\kappa, r}$, where $A$ a $\mathbb{Q}_{p}$-affinoid algebra and $(1, \ldots, 1)$ good for $(\kappa, r)$.

Convention 3.9.1. In the rest of this paper, we always assume that $K^{p}$ is neat.

In general, we fix a nonempty subset $J$ of $I$ and a continuous character $\chi_{J}=\left(\nu_{J}, \mu\right)$ : $\mathcal{O}_{p, J}^{\times} \times \mathbb{Z}_{p}^{\times} \rightarrow A^{\times}$. As observed in Remark 3.7.7, for any $\alpha \in \mathcal{O}_{F}^{\times}$, the matrix $\operatorname{Diag}(\alpha) \in T\left(\mathcal{O}_{p}\right)$ acts on $\mathcal{C}_{\chi_{J}}\left(\operatorname{Iw}_{\pi}, A\right)$ via multiplication by $\mu\left(\mathrm{Nm}_{F / \mathbb{Q}}(\alpha)\right)$. So we have an isomorphism of $A$ modules: $S_{\kappa, J}^{D}\left(K^{p}, A\right) \stackrel{s-1}{\rightrightarrows} \bigoplus_{k=0}^{s} \mathcal{C}_{\chi_{J}}\left(\operatorname{Iw}_{\pi}, A\right)$.

\section{A filtration on the space of integral $p$-ADiC automorphic Forms}

\subsection{Notations.}

- We label the elements in $I=\operatorname{Hom}(F, \overline{\mathbb{Q}})$ by $I=\left\{i_{1}, \ldots, i_{g}\right\}$. For $1 \leqslant l \leqslant g-1$, let $J_{l}=\left\{i_{1}, \ldots, i_{l}\right\} \subset I$.

- Let $H$ be the torsion subgroup of $\mathcal{O}_{p}^{\times} \times \mathbb{Z}_{p}^{\times}$. Hence $\mathcal{O}_{p}^{\times} \times \mathbb{Z}_{p}^{\times} \cong H \times\left(\left(1+\pi \mathcal{O}_{p}\right) \times\right.$ $\left.\left(1+p \mathbb{Z}_{p}\right)\right)$ and $\mathcal{W} \cong \prod_{\omega \in H^{\vee}} \mathcal{W}_{\omega}$, where $H^{\vee}$ is the character group of $H$, and $\mathcal{W}_{\omega}$ is isomorphic to the $(g+1)$-dimensional open unit polydisc.

- The homomorphism $\phi_{\rho}: \mathcal{O}_{p}^{\times} \rightarrow \mathcal{O}_{p}^{\times} \times \mathbb{Z}_{p}^{\times}$induces a continuous homomorphism $\mathbb{Z}_{p} \llbracket \mathcal{O}_{p}^{\times} \rrbracket \rightarrow \mathbb{Z}_{p} \llbracket \mathcal{O}_{p}^{\times} \times \mathbb{Z}_{p}^{\times} \rrbracket$, which is still denoted by $\phi_{\rho}$. For each $i \in I$, define $T_{i}:=\phi_{\rho}\left(\left[\exp \left(\pi_{i}\right)\right]-1\right) \in \mathbb{Z}_{p} \llbracket \mathcal{O}_{p}^{\times} \times \mathbb{Z}_{p}^{\times} \rrbracket$ and $T:=[1, \exp (p)]-1$. Then $\left\{\left(T_{i}\right)_{i \in I}, T\right\}$ forms a full set of parameters of the weight space $\mathcal{W}$.

- We denote by $\Lambda$ the complete group ring $\mathbb{Z}_{p} \llbracket \mathcal{O}_{p}^{\times} \times \mathbb{Z}_{p}^{\times} \rrbracket$, and put $\mathfrak{m}_{\Lambda}:=\left(p,\left(T_{i}\right)_{i \in I}\right) \subset$ $\Lambda$. For every character $\omega \in H^{\vee}$, let $\Lambda_{\omega}:=\Lambda \otimes_{\mathbb{Z}_{p}[H], \omega} \mathbb{Z}_{p}$. Under the above notations, we have $\Lambda_{\omega}=\mathbb{Z}_{p} \llbracket\left(T_{i}\right)_{i \in I}, T \rrbracket$.

- For a nonempty subset $J$ of $I$, we set $\Lambda_{J}:=\mathbb{Z}_{p} \llbracket \mathcal{O}_{p, J}^{\times} \times \mathbb{Z}_{p}^{\times} \rrbracket$. We use $H_{J} \subset H$ to denote the torsion subgroup of $\mathcal{O}_{p, J}^{\times} \times \mathbb{Z}_{p}^{\times}$. Under the isomorphism $\Lambda_{J} \cong \mathbb{Z}_{p}\left[H_{J}\right] \otimes_{\mathbb{Z}_{p}}$ 
$\mathbb{Z}_{p} \llbracket\left(T_{j}\right)_{j \in J}, T \rrbracket$, we set $\Lambda_{J}^{>1 / p}:=\mathbb{Z}_{p}\left[H_{J}\right] \otimes_{\mathbb{Z}_{p}} \mathbb{Z}_{p} \llbracket\left(T_{j}, \frac{p}{T_{j}}\right)_{j \in J}, T \rrbracket$ and $\mathfrak{m}_{\Lambda_{J}^{>1 / p}}$ be the ideal of $\Lambda_{J}^{>1 / p}$ generated by the elements $\left(T_{j}\right)_{j \in J}$. Note that since $p=T_{j} \cdot \frac{p}{T_{j}}$ in $\Lambda_{J}^{>1 / p}$, we have $p \in \mathfrak{m}_{\Lambda_{J}^{>1 / p}}$.

- When $J=I$, we write $\Lambda^{>1 / p}$ (resp. $\mathfrak{m}_{\Lambda}^{>1 / p}$ ) for $\Lambda_{J}^{>1 / p}$ (resp. $\mathfrak{m}_{\Lambda_{J}^{>1 / p}}$ ) for simplicity. In particular, we have $\mathfrak{m}_{\Lambda>1 / p}=\mathfrak{m}_{\Lambda} \cdot \Lambda^{>1 / p}$, and $\mathfrak{m}_{\Lambda>1 / p}$ is generated by $\left(T_{i}\right)_{i \in I}$ in $\Lambda^{>1 / p}$. We also have $p \in \mathfrak{m}_{\Lambda^{>1 / p}}$.

4.2. Explicit expression of $U_{\pi_{j}}$-operators on the space of $p$-adic automorphic forms. First we give an explicit expression of the $U_{\pi_{j}}$-operator on the space of (generalized) integral $p$-adic automorphic forms. This is a generalization of [20, Proposition 3.1].

Proposition 4.2.1. Let $J$ be a nonempty subset of $I$ and $\chi_{J}: \mathcal{O}_{p, J}^{\times} \times \mathbb{Z}_{p}^{\times} \rightarrow A^{\times}$be a continuous character. Fix $j \in J$. Under the isomorphism $S_{\kappa, J}^{D}\left(K^{p}, A\right) \stackrel{\cong}{\rightarrow} \bigoplus_{k=0}^{s-1} \mathcal{C}_{\chi_{J}}\left(\operatorname{Iw}_{\pi}, A\right)$, the $U_{\pi_{j}}$-operator on this space can be described by the following commutative diagram:

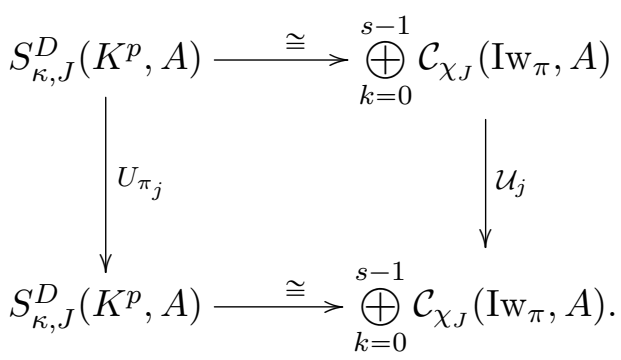

Here the right vertical map $\mathcal{U}_{j}$ in the above diagram is given by an $s \times s$ matrix with the following descriptions:

(1) Each entry of $\mathcal{U}_{j}$ is a sum of operators of the form $\circ \delta_{p}$, where $\delta_{p}=\left(\delta_{i}\right)_{i \in I} \in \mathrm{M}_{2}\left(\mathcal{O}_{p}\right)$ have the property that $\delta_{i} \in \mathrm{Iw}_{\pi_{i}}$ for all $i \neq j$, and $\delta_{j}$ belongs to $\left(\begin{array}{cc}\pi_{j} \mathcal{O}_{\mathfrak{p}_{j}} & \mathcal{O}_{\mathfrak{p}_{j}} \\ \pi_{j} \mathcal{O}_{\mathfrak{p}_{j}} & \mathcal{O}_{\mathfrak{p}_{j}}^{\times}\end{array}\right) \subset$ $M_{\pi, j}$, where $\circ \delta_{p}$ is the right action of the monoid $\mathbf{M}_{\pi, J} \times \mathrm{Iw}_{\pi, J^{c}}$ on the spaces $\mathcal{C}_{\chi_{J}}\left(\operatorname{Iw}_{\pi}, A\right)$ defined in $\$ 3.7$.

(2) There are exactly $p$ such operators appearing in each row and column of $\mathcal{U}_{j}$.

Proof. The proof is almost identical with that of [20, Proposition 3.1] and we only give a sketch here. For every $l=0, \ldots, p-1$ and $k=0, \ldots, s-1$, we can write $\gamma_{k} v_{j, l}^{-1}$ uniquely as $\delta_{l, k} \gamma_{\alpha_{l, k}} u_{l, k}$, for $\delta_{l, k} \in D^{\times}, \alpha_{l, k} \in\{0, \ldots, s-1\}$ and $u_{l, k} \in K^{p} \operatorname{Iw}_{\pi}$. Then

$$
\begin{aligned}
U_{\pi_{j}}(\phi)\left(\gamma_{k}\right) & =\sum_{l=0}^{p-1} \phi\left(\gamma_{\alpha_{l, k}} u_{l, k}\right) \circ v_{j, l}=\sum_{l=0}^{p-1}\left(\phi\left(\gamma_{\alpha_{l, k}}\right) \circ u_{l, k, p}\right) \circ v_{j, l} \\
& =\sum_{l=0}^{p-1} \phi\left(\gamma_{\alpha_{l, k}}\right) \circ\left(u_{l, k, p} v_{j, l}\right),
\end{aligned}
$$

where $u_{l, k, p} \in \mathrm{Iw}_{\pi}$ is the $\mathrm{GL}_{2}\left(F_{p}\right)$-component of $u_{l, k}$.

Let $\delta_{l, k, p}=u_{l, k, p} v_{j, l}$ for all $l$ 's and $k$ 's. It is straightforward to check that $\delta_{l, k, p}$ 's satisfy the stated property.

4.3. p-adic analysis. It follows from Proposition 4.2.1 that to study the $U_{\pi_{j}}$-operators for $j \in I$, it is crucial to understand the action of the monoid $\mathbf{M}_{\pi} \subset \mathrm{GL}_{2}\left(F_{p}\right)$ on the space $\mathcal{C}\left(\mathcal{O}_{p}, A\right)$. When $F=\mathbb{Q}$ (and hence $\left.\mathcal{O}_{p}=\mathbb{Z}_{p}\right)$ and $\kappa: \mathbb{Z}_{p}^{\times} \rightarrow \Lambda^{\times}=\left(\mathbb{Z}_{p} \llbracket \mathbb{Z}_{p}^{\times} \rrbracket\right)^{\times}$is the 
universal character, this question has been studied carefully by Liu-Wan-Xiao in [20, §3]. We will recall their results in this section and temporarily adopt their notations.

For $F=\mathbb{Q}$, the monoid $\mathbf{M}_{\pi}$ becomes $\left\{\left[\begin{array}{ll}a & b \\ c & d\end{array}\right] \in \mathrm{M}_{2}\left(\mathbb{Z}_{p}\right)|p| c, p \nmid d\right.$ and $\left.a d-b c \neq 0\right\}$. The Iwasawa algebra $\Lambda$ decomposes as $\mathbb{Z}_{p} \llbracket \mathbb{Z}_{p}^{\times} \rrbracket \cong \mathbb{Z}_{p}[\Delta] \otimes_{\mathbb{Z}_{p}} \mathbb{Z}_{p} \llbracket T \rrbracket$, where $\Delta \subset \mathbb{Z}_{p}^{\times}$is the torsion subgroup, $T=[\exp (p)]-1$. Denote $\mathfrak{m}_{\Lambda}:=(p, T) \subset \Lambda$. The space $\mathcal{C}\left(\mathbb{Z}_{p}, \Lambda\right)$ admits an orthonormal basis $\left\{e_{0}=1, e_{1}=z, e_{2}=\left(\begin{array}{c}z \\ 2\end{array}\right), \ldots\right\}$, which is called the Mahler basis (we refer $[20, \S 2.16]$ for more details). It also carries a right action of the monoid $\mathbf{M}_{\pi}$ defined by

$$
h \circ \delta(z):=\chi(c z+d) h\left(\frac{a z+b}{c z+d}\right) \text { for } h \in \mathcal{C}\left(\mathbb{Z}_{p}, \Lambda\right) \text { and } \delta \in \mathbf{M}_{\pi} .
$$

For $\delta_{p}=\left[\begin{array}{ll}a & b \\ c & d\end{array}\right] \in \mathbf{M}_{\pi}$, we use $P\left(\delta_{p}\right)=\left(P_{m, n}\left(\delta_{p}\right)\right)_{m, n \geqslant 0}$ to denote the infinite matrix for the action of $\delta_{p}$ on $\mathcal{C}\left(\mathbb{Z}_{p}, \Lambda\right)$ with respect to the Mahler basis, i.e. $P_{m, n}\left(\delta_{p}\right)$ is the coefficient of $\left(\begin{array}{c}z \\ m\end{array}\right)$ of the function $\left(\begin{array}{c}z \\ n\end{array}\right) \circ \delta_{p}$. Then we have the following estimation.

Proposition 4.3.1 ( [20], Proposition 3.24).

(1) When $\delta_{p}=\left[\begin{array}{ll}a & b \\ c & d\end{array}\right] \in\left[\begin{array}{ll}p \mathbb{Z}_{p} & \mathbb{Z}_{p} \\ p \mathbb{Z}_{p} & \mathbb{Z}_{p}^{\times}\end{array}\right]$, the coefficient $P_{m, n}\left(\delta_{p}\right)$ belongs to $\mathfrak{m}_{\Lambda}^{\max \left\{m-\left\lfloor\frac{n}{p}\right\rfloor, 0\right\}}$.

(2) When $\delta_{p}=\left[\begin{array}{ll}a & b \\ c & d\end{array}\right] \in \mathbf{M}_{\pi}$, the coefficient $P_{m, n}\left(\delta_{p}\right)$ belongs to $\mathfrak{m}_{\Lambda}^{\max \{m-n, 0\}}$.

4.4. Orthonormalizable spaces and compact operators. In this section we recall the notions of orthonormal basis and compact operator defined in [20, §5] and apply the theory to the spaces of (generalized) integral $p$-adic automorphic forms.

Definition 4.4.1. Let $R$ be a complete noetherian ring with ideal of definition $\mathfrak{m}_{R}$.

(1) A topological $R$-module $M$ is called orthonormalizable if it is isomorphic to the topological $R$-module:

$$
\hat{\oplus}_{i \in \mathbb{Z} \geqslant 0} R e_{i}:=\varliminf_{n}^{\lim }\left(\oplus_{i \in \mathbb{Z} \geqslant 0}\left(R / \mathfrak{m}_{R}^{n}\right) e_{i}\right) .
$$

More precisely, $R$ is orthonormalizable if there exists $\left\{e_{i} \mid i \in \mathbb{Z}_{\geqslant 0}\right\} \subset M$, such that every element $m$ in $M$ can be written uniquely as $m=\sum_{i \in \mathbb{Z}_{\geqslant 0}} a_{i} e_{i}$, with $a_{i} \in R$ and $\lim _{i \rightarrow \infty} a_{i}=0$ in $R$. The set $\left\{e_{i} \mid i \in \mathbb{Z}_{\geqslant 0}\right\}$ is called an orthonormal basis of $M$.

(2) Let $M$ be an orthonormalizable $R$-module. Let $U: M \rightarrow M$ be a continuous $R$ linear operator on $M$. $U$ is called compact if the induced operator on $M / \mathfrak{m}_{R}^{n} M$ has finitely generated image for all $n \in \mathbb{Z}_{\geqslant 0}$.

Keep the notations as in Definition 4.4.1. We fix an orthonormal basis $\left\{e_{m} \mid m \in \mathbb{Z}_{\geqslant 0}\right\}$ of $M$, and let $P \in \mathrm{M}_{\infty}(R)$ be the matrix associated to $U$ under this basis. We define the characteristic power series of the $U$-operator by $\operatorname{char}(U, M):=\operatorname{det}\left(\mathrm{I}_{\infty}-X P\right)=\lim _{n \rightarrow \infty} \operatorname{det}\left(\mathrm{I}_{\infty}-X(P\right.$ $\left.\left.\bmod \mathfrak{m}_{R}^{n}\right)\right) \in R \llbracket X \rrbracket$. The formal power series $\operatorname{char}(U, M)$ is well defined and it does not depend on the choice of the orthonormal basis. We will refer to [20, Definition 5.1] for more details.

We apply the above notions to the spaces of integral $p$-adic automorphic forms and their Hecke operators. Let $\chi^{\prime}: \mathcal{O}_{p}^{\times} \times \mathbb{Z}_{p}^{\times} \rightarrow\left(\Lambda^{>1 / p}\right)^{\times}$be the universal character and $\kappa^{\prime}: \mathcal{O}_{p}^{\times} \times \mathcal{O}_{p}^{\times} \rightarrow\left(\Lambda^{>1 / p}\right)^{\times}$be the associated character. Recall that we can identify the induced representation $\operatorname{Ind}_{B\left(\mathcal{O}_{p}\right)}^{\mathrm{Iw}_{\pi}}\left(\kappa_{B}^{\prime}\right)$ with the space $\mathcal{C}\left(\mathcal{O}_{p}, \Lambda^{>1 / p}\right)$. The latter space admits an orthonormal basis (as a topological $\Lambda^{>1 / p}$-module) $\left\{e_{m}=\prod_{i \in I}\left(\begin{array}{c}z_{i} \\ m_{i}\end{array}\right) \mid m=\left(m_{i}\right)_{i \in I} \in \mathbb{Z}_{\geqslant 0}^{I}\right\}$, 
where $z_{i}$ is the coordinate of the $i$ th component of $\mathcal{O}_{p}=\prod_{i \in I} \mathcal{O}_{\mathfrak{p}_{i}}$. Similar to [20], we consider a closed subspace $\mathcal{C}\left(\mathcal{O}_{p}, \Lambda^{>1 / p}\right)^{m o d}=\hat{\oplus}_{m \in \mathbb{Z}_{\geqslant 0}^{I}} \Lambda^{>1 / p} e_{m}^{\prime}$, where $e_{m}^{\prime}=\prod_{i \in I} T_{i}^{m_{i}}\left(\begin{array}{c}z_{i} \\ m_{i}\end{array}\right)$.

We claim that the space $\mathcal{C}\left(\mathcal{O}_{p}, \Lambda^{>1 / p}\right)^{\text {mod }}$ is stable under the action of the monoid $\mathbf{M}_{\pi}$. In fact, for any $i \in I$ and $\delta_{i} \in \mathbf{M}_{\pi_{i}}$, by Proposition 4.3.1 (2) and $p=T_{i} \cdot \frac{p}{T_{i}}$ in $\Lambda^{>1 / p}$, we have $\left(\begin{array}{c}z_{i} \\ m_{i}\end{array}\right) \circ \delta_{i}=\sum_{n_{i} \geqslant 0} a_{n_{i}, m_{i}}\left(\begin{array}{c}z_{i} \\ n_{i}\end{array}\right)$ with $a_{n_{i}, m_{i}} \in T_{i}^{\max \left\{n_{i}-m_{i}, 0\right\}} \Lambda^{>1 / p}$, and hence $\left(T_{i}^{m_{i}}\left(\begin{array}{c}z_{i} \\ m_{i}\end{array}\right)\right) \circ \delta_{i}=\sum_{n_{i} \geqslant 0} b_{n_{i}, m_{i}} T_{i}^{n_{i}}\left(\begin{array}{c}z_{i} \\ n_{i}\end{array}\right)$ with $b_{n_{i}, m_{i}}=T_{i}^{m_{i}-n_{i}} a_{n_{i}, m_{i}} \in \Lambda^{>1 / p}$. This implies that $\mathcal{C}\left(\mathcal{O}_{p}, \Lambda^{>1 / p}\right)^{\text {mod }}$ is stable under the action of $\mathbf{M}_{\pi_{i}}$. Combined with $\mathbf{M}_{\pi}=\prod_{i \in I} \mathbf{M}_{\pi_{i}}$, this proves our claim.

The space of integral 1-convergent automorphic forms are defined by $S_{\kappa, I}^{D, I}\left(K^{p}, \Lambda^{>1 / p}\right):=\left\{\phi: D^{\times} \backslash D_{f}^{\times} / K^{p} \rightarrow \mathcal{C}\left(\mathcal{O}_{p}, \Lambda^{>1 / p}\right)^{m o d} \mid \phi(x u)=\phi(x) \circ u\right.$, for all $\left.x \in D_{f}^{\times}, u \in \operatorname{Iw}_{\pi}\right\}$ We have an explicit expression of $S_{\kappa, I}^{D, I}\left(K^{p}\right)$ as before: $S_{\kappa, I}^{D, I}\left(K^{p}\right) \stackrel{\cong}{\rightarrow} \bigoplus_{k=0}^{s-1} \mathcal{C}\left(\mathcal{O}_{p}, \Lambda^{>1 / p}\right)^{m o d}$. Hence the topological $\Lambda^{>1 / p}$-module $S_{\kappa, I}^{D, I}\left(K^{p}\right)$ has an orthonormal basis $\left\{e_{k, m}^{\prime} \mid k=0, \ldots, s-\right.$ $\left.1, m \in \mathbb{Z}_{\geqslant 0}^{I}\right\}$, such that $\left\{e_{k, m}^{\prime} \mid m \in \mathbb{Z}_{\geqslant 0}^{I}\right\}$ is the orthonormal basis of the $k$-th direct summand in $\bigoplus_{k=0}^{s-1} \mathcal{C}\left(\mathcal{O}_{p}, \Lambda^{>1 / p}\right)^{\text {mod }}$ defined above, for every $k=0, \ldots, s-1$.

I claim that the $U_{\pi^{-}}$-operator on the space $S_{\kappa, I}^{D, I}\left(K^{p}\right)$ is compact. In fact, by Proposition 4.3.1 (1), for $\delta_{i} \in\left[\begin{array}{ll}\pi_{i} \mathcal{O}_{\mathfrak{p}_{i}} & \mathcal{O}_{\mathfrak{p}_{i}} \\ \pi_{i} \mathcal{O}_{\mathfrak{p}_{i}} & \mathcal{O}_{\mathfrak{p}_{i}}^{\times}\end{array}\right]$and $m_{i} \in \mathbb{Z}_{\geqslant 0}$, we have $\left(\begin{array}{c}z_{i} \\ m_{i}\end{array}\right) \circ \delta_{i}=\sum_{n_{i} \geqslant 0} a_{n_{i}, m_{i}}\left(\begin{array}{c}z_{i} \\ n_{i}\end{array}\right)$ with $a_{n_{i}, m_{i}} \in T_{i}^{\max \left\{n_{i}-\left\lfloor\frac{m_{i}}{p}\right\rfloor, 0\right\}} \Lambda^{>1 / p}$. Therefore $\left(T_{i}^{m_{i}}\left(\begin{array}{c}z_{i} \\ m_{i}\end{array}\right)\right) \circ \delta_{i}=\sum_{n_{i} \geqslant 0} b_{n_{i}, m_{i}} T_{i}^{n_{i}}\left(\begin{array}{c}z_{i} \\ n_{i}\end{array}\right)$, with $b_{n_{i}, m_{i}}=T_{i}^{m_{i}-n_{i}} a_{n_{i}, m_{i}} \in T_{i}^{m_{i}-\left\lfloor\frac{m_{i}}{p}\right\rfloor} \Lambda^{>1 / p} \subset \mathfrak{m}_{\Lambda^{>1 / p}}^{m_{i}-\left\lfloor\frac{m_{i}}{p}\right\rfloor}$. Hence for $\delta_{p}=\left(\delta_{i}\right) \in\left[\begin{array}{ll}\pi \mathcal{O}_{p} & \mathcal{O}_{p} \\ \pi \mathcal{O}_{p} & \mathcal{O}_{p}^{\times}\end{array}\right]$ and $m=\left(m_{i}\right) \in \mathbb{Z}_{\geqslant 0}^{I}$, we have

$$
e_{m}^{\prime} \circ \delta_{p}=\left(\prod_{i \in I} T_{i}^{m_{i}}\left(\begin{array}{c}
z_{i} \\
m_{i}
\end{array}\right)\right) \circ \delta_{p}=\prod_{i \in I}\left(T_{i}^{m_{i}}\left(\begin{array}{c}
z_{i} \\
m_{i}
\end{array}\right)\right) \circ \delta_{i}=\sum_{n \in \mathbb{Z}_{\geqslant 0}^{I}} b_{n, m} e_{n}^{\prime},
$$

with $b_{n, m} \in \mathfrak{m}_{\Lambda>1 / p}^{\lambda_{m}}$, where $\lambda_{m}=\sum_{i \in I}\left(m_{i}-\left\lfloor\frac{m_{i}}{p}\right\rfloor\right)$. It follows from the above estimation and the explicit expression of the $U_{\pi^{-}}$-operator on $S_{\kappa, I}^{D, I}\left(K^{p}\right)$ that $U_{\pi}$ is compact.

Remark 4.4.2. Fix a point $x \in \mathcal{W}^{>1 / p}\left(\mathbb{C}_{p}\right)$ which corresponds to a continuous homomorphism $\chi: \Lambda^{>1 / p} \rightarrow \mathbb{C}_{p}$. Let $V:=\mathcal{C}\left(\mathcal{O}_{p}, \Lambda^{>1 / p}\right)^{\text {mod }} \hat{\otimes}_{\Lambda>1 / p}, \chi \mathbb{C}_{p}$ be the specialization of $\mathcal{C}\left(\mathcal{O}_{p}, \Lambda^{>1 / p}\right)^{\text {mod }}$ at $x$. By 10, Theorem I.4.7], there exist $r<r^{\prime}$ in $\mathcal{N}^{I}$ (with the obvious partial order) that depend on the valuations $v_{p}\left(\chi\left(T_{i}\right)\right)^{\prime}$ 's for all $i \in I$ such that $\mathcal{A}_{\kappa, r} \subset V \subset \mathcal{A}_{\kappa, r^{\prime}}$, and hence $S_{\kappa}^{D}\left(K^{p} \mathrm{Iw}_{\pi}, r\right) \subset S_{\kappa, I}^{D, I}\left(K^{p}, \Lambda^{>1 / p}\right) \hat{\otimes}_{\Lambda^{>1 / p}, \chi} \mathbb{C}_{p} \subset S_{\kappa}^{D}\left(K^{p} \operatorname{Iw}_{\pi}, r^{\prime}\right)$. Therefore the space $S_{\kappa, I}^{D, I}\left(K^{p}, \Lambda^{>1 / p}\right) \hat{\otimes}_{\Lambda^{>1 / p}, \chi} \mathbb{C}_{p}$ contains all the finite $U_{\pi^{-}}$-slope systems of Hecke eigenvalues.

4.5. Continuous functions and distribution algebras. Let $G$ be a profinite group endowed with the profinite topology. Let $A$ be a $\mathbb{Z}_{p}$-algebra and $I$ be an ideal of $A$. We assume that $A$ is endowed with the $I$-adic topology and is complete under this topology. The typical example we are interested is that $A=\Lambda_{J}^{>1 / p}$ equipped with the $\mathfrak{m}_{\Lambda_{J}^{>1 / p}}$-adic topology 
for a nonempty subset $J$ of $I$. On the set $\mathcal{C}(G, A)$, we give it the uniform topology, i.e. for any $f \in \mathcal{C}(G, A)$, it has a basis $\left\{U_{n}\right\}$ of open neighborhoods as $U_{n}=\{g \in \mathcal{C}(G, A) \mid f(x)-g(x) \in$ $I^{n}$ for all $\left.x \in G\right\}$. Let $A \llbracket G \rrbracket:=\lim _{U \subset G} A[G / U]$ be the complete group ring of $G$ over $A$, where $U$ ranges over all the open normal subgroups of $G$. For each $U$, the free $A$-module $A[G / U]$ is endowed with the product topology and $A \llbracket G \rrbracket$ is endowed with the inverse limit topology. We use $\mathcal{D}(G, A)$ to denote the set $\operatorname{Hom}_{A}(\mathcal{C}(G, A), A)$ of continuous $A$-linear maps from $\mathcal{C}(G, A)$ to $A$ (here continuity follows from $A$-linearity). Then we have the following.

Proposition 4.5.1. There is a natural isomorphism of $A$-modules $A \llbracket G \rrbracket \cong \mathcal{D}(G, A)$. Under this isomorphism the topology on $A \llbracket G \rrbracket$ corresponds to the weak topology on $\mathcal{D}(G, A)$.

Proof. The proof is almost identical to the proof of [18, Lemma 3.1.3], which handles the case when $A$ is the unit ball of a Banach-Tate $\mathbb{Z}_{p}$-algebra. For completeness, we give a sketch of proof here.

For any nonempty open normal subgroup $U$ of $G$, we have an isomorphism of $A$ modules $A[G / U] \stackrel{\cong}{\rightrightarrows} \operatorname{Hom}_{A}(\mathcal{C}(G / U, A), A)$, which is also a homeomorphism. The projection $G \rightarrow G / U$ induces a natural $A$-module homomorphism $\mathcal{D}(G, A) \rightarrow \mathcal{D}(G / U, A)$ and hence a map $\mathcal{D}(G, A) \rightarrow \underset{U}{\lim } \mathcal{D}(G / U, A)$. If we use $\mathcal{C}_{s m}(G, A)$ to denote the $A$-submodule of $\mathcal{C}(G, A)$ consisting of locally constant functions, the map $\mathcal{D}(G, A) \rightarrow \underset{U}{\lim } \mathcal{D}(G / U, A) \cong A \llbracket G \rrbracket$ is the natural homomorphism $\operatorname{Hom}_{A}(\mathcal{C}(G, A), A) \rightarrow \operatorname{Hom}_{A}\left(\mathcal{C}_{s m}(G, A), A\right)$ induced by the inclusion map $\mathcal{C}_{s m}(G, A) \rightarrow \mathcal{C}(G, A)$. Since $\mathcal{C}_{s m}(G, A)$ is dense in $\mathcal{C}(G, A)$, it is clear that the map $\operatorname{Hom}_{A}(\mathcal{C}(G, A), A) \rightarrow \operatorname{Hom}_{A}\left(\mathcal{C}_{s m}(G, A), A\right)$ is an isomorphism of $A$-modules as well as a homeomorphism.

Remark 4.5.2. (1) We can also define a convolution product on $\mathcal{D}(G, A)$ so that the isomorphism in Proposition 4.5.1 is an isomorphism between $A$-algebras. But we do not need this fact and refer to [18, §3.1] to details (in a slightly different setting).

(2) For latter discussion, we recall a similar result as that of Proposition 4.5.1 discussed in [18, Proposition 3.1.4]. Let $A$ be a Banach-Tate $\mathbb{Z}_{p}$-algebra with unit ball $A_{0}$ and a multiplicative pseudo-uniformizer $\varpi$ in the sense of [18, Definition 2.1.2]. For a profinite group $G$, we define $A_{0} \llbracket G \rrbracket=\lim _{U \subset G} A_{0}[G / U]$ and $A \llbracket G \rrbracket=A_{0} \llbracket G \rrbracket\left[\frac{1}{\varpi}\right]$. It follows from [18, Proposition 3.1.4] that there is a natural $A$-Banach algebra isomorphism $A \llbracket G \rrbracket \cong \mathcal{D}(G, A)$. It restricts to an $A_{0}$-algebra isomorphism $A_{0} \llbracket G \rrbracket \cong$ $\mathcal{D}\left(G, A_{0}\right)$ that identifies the inverse star topology on the source with the weak star topology on the target. In particular, when $L$ is a closed subfield of $\mathbb{C}_{p}$, the $L$-Banach space $L \llbracket G \rrbracket$ is defined and isomorphic to $\mathcal{D}(G, L)$.

Let $H$ be a group or more generally a monoid. Suppose that we have a right action of $H$ on $\mathcal{C}(G, A)$. The above isomorphism induces a left action of $H$ on $A \llbracket G \rrbracket$ by requiring that $h \circ \mu(f)=\mu(f \circ h)$, for $h \in H, \mu \in \mathcal{D}(G, A)$ and $f \in \mathcal{C}(G, A)$.

We apply the above results to the spaces of (generalized) integral $p$-adic automorphic forms. Fix a nonempty subset $J$ of $I$. Recall that in $\$ 3.7$, we have an isomorphism $\mathcal{C}_{\chi_{J}}\left(\operatorname{Iw}_{\pi}, A\right) \cong \mathcal{C}\left(\mathcal{O}_{p, J} \times P_{J^{c}}, A\right)$. Combined with Proposition 4.5.1, it gives us an $A$-linear isomorphism $A \llbracket \mathcal{O}_{p, J} \times P_{J^{c}} \rrbracket \cong \operatorname{Hom}_{A}\left(\mathcal{C}_{\chi_{J}}\left(\operatorname{Iw}_{\pi}, A\right), A\right)$. In $\$ 3.7$, we have defined a right action of the monoid $\mathbf{M}_{\pi, J} \times \mathrm{Iw}_{\pi, J^{c}}$ on $\mathcal{C}_{\chi J}\left(\mathrm{Iw}_{\pi}, A\right)$. Therefore, we get a left action of $\mathbf{M}_{\pi, J} \times \operatorname{Iw}_{\pi, J^{c}}$ on $A \llbracket \mathcal{O}_{p, J} \times P_{J c} \rrbracket$. Under the isomorphism $A \llbracket \mathcal{O}_{p, J} \times P_{J c} \rrbracket \cong A \llbracket \mathcal{O}_{p, J} \rrbracket \hat{\otimes}_{A} A \llbracket P_{J^{c}} \rrbracket$, the monoid $\mathbf{M}_{\pi, J}$ acts on $A \llbracket \mathcal{O}_{p, J} \rrbracket$ and the group $\operatorname{Iw}_{\pi, J^{c}}$ acts on $A \llbracket P_{J c} \rrbracket$. We remark that the latter action has an explicit expression: for $u \in \mathrm{Iw}_{\pi, J^{c}}$ and $x \in A \llbracket P_{J^{c}} \rrbracket$, the left action of $u$ on $x$ is 
given by $u \circ x=\kappa_{B, J}\left(u_{2}^{*}\right) x \cdot u_{1}^{*}$, where $u_{1}^{*}\left(\operatorname{resp} . u_{2}^{*}\right)$ is the $P_{J^{c}}$-component $\left(\operatorname{resp} . D\left(\mathcal{O}_{p, J^{c}}\right)\right.$ component) of $u^{*} \in \mathrm{Iw}_{\pi, J^{c}}$ under the decomposition $\operatorname{Iw}_{\pi, J^{c}}=P_{J^{c}} \times D\left(\mathcal{O}_{p, J^{c}}\right), \kappa_{B, J}$ is the character defined in $\$ 3.7$, and $x \cdot u_{1}^{*}$ is the product in the complete group algebra $A \llbracket P_{J^{c}} \rrbracket$.

Convention 4.5.3. We always view the complete group algebra $A \llbracket P_{J^{c}} \rrbracket$ as a left $A \llbracket P_{J c}^{\prime} \rrbracket$ module induced by the left multiplication of $P_{J^{c}}^{\prime}$ on $P_{J^{c}}$. As $P_{J^{c}}^{\prime}$ is a normal subgroup of $P_{J^{c}}$ with quotient $P_{J^{c}} / P_{J^{c}}^{\prime} \cong \Delta_{J^{c}}, A \llbracket P_{J^{c}} \rrbracket$ is a free $A \llbracket P_{J^{c}}^{\prime} \rrbracket$ of rank $\left|\Delta_{J^{c}}\right|$. Under the isomorphism $A \llbracket \mathcal{O}_{p, J} \times P_{J^{c}} \rrbracket \cong A \llbracket \mathcal{O}_{p, J} \rrbracket \hat{\otimes}_{A} A \llbracket P_{J^{c}} \rrbracket$, the group algebra $A \llbracket \mathcal{O}_{p, J} \times P_{J^{c}} \rrbracket$ is also endowed with a left $A \llbracket P_{J^{c}}^{\prime} \rrbracket$-module structure. The left action of $\mathrm{Iw}_{\pi, J^{c}}$ on $A \llbracket P_{J^{c}}^{\prime} \rrbracket$ and $A \llbracket \mathcal{O}_{p, J} \times P_{J^{c}} \rrbracket$ defined above are $A \llbracket P_{J^{c}}^{\prime} \rrbracket$-linear. On the other hand, although $A \llbracket \mathcal{O}_{p, J} \rrbracket$ has a (commutative) ring structure, the left action of $\operatorname{Iw}_{\pi, J}$ on $A \llbracket \mathcal{O}_{p, J} \rrbracket$ defined above is not compatible with this ring structure. On the dual side, this is equivalent to the fact that the right action of $\operatorname{Iw}_{\pi, J}$ on the space $\mathcal{C}\left(\mathcal{O}_{p, J}, A\right)$ does not commute with the obvious translation action of $\mathcal{O}_{p, J}$ on $\mathcal{C}\left(\mathcal{O}_{p, J}, A\right)$. For this reason we only view $A \llbracket \mathcal{O}_{p, J} \times P_{J^{c}} \rrbracket$ as a left $A \llbracket P_{J^{c}}^{\prime} \rrbracket$-module in this paper.

Lemma 4.5.4. Let $M$ be a finitely generated right $\mathbb{Z}_{p} \llbracket \mathrm{Iw}_{\pi} \rrbracket$-module and $N$ be a right $A \llbracket \mathrm{Iw}_{\pi} \rrbracket$ module. Let $\langle\cdot, \cdot\rangle_{N}: N \times \operatorname{Hom}_{A}(N, A) \rightarrow A$ be the natural bilinear map. We endow $\operatorname{Hom}_{A}(N, A)$ with a left action of $\mathrm{Iw}_{\pi}$ as before, i.e. we have $\langle n \cdot g, l\rangle_{N}=\langle n, g \cdot l\rangle_{N}$ for all $n \in N, l \in \operatorname{Hom}_{A}(N, A)$ and $g \in \operatorname{Iw}_{\pi}$. Under the above notations, the map

$$
\begin{aligned}
M \times \operatorname{Hom}_{A}(N, A) & \rightarrow \operatorname{Hom}_{A}\left(\operatorname{Hom}_{\mathbb{Z}_{p} \llbracket \operatorname{Iw}_{\pi} \rrbracket}(M, N), A\right) \\
(m, l) & \mapsto F_{(m, l)},
\end{aligned}
$$

where $F_{(m, l)}: \operatorname{Hom}_{\mathbb{Z}_{p} \llbracket \operatorname{Iw}_{\pi} \rrbracket}(M, N) \rightarrow A$ is defined by $F_{(m, l)}(\varphi):=\langle\varphi(m), l\rangle_{N}$ for any $\varphi \in$ $\operatorname{Hom}_{\mathbb{Z}_{p} \llbracket \operatorname{Iw}_{\pi} \rrbracket}(M, N)$, induces an A-linear isomorphism

$$
\iota_{M, N}: M \hat{\otimes}_{\mathbb{Z}_{p} \llbracket \mathrm{Iw}_{\pi} \rrbracket} \operatorname{Hom}_{A}(N, A) \stackrel{\cong}{\longrightarrow} \operatorname{Hom}_{A}\left(\operatorname{Hom}_{\mathbb{Z}_{p} \llbracket \operatorname{Iw}_{\pi} \rrbracket}(M, N), A\right) .
$$

Proof. First we remark that since $M$ is a right $\mathbb{Z}_{p} \llbracket \mathrm{Iw}_{\pi} \rrbracket$ and $\operatorname{Hom}_{A}(N, A)$ carries a left $\operatorname{Iw}_{\pi^{-}}$ action, the completed tensor product $M \hat{\bigotimes}_{\mathbb{Z}_{p} \llbracket \operatorname{Iw}_{\pi} \rrbracket} \operatorname{Hom}_{A}(N, A)$ is meaningful. It is straightforward to verify that the map $M \times \operatorname{Hom}_{A}(N, A) \rightarrow \operatorname{Hom}_{A}\left(\operatorname{Hom}_{\mathbb{Z}_{p} \llbracket \operatorname{Iw}_{\pi} \rrbracket}(M, N), A\right)$ induces an $A$-linear homomorphism $\iota_{M, N}: M \hat{\otimes}_{\mathbb{Z}_{p} \llbracket \operatorname{Iw}_{\pi} \rrbracket} \operatorname{Hom}_{A}(N, A) \rightarrow \operatorname{Hom}_{A}\left(\operatorname{Hom}_{\mathbb{Z}_{p} \llbracket \operatorname{Iw}_{\pi} \rrbracket}(M, N), A\right)$ and $\iota_{M, N}$ is an isomorphism when $M$ is free. In the general case, we choose a resolution $F_{1} \rightarrow F_{0} \rightarrow M \rightarrow 0$ of $M$ with $F_{0}, F_{1}$ finite free $\mathbb{Z}_{p} \llbracket \mathrm{Iw}_{\pi} \rrbracket$-module. We apply the functors $M \rightarrow M \hat{\otimes}_{\mathbb{Z}_{p} \llbracket \operatorname{Iw}_{\pi} \rrbracket} \operatorname{Hom}_{A}(N, A)$ and $M \rightarrow \operatorname{Hom}_{A}\left(\operatorname{Hom}_{\mathbb{Z}_{p} \llbracket \operatorname{Iw}_{\pi} \rrbracket}(M, N), A\right)$ (with $N$ fixed) to the resolution $F_{1} \rightarrow F_{0} \rightarrow M \rightarrow 0$. A simple diagram chasing shows that $\iota_{M, N}$ is an isomorphism.

We denote by $S_{\kappa, I}^{D}\left(K^{p}, A\right)^{\vee}$ the $A$-linear dual of the space of $p$-adic automorphic forms $S_{\kappa, I}^{D}\left(K^{p}, A\right)$, and for any subset $J \subset I$ and a continuous character $\chi_{J}: \mathcal{O}_{p, J}^{\times} \times \mathbb{Z}_{p}^{\times} \rightarrow A^{\times}$, we define $S_{\kappa, J}^{D}\left(K^{p}, A\right)^{\vee}$ to be the $A$-linear dual of the space $S_{\kappa, J}^{D}\left(K^{p}, A\right)$. From the tautological isomorphism (3.6) and Lemma 4.5.4, we have the following expression of these spaces:

$$
S_{\kappa, I}^{D}\left(K^{p}, A\right)^{\vee}=\tilde{\mathrm{H}}_{0} \hat{\otimes}_{\mathbb{Z}_{p} \llbracket \mathrm{Iw}_{\pi} \rrbracket} A \llbracket \mathcal{O}_{p} \rrbracket \text { and } S_{\kappa, J}^{D}\left(K^{p}, A\right)^{\vee}=\tilde{\mathrm{H}}_{0} \hat{\otimes}_{\mathbb{Z}_{p} \llbracket \mathrm{Iw}_{\pi} \rrbracket} A \llbracket \mathcal{O}_{p, J} \times P_{J^{c}} \rrbracket,
$$

where $\tilde{\mathrm{H}}_{0}$ is the completed homology group defined in 3.7 .

We can translate the results we developed for the spaces $S_{\kappa, I}^{D}\left(K^{p}, A\right)$ and $S_{\kappa, J}^{D}\left(K^{p}, A\right)$ to their $A$-linear duals. We summarize these results as follows:

(1) For $j \in J$, we can define the $U_{\pi_{j}}$-operator on $S_{\kappa, I}^{D}\left(K^{p}, A\right)^{\vee}$ and $S_{\kappa, J}^{D}\left(K^{p}, A\right)^{\vee}$ by $U_{\pi_{j}}(h \hat{\otimes} \mu):=\sum_{l=0}^{p-1}\left(h \cdot v_{j, l}^{-1}\right) \hat{\otimes}\left(v_{j, l} \cdot \mu\right)$, for $h \hat{\otimes} \mu \in \tilde{\mathrm{H}}_{0} \hat{\otimes}_{\mathbb{Z}_{p} \llbracket \operatorname{Iw}_{\pi} \rrbracket} A \llbracket \mathcal{O}_{p} \rrbracket$ or $\tilde{\mathrm{H}}_{0} \hat{\otimes}_{\mathbb{Z}_{p} \llbracket \operatorname{Iw}_{\pi} \rrbracket} A \llbracket \mathcal{O}_{p, J} \times P_{J c} \rrbracket$. 
Under the natural $A$-bilinear pairing $\langle\cdot, \cdot\rangle: S_{\kappa, I}^{D}\left(K^{p}, A\right) \times S_{\kappa, I}^{D}\left(K^{p}, A\right)^{\vee} \rightarrow A$ (resp. $\left.\langle\cdot, \cdot\rangle_{J}: S_{\kappa, J}^{D}\left(K^{p}, A\right) \times S_{\kappa, J}^{D}\left(K^{p}, A\right)^{\vee} \rightarrow A\right)$, we have $\left\langle U_{\pi_{j}}(\phi), \psi\right\rangle=\left\langle\phi, U_{\pi_{j}}(\psi)\right\rangle$ (resp. $\left.\left\langle U_{\pi_{j}}(\phi), \psi\right\rangle_{J}=\left\langle\phi, U_{\pi_{j}}(\psi)\right\rangle_{J}\right)$.

(2) The right action of $B\left(\mathcal{O}_{p, J^{c}}\right)$ on $\mathcal{C}_{\chi_{J}}\left(\operatorname{Iw}_{\pi}, A\right)$ defined in Remark 3.7.10 induces a left action of $B\left(\mathcal{O}_{p, J^{c}}\right)$ on $A \llbracket \mathcal{O}_{p, J} \times P_{J^{c}} \rrbracket$. Moreover $A \llbracket \mathcal{O}_{p} \rrbracket$ can be identified with the coinvariant of $A \llbracket \mathcal{O}_{p, J} \times P_{J^{c}} \rrbracket$ under this action. For any two subsets $J_{1} \subset J_{2}$ of $I$, let $J_{3}=J_{2} \backslash J_{1}$. We have a natural surjective $A$-linear map $S_{\kappa, J_{1}}^{D}\left(K^{p}, A\right)^{\vee} \rightarrow$ $S_{\kappa, J_{2}}^{D}\left(K^{p}, A\right)^{\vee}$, which identifies the latter space with the $B\left(\mathcal{O}_{p, J_{3}}\right)$-coinvariants of the first space. Moreover, it is compatible with the $U_{\pi_{j}}$-operators on these two spaces for all $j \in J_{1}$.

(3) Let $\gamma_{k}$ for $k=0, \ldots, s-1$ be the elements of $D_{f}^{\times}$defined in 33.9 , which are viewed as elements in $\tilde{\mathrm{H}}_{0}$. By 4.1$)$, we write $S_{\kappa, I}^{D}\left(K^{p}, A\right)^{\vee}$ and $S_{\kappa, J}^{D}\left(\overline{K^{p}}, A\right)^{\vee}$ explicitly by

$$
\bigoplus_{k=0}^{s-1} A \llbracket \mathcal{O}_{p} \rrbracket \cong S_{\kappa, I}^{D}\left(K^{p}, A\right)^{\vee},\left(\mu_{k}\right)_{k=0, \ldots, s-1} \mapsto \sum_{k=0}^{k-1} \gamma_{k} \hat{\otimes} \mu_{k}
$$

and

$$
\bigoplus_{k=0}^{s-1} A \llbracket \mathcal{O}_{p, J} \times P_{J^{c}} \rrbracket \cong S_{\kappa, J}^{D}\left(K^{p}, A\right)^{\vee},\left(\mu_{k}\right)_{k=0, \ldots, s-1} \mapsto \sum_{k=0}^{k-1} \gamma_{k} \hat{\otimes} \mu_{k} .
$$

(4) The left $A \llbracket P_{J^{c}}^{\prime} \rrbracket$-module structure on $A \llbracket \mathcal{O}_{p, J} \times P_{J^{c}} \rrbracket$ defined in Convention 4.5 .3 induces a left $A \llbracket P_{J^{c}}^{\prime} \rrbracket$-module structure on the space $S_{\kappa, J}^{D}\left(K^{p}, A\right)^{\vee}$. The $U_{\pi_{j}}$-operator on $S_{\kappa, J}^{D}\left(K^{p}, A\right)^{\vee}$ is $A \llbracket P_{J^{c}}^{\prime} \rrbracket$-linear for all $j \in J$.

Recall that in $\$ 4.4$, we define a closed subspace $\mathcal{C}\left(\mathcal{O}_{p}, \Lambda^{>1 / p}\right)^{\bmod }$ of $\mathcal{C}\left(\mathcal{O}_{p}, \Lambda^{>1 / p}\right)$, which is closed under the action of the monoid $\mathbf{M}_{\pi}$. We will generalize this construction to define a closed subspace $\mathcal{C}\left(\mathcal{O}_{p, J} \times P_{J^{c}}, \Lambda_{J}^{>1 / p}\right)^{J^{\prime}-\bmod }$ of $\mathcal{C}\left(\mathcal{O}_{p, J} \times P_{J^{c}}, \Lambda_{J}^{>1 / p}\right)$ for any nonempty subsets $J^{\prime} \subset J$ of $I$. We cannot define this space directly as there is no Mahler basis for the space $\mathcal{C}\left(\mathcal{O}_{p, J} \times P_{J^{c}}, \Lambda_{J}^{>1 / p}\right)$. We will pass to the dual side to make the definition and then dual back.

First we explain the construction in the case $F=\mathbb{Q}$. Then $\mathcal{O}_{p}=\mathbb{Z}_{p}$ and we assume $\Lambda^{>1 / p}=\mathbb{Z}_{p} \llbracket T, \frac{p}{T} \rrbracket$. It follows from Amice transformation that the $\Lambda^{>1 / p}$-dual space of $\mathcal{O}\left(\mathbb{Z}_{p}, \Lambda^{>1 / p}\right)$ is isomorphic to $\Lambda^{>1 / p} \llbracket X \rrbracket$ and under this isomorphism, the dual basis of the Mahler basis $\left\{e_{m}=\left(\begin{array}{c}z \\ m\end{array}\right) \mid m \geqslant 0\right\}$ is given by $\left\{X^{m} \mid m \geqslant 0\right\}$. Combining it with Proposition 4.5.1, we have an isomorphism $\Lambda^{>1 / p} \llbracket \mathbb{Z}_{p} \rrbracket \cong \Lambda^{>1 / p} \llbracket X \rrbracket$, under which [1]-1 corresponds to $X$. We use $\Lambda^{>1 / p} \llbracket X \rrbracket^{\text {mod }}$ to denote the $\Lambda^{>1 / p}$-dual space of the closed subspace $\mathcal{C}\left(\mathbb{Z}_{p}, \Lambda^{>1 / p}\right)^{\text {mod }}$. Then as a $\Lambda^{>1 / p}$-module, it is isomorphic to $\Lambda^{>1 / p} \llbracket X^{\prime} \rrbracket$, and under this isomorphism the dual basis of the modified Mahler basis $\left\{e_{m}^{\prime}=T^{m}\left(\begin{array}{c}z \\ m\end{array}\right) \mid m \geqslant 0\right\}$ corresponds to $\left\{X^{\prime m} \mid m \geqslant 0\right\}$. The $\Lambda^{>1 / p}$-dual of the inclusion $\mathcal{C}\left(\mathbb{Z}_{p}, \Lambda^{>1 / p}\right)^{m o d} \rightarrow \mathcal{C}\left(\mathbb{Z}_{p}, \Lambda^{>1 / p}\right)$ is the $\Lambda^{>1 / p}$-algebra homomorphism $\Lambda^{>1 / p} \llbracket X \rrbracket \rightarrow \Lambda^{>1 / p} \llbracket X^{\prime} \rrbracket$ which sends $X$ to $T X^{\prime}$. Moreover, as the right action of the monoid $\mathbf{M}_{\pi}$ on $\mathcal{C}\left(\mathbb{Z}_{p}, \Lambda^{>1 / p}\right)$ keeps the subspace $\mathcal{C}\left(\mathbb{Z}_{p}, \Lambda^{>1 / p}\right)^{\text {mod }}$ stable, it induces a left action of $\mathbf{M}_{\pi}$ on $\Lambda^{>1 / p} \llbracket X^{\prime} \rrbracket$, and the map $\Lambda^{>1 / p} \llbracket X \rrbracket \rightarrow \Lambda^{>1 / p} \llbracket X^{\prime} \rrbracket$ defined above is compatible with the $\mathbf{M}_{\pi}$-actions on these two spaces.

Now we consider the general case. Fix two nonempty subsets $J^{\prime} \subset J$ of $I$. We define an isomorphism of $\Lambda_{J}^{>1 / p}$-algebras

$$
\iota_{J}: \Lambda_{J}^{>1 / p} \llbracket \mathcal{O}_{p, J} \rrbracket \rightarrow \Lambda_{J}^{>1 / p} \llbracket\left(X_{j}\right)_{j \in J} \rrbracket,\left[1_{j}\right]-1 \mapsto X_{j}
$$


for all $j \in J$, where $1_{j} \in \mathcal{O}_{p, J}=\prod_{j \in J} \mathcal{O}_{\mathfrak{p}_{j}}$ is the element with $j$-component 1 and $i$ component 0 for all $i \neq j$. We identify $\Lambda_{J}^{>1 / p} \llbracket \mathcal{O}_{p, J} \rrbracket$ with $\Lambda_{J}^{>1 / p} \llbracket\left(X_{j}\right)_{j \in J} \rrbracket$ via $\iota_{J}$. We put $\Lambda_{J}^{>1 / p} \llbracket \mathcal{O}_{p, J} \rrbracket^{J^{\prime}-\text { mod }}:=\Lambda_{J}^{>1 / p} \llbracket\left(X_{j}^{\prime}\right)_{j \in J^{\prime}},\left(X_{j}\right)_{j \in J \backslash J^{\prime}} \rrbracket$ and define an embedding of $\Lambda_{J}^{>1 / p}$ algebras $\Lambda_{J}^{>1 / p} \llbracket \mathcal{O}_{p, J} \rrbracket \rightarrow \Lambda_{J}^{>1 / p} \llbracket \mathcal{O}_{p, J} \rrbracket^{J^{\prime}-\bmod }$ by $X_{j} \mapsto T_{j} X_{j}^{\prime}$ for $j \in J^{\prime}$ and $X_{j} \mapsto X_{j}$ for $j \in J \backslash J^{\prime}$. Then we put $\Lambda_{J}^{>1 / p} \llbracket \mathcal{O}_{p, J} \times P_{J^{c}} \rrbracket^{J^{\prime}-\bmod }:=\Lambda_{J}^{>1 / p} \llbracket \mathcal{O}_{p, J} \rrbracket^{J^{\prime}-\bmod } \hat{\otimes}_{\Lambda_{J}^{>1 / p}} \Lambda_{J}^{>1 / p} \llbracket P_{J^{c}} \rrbracket$. Under the isomorphism $\mathcal{O}_{\mathfrak{p}_{j}} \cong \mathbb{Z}_{p}$, we have $\Lambda_{J}^{>1 / p} \llbracket \mathcal{O}_{\mathfrak{p}_{j}} \rrbracket^{j-\bmod } \cong \Lambda_{J}^{>1 / p} \llbracket \mathbb{Z}_{p} \rrbracket^{\text {mod }}$ defined above and hence we can endow $\Lambda_{J}^{>1 / p} \llbracket \mathcal{O}_{\mathfrak{p}_{j}} \rrbracket^{j-\bmod }$ a left action of the monoid $\mathbf{M}_{\pi_{j}}$. Under the isomorphism $\Lambda_{J}^{>1 / p} \llbracket \mathcal{O}_{p, J} \rrbracket^{J^{\prime}-\bmod } \cong\left(\hat{\otimes}_{j \in J^{\prime}} \Lambda_{J}^{>1 / p} \llbracket \mathcal{O}_{\mathfrak{p}_{j}} \rrbracket^{j-m o d}\right) \hat{\otimes}_{\Lambda_{J}^{>1 / p}} \Lambda_{J}^{>1 / p} \llbracket \mathcal{O}_{p, J \backslash J^{\prime}} \rrbracket$, we get a left action of the monoid $\mathbf{M}_{\pi, J}$ on $\Lambda_{J}^{>1 / p} \llbracket \mathcal{O}_{p, J} \rrbracket^{J^{\prime}-\text { mod }}$. Then the embedding $\Lambda_{J}^{>1 / p} \llbracket \mathcal{O}_{p, J} \rrbracket \hookrightarrow \Lambda_{J}^{>1 / p} \llbracket \mathcal{O}_{p, J} \rrbracket^{J^{\prime}-\bmod }\left(\operatorname{resp} . \quad \Lambda_{J}^{>1 / p} \llbracket \mathcal{O}_{p, J} \times P_{J^{c}} \rrbracket \rightarrow \Lambda_{J}^{>1 / p} \llbracket \mathcal{O}_{p, J} \times P_{J^{c}} \rrbracket^{J^{\prime}-\text { mod }}\right)$ is equivariant under the actions of the monoid $\mathbf{M}_{\pi, J}\left(\operatorname{resp} . \mathbf{M}_{\pi, J} \times \operatorname{Iw}_{\pi, J^{c}}\right)$ on these two spaces.

Define $\mathcal{C}_{\chi_{J}}\left(\operatorname{Iw}_{\pi}, \Lambda_{J}^{>1 / p}\right)^{J^{\prime}-\bmod }$ to be the $\Lambda_{J}^{>1 / p}$-dual space of $\Lambda_{J}^{>1 / p} \llbracket \mathcal{O}_{p, J} \times P_{J^{c}} \rrbracket^{J^{\prime}-\text { mod }}$, which carries a right action of the monoid $\mathbf{M}_{\pi, J} \times \mathrm{Iw}_{\pi, J^{c}}$. Similar with the definition of $S_{\kappa, I}^{D, I}\left(K^{p}, \Lambda_{J}^{>1 / p}\right)$, we set

$$
\begin{aligned}
& S_{\kappa, J}^{D, J^{\prime}}\left(K^{p}, \Lambda_{J}^{>1 / p}\right) \\
:= & \left\{\phi: D^{\times} \backslash D_{f}^{\times} / K^{p} \rightarrow \mathcal{C}_{\chi_{J}}\left(\operatorname{Iw}_{\pi}, \Lambda_{J}^{>1 / p}\right)^{J^{\prime}-\bmod } \mid \phi(x u)=\phi(x) \circ u \text { for all } x \in D_{f}^{\times}, u \in \operatorname{Iw}_{\pi}\right\},
\end{aligned}
$$

and $S_{\kappa, J}^{D, J^{\prime}}\left(K^{p}, \Lambda_{J}^{>1 / p}\right)^{\vee}$ to be its $\Lambda_{J}^{>1 / p}$-linear dual. Hence, we have

$$
S_{\kappa, J}^{D, J^{\prime}}\left(K^{p}, \Lambda_{J}^{>1 / p}\right)^{\vee}=\tilde{\mathrm{H}}_{0} \hat{\otimes}_{\mathbb{Z}_{p} \llbracket \operatorname{Iw}_{\pi} \rrbracket} \Lambda_{J}^{>1 / p} \llbracket \mathcal{O}_{p, J} \times P_{J^{c}} \rrbracket^{J^{\prime}-\bmod } .
$$

Remark 4.5.5. (1) Since our notation above is somewhat complicated, we make a remark to explain its meaning. In the notation $S_{\kappa, J}^{D, J^{\prime}}\left(K^{p}, \Lambda_{J}^{>1 / p}\right)$, the subscript $J$ under $S$ means that we consider generalized $p$-adic automorphic forms which behave like automorphic forms at places in $J$ and behave like dual of completed homology groups at places in $I \backslash J$. The superscript $J^{\prime}$ means that we 'modify' the basis at places in $J^{\prime}$, or more precisely, we consider the closed subspace $\mathcal{C}\left(\mathcal{O}_{p, J} \times P_{J^{c}}, \Lambda_{J}^{>1 / p}\right)^{J^{\prime}-\bmod }$ of $\mathcal{C}\left(\mathcal{O}_{p, J} \times P_{J^{c}}, \Lambda_{J}^{>1 / p}\right)$ defined before. In particular, we always have $J^{\prime} \subset J$ when writing $S_{\kappa, I}^{D, J^{\prime}}\left(K^{p}, \Lambda_{J}^{>1 / p}\right)$, and if $J^{\prime \prime} \subset J^{\prime} \subset J$ are three nonempty subsets of $I$, we have a natural map $S_{\kappa, J}^{D, J^{\prime}}\left(K^{p}, \Lambda_{J}^{>1 / p}\right) \rightarrow S_{\kappa, J}^{D, J^{\prime \prime}}\left(K^{p}, \Lambda_{J}^{>1 / p}\right)$, which is equivariant under the $U_{\pi_{j}}$-operator, for all $j \in J$.

(2) Fix a nonempty subset $J$ of $I$ and let $A=\Lambda_{J}$ or $\Lambda_{J}^{>1 / p}$. For $j \in J$, under the isomorphism $\mathbb{Z}_{p} \cong \mathcal{O}_{\mathfrak{p}_{j}}$, we can identify $\mathcal{C}\left(\mathbb{Z}_{p}, A\right)$ with $\mathcal{C}\left(\mathcal{O}_{\mathfrak{p}_{j}}, A\right)$. So it is meaningful to talk about the Mahler basis (resp. modified Mahler basis) of the space $\mathcal{C}\left(\mathcal{O}_{\mathfrak{p}_{j}}, A\right)$ (resp. $\left.\mathcal{C}\left(\mathcal{O}_{\mathfrak{p}_{j}}, A\right)^{\text {mod }}\right)$. We define the Mahler basis of $A \llbracket \mathcal{O}_{\mathfrak{p}_{j}} \rrbracket$ to be the dual basis of the Mahler basis of $\mathcal{C}\left(\mathcal{O}_{\mathfrak{p}_{j}}, A\right)$. Under the isomorphism $A \llbracket \mathcal{O}_{\mathfrak{p}_{j}} \rrbracket \cong A \llbracket X_{j} \rrbracket$ constructed as above, the Mahler basis of $A \llbracket \mathcal{O}_{\mathfrak{p}_{j}} \rrbracket$ is given by $\left\{X_{j}^{m} \mid m \geqslant 0\right\}$. In general, from the expression $A \llbracket \mathcal{O}_{p, J} \rrbracket=\hat{\otimes}_{j \in J} A \llbracket \mathcal{O}_{\mathfrak{p}_{j}} \rrbracket$, we can talk about the Mahler basis of $A \llbracket \mathcal{O}_{p, J} \rrbracket$. Under the isomorphism $A \llbracket \mathcal{O}_{p, J} \rrbracket \cong A \llbracket\left(X_{j}\right)_{j \in J} \rrbracket$, the Mahler basis is given by $\left\{\prod_{j \in J} X_{j}^{n_{j}} \mid n_{j} \geqslant 0\right\}$. Since $A \llbracket \mathcal{O}_{p, J} \times P_{J^{c}}^{\prime} \rrbracket=A \llbracket \mathcal{O}_{p, J} \rrbracket \hat{\otimes}_{A} A \llbracket P_{J^{c}}^{\prime} \rrbracket$, the Mahler basis 
of $A \llbracket \mathcal{O}_{p, J} \rrbracket$ becomes a basis of the left $A \llbracket P_{J^{c}}^{\prime} \rrbracket$-module $A \llbracket \mathcal{O}_{p, J} \times P_{J^{c}}^{\prime} \rrbracket$, which is called the Mahler basis of $A \llbracket \mathcal{O}_{p, J} \times P_{J^{c}}^{\prime} \rrbracket$. We make similar definitions for the modified Mahler basis of the spaces $A \llbracket \mathcal{O}_{\mathfrak{p}_{j}} \rrbracket^{\text {mod }}, A \llbracket \mathcal{O}_{p, J} \rrbracket^{J^{\prime}-\bmod }$ and $A \llbracket \mathcal{O}_{p, J} \times P_{J^{c}}^{\prime} \rrbracket^{J^{\prime}-\text { mod }}$.

(3) Strictly speaking, it is crucial for us to work with the $\Lambda_{J}^{>1 / p}$-dual spaces of $\mathcal{C}\left(\mathcal{O}_{p, J} \times\right.$ $\left.P_{J^{c}}, \Lambda_{J}^{>1 / p}\right)$ and $\mathcal{C}\left(\mathcal{O}_{p, J} \times P_{J^{c}}, \Lambda_{J}^{>1 / p}\right)^{J^{\prime}-\bmod }$. To explain the problem, we assume $J=I$ for simplicity. Recall that $\mathcal{C}\left(\mathcal{O}_{p}, \Lambda^{>1 / p}\right)^{\text {mod }}$ is the subspace of $\mathcal{C}\left(\mathcal{O}_{p}, \Lambda^{>1 / p}\right)$ spanned by the orthonormal basis $\left\{e_{m}^{\prime} \mid m \in \mathbb{Z}_{\geqslant 0}^{I}\right\}$, i.e. any element in $\mathcal{C}\left(\mathcal{O}_{p}, \Lambda^{>1 / p}\right)^{\text {mod }}$ is of the form $\sum_{m \in \mathbb{Z}_{\geqslant 0}^{I}} a_{m} e_{m}^{\prime}$ with $a_{m} \in \Lambda^{>1 / p}$ and $a_{m} \rightarrow 0$ as $|m|:=\sum_{i \in I} m_{i} \rightarrow \infty$ under the $\mathfrak{m}_{\Lambda>1 / p}$-adic topology. In particular, $\mathcal{C}\left(\mathcal{O}_{p}, \Lambda^{>1 / p}\right)^{\text {mod }}$ is not isomorphic to the direct product $\prod_{m \in \mathbb{Z}_{\geqslant 0}^{I}} \Lambda^{>1 / p}$, while its $\Lambda^{>1 / p}$-dual is. The computation in $\$ 4$ actually shows that there is a well-defined action of the monoid $\mathbf{M}_{\pi}$ on $\Lambda^{>1 / p} \llbracket \mathcal{O}_{p} \rrbracket^{I-m o d}$, such that the inclusion $\Lambda^{>1 / p} \llbracket \mathcal{O}_{p} \rrbracket \rightarrow \Lambda^{>1 / p} \llbracket \mathcal{O}_{p} \rrbracket^{I-m o d}$ is $\mathbf{M}_{\pi}$-equivariant. Hence the spaces $\Lambda^{>1 / p} \llbracket \mathcal{O}_{p, J} \times P_{J^{c}} \rrbracket^{J^{\prime}-m o d}$ for various subsets $J^{\prime} \subset J \subset I$ are the correct objects to consider. Since $S_{\kappa, I}^{D}\left(K^{p}, \Lambda^{>1 / p}\right)$ and its dual space $S_{\kappa, I}^{D}\left(K^{p}, \Lambda^{>1 / p}\right)^{\vee}$ share the same Hecke eigensystem, we can work with the space $S_{\kappa, I}^{D}\left(K^{p}, \Lambda^{>1 / p}\right)^{\vee}$ and embedded into the space $S_{\kappa, I}^{D, I}\left(K^{p}, \Lambda^{>1 / p}\right)^{\vee}$ to study the $U_{\pi_{i}}$-eigenvalues. In the rest of our proof of Theorem 1.0.2, we will insist to work with the dual spaces $S_{\kappa, J}^{D, J^{\prime}}\left(K^{p}, \Lambda_{J}^{>1 / p}\right)^{\vee}$ of generalized $p$-adic automorphic forms.

4.6. A filtration on the space of $p$-adic automorphic forms with respect to a $U_{\pi_{i}}$-operator. Throughout this section, we take $J=\{j\} \subset I$ consisting of a single place $j$ in $I$, and we use $R$ to denote the ring $\Lambda_{J}^{>1 / p}=\mathbb{Z}_{p}\left[H_{J}\right] \otimes_{\mathbb{Z}_{p}} \mathbb{Z}_{p} \llbracket T_{j}, \frac{p}{T_{j}}, T \rrbracket$. In particular we have $\mathcal{O}_{p, J}=\mathcal{O}_{\mathfrak{p}_{j}}$.

Let $\chi_{J}: \mathcal{O}_{p, J}^{\times} \times \mathbb{Z}_{p}^{\times}=\mathcal{O}_{\mathfrak{p}_{j}}^{\times} \times \mathbb{Z}_{p}^{\times} \rightarrow R^{\times}$be the universal character. Recall that the space $S_{\kappa, J}^{D}\left(K^{p}, R\right)^{\vee}$ is endowed with a left $R \llbracket P_{J^{c}}^{\prime} \rrbracket$-module structure. Moreover, the $U_{\pi_{j}}{ }^{-}$ operator and the explicit expression $S_{\kappa, J}^{D}\left(K^{p}, R\right)^{\vee} \cong \underset{k=0}{\stackrel{s-1}{\rightrightarrows}} R \llbracket \mathcal{O}_{\mathfrak{p}_{j}} \times P_{J^{c}} \rrbracket$ are both $R \llbracket P_{J^{c}}^{\prime} \rrbracket$ linear. We have a 'dual' result of Proposition 4.2.1. under the isomorphism $S_{\kappa, J}^{D}\left(K^{p}, R\right)^{\vee} \cong$ $\bigoplus_{k=0}^{s-1} R \llbracket \mathcal{O}_{\mathfrak{p}_{j}} \times P_{J^{c}} \rrbracket$, the $U_{\pi_{j}}$-operator can be described by the following commutative diagram: $k=0$

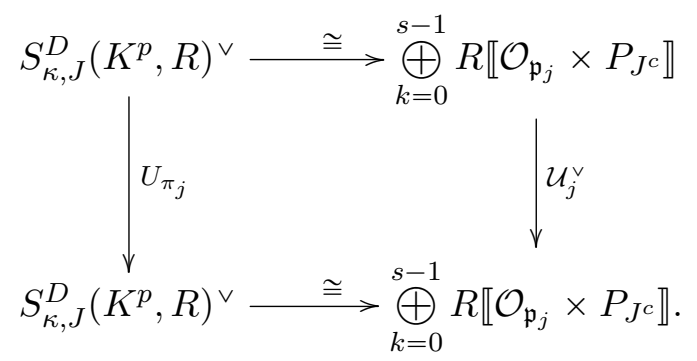

Here the right vertical maps $\mathcal{U}_{j}^{\vee}$ is the 'transpose' of the map $\mathcal{U}_{j}$ in Proposition 4.2.1. More explicitly, $\mathcal{U}_{j}^{\vee}$ is given by a $t \times t$ matrix with the following descriptions:

(1) Each entry of $\mathcal{U}_{j}^{\vee}$ is a sum of operators of the form $\delta_{p}$, where $\delta_{p}=\left(\delta_{i}\right)_{i \in I} \in \mathrm{M}_{2}\left(\mathcal{O}_{p}\right)$ have the same property as in Proposition 4.2.1.

(2) There are exactly $p$ such operators appearing in each row and column of $\mathcal{U}_{j}^{\vee}$. 
Notice that $R \llbracket P_{J^{c}} \rrbracket$ is a free left $R \llbracket P_{J^{c}}^{\prime} \rrbracket$-module of rank $\left|\Delta_{J^{c}}\right|$. If we fix a set of representatives in $P_{J^{c}}$ of the quotient $P_{J^{c}} / P_{J^{c}}^{\prime} \cong \Delta_{J^{c}}$, we obtain an $R \llbracket P_{J^{c}}^{\prime} \rrbracket$-linear isomorphism $\llbracket P_{J^{c}} \rrbracket \cong \bigoplus_{k=0}^{\left|\Delta_{J^{c}}\right|} R \llbracket P_{J^{c}}^{\prime} \rrbracket$. This induces an isomorphism $R \llbracket \mathcal{O}_{\mathfrak{p}_{j}} \times P_{J^{c}} \rrbracket \cong \bigoplus_{k=1}^{\left|\Delta_{J^{c}}\right|} R \llbracket \mathcal{O}_{\mathfrak{p}_{j}} \times P_{J^{c}}^{\prime} \rrbracket$ and we have an isomorphism $S_{\kappa, J}^{D}\left(K^{p}, R\right)^{\vee} \cong \underset{k=1}{\stackrel{S}{\rightrightarrows}} R \llbracket \mathcal{O}_{\mathfrak{p}_{j}} \times P_{J^{c}}^{\prime} \rrbracket$ of left $R \llbracket P_{J^{c}}^{\prime} \rrbracket$-modules, where $S=s \cdot\left|\Delta_{J^{c}}\right|$. Similarly, we have an $R \llbracket P_{J^{c}}^{\prime} \rrbracket$-linear isomorphism $S_{\kappa, J}^{D, J}\left(K^{p}, R\right)^{\vee} \cong$ $\stackrel{S}{\oplus} R \llbracket \mathcal{O}_{\mathfrak{p}_{j}} \times P_{J^{c}}^{\prime} \rrbracket^{J-m o d}$.

$k=1$

Under the above isomorphisms, we choose a basis $\left\{f_{k, m} \mid 1 \leqslant k \leqslant S, m \in \mathbb{Z}_{\geqslant 0}\right\}$ of $S_{\kappa, J}^{D}\left(K^{p}, R\right)^{\vee}$ as a left $R \llbracket P_{J^{c}}^{\prime} \rrbracket$-module, such that $\left\{f_{k, m} \mid m \in \mathbb{Z}_{\geqslant 0}\right\}$ is the Mahler basis of the $k$-th direct factor of $\oplus_{k=0}^{s-1} R \llbracket \mathcal{O}_{\mathfrak{p}_{j}} \times P_{J^{c}}^{\prime} \rrbracket$ for all $1 \leqslant k \leqslant S$. We choose a basis $\left\{f_{k, m}^{\text {mod }} \mid 1 \leqslant k \leqslant S, m \in \mathbb{Z}_{\geqslant 0}\right\}$ of $S_{\kappa, J}^{D, J}\left(K^{p}, R\right)^{\vee}$ in a similar way.

Notice that for any $\delta_{i} \in \mathbf{M}_{\pi_{i}}$, if we use $P\left(\delta_{i}\right)=\left(P_{m, n}\left(\delta_{i}\right)\right)_{m, n \geqslant 0}$ to denote the infinite matrix for the action of $\delta_{i}$ on $\mathcal{C}\left(\mathcal{O}_{\mathfrak{p}_{i}}, A\right)$ under the Mahler basis, then the matrix for the action of $\delta_{i}$ on $A \llbracket \mathcal{O}_{\mathfrak{p}_{i}} \rrbracket$ under the Mahler basis is the transpose of the matrix $P\left(\delta_{i}\right)$.

We use $N=\left(N_{m, n}\right)_{m, n \geqslant 0}\left(\operatorname{resp} . M=\left(M_{m, n}\right)_{m, n \geqslant 0}\right)$ to denote the matrix in $\mathrm{M}_{\infty}\left(R \llbracket P_{J^{c}}^{\prime} \rrbracket\right)$ which corresponds to the $U_{\pi_{j}}$-operator on $S_{\kappa, J}^{D}\left(K^{p}, R\right)^{\vee}\left(\operatorname{resp} . S_{\kappa, J}^{D, J}\left(K^{p}, R\right)^{\vee}\right)$ under the basis we choose above. It follows from Propositions 4.2.1, 4.3.1 and the above remark that we have $N_{m, n} \in\left(T_{j}\right)^{\max \left\{\left\lfloor\frac{n}{S}\right\rfloor-\left\lfloor\frac{m}{p S}\right\rfloor, 0\right\}}$. On the other hand, from the construction of the Mahler basis, the matrix $M$ is the conjugation of $N$ by the infinite diagonal matrix with diagonal entries $\underbrace{1,1, \ldots, 1}_{S}, \underbrace{T_{j}, T_{j}, \ldots, T_{j}}_{S}, \underbrace{T_{j}^{2}, T_{j}^{2}, \ldots, T_{j}^{2}}_{S}, \ldots$ Define two sequences $\underline{\lambda}, \underline{\mu}$ of integers as $\lambda_{n}=\left\lfloor\frac{n}{S}\right\rfloor-\left\lfloor\frac{n}{p S}\right\rfloor$ and $\mu_{0}=0, \mu_{n+1}-\mu_{n}=\lambda_{n}$ for all $n \in \mathbb{Z}_{\geqslant 0}$. The above computation implies that the matrix $M \in \mathrm{M}_{\infty}\left(R \llbracket P_{J^{c}}^{\prime} \rrbracket\right)$ is $\underline{\lambda}$-Hodge bounded with respect to the element $T_{j} \in R \subset R \llbracket P_{J^{c}}^{\prime} \rrbracket$.

In the following discussion, we fix a character $\omega=\left(\eta_{j}, \eta\right): H_{J}=\Delta_{j} \times \Delta \rightarrow \mathbb{Z}_{p}^{\times}$. Denote $R_{\omega_{J}}=R \otimes_{\mathbb{Z}_{p}\left[H_{J}\right], \omega_{J}} \mathbb{Z}_{p}$, which is naturally isomorphic to $\mathbb{Z}_{p} \llbracket T_{j}, \frac{p}{T_{j}}, T \rrbracket$. We construct a chain of ring homomorphisms as follows:

- Let $R \rightarrow R_{\omega_{J}}$ and $R \llbracket P_{J^{c}}^{\prime} \rrbracket \rightarrow R_{\omega_{J}} \llbracket P_{J^{c}}^{\prime} \rrbracket$ be the natural homomorphisms.

- Let $R_{\omega_{J}} \llbracket P_{J^{c}}^{\prime} \rrbracket \rightarrow R_{\omega_{J}}$ be the reduction map modulo the augmentation ideal of the complete group algebra $R_{\omega_{J}} \llbracket P_{J^{c}}^{\prime} \rrbracket$.

- Under the isomorphism $R_{\omega_{J}} \cong \mathbb{Z}_{p} \llbracket T_{j}, \frac{p}{T_{j}}, T \rrbracket$, let $R_{\omega_{J}} \rightarrow R_{j}:=\mathbb{Z}_{p} \llbracket T_{j}, \frac{p}{T_{j}} \rrbracket$ be the reduction map modulo the ideal generated by $T$.

- Let $R_{j} \rightarrow \mathbb{F}_{p} \llbracket T_{j} \rrbracket$ be the reduction map modulo the ideal generated by $\frac{p}{T_{j}}$.

We apply the above homomorphisms $R \llbracket P_{J^{c}}^{\prime} \rrbracket \rightarrow R_{\omega_{J}} \llbracket P_{J^{c}}^{\prime} \rrbracket \rightarrow R_{\omega_{J}} \rightarrow R_{j} \rightarrow \mathbb{F}_{p} \llbracket T_{j} \rrbracket$ to entries of the matrix $M$, and obtain matrices $M_{R_{\omega_{J}} \llbracket P_{J}^{\prime} \rrbracket} \in \mathrm{M}_{\infty}\left(R_{\omega_{J}} \llbracket P_{J^{c}}^{\prime} \rrbracket\right), M_{R_{\omega_{J}}} \in \mathrm{M}_{\infty}\left(R_{\omega_{J}}\right)$, $M_{R_{j}} \in \mathrm{M}_{\infty}\left(R_{j}\right)$ and $\bar{M} \in \mathrm{M}_{\infty}\left(\mathbb{F}_{p} \llbracket T_{j} \rrbracket\right)$. All these matrices are $\underline{\lambda}$-Hodge bounded with respect to the element $T_{j}$ as the matrix $M$ is so.

Fix $l \in 2 \mathbb{Z}_{\geqslant 0}$. For every character $\omega_{J^{c}}: \Delta_{J^{c}} \rightarrow \mathbb{Z}_{p}^{\times}$, we obtain a character $\omega=\left(\omega_{J}, \omega_{J^{c}}\right)$ : $H \rightarrow \mathbb{Z}_{p}^{\times}$. We construct a point $\chi_{l} \in \mathcal{W}\left(\mathbb{C}_{p}\right)$ whose associated character $\kappa_{l}: \mathcal{O}_{p}^{\times} \times \mathcal{O}_{p}^{\times} \rightarrow \mathbb{C}_{p}^{\times}$ is locally algebraic and corresponds to the triple $\left(n \in \mathbb{Z}_{\geqslant 0}^{I}, \nu \in \mathbb{Z}^{I}, \psi=\left(\psi_{1}, \psi_{2}\right)\right)$ defined as follows.

- Define $\nu:=\left(\nu_{i}\right)_{i \in I}$ by $\nu_{j}:=-\frac{l}{2}$ and $\nu_{i}:=0$ for all $i \neq j$, and set $n:=-2 \nu \in \mathbb{Z}_{\geqslant 0}^{I}$.

- Define two finite characters $\psi_{1}, \psi_{2}: \mathcal{O}_{p}^{\times} \rightarrow \mathbb{C}_{p}^{\times}$with the following properties: 
$-\left.\psi_{1}\right|_{1+\pi_{i} \mathcal{O}_{\mathfrak{p}_{i}}}$ and $\left.\psi_{2}\right|_{1+\pi_{i} \mathcal{O}_{\mathfrak{p}_{i}}}$ are trivial for all $i \neq j$;

$-\left.\psi_{2}\right|_{1+\pi_{j} \mathcal{O}_{\mathfrak{p}_{j}}}$ is a nontrivial character which factors through $\left(1+\pi_{j} \mathcal{O}_{\mathfrak{p}_{j}}\right) /(1+$ $\left.\pi_{j}^{2} \mathcal{O}_{\mathfrak{p}_{j}}\right)$

$-\left.\psi_{1}\right|_{1+\pi_{j} \mathcal{O}_{\mathfrak{p}_{j}}}=\left(\left.\psi_{2}\right|_{1+\pi_{j} \mathcal{O}_{\mathfrak{p}_{j}}}\right)^{-2} ;$ and

- the characters $\left.\psi_{1}\right|_{\Delta_{p}}$ and $\left.\psi_{2}\right|_{\Delta_{p}}$ are uniquely determined by the condition that the point $\chi_{l}$ belongs to the component $\mathcal{W}_{\omega}$ of $\mathcal{W}$.

It is straightforward to verify that $T_{i, \chi_{l}}=0$ for all $i \neq j$, and $v_{p}\left(T_{j, \chi_{l}}\right)=\frac{1}{p-1} \in(0,1)$.

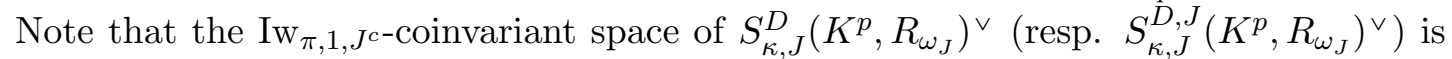
given by $S_{\kappa, J}^{D}\left(K^{p}, R_{\omega_{J}}\right)^{\vee} \otimes_{R_{\omega_{J}} \llbracket P_{J c}^{\prime} \rrbracket} R_{j}$ (resp. $\left.S_{\kappa, J}^{D, J}\left(K^{p}, R_{\omega_{J}}\right)^{\vee} \otimes_{R_{\omega_{J}} \llbracket P_{J c}^{\prime} \rrbracket} R_{j}\right)$, and this space admits an action of $\operatorname{Iw}_{\pi, J^{c}} / \mathrm{Iw}_{\pi, 1, J^{c}} \cong \Delta_{J^{c}}$.

Define a homomorphism $\tau_{l}: R_{j} \rightarrow \mathbb{C}_{p}$ of $\mathbb{Z}_{p}$-algebras with $\tau_{l}\left(T_{j}\right)=T_{j, \chi_{l}}$ and we use $M_{\tau_{l}} \in \mathrm{M}_{\infty}\left(\mathbb{C}_{p}\right)$ to denote the infinite matrix obtained by applying $\tau_{l}$ to entries of $M_{R_{j}}$.

Now we consider another character $\omega_{J}^{\prime}=\left(\omega_{1}^{-1}, \omega_{2}\right): H_{J} \rightarrow \mathbb{Z}_{p}^{\times}$, and obtain another character $\omega^{\prime}=\left(\omega_{J}^{\prime}, \omega_{J^{c}}\right): H \rightarrow \mathbb{Z}_{p}^{\times}$. We construct another point $\chi_{l}^{\prime} \in \mathcal{W}\left(\mathbb{C}_{p}\right)$ whose associated character $\kappa_{l}^{\prime}: \mathcal{O}_{p}^{\times} \times \mathcal{O}_{p}^{\times} \rightarrow \mathbb{C}_{p}^{\times}$corresponds to the triple $\left(n, \nu, \psi^{\prime}=\left(\psi_{1}^{-1}, \psi_{2}\right)\right)$. It belongs to the weight disc $\mathcal{W}_{\omega^{\prime}}$. Applying the homomorphisms $R \llbracket P_{J^{c}}^{\prime} \rrbracket \rightarrow R_{\omega_{J}^{\prime}} \llbracket P_{J^{c}}^{\prime} \rrbracket \rightarrow R_{\omega_{J}^{\prime}} \rightarrow R_{j}$, we get matrices $M_{R_{\omega_{J}^{\prime}}^{\prime} \llbracket P_{J^{c}}^{\prime} \rrbracket}, M_{R_{\omega_{J}^{\prime}}}^{\prime}$ and $M_{R_{j}}^{\prime}$ as before. Applying the homomorphism $\tau_{l}$ to entries of $M_{R_{j}}^{\prime}$, we get a matrix $M_{\tau_{l}}^{\prime} \in \mathrm{M}_{\infty}\left(\mathbb{C}_{p}\right)$.

Define $t \in \mathbb{N}^{I}$ by $t_{j}=2$ and $t_{i}=1$ for all $i \neq j$. Since we have $\left|\Delta_{J^{c}}\right|$ choices of the characters $\omega_{J^{c}}: \Delta_{J^{c}} \rightarrow \mathbb{Z}_{p}^{\times}$, and $\operatorname{dim} S_{k, w}^{D}\left(K^{p} \operatorname{Iw}_{\pi^{t}}, \psi\right)=s p(l+1)$, it follows from Proposition 3.8.5 that there are $s p(l+1)\left|\Delta_{J^{c}}\right|=S p(l+1)$ pairs of the slopes of the Newton polygons of $M_{\tau_{l}}$ and $M_{\tau_{l}}^{\prime}$, such that the slopes in each pair sum to $l+1$. Hence the total sum of these slopes is $S p(l+1)^{2}$. Since the matrix $M_{R_{j}} \in \mathrm{M}_{\infty}\left(R_{j}\right)$ is $\underline{\lambda}$-Hodge bounded, it follows from the construction of the matrix $M_{\tau_{l}}$ that the sum of the first $S p(l+1)$ slopes of $M_{\tau_{l}}$ is at least $v_{p}\left(T_{j, \chi_{l}}\right) \sum_{k=0}^{S p(l+1)-1} \lambda_{k}$. For the same reason, the first $S p(l+1)$ $S p(l+1)-1$

slopes of $M_{\tau_{l}^{\prime}}$ is at least $v_{p}\left(T_{j, \chi_{l}^{\prime}}\right) \sum_{k=0}^{S p(l+1)-1} \lambda_{k}$. As $v_{p}\left(T_{j, \chi_{l}}\right)=v_{p}\left(T_{j, \chi_{l}^{\prime}}\right)=\frac{1}{p-1}$, we have $S p(l+1)^{2}=v_{p}\left(T_{j, \chi_{l}}\right) \sum_{k=0}^{S p(l+1)-1} \lambda_{k}+v_{p}\left(T_{j, \chi_{l}^{\prime}}\right) \sum_{k=0}^{S p(l+1)-1} \lambda_{k}$. Therefore the first $S p(l+1)$ slopes of the matrix $M_{\tau_{l}}$ (resp. $\left.M_{\tau_{l}^{\prime}}\right)$ sum to exactly $v_{p}\left(T_{j, \chi_{l}}\right) \sum_{k=0}^{S p(l+1)-1} \lambda_{k}=\frac{1}{2} S p(l+1)^{2}$ (resp. $\left.v_{p}\left(T_{j, \chi_{l}^{\prime}}\right) \sum_{k=0}^{S p(l+1)-1} \lambda_{k}=\frac{1}{2} S p(l+1)^{2}\right)$. Let

$$
\operatorname{char}\left(M_{R_{j}}\right):=\sum_{n \geqslant 0} c_{n} X^{n} \in \mathbb{Z}_{p} \llbracket T_{j} \rrbracket \llbracket X \rrbracket \subset R_{j} \llbracket X \rrbracket
$$

be the characteristic power series of $M_{R_{j}}$, with $c_{n} \in \mathbb{Z}_{p} \llbracket T_{j} \rrbracket$. The above discussion implies that the Newton polygon of $\sum_{n \geqslant 0} c_{n}\left(T_{j, \chi_{l}}\right) X^{n}$ passes through $\left(S p(l+1), \mu_{S p(l+1)} v_{p}\left(T_{j, \chi_{l}}\right)\right)$ for all $l \in 2 \mathbb{Z}_{\geqslant 0}$. For simplicity, we denote $n_{l+1}=S p(l+1)$ for $l \in 2 \mathbb{Z}_{\geqslant 0}$.

Now we can run the exact argument in the proof of [20, Theorem 1.3]. We give a summary of their results which will be used in our argument: 
(1) Write $c_{n}\left(T_{j}\right)=\sum_{m \geqslant 0} b_{n, m} T_{j}^{m}$, with $b_{n, m} \in \mathbb{Z}_{p}$. Then $v_{p}\left(b_{n, m}\right) \geqslant \max \left\{\mu_{n}-m, 0\right\}$. In particular, for $t_{j} \in \mathbb{C}_{p}$ with $v_{p}\left(t_{j}\right) \in(0,1)$, we have $v_{p}\left(c_{n}\left(t_{j}\right)\right) \geqslant \mu_{n} v_{p}\left(t_{j}\right)$ for all $n \in \mathbb{Z}_{\geqslant 0}$. The equality holds if and only if $b_{n, \mu_{n}} \in \mathbb{Z}_{p}^{\times}$.

(2) For any $l \in 2 \mathbb{Z}_{\geqslant 0}$, there exist two integers $n_{l+1}^{-} \in\left[n_{l+1}-S, n_{l+1}\right]$ and $n_{l+1}^{+} \in$ $\left[n_{l+1}, n_{l+1}+S\right]$, such that for any $t_{j} \in \mathbb{C}_{p}$ with $v_{p}\left(t_{j}\right) \in(0,1)$, we have the following.

- If $n_{l+1}^{-} \neq n_{l+1}^{+}$, then the points $\left(n_{l+1}^{-}, \mu_{n_{l+1}^{-}} v_{p}\left(t_{j}\right)\right)$ and $\left(n_{l+1}^{+}, \mu_{n_{l+1}^{+}} v_{p}\left(t_{j}\right)\right)$ are two consecutive vertices of the Newton polygon of $\sum_{n \geqslant 0} c_{n}\left(t_{j}\right) X^{n}$. Moreover, the line segment connecting these two vertices has slope $(l+1)(p-1) v_{p}\left(t_{j}\right)$;

- If $n_{l+1}^{-}=n_{l+1}=n_{l+1}^{+}$, then $\left(n_{l+1}, \mu_{n_{l+1} v_{p}\left(t_{j}\right)}\right)$ is a vertex of the Newton polygon of $\sum_{n \geqslant 0} c_{n}\left(t_{j}\right) X^{n}$.

Now we consider the matrix $\bar{M} \in \mathrm{M}_{\infty}\left(\mathbb{F}_{p} \llbracket T_{j} \rrbracket\right)$. Its characteristic power series $\operatorname{char}(\bar{M})=$ $\sum_{n \geqslant 0} d_{n}\left(T_{j}\right) X^{n}$ can be obtained by applying the homomorphism $R_{j} \rightarrow \mathbb{F}_{p} \llbracket T_{j} \rrbracket$ to the coefficients of the power series $\operatorname{char}\left(M_{R_{j}}\right)=\sum_{n \geqslant 0} c_{n}\left(T_{j}\right) X^{n}$. We define the lower bound polygon of $\operatorname{char}(\bar{M})$ to be the lower convex hull of the points $\left(n, \mu_{n}\right)$, for all $n \in \mathbb{Z}_{\geqslant 0}$. Since $\bar{M}$ is $\underline{\lambda}$-Hodge bounded, the Newton polygon of $\operatorname{char}(\bar{M})$ always lies on or above its lower bound polygon. We observe the following two facts:

- The kernel of the composite $\mathbb{Z}_{p} \llbracket T_{j} \rrbracket \rightarrow R_{j} \rightarrow \mathbb{F}_{p} \llbracket T_{j} \rrbracket$ is the principal ideal generated by $p$.

- The $x$-coordinates of the line segment of the lower bound polygon with slope $(l+$ 1) $(p-1)$ belong to the interval $\left[n_{l+1}-S, n_{l+1}+S\right]$.

Combined the results from 20], these facts imply the following.

If $n_{l+1}^{-} \neq n_{l+1}^{+}$, the points $\left(n_{l+1}^{-}, v_{T_{j}}\left(d_{n_{l+1}^{-}}\left(T_{j}\right)\right)\right)$ and $\left(n_{l+1}^{+}, v_{T_{i}}\left(d_{n_{l+1}^{+}}\left(T_{j}\right)\right)\right)$ lie on the lower bound polygon and the slope of the line segment connecting these two vertices has slope $\lambda_{n_{l+1}}=(l+1)(p-1)$; if $n_{l+1}^{-}=n_{l+1}=n_{l+1}^{+},\left(n_{l+1}, v_{T_{j}}\left(d_{n_{l+1}}\left(T_{j}\right)\right)\right)$ lies on the lower bound polygon. In both cases, the slopes of the Newton polygon of $\operatorname{char}(\bar{M})$ on the left of the point $\left(n_{l+1}^{-}, v_{T_{j}}\left(d_{n_{l+1}^{-}}\left(T_{j}\right)\right)\right)$ belong to $\left[0, \lambda_{n_{l+1}}\right)$, and the slopes of the Newton polygon of $\operatorname{char}(\bar{M})$ on the right of the point $\left(n_{l+1}^{+}, v_{T_{j}}\left(d_{n_{l+1}^{+}}\left(T_{j}\right)\right)\right)$ belong to $\left(\lambda_{n_{l+1}}, \infty\right)$. In particular, $\left(n_{l+1}^{-}, v_{T_{j}}\left(d_{n_{l+1}^{-}}\left(T_{j}\right)\right)\right)$ and $\left(n_{l+1}^{+}, v_{T_{j}}\left(d_{n_{l+1}^{+}}\left(T_{j}\right)\right)\right)$ are touching vertices of the Newton polygon of $\operatorname{char}(\bar{M})=\sum_{n \geqslant 0} d_{n}\left(T_{j}\right) X^{n}$.

Notice that $(0,0)$ is always a touching vertex of $\bar{M}$. The above construction of the numbers $n_{l+1}^{+}$'s also applies to the point $(0,0)$. It follows that we can find an integer $n_{0}^{+} \in[0, S]$, such that $\left(n_{0}^{+}, v_{T_{j}}\left(d_{n_{0}^{+}}\left(T_{j}\right)\right)\right)$ is a touching vertex of the Newton polygon of $\operatorname{char}(\bar{M})$, and the slopes of this Newton polygon on the left of the point $\left(n_{0}^{+}, v_{T_{j}}\left(d_{n_{0}^{+}}\left(T_{j}\right)\right)\right)$ are all 0 . We denote $n_{0}^{-}=0$ for simplicity.

Let $\Omega_{\omega_{J}}:=\left\{n_{0}^{-}, n_{0}^{+}\right\} \cup\left\{n_{l}^{-}, n_{l}^{+} \mid l \in 1+2 \mathbb{Z}_{\geqslant 0}\right\}=\left\{n_{0}^{-} \leqslant n_{0}^{+} \leqslant n_{1}^{-} \leqslant n_{1}^{+} \leqslant n_{3}^{-} \leqslant \ldots\right\}$. We can apply Theorem 2.3 .13 to the matrix $M \in \mathrm{M}_{\infty}\left(R_{\omega_{J}} \llbracket P_{J^{c}}^{\prime} \rrbracket\right)$ and the set $\Omega_{\omega_{J}}$, and get the following.

Proposition 4.6.1. For every character $\omega_{J}: H_{J} \rightarrow \mathbb{Z}_{p}^{\times}$, there exist a basis $\left\{v_{m} \mid m \in \mathbb{N}\right\}$ and a filtration $\left\{\tilde{V}_{\alpha} \mid \alpha \in \Omega_{\omega_{J}}\right\}$ of the left $R_{\omega_{J}} \llbracket P_{J}^{\prime} \rrbracket$-module $S_{\kappa, J}^{D, J}\left(K^{p}, R_{\omega_{J}}\right)^{\vee}$ with the following properties. 
(1) If we use $N \in \mathrm{M}_{\infty}\left(R_{\omega_{J}} \llbracket P_{J^{c}}^{\prime} \rrbracket\right)$ to denote the matrix corresponding to the $U_{\pi_{j} \text {-operator }}$ on $S_{\kappa, J}^{D, J}\left(K^{p}, R_{\omega_{J}}\right)^{\vee}$ under the basis $\left\{v_{m} \mid m \in \mathbb{N}\right\}$, the matrix $N$ is $\underline{\lambda}$-Hodge bounded with respect to the element $T_{j} \in R_{\omega_{J}}$.

(2) The set $\left\{\tilde{V}_{\alpha} \mid \alpha \in \Omega_{\omega_{J}}\right\}$ is an increasing filtration of $S_{\kappa, J}^{D, J}\left(K^{p}, R_{\omega_{J}}\right)^{\vee}$; more precisely, we have $(0)=\tilde{V}_{n_{0}^{-}} \subset \tilde{V}_{n_{0}^{+}} \subset \tilde{V}_{n_{1}^{-}} \subset \tilde{V}_{n_{1}^{+}} \subset \ldots$

(3) For every $\alpha \in \Omega_{\omega_{J}}, \tilde{V}_{\alpha}$ is a free $R_{\omega_{J}} \llbracket P_{J^{c}}^{\prime} \rrbracket$-submodule of $S_{\kappa, J}^{D, J}\left(K^{p}, R_{\omega_{J}}\right)^{\vee}$ of finite rank $d_{\alpha} \geqslant 0$, and $\left\{v_{m} \mid m=1, \ldots, d_{\alpha}\right\}$ is a basis of $\tilde{V}_{\alpha}$.

(4) For every $\alpha \in \Omega_{\omega_{J}}, \tilde{V}_{\alpha}$ is stable under the $U_{\pi_{j}}$-operator. We use $R_{\alpha} \in \mathrm{M}_{d_{\alpha}}\left(R_{\omega_{J}} \llbracket P_{J^{c}}^{\prime} \rrbracket\right)$ to denote the matrix corresponding to the $U_{\pi_{j}}$-operator on $\tilde{V}_{\alpha}$ under the basis $\left\{v_{m} \mid m=\right.$ $\left.1, \ldots, d_{\alpha}\right\}$. In particular, the matrix $N$ can be written in the blockwise form:

$$
N=d_{\alpha}\left[\begin{array}{c:c}
d_{\alpha} & \vdots \\
A_{\alpha} & B_{\alpha} \\
\hdashline 0 & D_{\alpha}
\end{array}\right] \in \mathrm{M}_{\infty}\left(R_{\omega_{J}} \llbracket P_{J^{c}}^{\prime} \rrbracket\right)
$$

(5) Let $\alpha \leqslant \beta$ be two consecutive elements in $\Omega_{\omega_{J}}$. The matrix $A_{\beta}$ can be written $d_{\alpha}$

in the block uppertriangular forms: $d_{\alpha}\left[\begin{array}{c:c}A_{11} & A_{12} \\ \hdashline 0 & A_{22}\end{array}\right]$ with $A_{11}=A_{\alpha}$. We use $\bar{A}_{22} \in \mathrm{M}_{d}\left(\mathbb{F}_{p} \llbracket T_{j} \rrbracket\right)$ to denote the matrix obtained by applying the homomorphism $R_{\omega_{J}} \llbracket P_{J^{c}}^{\prime} \rrbracket \rightarrow \mathbb{F}_{p} \llbracket T_{j} \rrbracket$ to entries of $A_{22}$, where $d=d_{\beta}-d_{\alpha}$. Then the slopes of the Newton polygon of $\bar{A}_{22}$ (with respect to the $T_{j}$-adic valuation) belong to $\left\{\lambda_{l}\right\}$ if $\alpha=n_{l}^{-}, \beta=n_{l}^{+}$, and belong to $\left(\lambda_{l}, \lambda_{l^{\prime}}\right)$ if $\alpha=n_{l}^{+}, \beta=n_{l^{\prime}}^{-}$.

\section{Proof of the MaIN THEOREM}

Notation 5.0.1. In this section, we write $I=\left\{j_{1}, j_{2}, \ldots, j_{g}\right\}$. For $1 \leqslant l \leqslant g$, we denote by $J_{l}$ the subset $\left\{j_{1}, \ldots, j_{l}\right\}$ of $I$. We will drop the letter $J$ or $j$ in the previous notations for simplicity. More precisely, for $1 \leqslant l \leqslant g$, we make the following.

(1) Let $\mathfrak{p}_{l}$ be the prime of $F$ corresponding to the embedding $j_{l} \in I$ and $\pi_{l}=\pi_{j_{l}}$ be the uniformizer of $\mathcal{O}_{\mathfrak{p}_{l}}$ we fixed before.

(2) Let $H_{l}:=H_{J_{l}}$ denote the torsion subgroup of $\mathcal{O}_{p, J}^{\times} \times \mathbb{Z}_{p}^{\times}$and $\delta_{l}$ be the torsion subgroup of $\mathcal{O}_{\mathfrak{p}_{l}}^{\times}$.

(3) Let $\Lambda_{l}$ and $R_{l}$ denote the ring $\Lambda_{J_{l}}=\mathbb{Z}_{p} \llbracket \mathcal{O}_{p, J}^{\times} \times \mathbb{Z}_{p}^{\times} \rrbracket$ and $\Lambda_{J_{l}}^{>1 / p}$, respectively.

(4) Let $T_{l} \in \Lambda_{l}$ be the element $T_{j_{l}}$ defined in \$3.1. In particular, we can write $\Lambda_{l}=$ $\mathbb{Z}_{p}\left[H_{l}\right] \otimes_{\mathbb{Z}_{p}} \mathbb{Z}_{p} \llbracket\left(T_{m}\right)_{1 \leqslant m \leqslant l}, T \rrbracket$ and $R_{l}=\mathbb{Z}_{p}\left[H_{l}\right] \otimes_{\mathbb{Z}_{p}} \mathbb{Z}_{p} \llbracket\left(T_{m}, \frac{p}{T_{m}}\right)_{1 \leqslant m \leqslant l}, T \rrbracket$.

5.1. Properties of the filtration on the space of integral $p$-adic automorphic forms. We apply the construction in 84.6 to the set $J_{1}$ consisting of a single element $j_{1}$. To be more precise, we fix a character $\omega_{1}=\left(\eta_{1}, \eta\right): H_{1}=\Delta_{1} \times \Delta \rightarrow \mathbb{Z}_{p}^{\times}$. Let $R_{1, \omega_{1}}=R_{1} \otimes_{\mathbb{Z}_{p}\left[H_{1}\right], \omega_{1}} \mathbb{Z}_{p}$, which is isomorphic to $\mathbb{Z}_{p} \llbracket T_{1}, \frac{p}{T_{1}}, T \rrbracket$ and $R_{2, \omega_{2}}=R_{2} \otimes_{\mathbb{Z}_{p}\left[H_{1}\right], \omega_{1}} \mathbb{Z}_{p}$.

We apply Proposition 4.6 .1 to the space $S_{\kappa, J_{1}}^{D, J_{1}}\left(K^{p}, R_{1, \omega_{1}}\right)^{\vee}$ and the $U_{\pi_{1}}$-operator on it. Then we get a set $\Omega_{\omega_{1}}=\left\{n_{l}^{-}, n_{l}^{+} \mid l \in\{0\} \cup 1+2 \mathbb{Z}_{\geqslant 0}\right\}$, a basis $\left\{v_{m} \mid m \in \mathbb{N}\right\}$ and a filtration $\left\{\tilde{V}_{\alpha} \mid \alpha \in \Omega_{\omega_{1}}\right\}$ of the space $S_{\kappa, J_{1}}^{D, J_{1}}\left(K^{p}, R_{1, \omega_{1}}\right)^{\vee}$. By Remark 3.7.7, we have a surjective map $S_{\kappa, J_{1}}^{D, J_{1}}\left(K^{p}, R_{2, \omega_{1}}\right)^{\vee} \rightarrow S_{\kappa, J_{2}}^{D, J_{1}}\left(K^{p}, R_{2, \omega_{1}}\right)^{\vee}$, which identifies the latter space as the $B\left(\mathcal{O}_{\mathfrak{p}_{2}}\right)$ coinvariants of the first space. For $\alpha \in \Omega_{\omega_{1}}$, we use $V_{\alpha}$ to denote the image of $\tilde{V}_{\alpha} \otimes_{R_{1, \omega_{1}}} R_{2, \omega_{2}}$ under this map. 
Proposition 5.1.1. For all $\alpha \in \Omega_{\omega_{1}}$, the space $V_{\alpha}$ is stable under the $U_{\pi_{2}}$-operator on $S_{\kappa, J_{2}}^{D, J_{1}}\left(K^{p}, R_{2, \omega_{1}}\right)^{\vee}$.

Proof. Throughout the proof, we use $R_{1}^{\prime}$ and $R_{2}^{\prime}$ to denote the rings $R_{1, \omega_{1}}$ and $R_{2, \omega_{2}}$, respectively.



We apply the homomorphisms $R_{1}^{\prime} \llbracket P_{J_{1}^{c}}^{\prime} \rrbracket \rightarrow R_{1}^{\prime} \rightarrow \mathbb{F}_{p} \llbracket T_{1} \rrbracket$ to the matrix $A_{\alpha} \in \mathrm{M}_{d_{\alpha}}\left(R_{1}^{\prime} \llbracket P_{J_{1}^{c}}^{\prime} \rrbracket\right)$, and obtain matrices $A_{\alpha, R_{1}^{\prime}} \mathrm{M}_{d_{\alpha}}\left(R_{1}^{\prime}\right)$ and $\bar{A}_{\alpha} \in \mathrm{M}_{d_{\alpha}}\left(\mathbb{F}_{p} \llbracket T_{1} \rrbracket\right)$. We use $f(X)=\operatorname{det}\left(X \cdot I_{d_{\alpha}}-\right.$ $\left.A_{\alpha, R_{1}^{\prime}}\right)=X^{d_{\alpha}}+a_{d_{\alpha}-1} X^{d_{\alpha}-1}+\cdot+a_{0} \in R_{1}^{\prime}[X]$ to denote the characteristic polynomial of $A_{\alpha, R_{1}^{\prime}}$. Since the matrix $\bar{A}_{\alpha}$ is strictly $\underline{\lambda}^{\left[d_{\alpha}\right]}$-Hodge bounded, we can write $a_{0}=T_{1}^{n_{\alpha}} \cdot a_{0}^{\prime}$, where $n_{\alpha}=\sum_{k=0}^{d_{\alpha}-1} \lambda_{k}$ and $a_{0}^{\prime}$ is a unit in $R_{1}^{\prime}$.

As $N$ is $\underline{\lambda}$-Hodge bounded and $\lim _{k \rightarrow \infty} \lambda_{k}=\infty$, we can find an element $\beta \geqslant \alpha$ in $\Omega_{\omega_{1}}$ with the following property: if we write the matrix $N$ in the block uppertriangular form $N=d_{\beta}\left[\begin{array}{c:c}A_{\beta} & B_{\beta} \\ \hdashline 0 & D_{\beta}\end{array}\right]$, then $D_{\beta} \in \mathrm{M}_{\infty}\left(T_{1}^{n_{\alpha}+1} R_{1}^{\prime} \llbracket P_{J_{1}^{c}}^{\prime} \rrbracket\right)$. We write $f\left(D_{\beta}\right)=a_{0} I_{\infty}+a_{1} D_{\beta}+$ $\cdots+D_{\beta}^{d_{\alpha}}=T_{1}^{n_{\alpha}}\left(a_{0}^{\prime} I_{\infty}+\left(T_{1}^{-n_{\alpha}} D_{\beta}\right)\left(a_{1}+\cdots+D_{\beta}^{d_{\alpha}-1}\right)\right)$. Since $T_{1}^{-n_{\alpha}} D_{\beta} \in \mathrm{M}_{\infty}\left(T_{1} R_{1}^{\prime} \llbracket P_{J_{1}^{c}}^{\prime} \rrbracket\right)$ and $a_{0}^{\prime}$ is a unit in $R_{1}^{\prime}$, the matrix $D_{\beta}^{\prime}=a_{0}^{\prime} I_{\infty}+\left(T_{1}^{-n_{\alpha}} D_{\beta}\right)\left(a_{1}+\cdots+D_{\beta}^{d_{\alpha}-1}\right)$ belongs to $\mathrm{GL}_{\infty}\left(R_{1}^{\prime} \llbracket P_{J_{1}^{c}}^{\prime} \rrbracket\right)$ by Lemma 2.3 .9 .

By Proposition 4.6.1, the matrix $A_{\beta} \in \mathrm{M}_{d_{\beta}}\left(R_{1}^{\prime} \llbracket P_{J_{1}^{c}}^{\prime} \rrbracket\right)$ is of the block uppertriangular $d_{\alpha}$ form $d_{\alpha}\left[\begin{array}{c:c}A_{11} & A_{12} \\ \hdashline 0 & A_{22}\end{array}\right]$ with $A_{11}=A_{\alpha}$.

Denote $d=d_{\beta}-d_{\alpha}$ and $g(X)=\operatorname{det}\left(X \cdot I_{d}-A_{22, R_{1}^{\prime}}\right) \in R_{1}^{\prime}[X]$ for the characteristic polynomial of $A_{22, R_{1}^{\prime}}$. It follows from Proposition 4.6.1 that the slopes of the Newton polygon of $\bar{A}_{11} \in \mathrm{M}_{d_{\alpha}}\left(\mathbb{F}_{p} \llbracket T_{1} \rrbracket\right)$ are strictly less than the slopes of the Newton polygon of $\bar{A}_{22} \in \mathrm{M}_{d^{\prime}}\left(\mathbb{F}_{p} \llbracket T_{1} \rrbracket\right)$ (under the $T_{1}$-adic valuation). We use $\bar{f}(X)$ (resp. $\bar{g}(X)$ ) to denote the image of $f(X)$ (resp. $g(X))$ under the homomorphism $R_{1}^{\prime} \rightarrow \mathbb{F}_{p} \llbracket T_{1} \rrbracket$. Then $\bar{f}(X)$ and $\bar{g}(X)$ are coprime in $\mathbb{F}_{p}\left(\left(T_{1}\right)\right)[X]$. By $[22$, Chapter $1 \S 4], f$ and $g$ are strictly coprime in $R_{1}^{\prime}\left[\frac{1}{T_{1}}\right][X]$. So we can find $u(X), v(X) \in R_{1}^{\prime}[X]$ and $m_{\alpha} \in \mathbb{Z}_{\geqslant 0}$, such that

$$
f(X) u(X)+g(X) v(X)=T_{1}^{m_{\alpha}}
$$

in $R_{1}^{\prime}[X]$. By Hamilton-Cayley's Theorem (over the commutative ring $R_{1}^{\prime}$ ), we have $f\left(A_{11, R_{1}^{\prime}}\right)=$ 0 and $g\left(A_{22, R_{1}^{\prime}}\right)=0$. Hence we have $f\left(A_{22, R_{1}^{\prime}}\right) u\left(A_{22, R_{1}^{\prime}}\right)=T_{1}^{m_{\alpha}} I_{d}$ in $\mathrm{M}_{d}\left(R_{1}^{\prime}\right)$.

We use $\mathcal{I}_{1} \subset R_{1}^{\prime} \llbracket P_{J_{1}^{c}}^{\prime} \rrbracket$ and $\mathcal{I}_{2} \subset R_{2}^{\prime} \llbracket P_{J_{1}^{c}}^{\prime} \rrbracket$ to denote the augmentation ideal of the corresponding complete group algebras, respectively. In summary of the above discussion, 
the matrix $N \in \mathrm{M}_{\infty}\left(R_{1}^{\prime} \llbracket P_{J_{1}^{c}}^{\prime} \rrbracket\right)$ can be written in the following block uppertriangular form:

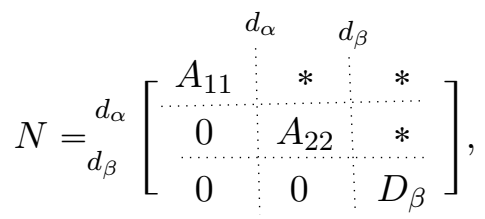

such that the matrix $f(N)=\left[\begin{array}{ccc}f\left(A_{11}\right) & * & * \\ 0 & f\left(A_{22}\right) & * \\ 0 & 0 & f\left(D_{\beta}\right)\end{array}\right]$ satisfies the following properties:

- $f\left(A_{11}\right) \in \mathrm{M}_{d_{\alpha}}\left(\mathcal{I}_{1}\right)$,

- $f\left(A_{22}\right) u\left(A_{22}\right)-T_{1}^{m_{\alpha}} \in \mathrm{M}_{d}\left(\mathcal{I}_{1}\right)$,

- $f\left(D_{\beta}\right)=T_{1}^{n_{\alpha}} D_{\beta}^{\prime}$, for some $D_{\beta}^{\prime} \in \mathrm{GL}_{\infty}\left(R_{1}^{\prime} \llbracket P_{J_{1}^{c}}^{\prime} \rrbracket\right)$.

Under the basis $\left\{v_{m} \mid m \in \mathbb{N}\right\}$, we have an $R_{1}^{\prime} \llbracket P_{J_{1}^{c}}^{\prime} \rrbracket$-linear isomorphism $S_{\kappa_{1}, J_{1}}^{D, J_{1}}\left(K^{p}, R_{1}^{\prime}\right)^{\vee} \cong$ $\prod_{m=1}^{\infty} R_{1}^{\prime} \llbracket P_{J_{1}^{c}}^{\prime} \rrbracket$.

For any positive integer $k$, we denote by $\mathfrak{R}_{k}$ the quotient $R_{1}^{\prime} \llbracket P_{J_{1}^{c}}^{\prime} \rrbracket / \mathcal{I}_{1}^{k}$. The above isomorphism induces an isomorphism

$$
S_{\kappa_{1}, J_{1}}^{D, J_{1}}\left(K^{p}, R_{1}^{\prime}\right)^{\vee} \otimes_{R_{1}^{\prime} \llbracket P_{J_{1}^{\prime}}^{\prime} \rrbracket} \mathfrak{R}_{k} \cong \prod_{m=1}^{\infty} \mathfrak{R}_{k}
$$

Since the $U_{\pi_{1}}$-operator on $S_{\kappa_{1}, J_{1}}^{D, J_{1}}\left(K^{p}, R_{1}^{\prime}\right)^{\vee}$ is $R_{1}^{\prime} \llbracket P_{J_{1}^{c}}^{\prime} \rrbracket$-linear, it induces an $\mathfrak{R}_{k^{-}}$-linear map on $S_{\kappa_{1}, J_{1}}^{D, J_{1}}\left(K^{p}, R_{1}^{\prime}\right)^{\vee} \otimes_{R_{1}^{\prime} \llbracket P_{J_{1}^{c}}^{\prime} \rrbracket} \mathfrak{R}_{k}$. Under the above isomorphism, this operator corresponds to the infinite matrix $N_{\mathfrak{R}_{k}}$, and hence $f\left(U_{\pi_{1}}\right)$ corresponds to the matrix $f(N)_{\mathfrak{R}_{k}}$. From the above discussion, we have the following facts for $f(N)_{\mathfrak{R}_{k}}$.

- The submatrix $f\left(A_{11}\right)_{\mathfrak{R}_{k}}$ is nilpotent as $f\left(A_{11}\right) \in \mathrm{M}_{d_{\alpha}}\left(\mathcal{I}_{1}\right)$; more precisely, we have $f\left(A_{11}\right)_{\mathfrak{R}_{k}}^{k}=0$.

- There exists a matrix $B_{k} \in \mathrm{M}_{d}\left(\Re_{k}\right)$, such that $f\left(A_{22}\right)_{\mathfrak{R}_{k}} \cdot B_{k}=B_{k} \cdot f\left(A_{22}\right)_{\mathfrak{R}_{k}}=$ $T_{1}^{m_{\alpha} k} \cdot I_{d}$; in fact, if we write $f\left(A_{22}\right) u\left(A_{22}\right)=T_{1}^{m_{\alpha}} \cdot I_{d}+E$, with $E \in \mathrm{M}_{d}\left(\mathcal{I}_{1}\right)$, we can choose the matrix $B_{k}$ to be $u\left(A_{22}\right)\left(\sum_{l=0}^{k-1} T_{1}^{m_{\alpha} l} E^{k-1-l}\right)$ (viewed as a matrix in $\mathrm{M}_{d}\left(\mathfrak{R}_{k}\right)$ in the obvious way).

- $f\left(D_{\beta}\right)_{\Re_{k}}=T_{1}^{n_{\alpha}} D_{\beta, \Re_{k}}^{\prime}$ with $D_{\beta, \Re_{k}}^{\prime} \in \mathrm{GL}_{\infty}\left(\mathfrak{R}_{k}\right)$.

So we have

$$
\tilde{V}_{\alpha} / \mathcal{I}_{1}^{k}=\underset{m}{\lim } \operatorname{ker}\left(f\left(U_{\pi_{1}}\right)^{m}: S_{\kappa, J_{1}}^{D, J_{1}}\left(K^{p}, R_{1}^{\prime}\right)^{\vee} \otimes_{R_{1}^{\prime} \llbracket P_{J_{1}^{c}}^{\prime} \rrbracket} \Re_{k} \rightarrow S_{\kappa, J_{1}}^{D, J_{1}}\left(K^{p}, R_{1}^{\prime}\right)^{\vee} \otimes_{R_{1}^{\prime} \llbracket P_{J_{1}^{c}}^{\prime} \rrbracket} \Re_{k}\right) .
$$

Since we have a surjection $S_{\kappa, J_{1}}^{D, J_{1}}\left(K^{p}, R_{2}^{\prime}\right)^{\vee} \rightarrow S_{\kappa, J_{2}}^{D, J_{1}}\left(K^{p}, R_{2}^{\prime}\right)^{\vee}$, and $T_{1}$ is not a zero divisor in $R_{2}^{\prime} \llbracket P_{J_{1}^{c}}^{\prime} \rrbracket / \mathcal{I}_{2}^{k}$, for all $k>0$, we have

$$
V_{\alpha} / \mathcal{I}_{2}^{k}=\underset{m}{\lim } \operatorname{ker}\left(f\left(U_{\pi_{1}}\right)^{m}: S_{\kappa, J_{2}}^{D, J_{1}}\left(K^{p}, R_{2}^{\prime}\right)^{\vee} / \mathcal{I}_{2}^{k} \rightarrow S_{\kappa, J_{2}}^{D, J_{1}}\left(K^{p}, R_{2}^{\prime}\right)^{\vee} / \mathcal{I}_{2}^{k}\right.
$$

Since the operator $U_{\pi_{2}}$ commutes with $U_{\pi_{1}}$, it also commutes with $f\left(U_{\pi_{1}}\right)$. So it stabilizes $V_{\alpha} / \mathcal{I}_{2}^{k}$ for all $k \geqslant 0$. Since $V_{\alpha}=\lim _{k} V_{\alpha} / \mathcal{I}_{2}^{k}$, we see that $U_{\pi_{2}}$ stabilizes $V_{\alpha}$. 
Remark 5.1.2. The proof of Proposition 5.1.1 is a little lengthy and technical. So it would be helpful to explain the intuition of our argument. We fix a continuous homomorphism $\chi: R_{2, \omega_{2}} \rightarrow \mathbb{C}_{p}$. We will show in $\S 5.3$ below that $V_{\alpha} \otimes_{R_{2, \omega_{2}}, \chi} \mathbb{C}_{p}$ is the subspace of $S_{\kappa, J_{2}}^{D, J_{1}}\left(K^{p}, R_{2, \omega_{2}}\right)^{\vee} \otimes_{R_{2, \omega_{2}}, \chi} \mathbb{C}_{p}$ on which the slopes of the $U_{\pi_{1}}$-operator belong to the interval $\left[0, \lambda_{l} \cdot v_{p}\left(\chi\left(T_{1}\right)\right)\right)\left(\operatorname{resp} .\left[0, \lambda_{l} \cdot v_{p}\left(\chi\left(T_{1}\right)\right)\right]\right)$ if $\alpha=n_{l}^{-}$(resp. $\left.\alpha=n_{l}^{+}\right)$. Proposition 5.1.1 follows essentially from this characterization of the spaces $V_{\alpha}$ 's and the fact that the operators $U_{\pi_{1}}$ and $U_{\pi_{2}}$ commutes with each other.

5.2. A filtration on the space of $p$-adic automorphic forms with respect to all $U_{\pi_{i}}$ operators. In this section, we fix a character $\eta_{2}: \Delta_{2} \rightarrow \mathbb{Z}_{p}^{\times}$. Together with $\omega_{1}: H_{1} \rightarrow \mathbb{Z}_{p}^{\times}$, it gives a character $\omega_{2}: H_{2} \rightarrow \mathbb{Z}_{p}^{\times}$. Denote $R_{2, \omega_{2}}=R_{2} \otimes_{\mathbb{Z}_{p}\left[H_{2}\right], \omega_{2}} \mathbb{Z}_{p}$ and $V_{\alpha, \omega_{2}}=V_{\alpha} \otimes_{\mathbb{Z}_{p}\left[H_{2}\right], \omega_{2}}$ $\mathbb{Z}_{p}$ for all $\alpha \in \Omega_{\omega_{1}}$. The set $\left\{V_{\alpha, \omega_{2}} \mid \alpha \in \Omega_{\omega_{1}}\right\}$ is a filtration of $S_{\kappa, J_{2}}^{D, J_{1}}\left(K^{p}, R_{2, \omega_{2}}\right)^{\vee}$, and by Proposition 5.1.1, this filtration is stable under the $U_{\pi_{2}}$-operator. This filtration induces a filtration $\left\{V_{\alpha, \omega_{2}}^{\text {mod }} \mid \alpha \in \Omega_{\omega_{1}}\right\}$ of $S_{\kappa, J_{2}}^{D, J_{2}}\left(K^{p}, R_{2, \omega_{2}}\right)^{\vee}$. If $\gamma \leqslant \alpha$ are two consecutive elements in $\Omega_{\omega_{1}}$, we set $W_{\alpha, \Omega_{\omega_{2}}}:=V_{\alpha, \omega_{2}} / V_{\gamma, \omega_{2}}$ and $W_{\alpha, \Omega_{\omega_{2}}}^{\text {mod }}:=V_{\alpha, \omega_{2}}^{\text {mod }} / V_{\gamma, \omega_{2}}^{\text {mod }}$. Hence, $W_{\alpha, \Omega_{\omega_{2}}}$ also carries an action of the $U_{\pi_{2}}$-operator. Under the basis $\left\{v_{m} \mid m=1, \ldots, d_{\alpha}\right\}$ of $\tilde{V}_{\alpha}$, we have an isomorphism $\tilde{V}_{\alpha} \cong \prod_{k=1}^{d_{\alpha}} R_{1, \omega_{1}} \llbracket P_{J_{1}^{c}}^{\prime} \rrbracket$, which induces an isomorphism $V_{\alpha, \omega_{2}} \cong \prod_{k=1}^{d_{\alpha}} R_{2, \omega_{2}} \llbracket \mathcal{O}_{\mathfrak{p}_{2}} \times$ $P_{J_{2}^{c}}^{\prime} \rrbracket$. So we obtain an isomorphism $W_{\alpha, \omega_{2}} \cong \prod_{k=1}^{r_{\alpha}} R_{2, \omega_{2}} \llbracket \mathcal{O}_{\mathfrak{p}_{2}} \times P_{J_{2}^{c}}^{\prime} \rrbracket$, where $r_{\alpha}=d_{\alpha}-d_{\gamma}$ under the above notations. We also have isomorphisms $V_{\alpha, \omega_{2}}^{m o d} \cong \prod_{k=1}^{d_{\alpha}} R_{2, \omega_{2}} \llbracket \mathcal{O}_{\mathfrak{p}_{2}} \times P_{J_{2}^{c}}^{\prime} \rrbracket^{j_{2}-m o d}$ and $W_{\alpha, \omega_{2}}^{\text {mod }} \cong \prod_{k=1}^{r_{\alpha}} R_{2, \omega_{2}} \llbracket \mathcal{O}_{\mathfrak{p}_{2}} \times P_{J_{2}^{c}}^{\prime} \rrbracket^{j_{2}-m o d}$. Since $V_{\alpha, \omega_{2}}$ is stable under the $U_{\pi_{1}}$ and $U_{\pi_{2}}$ operators, a similar computation in $\$ 4.5$ shows that $V_{\alpha, \omega_{2}}^{\text {mod }}$ is also stable under the $U_{\pi_{1}}$ and $U_{\pi_{2}}$-operators. Hence we have well defined $U_{\pi_{1}}$ and $U_{\pi_{2}}$-operators on $W_{\alpha, \omega_{2}}^{\text {mod }}$ for all $\alpha$.

Fix $\alpha \in \Omega_{\omega_{1}}$ in the following discussion. We will construct a filtration of the graded piece $W_{\alpha, \omega_{2}}^{\text {mod }}$ adapted to the $U_{\pi_{2}}$-operator based on a similar idea we used in $\$ 4.6$.

Under the above isomorphisms, we choose a basis $\left\{f_{k, m} \mid 1 \leqslant k \leqslant r_{\alpha}, m \in \mathbb{Z}_{\geqslant 0}\right\}$ of $W_{\alpha, \omega_{2}}$ as a left $R_{2, \omega_{2}} \llbracket P_{J_{2}^{c}}^{\prime} \rrbracket$-module, such that $\left\{f_{k, m} \mid m \in \mathbb{Z}_{\geqslant 0}\right\}$ is the Mahler basis of the $k$-th direct factor of $\prod_{k=1}^{r_{\alpha}} R_{2, \omega_{2}} \llbracket \mathcal{O}_{\mathfrak{p}_{2}} \times P_{J_{2}^{c}}^{\prime} \rrbracket$ for all $1 \leqslant k \leqslant r_{\alpha}$. We choose a basis $\left\{f_{k, m}^{m o d} \mid 1 \leqslant k \leqslant r_{\alpha}, m \in\right.$ $\left.\mathbb{Z}_{\geqslant 0}\right\}$ of $W_{\alpha, \omega_{2}}^{\text {mod }}$ in a similar way.

We use $N=\left(N_{m, n}\right)_{m, n \geqslant 0}\left(\right.$ resp. $\left.M=\left(M_{m, n}\right)_{m, n \geqslant 0}\right)$ to denote the matrix in $\mathrm{M}_{\infty}\left(R_{2, \omega_{2}} \llbracket P_{J_{2}^{c}}^{\prime} \rrbracket\right)$ which corresponds to the $U_{\pi_{2}}$-operator on $W_{\alpha, \omega_{2}}$ (resp. $W_{\alpha, \omega_{2}}^{\text {mod }}$ ) under the basis we choose above. It follows from Propositions 4.2.1 and 4.3.1 that $N_{m, n} \in\left(T_{2}\right)^{\max \left\{\left\lfloor\frac{n}{r_{\alpha}}\right\rfloor-\left\lfloor\frac{m}{p r_{\alpha}}\right\rfloor, 0\right\}}$ for all $m, n \in \mathbb{Z}_{\geqslant 0}$. Notice that $M$ is the conjugation of $N$ by the diagonal matrix with diagonal entries $\underbrace{1,1, \ldots, 1}_{r_{\alpha}}, \underbrace{T_{2}, T_{2}, \ldots, T_{2}}_{r_{\alpha}}, \underbrace{T_{2}^{2}, T_{2}^{2}, \ldots, T_{2}^{2}}_{r_{\alpha}}, \ldots$ Define two sequence of integers $\underline{\lambda}, \underline{\mu}$ as $\lambda_{n}=\left\lfloor\frac{n}{r_{\alpha}}\right\rfloor-\left\lfloor\frac{n}{p r_{\alpha}}\right\rfloor, \mu_{0}=0, \mu_{n+1}-\mu_{n}=\lambda_{n}$ for all $n \in \mathbb{Z}_{\geqslant 0}$. Then the matrix $M$ is $\underline{\lambda}$-Hodge bounded with respect to $T_{j_{2}} \in R_{2, \omega_{J_{2}}} \llbracket P_{J_{2}^{c}}^{\prime} \rrbracket$.

Let $R_{2, \omega_{2}} \llbracket P_{J_{2}^{c}}^{\prime} \rrbracket \rightarrow R_{2, \omega_{2}}$ be the reduction map modulo the augmentation ideal of the group ring $R_{2, \omega_{J_{2}}} \llbracket P_{J_{2}^{c}}^{\prime} \rrbracket$. Under the isomorphism $R_{2, \omega_{2}} \cong \mathbb{Z}_{p} \llbracket T_{1}, T_{2}, \frac{p}{T_{1}}, \frac{p}{T_{2}}, T \rrbracket$, let $R_{2, \omega_{2}} \rightarrow$ $\mathbb{F}_{p} \llbracket T_{2} \rrbracket$ be the reduction map modulo the ideal generated by $T_{1}, \frac{p}{T_{1}}, \frac{p}{T_{2}}$ and $T$. Applying the above two homomorphisms to the entries of the matrix $M$, we obtain two matrices 
$M_{R_{2, \omega_{2}}} \in \mathrm{M}_{\infty}\left(R_{2, \omega_{2}}\right)$ and $\bar{M} \in \mathrm{M}_{\infty}\left(\mathbb{F}_{p} \llbracket T_{2} \rrbracket\right)$. Both of them are $\underline{\lambda}$-Hodge bounded with respect to $T_{2}$.

Fix an integer $l_{1} \in 2 \mathbb{Z}_{\geqslant 0}$ and a finite character $\varepsilon_{1}: 1+\pi_{1} \mathcal{O}_{\mathfrak{p}_{1}} \rightarrow \mathbb{C}_{p}^{\times}$with the following properties:

- $l_{1}+1>\frac{\lambda_{l}}{p-1}$, where $\alpha=n_{l}^{-}$or $n_{l}^{+}$; and

- $\varepsilon_{1}$ is nontrivial and factors through $\left(1+\pi_{1} \mathcal{O}_{\mathfrak{p}_{1}}\right) /\left(1+\pi_{1}^{2} \mathcal{O}_{\mathfrak{p}_{1}}\right)$.

For every character $\omega_{J_{2}^{c}}: \Delta_{J_{2}^{c}} \rightarrow \mathbb{Z}_{p}^{\times}$, we obtain a character $\omega=\left(\omega_{2}, \omega_{J_{2}^{c}}\right): H \rightarrow \mathbb{Z}_{p}^{\times}$.

Given the above datum, for any integer $l_{2} \in 2 \mathbb{Z}_{\geqslant 0}$ and any nontrivial character $\varepsilon_{2}$ : $1+\pi_{2} \mathcal{O}_{\mathfrak{p}_{2}} \rightarrow \mathbb{C}_{p}^{\times}$that factors through $1+\pi_{2}^{2} \mathcal{O}_{\mathfrak{p}_{2}}$, we construct a point $\chi_{2} \in \mathcal{W}\left(\mathbb{C}_{p}\right)$ such that its associated character $\kappa_{l_{2}}: \mathcal{O}_{p}^{\times} \times \mathcal{O}_{p}^{\times} \rightarrow \mathbb{C}_{p}^{\times}$is locally algebraic and corresponds to the triple $\left(n \in \mathbb{Z}_{\geqslant 0}^{I}, \nu \in \mathbb{Z}^{I}, \psi=\left(\psi_{1}, \psi_{2}\right)\right)$ defined as follows.

- Define $\nu:=\left(\nu_{i}\right)_{i \in I}$ by

$$
\nu_{i}= \begin{cases}-l_{1} / 2, & \text { for } i=j_{1} \\ -l_{2} / 2, & \text { for } i=j_{2}, \\ 0, & \text { otherwise }\end{cases}
$$

and $n:=-2 \nu \in \mathbb{Z}_{\geqslant 0}^{I}$.

- Let $\psi_{1}, \psi_{2}: \mathcal{O}_{p}^{\times} \rightarrow \mathbb{C}_{p}^{\times}$be two finite characters with the following properties: $\left.\psi_{1}\right|_{1+\pi_{i} \mathcal{O}_{\mathfrak{p}_{i}}}$ and $\left.\psi_{2}\right|_{1+\pi_{i} \mathcal{O}_{\mathfrak{p}_{i}}}$ are trivial for all $i \neq j_{1}, j_{2},\left.\psi_{2}\right|_{1+\pi_{1} \mathcal{O}_{\mathfrak{p}_{1}}}=\varepsilon_{1},\left.\psi_{2}\right|_{1+\pi_{2} \mathcal{O}_{\mathfrak{p}_{2}}}=$ $\varepsilon_{2}$, and $\left.\psi_{1}\right|_{1+\pi_{1} \mathcal{O}_{\mathfrak{p}_{1}}}=\varepsilon_{1}^{-2},\left.\psi_{1}\right|_{1+\pi_{2} \mathcal{O}_{\mathfrak{p}_{2}}}=\varepsilon_{2}^{-2}$; the characters $\left.\psi_{1}\right|_{\Delta_{p}}$ and $\left.\psi_{2}\right|_{\Delta_{p}}$ are determined by the condition that the point $\chi_{l_{2}}$ belongs to the component $\mathcal{W}_{\omega}$ of $\mathcal{W}$.

Under the above construction, we have $T_{i, \chi_{l_{2}}}=0$ for all $i \neq j_{1}, j_{2}$ and $v_{p}\left(T_{i, \chi_{l_{2}}}\right)=\frac{1}{p-1}$ for $i=j_{1}, j_{2}$.

Remark 5.2.1. The construction of the point $\chi_{2} \in \mathcal{W}\left(\mathbb{C}_{p}\right)$ depends on two even integers $l_{1}, l_{2}$ and two finite characters $\varepsilon_{1}, \varepsilon_{2}$. In the following paragraphs, we will construct a filtration of each graded piece $W_{\alpha, \omega_{2}}^{\text {mod }}$ adapted to the $U_{\pi_{2}}$-operator. Therefore the integer $l_{2}$ and the finite character $\varepsilon_{2}$ play similar roles as those of $l$ and $\psi_{2}$ appearing in the construction in $\S 4.6$. On the other hand, since we work over the ring $R_{2, \omega_{2}} \cong \mathbb{Z}_{p} \llbracket T_{1}, \frac{p}{T_{1}}, T_{2}, \frac{p}{T_{2}}, T \rrbracket$, we do have a restriction on the $T_{1}$-parameter of the locally algebraic weight $\chi_{2} \in \mathcal{W}\left(\mathbb{C}_{p}\right)$, that is, $v_{p}\left(T_{1, \chi_{2}}\right) \in(0,1)$. The additional assumption $l_{1}+1>\frac{\lambda_{l}}{p-1}$ is to guarantee that the space

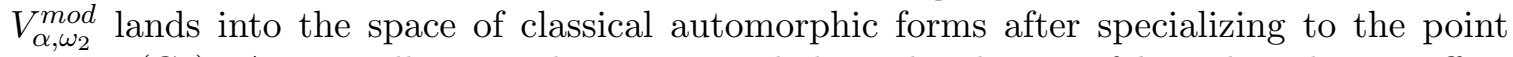
$\chi_{2} \in \mathcal{W}\left(\mathbb{C}_{p}\right)$. As we will see in the argument below, the choices of $l_{1}$ and $\varepsilon_{1}$ do not affect the filtrations adapted to the $U_{\pi_{2}}$-operator as long as they satisfy the above assumptions.

The point $\chi_{l_{2}} \in \mathcal{W}\left(\mathbb{C}_{p}\right)$ defines a homomorphism $\tau_{l_{2}}: R_{2, \omega_{2}} \rightarrow \mathbb{C}_{p}$. We apply this homomorphism to entries of the matrix $M_{R_{2, \omega_{2}}}$ and get a matrix $M_{\tau_{l_{2}}} \in \mathrm{M}_{\infty}\left(\mathbb{C}_{p}\right)$. Let $R_{2, \omega_{2}} \llbracket P_{J_{2}^{c}}^{\prime} \rrbracket \rightarrow \mathbb{C}_{p}$ be the composite of the homomorphisms $R_{2, \omega_{2}} \llbracket P_{J_{2}^{c}}^{\prime} \rrbracket \rightarrow R_{2, \omega_{2}}$ and $\tau_{l_{2}}$ : $R_{2, \omega_{2}} \rightarrow \mathbb{C}_{p}$. Denote $\mathbb{C}_{p} \llbracket \mathcal{O}_{\mathfrak{p}_{2}} \rrbracket^{j_{2}-\bmod }=R_{2, \omega_{2}} \llbracket \mathcal{O}_{\mathfrak{p}_{2}} \rrbracket^{j_{2}-\text { mod }} \otimes_{R_{2, \omega_{2}}, \tau_{l_{2}}} \mathbb{C}_{p}$. From the explicit expression $V_{\alpha, \omega_{2}}^{\text {mod }} \cong \prod_{k=1}^{d_{\alpha}} R_{2, \omega_{2}} \llbracket \mathcal{O}_{\mathfrak{p}_{2}} \times P_{J_{2}^{c}}^{\prime} \rrbracket^{j_{2}-m o d}$, we have an isomorphism $V_{\alpha, \omega_{2}}^{\text {mod }} \otimes_{R_{2, \omega_{2}} \llbracket P_{J_{2}^{c}}^{\prime} \rrbracket}$ $\mathbb{C}_{p} \cong \prod_{k=1}^{d_{\alpha}} \mathbb{C}_{p} \llbracket \mathcal{O}_{\mathfrak{p}_{2}} \rrbracket^{j_{2}-\text { mod }}$

Under the isomorphism $R_{2, \omega_{2}} \llbracket \mathcal{O}_{\mathfrak{p}_{2}} \rrbracket^{j_{2}-\text { mod }} \cong R_{2, \omega_{2}} \llbracket X_{2}^{\prime} \rrbracket$, the space $\mathbb{C}_{p} \llbracket \mathcal{O}_{\mathfrak{p}_{2}} \rrbracket^{j_{2}-\text { mod }}$ admits a quotient $\mathbb{C}_{p} \llbracket X_{2}^{\prime} \rrbracket^{\text {deg } \leqslant l_{2}}$ consisting of polynomials of $X_{2}^{\prime}$ of degree $\leqslant l_{2}$. This quotient is stable under the action of the monoid $\mathbf{M}_{\pi_{2}}$. 
Recall that $V_{\alpha}^{\text {mod }}$ is a $R_{2, \omega_{2}} \llbracket P_{J_{2}^{c}}^{\prime} \rrbracket$-submodule of $S_{\kappa, J_{2}}^{D, J_{2}}\left(K^{p}, R_{2, \omega_{2}}\right)^{\vee}$, and we have an $R_{2, \omega_{2}} \llbracket P_{J_{2}^{c}}^{\prime} \rrbracket$-linear isomorphism $V_{\alpha}^{m o d} \cong \prod_{k=1}^{d_{\alpha}} R_{2, \omega_{2}} \llbracket \mathcal{O}_{\mathfrak{p}_{2}} \times P_{J_{2}^{c}}^{\prime} \rrbracket j^{j_{2}-m o d}$. We put $V_{\alpha, \mathbb{C}_{p}}^{m o d}:=$ $V_{\alpha}^{m o d} \otimes_{R_{2, \omega_{2}} \llbracket P_{J_{2}^{\prime}}^{\prime} \rrbracket} \mathbb{C}_{p}$ and obtain an isomorphism $V_{\alpha, \mathbb{C}_{p}}^{\bmod } \cong \prod_{k=1}^{d_{\alpha}} \mathbb{C}_{p} \llbracket \mathcal{O}_{\mathfrak{p}_{2}} \rrbracket^{j_{2}-\bmod }$.

Given the locally algebraic weight $\kappa_{l_{2}}$ defined above, we define a subspace $\mathcal{C}\left(\mathcal{O}_{\mathfrak{p}_{2}}, \mathbb{C}_{p}\right)$ of $\mathcal{C}\left(\mathcal{O}_{\mathfrak{p}_{2}}, \mathbb{C}_{p}\right)$ consisting of functions $f$ with the property that $\left.f\right|_{a+\pi_{2} \mathcal{O}_{\mathfrak{p}_{2}}}$ is a polynomial function of degree less or equal to $l_{2}$, for all $a \in \mathcal{O}_{\mathfrak{p}_{2}}$. Then we have $\operatorname{dim}_{\mathbb{C}_{p}} \mathcal{C}\left(\mathcal{O}_{\mathfrak{p}_{2}}, \mathbb{C}_{p}\right)^{c l}=p\left(l_{2}+1\right)$. We use $\mathbb{C}_{p} \llbracket \mathcal{O}_{\mathfrak{p}_{2}} \rrbracket^{c l}$ to denote the $\mathbb{C}_{p}$-dual of $\mathcal{C}\left(\mathcal{O}_{\mathfrak{p}_{2}}, \mathbb{C}_{p}\right)^{c l}$. Then $\mathbb{C}_{p} \llbracket \mathcal{O}_{\mathfrak{p}_{2}} \rrbracket^{c l}$ is a quotient of $\mathbb{C}_{p} \llbracket \mathcal{O}_{\mathfrak{p}_{2}} \rrbracket^{j_{2}-\text { mod }}$ and hence $V_{\alpha, \mathbb{C}_{p}}^{\text {mod }}$ admits a quotient $V_{\alpha, \mathbb{C}_{p}}^{c l}:=\prod_{k=1}^{d_{\alpha}} \mathbb{C}_{p} \llbracket \mathcal{O}_{\mathfrak{p}_{2}} \rrbracket^{c l}$. Moreover, this quotient also carries an action of the $U_{\pi_{1}}$ and $U_{\pi_{2}}$-operators induced from these operators on $V_{\alpha, \mathbb{C}_{p}}^{\text {mod }}$. We define $W_{\alpha, \mathbb{C}_{p}}^{c l}=V_{\alpha, \mathbb{C}_{p}}^{c l} / V_{\gamma, \mathbb{C}_{p}}^{c l}$, which is isomorphic to $\prod_{k=1}^{r_{\alpha}} \mathbb{C}_{p} \llbracket \mathcal{O}_{\mathfrak{p}_{2}} \rrbracket^{c l}$.

We use $V_{\alpha, \mathbb{C}_{p}}^{c l, v}$ to denote the $\mathbb{C}_{p}$-dual of $V_{\alpha, \mathbb{C}_{p}}^{c l}$. From the above construction, we see that there exists $r \in \mathcal{N}^{I}$, such that $V_{\alpha, \mathbb{C}_{p}}^{c l, \vee}$ lies in the following subspace

$$
\left\{\phi: D^{\times} \backslash D_{f}^{\times} / K^{p} \rightarrow \mathcal{C}^{r, a n}\left(\mathcal{O}_{\mathfrak{p}_{1}} \times \mathcal{O}_{\mathfrak{p}_{2}}, \mathbb{C}_{p}\right) \mid \phi(x u)=\phi(x) \circ u, \text { for all } x \in D_{f}^{\times}, u \in \mathrm{Iw}_{\pi^{t}}\right\}
$$

of $S_{\kappa_{l}, I}^{D}\left(K^{p}, \mathbb{C}_{p}\right)$ with the additional properties:

- The slopes of $U_{\pi_{1}}$-operator on $V_{\alpha, \mathbb{C}_{p}}^{c l, \vee}$ is less or equal to $\lambda_{l} \cdot v_{p}\left(\chi_{l_{2}}\left(T_{j}\right)\right)=\frac{\lambda_{l}}{p-1}<l_{2}+1$; and

- $\left.\phi(x)\right|_{\left\{x_{1}\right\} \times \mathcal{O}_{\mathfrak{p}_{2}}}$ is a polynomial function of degree less or equal to $l_{2}$, for all $x_{1} \in \mathcal{O}_{\mathfrak{p}_{1}}$.

Fix a locally algebraic weight $\kappa_{l_{2}}$ as above. For $1 \leqslant l \leqslant g$, we have defined an operator $\theta_{i_{l}}: S_{\kappa_{l_{2}}}^{D}\left(K^{p}, r\right) \rightarrow S_{\kappa_{l_{2}}^{\prime}}^{D}\left(K^{p}, r\right)$ in $\$ 3.8$. It follows from the first property and the fact that the $U_{\pi_{1}}$-slopes on the space $S_{\kappa_{l_{2}}^{\prime}}^{D}\left(K^{p}, r\right)$ are all nonnegative that $V_{\alpha, \mathbb{C}_{p}}^{c l, \vee} \subset \operatorname{ker}\left(\theta_{i_{1}}\right)$. It follows from the second property and the definition of $\theta_{i_{l}}$ 's that $V_{\alpha, \mathbb{C}_{p}}^{c l, \vee} \subset \operatorname{ker}\left(\theta_{i_{l}}\right)$ for $l=2, \ldots, g$. By Proposition 3.8.9, we conclude that $V_{\alpha, \mathbb{C}_{p}}^{c l, \vee} \subset \underset{\omega_{J_{2}^{c}}: \Delta_{J_{2}^{c}} \rightarrow \mathbb{Z}_{p}^{\times}}{\bigoplus} S_{k, w}^{D}\left(K^{p}, \psi\right)$.

We make the same construction starting with another character $\omega_{2}^{\prime}=\left(\eta_{j_{1}}^{-1}, \eta_{j_{2}}^{-1}, \eta\right)$ : $H_{2} \rightarrow \mathbb{Z}_{p}^{\times}$. We obtain $\underline{\lambda}$-Hodge bounded matrices $M_{R_{2, \omega_{J_{2}}}^{\prime}} \in \mathrm{M}_{\infty}\left(R_{2, \omega_{J_{2}}^{\prime}}\right), \bar{M}^{\prime} \in \mathrm{M}_{\infty}\left(\mathbb{F}_{p} \llbracket T_{j} \rrbracket\right)$, and for every $l_{2} \in 2 \mathbb{Z}_{\geqslant 0}$, we get a matrix $M_{\tau_{l_{2}}}^{\prime} \in \mathrm{M}_{\infty}\left(\mathbb{C}_{p}\right)$. We also have spaces $\left(V_{\alpha, \mathbb{C}_{p}}^{\text {mod }}\right)^{\prime}$, $\left(V_{\alpha, \mathbb{C}_{p}}^{c l}\right)^{\prime}$ and $\left(W_{\alpha, \mathbb{C}_{p}}^{c l}\right)^{\prime}$. It follows from Proposition 3.8.5 that the $U_{\pi_{2}}$-slopes on the spaces $W_{\alpha, \mathbb{C}_{p}}^{c l}$ and $\left(W_{\alpha, \mathbb{C}_{p}}^{c l}\right)^{\prime}$ can be paired such that the slopes in each pair sum to $l_{2}-1$. In other words, there exist $r_{\alpha} p\left(l_{2}+1\right)$ pairs of the slopes of the Newton polygons of the matrices $M_{\tau_{l_{2}}}$ and $M_{\tau_{l_{2}}}^{\prime}$, such that the slopes in each pair sum to $l_{2}-1$. It follows from a similar argument in $\S 4.6$ that the first $r_{\alpha} p\left(l_{2}+1\right)$ slopes of the matrix $M_{\tau_{l_{2}}}$ sum to $\frac{1}{2} r_{\alpha} p\left(l_{2}+1\right)$.

We use $\operatorname{char}\left(M_{R_{2, \omega_{2}}}\right)=\sum_{n \geqslant 0} c_{n} X^{n} \in R_{2, \omega_{2}} \llbracket X \rrbracket$ to denote the characteristic power series of the matrix $M_{R_{2, \omega_{2}}}$ with $c_{n}(\underline{T}) \in R_{1, \omega_{1}} \llbracket T_{2} \rrbracket \subset R_{2, \omega_{2}}$ and $\underline{T}=\left(T_{1}, T_{2}, T\right)$. For all $l_{2} \in 2 \mathbb{Z}_{\geqslant 0}$, the Newton polygon of $\sum_{n \geqslant 0} c_{n}\left(\chi_{l_{2}}(\underline{T})\right) X^{n}$ passes through the point $\left(r_{\alpha} p\left(l_{2}+\right.\right.$ 1), $\left.\mu_{r_{\alpha} p\left(l_{2}+1\right)} v_{p}\left(\chi_{l_{2}}\left(T_{2}\right)\right)\right)$. Write $c_{n}(\underline{T})=\sum_{m \geqslant 0} b_{n, m} T_{2}^{m}$ with $b_{n, m} \in R_{1, \omega_{1}}$. Recall that $R_{1, \omega_{1}}$ is isomorphic to $\mathbb{Z}_{p} \llbracket T_{1}, \frac{p}{T_{1}}, T \rrbracket$. In particular, $R_{1, \omega_{1}}$ is a local ring and we use $\mathfrak{m}_{1, \omega_{1}}$ to denote its maximal ideal. For $b\left(T_{1}, T\right) \in R_{1, \omega_{1}}$, the following statements are equivalent: 
(1) $b\left(T_{1}, T\right)$ is a unit in $R_{1, \omega_{1}}$;

(2) $b\left(T_{1}, T\right) \notin \mathfrak{m}_{1, \omega_{1}}$;

(3) there exist $t_{1}, t \in \mathbb{C}_{p}$ with $\left|t_{1}\right|_{p} \in\left(\frac{1}{p}, 1\right)$ and $|t|_{p}<1$, such that $\left|b\left(t_{1}, t\right)\right|_{p}=1$, i.e. $b\left(t_{1}, t\right)$ is a $p$-adic unit.

Now we can run the same argument in $\$ 4.6$ to the matrix $M \in \mathrm{M}_{\infty}\left(R_{2, \omega_{2}} \llbracket P_{J_{2}^{c}}^{\prime} \rrbracket\right), M_{R_{2, \omega_{2}}} \in$ $\mathrm{M}_{\infty}\left(R_{2, \omega_{2}}\right)$ and $\bar{M} \in \mathrm{M}_{\infty}\left(\mathbb{F}_{p} \llbracket T_{2} \rrbracket\right)$. We obtain a set

$$
\Omega_{\omega_{2}, \alpha_{1}}=\left\{n_{\omega_{2}, \alpha_{1}, l}^{-}, n_{\omega_{2}, \alpha_{1}, l}^{+} \mid l \in\{0\} \bigcup 1+2 \mathbb{Z}_{\geqslant 0}\right\}
$$

and a filtration $\left\{\tilde{V}_{\alpha_{1}, \alpha_{2}} \mid \alpha_{2} \in \Omega_{\omega_{2}, \alpha_{1}}\right\}$ of the graded piece $W_{\alpha_{1}, \omega_{2}}^{m o d}$ as a left $R_{2, \omega_{2}} \llbracket P_{J_{2}^{c}}^{\prime} \rrbracket$-module.

Fix a character $\omega: H \rightarrow \mathbb{Z}_{p}^{\times}$. For $1 \leqslant l \leqslant g$, we use $\omega_{l}: H_{l} \rightarrow \mathbb{Z}_{p}^{\times}$to denote the restriction of $\omega$ to $H_{l}$.

Now we apply the above construction inductively to the places $j_{3}, \ldots, j_{g}$. In summary, we get the following datum:

(1) There exists a set $\Omega_{\omega_{1}}=\left\{n_{\omega_{1}, l}^{-}, n_{\omega_{1}, l}^{+} \mid l \in\{0\} \cup 1+2 \mathbb{Z}_{\geqslant 0}\right\}$ and a filtration $\left\{F_{\alpha_{1}} \mid \alpha_{1} \in\right.$ $\left.\Omega_{\omega_{1}}\right\}$ of $S_{\kappa, I}^{D, I}\left(K^{p}, \Lambda_{\omega}^{>1 / p}\right)^{\vee}$ consisting of free $\Lambda_{\omega}^{>1 / p}$-modules. We use $\left\{G_{\alpha_{1}} \mid \alpha_{1} \in \Omega_{\omega_{1}}\right\}$ to denote the graded pieces of this filtration, i.e. $G_{\alpha_{1}}=F_{\alpha_{1}} / F_{\gamma_{1}}$, if $\gamma_{1} \leqslant \alpha_{1}$ are two consecutive elements in $\Omega_{1}$ (we denote $G_{n_{\omega_{1}, 0}^{-}}=F_{n_{\omega_{1}, 0}^{-}}=(0)$ ).

(2) For any $\alpha_{1} \in \Omega_{\omega_{1}}$, there exist a set $\Omega_{\omega_{2}, \alpha_{1}}=\left\{n_{\omega_{2}, \alpha_{1}, l}^{-}, n_{\omega_{2}, \alpha_{1}, l}^{+} \mid l \in\{0\} \cup 1+2 \mathbb{Z}_{\geqslant 0}\right\}$ and a filtration $\left\{F_{\alpha_{1}, \alpha_{2}} \mid \alpha_{2} \in \Omega_{\omega_{2}, \alpha_{1}}\right\}$ of $G_{\alpha_{1}}$ consisting of free $\Lambda_{\omega}^{>1 / p}$-modules; define $G_{\alpha_{1}, \alpha_{2}}=F_{\alpha_{1}, \alpha_{2}} / F_{\alpha_{1}, \gamma_{2}}$ if $\gamma_{2} \leqslant \alpha_{2}$ are two consecutive elements in $\Omega_{\omega_{2}, \alpha_{1}}$.

(3) For $2 \leqslant k \leqslant g-1$, given the set $\Omega_{\omega_{k}, \alpha_{1}, \ldots, \alpha_{k-1}}$ and the space $G_{\alpha_{1}, \ldots, \alpha_{k}}$, there exist a set $\Omega_{\omega_{k+1}, \alpha_{1}, \ldots, \alpha_{k}}=\left\{n_{\omega_{k+1}, \alpha_{1}, \ldots, \alpha_{k}, l}^{-}, n_{\omega_{k+1}, \alpha_{1}, \ldots, \alpha_{k}, l}^{+} \mid l \in\{0\} \cup 1+2 \mathbb{Z}_{\geqslant 0}\right\}$ and a filtration $\left\{F_{\alpha_{1}, \ldots, \alpha_{k+1}} \mid \alpha_{k+1} \in \Omega_{\omega_{k+1}, \alpha_{1}, \ldots, \alpha_{k}}\right\}$ of $G_{\alpha_{1}, \ldots, \alpha_{k}}$ consisting of free $\Lambda_{\omega}^{>1 / p}$-modules; define $G_{\alpha_{1}, \ldots, \alpha_{k+1}}=F_{\alpha_{1}, \ldots, \alpha_{k+1}} / F_{\alpha_{1}, \ldots, \gamma_{k+1}}$ if $\gamma_{k+1} \leqslant \alpha_{k+1}$ are two consecutive elements in $\Omega_{\omega_{k+1}, \alpha_{1}, \ldots, \alpha_{k}}$.

Moreover, we have the following properties of the above filtrations:

(1) all the filtrations are stable under the $U_{\pi_{i}}$-operators, for all $i \in I$; and

(2) the graded pieces $G_{\alpha_{1}, \ldots, \alpha_{g}}$ obtained above are free $\Lambda_{\omega}^{>1 / p}$-modules of finite rank (possibly 0).

5.3. Conclusion. For any $x \in \mathcal{W}_{\omega}^{>1 / p}\left(\mathbb{C}_{p}\right)$, we use $\chi_{x}: \Lambda_{\omega}^{>1 / p} \rightarrow \mathbb{C}_{p}$ to denote the corresponding homomorphism. For any free $\Lambda_{\omega}^{>1 / p}$-module $G_{\alpha_{1}, \ldots, \alpha_{g}}$ we obtained in the previous section, we denote $G_{\alpha_{1}, \ldots, \alpha_{g}}(x)=G_{\alpha_{1}, \ldots, \alpha_{g}} \otimes_{\Lambda_{\omega}^{>1 / p}, \chi_{x}} \mathbb{C}_{p}$.

Proposition 5.3.1. For all $1 \leqslant k \leqslant g$, if $\alpha_{k}=n_{\omega_{J_{k}}, \alpha_{1} \ldots, \alpha_{k-1}, l}^{-}$for some $l \in\{0\} \cup 1+$ $2 \mathbb{Z}_{\geqslant 0}$, the slopes of the $U_{\pi_{k}}$-operator on $G_{\alpha_{1}, \ldots, \alpha_{g}}(x)$ are all equal to $(p-1) v_{p}\left(\chi_{x}\left(T_{i_{k}}\right)\right) \cdot l$; if $\alpha_{k}=n_{\omega_{J_{k}}, \alpha_{1} \ldots, \alpha_{k-1}, l}^{+}$for some $l \in\{0\} \cup 1+2 \mathbb{Z}_{\geqslant 0}$, the slopes of the $U_{\pi_{k}}$-operator on $G_{\alpha_{1}, \ldots, \alpha_{g}}(x)$ all belong to the interval $(p-1) v_{p}\left(\chi_{x}\left(T_{i_{k}}\right)\right) \cdot(l, l+2)$ if $l \neq 0$, and the interval $(p-1) v_{p}\left(\chi_{x}\left(T_{i_{k}}\right)\right) \cdot(0,1)$ if $l=0$.

Proof. We give a proof for $l=1$. The argument for other $l$ 's is similar. 
Recall that by Proposition 4.6.1, we have a filtration $\left\{\tilde{V}_{\alpha_{1}} \mid \alpha_{1} \in \Omega_{\omega_{1}}\right\}$ of the space $S_{\kappa_{1}, J_{1}}^{D, J_{1}}\left(K^{p}, R_{1, \omega_{1}}\right)^{\vee}$. We have an isomorphism $\tilde{V}_{\alpha_{1}} \cong \prod_{k=1}^{d_{\alpha_{1}}} R_{1, \omega_{1}} \llbracket P_{J_{1}^{c}}^{\prime} \rrbracket$ of left $R_{1, \omega_{1}} \llbracket P_{J_{1}^{c}}^{\prime} \rrbracket-$ modules for all $\alpha_{1} \in \Omega_{\omega_{1}}$. These isomorphisms induces isomorphisms $\tilde{W}_{\alpha_{1}}:=\tilde{V}_{\gamma_{1}} / \tilde{V}_{\alpha_{1}} \cong$ $\prod_{k=1}^{r_{\alpha_{1}}} R_{1, \omega_{1}} \llbracket P_{J_{1}^{c}}^{\prime} \rrbracket$.

Fix $\alpha_{1} \in \Omega_{\omega_{1}}$ and let $\gamma_{1} \in \Omega_{\omega_{1}}$ such that $\alpha_{1} \leqslant \gamma_{1}$ are two consecutive elements in $\Omega_{\omega_{1}}$. Under the above isomorphisms, the $U_{\pi_{1}}$-operator on $\tilde{W}_{\alpha_{1}}$ corresponds to a matrix

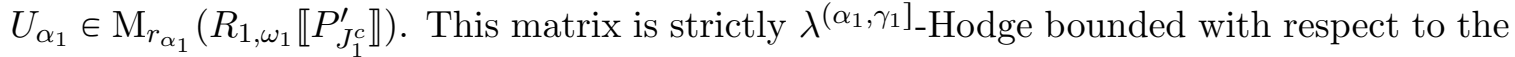
element $T_{1} \in R_{1, \omega_{1}} \llbracket P_{J_{1}^{c}}^{\prime} \rrbracket$.

First we assume that $\alpha_{1}=n_{\omega_{1}, l}^{-}$for some $l \in\{0\} \cup 1+2 \mathbb{Z}_{\geqslant 0}$. We can assume that $\alpha_{1} \neq \gamma_{1}$, or otherwise $G_{\alpha_{1}, \ldots, \alpha_{g}}=0$ and the proposition is trivial in this case. From the construction in Proposition 4.6.1, we see that $\lambda_{\alpha_{1}+1}=\lambda_{\gamma_{1}}=(p-1) l$. Hence there exists an invertible matrix $U_{\alpha_{1}}^{\prime} \in \mathrm{GL}_{r_{\alpha_{1}}}\left(R_{1, \omega_{1}} \llbracket P_{J_{1}^{c}}^{\prime} \rrbracket\right)$, such that $U_{\alpha_{1}}=T_{1}^{(p-1) l} U_{\alpha_{1}}^{\prime}$. Under the isomorphism $\tilde{W}_{\alpha_{1}} \cong \prod_{k=1}^{r_{\alpha_{1}}} R_{1, \omega_{J_{1}}} \llbracket P_{J_{1}^{c}}^{\prime} \rrbracket$, the matrix $U_{\alpha_{1}}^{\prime}$ defines an invertible $R_{1, \omega_{J_{1}}} \llbracket P_{J_{1}^{c}}^{\prime} \rrbracket$-linear operator $U_{\pi_{1}}^{\prime}$ on $\tilde{W}_{\alpha_{1}}$. Recall that $V_{\alpha_{1}}$ is the image of $\tilde{V} \otimes_{R_{1, \omega_{1}}} R_{2, \omega_{2}}$ under the map $S_{\kappa, J_{1}}^{D, J_{1}}\left(K^{p}, R_{2, \omega_{2}}\right)^{\vee} \rightarrow$ $S_{\kappa, J_{2}}^{D, J_{1}}\left(K^{p}, R_{2, \omega_{2}}\right)^{\vee}$. Hence we have isomorphisms $V_{\alpha_{1}} \cong \prod_{k=1}^{d_{\alpha_{1}}} R_{2, \omega_{2}} \llbracket \mathcal{O}_{\mathfrak{p}_{2}} \times P_{J_{2}^{c}}^{\prime} \rrbracket$ and $V_{\alpha_{1}}^{\text {mod }} \cong$ $\prod_{k=1}^{d_{\alpha_{1}}} R_{2, \omega_{2}} \llbracket \mathcal{O}_{\mathfrak{p}_{2}} \times P_{J_{2}^{c}}^{\prime} \rrbracket^{j_{2}-m o d}$. It induces isomorphisms $W_{\alpha_{1}} \cong \prod_{k=1}^{r_{\alpha_{1}}} R_{2, \omega_{2}} \llbracket \mathcal{O}_{\mathfrak{p}_{2}} \times P_{J_{2}^{c}}^{\prime} \rrbracket$ and $W_{\alpha_{1}}^{\text {mod }} \cong \prod_{k=1}^{r_{\alpha_{1}}} R_{2, \omega_{2}} \llbracket \mathcal{O}_{\mathfrak{p}_{2}} \times P_{J_{2}^{c}}^{\prime} \rrbracket^{j_{2}-\text { mod }}$. Under these isomorphisms, the matrix $U_{\alpha_{1}}^{\prime}$ defines invertible operators $U_{\pi_{1}}^{\prime}$ on $W_{\alpha_{1}}$ and $W_{\alpha_{1}}^{\text {mod }}$, but notice that these operators are only $R_{2, \omega_{2}} \llbracket P_{J_{2}^{c}}^{\prime} \rrbracket$-linear. Moreover, the operator $U_{\pi_{1}}^{\prime}$ preserves the filtrations $\left\{W_{\alpha_{1}, \alpha_{2}}^{\text {mod }} \mid \alpha_{2} \in \Omega_{\omega_{2}, \alpha_{1}}\right\}$ as the operator $U_{\pi_{1}}$ does. We apply the above argument inductively to the places $i_{2}, \ldots, i_{g}$ and conclude that there is an operator $U_{\pi_{1}}^{\prime}$ on the graded pieces $G_{\alpha_{1}, \ldots, \alpha_{g}}$ such that $U_{\pi_{1}}^{\prime}$ is invertible and $\Lambda_{\omega}^{>1 / p}$-linear, and satisfies $U_{\pi_{1}}=T_{1}^{(p-1) l} U_{\pi_{1}}^{\prime}$.

Notice that the homomorphism $\chi_{x}: \Lambda_{\omega}^{>1 / p} \rightarrow \mathbb{C}_{p}$ factors through $\mathcal{O}_{\mathbb{C}_{p}}$ and hence $G_{\alpha_{1}, \ldots, \alpha_{g}}(x)$ admits a lattice $L_{\alpha_{1}, \ldots, \alpha_{g}}(x)=G_{\alpha_{1}, \ldots, \alpha_{g}} \otimes_{\Lambda_{\omega}^{>1 / p}, \chi_{x}} \mathcal{O}_{\mathbb{C}_{p}}$. Both the operators $U_{\pi_{1}}$ and $U_{\pi_{1}}^{\prime}$ preserve this lattice. Since $U_{\pi_{1}}^{\prime}$ is invertible on $L_{\alpha_{1}, \ldots, \alpha_{g}}(x)$, the slopes of $U_{\pi_{1}}^{\prime}$ are all 0 . So the slopes of $U_{\pi_{1}}$ on $G_{\alpha_{1}, \ldots, \alpha_{g}}(x)$ are all equal to $(p-1) v_{p}\left(\chi_{x}\left(\left(T_{1}\right)\right)\right) \cdot l$.

Now we assume that $\alpha_{1}=n_{\omega_{1}, l}^{+}$for some $l \in\{0\} \cup 1+2 \mathbb{Z}_{\geqslant 0}$. As in the first case, we can find a matrix $U_{\alpha_{1}}^{\prime} \in \mathrm{M}_{r_{\alpha_{1}}}\left(R_{1, \omega_{1}} \llbracket P_{J_{1}^{c}}^{\prime} \rrbracket\right)$, such that $U_{\alpha_{1}}=T_{1}^{(p-1) l} U_{\alpha_{1}}^{\prime}$, but this matrix is not invertible in general. It follows that there exists a $\Lambda_{\omega}^{>1 / p}$-linear operator $U_{\pi_{1}}^{\prime}$ on $G_{\alpha_{1}, \ldots, \alpha_{g}}$, such that $U_{\pi_{1}}=T_{1}^{(p-1) l} U_{\pi_{1}}^{\prime}$. Since $U_{\pi_{1}}^{\prime}$ preserves the lattice $L_{\alpha_{1}, \ldots, \alpha_{g}}(x)$, the slopes of $U_{\pi_{1}}^{\prime}$ on $G_{\alpha_{1}, \ldots, \alpha_{g}}(x)$ are all nonnegative. So the slopes of $U_{\pi_{1}}$ on $G_{\alpha_{1}, \ldots, \alpha_{g}}(x)$ are all no less than $(p-1) v_{p}\left(\chi_{x}\left(\left(T_{1}\right)\right)\right) \cdot l$.

Recall that $R_{1, \omega_{1}}$ is isomorphic to $\mathbb{Z}_{p} \llbracket T_{1}, \frac{p}{T_{1}}, T \rrbracket$. In particular, it is a local ring with residue field $\mathbb{F}_{p}$. Hence we have a natural surjective map $R_{1, \omega_{1}} \rightarrow \mathbb{F}_{p}$. We apply the homomorphisms $R_{1, \omega_{1}} \llbracket P_{J_{1}^{c}}^{\prime} \rrbracket \rightarrow \mathbb{F}_{p} \llbracket P_{J_{1}^{c}}^{\prime} \rrbracket$ and $\mathbb{F}_{p} \llbracket P_{J_{1}^{c}}^{\prime} \rrbracket \rightarrow \mathbb{F}_{p}$ to entries of the matrix $U_{\alpha_{1}}^{\prime} \in$ $\mathrm{M}_{r_{\alpha_{1}}}\left(R_{1, \omega_{1}} \llbracket P_{J_{1}^{c}}^{\prime} \rrbracket\right)$, and get matrices $U_{\alpha_{1}, \mathbb{F}_{p} \llbracket P_{J_{1}^{c}}^{\prime} \rrbracket}^{\prime} \in \mathrm{M}_{r_{\alpha_{1}}}\left(\mathbb{F}_{p} \llbracket P_{J_{1}^{c}}^{\prime} \rrbracket\right)$ and $U_{\alpha_{1}, \mathbb{F}_{p}}^{\prime} \in \mathrm{M}_{r_{\alpha_{1}}}\left(\mathbb{F}_{p}\right)$. By Proposition 4.6.1, the slopes of the matrix $\bar{U}_{\alpha_{1}} \in \mathrm{M}_{r_{\alpha_{1}}}\left(\mathbb{F}_{p} \llbracket T_{1} \rrbracket\right)$ (with respect to the $T_{1}$-adic valuation) all belong to the interval $\left(\lambda_{\alpha_{1}}, \lambda_{\gamma_{1}}\right)=((p-1) l,(p-1)(l+2))$. So the slopes of 
$\bar{U}_{\alpha_{1}}^{\prime} \in \mathrm{M}_{r_{\alpha_{1}}}\left(\mathbb{F}_{p} \llbracket T_{1} \rrbracket\right)$ are all positive. If we use $f(X) \in \mathbb{F}_{p}[X]$ to denote the characteristic polynomial of the matrix $U_{\alpha_{1}, \mathbb{F}_{p}}^{\prime}$, then we have $f(X)=X^{r_{\alpha_{1}}}$.

We use $\mathcal{I} \subset \mathbb{F}_{p} \llbracket P_{J_{1}^{c}}^{\prime} \rrbracket$ to denote the augmentation ideal of this complete group ring. It follows from Hamilton-Cayley Theorem that $f\left(U_{\alpha_{1}, \mathbb{F}_{p}}^{\prime}\right)=\left(U_{\alpha_{1}, \mathbb{F}_{p}}^{\prime}\right)^{r_{\alpha_{1}}}=0$, and hence



Define $\bar{L}_{\alpha_{1}, \ldots, \alpha_{g}}(x)=L_{\alpha_{1}, \ldots, \alpha_{g}}(x) \otimes_{\mathcal{O}_{\mathbb{C}_{p}}} \overline{\mathbb{F}}_{p}$. It is a finite $\overline{\mathbb{F}}_{p^{-v}}$-vector space and $\mathrm{Iw}_{\pi}$ acts on it continuously. It follows that the action of $\mathcal{I}$ on $\bar{L}_{\alpha_{1}, \ldots, \alpha_{g}}(x)$ is nilpotent and hence $U_{\alpha_{1}, \mathbb{F}_{p} \llbracket P_{J_{1}^{c}}^{\prime} \rrbracket}$ is a nilpotent operator on $\bar{L}_{\alpha_{1}, \ldots, \alpha_{g}}(x)$. So the slopes of the $U_{\pi_{1}}^{\prime}$-operator on $G_{\alpha_{1}, \ldots, \alpha_{g}}(x)$ are all positive, and we conclude that the slopes of the $U_{\pi_{1}}^{\prime}$-operator on $G_{\alpha_{1}, \ldots, \alpha_{g}}(x)$ are all strictly larger than $(p-1) v_{p}\left(\chi_{x}\left(\left(T_{1}\right)\right)\right) \cdot l$.

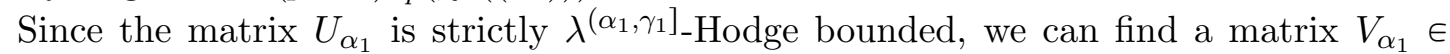
$\mathrm{M}_{r_{\alpha_{1}}}\left(R_{1, \omega_{J_{1}}} \llbracket P_{J_{1}^{c}}^{\prime} \rrbracket\right)$, such that $U_{\alpha_{1}} V_{\alpha_{1}}=V_{\alpha_{1}} U_{\alpha_{1}}=T_{1}^{(p-1)(l+2)} \cdot I_{r_{\alpha_{1}}}$.

The matrix $V_{\alpha_{1}}$ induces a $\Lambda_{\omega}^{>1 / p}$-linear operator $V_{\pi_{1}}$ on $G_{\alpha_{1}, \ldots, \alpha_{g}}$ and $G_{\alpha_{1}, \ldots, \alpha_{g}}(x)$. It preserves the lattice $L_{\alpha_{1}, \ldots, \alpha_{g}}(x)$ and satisfies $U_{\pi_{1}} V_{\pi_{1}}=V_{\pi_{1}} U_{\pi_{1}}=T_{1}^{(p-1)(l+2)} \cdot$ Id. It follows from Lemma 3.8.6 that the slopes of $U_{\pi_{1}}$ and $V_{\pi_{1}}$ on $G_{\alpha_{1}, \ldots, \alpha_{g}}(x)$ can be paired such that the slopes in each pair sum to $(p-1) v_{p}\left(\chi_{x}\left(\left(T_{1}\right)\right)\right) \cdot(l+2)$. We have seen that the slopes of $\bar{U}_{\alpha_{1}} \in \mathrm{M}_{r_{\alpha_{1}}}\left(\mathbb{F}_{p} \llbracket T_{1} \rrbracket\right)$ all belong to the interval $((p-1) l,(p-1)(l+2))$. Hence the slopes of $\bar{V}_{\alpha_{1}}$ are all positive. By a similar argument as above, we can show that the slopes of $V_{\pi_{1}}$ on $G_{\alpha_{1}, \ldots, \alpha_{g}}(x)$ are all positive. It follows that the slopes of $U_{\pi_{1}}$ on $G_{\alpha_{1}, \ldots, \alpha_{g}}(x)$ are all strictly less than $(p-1) v_{p}\left(\chi_{x}\left(\left(T_{1}\right)\right)\right) \cdot(l+2)$.

Now we are ready to prove our main theorem 1.0.2. Let $X=\operatorname{Sp}(A) \subset \mathcal{W}_{\omega}^{>1 / p}$ be an affinoid subdomain that corresponds to the continuous homomorphism $\chi: \Lambda_{\omega}^{>1 / p} \rightarrow A$. For $l \in$ $\{1, \ldots, g\}$ and a set of indices $\left\{\alpha_{k} \mid k=1, \ldots, l\right\}$ with $\alpha_{1} \in \Omega_{\omega_{1}}, \alpha_{k+1} \in \Omega_{\omega_{k+1}, \alpha_{1}, \ldots, \alpha_{k}}$ for $k=$ $1, \ldots, l-1$, we define $F_{\alpha_{1}, \ldots, \alpha_{l}, A}:=F_{\alpha_{1}, \ldots, \alpha_{l}} \hat{\otimes}_{\Lambda_{\omega}^{>1 / p}, \chi} A$ and $G_{\alpha_{1}, \ldots, \alpha_{l}, A}:=G_{\alpha_{1}, \ldots, \alpha_{l}} \hat{\otimes}_{\Lambda_{\omega}^{>1 / p}, \chi} A$. Let $S_{A}:=S_{\kappa, I}^{D, I}\left(K^{p}, \Lambda_{\omega}^{>1 / p}\right) \hat{\otimes}_{\Lambda_{\omega}^{>1 / p}, \chi} A$ and $\psi_{A}: \mathbf{T} \rightarrow \operatorname{End}_{A}\left(S_{A}\right)$ be the natural homomorphism, where $\mathbf{T}$ is the (abstract) $\mathbb{Q}_{p}$-Hecke algebra defined in $\$ 3.6$. Let $\mathbf{T}_{A}$ be the image of $\psi_{A}$. Under the above notations and by our previous construction in $\$ 5.2$, we obtain a filtration $\left\{F_{\alpha_{1}} \mid \alpha_{1} \in \Omega_{\omega_{1}}\right\}$ of the $A$-Banach module $S_{A}$ and the Hecke operators on $S_{A}$ all preserve this filtration. It follows from Proposition 5.3.1 that the induced homomorphism $\mathbf{T}_{A} \rightarrow \prod_{\alpha_{1} \in \Omega_{\omega_{1}}} \operatorname{End}_{A}\left(G_{\alpha_{1}}\right)$ is injective. We inductively run the above argument, and obtain an injective homomorphism $\mathbf{T}_{A} \rightarrow \prod_{\alpha_{1}, \ldots, \alpha_{g}} \operatorname{End}_{A}\left(G_{\alpha_{1}, \ldots, \alpha_{g}}\right)$. The main theorem 1.0 .2 follows from the injectivity of this map, the construction of the eigenvariety $\mathcal{X}_{D}$, and Proposition 5.3 .1 .

\section{Application to Hilbert modular eigenvarieties}

\section{1. p-adic Langlands Functoriality.}

Definition 6.1.1. An eigenvariety datum is a tuple $\mathfrak{D}=(\mathcal{W}, \mathcal{Z}, \mathcal{M}, \mathbf{T}, \psi)$, where $\mathcal{W}$ is a separated reduced equidimensional, relatively factorial (see $[12, \S 4.1]$ for the precise definition) rigid analytic space, $\mathcal{Z} \subset \mathcal{W} \times \mathbb{A}^{1}$ is a Fredholm hypersurface, $\mathcal{M}$ is a coherent analytic sheaf over $\mathcal{Z}, \mathbf{T}$ is a commutative $\mathbb{Q}_{p}$-algebra and $\psi$ is a $\mathbb{Q}_{p}$-algebra homomorphism $\psi: \mathbf{T} \rightarrow \operatorname{End}_{\mathcal{O}_{\mathcal{Z}}}(\mathcal{M})$.

Remark 6.1.2. In $\$ 3.6$, we construct a quasi-coherent sheaf $\mathcal{M}$ on $\mathcal{W}$ coming from the spaces of of overconvergent automorphic forms for $D$. We can also construct a coherent sheaf 
$\mathcal{M}^{*}$ over the spectral variety $\mathcal{Z}_{D}$ coming from the spaces $S_{\kappa}^{D}\left(K^{p} \operatorname{Iw}_{\pi}, r\right)$ 's. The construction need a special admissible cover of $\mathcal{Z}_{D}$ which consists of slope adapted affinoids of $\mathcal{Z}_{D}$. We refer to [5, Chapter 4] and [12, §4] for the details of the construction.

Given an eigenvariety datum $\mathfrak{D}$ as above, we use $\mathcal{X}=\mathcal{X}(\mathfrak{D})$ to denote the eigenvariety associated to $\mathfrak{D}$, and we have a finite morphism $\pi: \mathcal{X} \rightarrow \mathcal{Z}$ and a morphism $w: \mathcal{X} \rightarrow \mathcal{W}$. Recall that in [12, Definition 5.1.1], the core $\mathcal{X}^{\circ}$ of $\mathcal{W}$ is defined to be the union of $\operatorname{dim} \mathcal{W}$ dimensional irreducible components of the nilreduction $\mathcal{X}^{\text {red }}$, and $\mathcal{X}$ is unmixed if $\mathcal{X}^{\circ} \cong \mathcal{X}$.

Remark 6.1.3. The eigenvarieties we will consider in this section are the eigenvarieties $\mathcal{X}_{D}$ constructed in $\$ 3.6$ and Hilbert modular eigenvarieties $\mathcal{X}_{\mathrm{GL}_{2 / F}}$ constructed in $\left.1, \S 5\right]$. These eigenvarieties are unmixed by [2, Theorem 2]. Hence we assume that all the eigenvarieties are unmixed in the rest of this paper.

Given a point $z \in \mathcal{Z}$ and any $T \in \mathbf{T}$, we write $D(T, X)(z) \in k(z)[X]$ for the characteristic polynomial $\left.\operatorname{det}(1-\psi(T) X)\right|_{\mathcal{M}(z)}$.

Now we can state Hansen's interpolation theorem ( [12, Theorem 5.1.6]), which is the main tool to translate our results to Hilbert modular eigenvarieties.

Theorem 6.1.4. Given two eigenvariety data $\mathfrak{D}_{i}=\left(\mathcal{W}_{i}, \mathcal{Z}_{i}, \mathcal{M}_{i}, \mathbf{T}_{i}, \psi_{i}\right)$, let $\mathcal{X}_{i}=\mathcal{X}\left(\mathfrak{D}_{i}\right)$ be the associated eigenvariety for $i=1,2$. Suppose that we are given the following additional data:

(1) a closed immersion $\mathrm{J}: \mathcal{W}_{1} \rightarrow \mathcal{W}_{2}$ and we use $j$ to denote the closed immersion $\jmath \times$ id $: \mathcal{W}_{1} \times \mathbb{A}^{1} \rightarrow \mathcal{W}_{2} \times \mathbb{A}^{1}$

(2) a homomorphism of $\mathbb{Q}_{p}$-algebras $\sigma: \mathbf{T}_{2} \rightarrow \mathbf{T}_{1}$;

(3) a very Zariski dense set $\mathcal{Z}_{1}^{\mathrm{cl}} \subset \mathcal{Z}_{1}$ with $j\left(\mathcal{Z}_{1}^{\mathrm{cl}}\right) \subset \mathcal{Z}_{2}$, such that $D(\sigma(T), X)(z)$ divides $D(T, X)(j(z))$ in $k(z)[X]$ for all $z \in \mathcal{Z}_{1}^{\text {cl }}$ and all $T \in \mathbf{T}_{2}$.

Then there exists a morphism $i: \mathcal{X}_{1} \rightarrow \mathcal{X}_{2}$ with the commutative diagrams

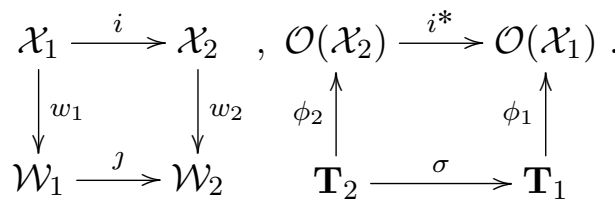

Moreover, $i$ is a composite of a finite morphism followed by a closed immersion.

Remark 6.1.5. When $\mathcal{W}_{1}=\mathcal{W}_{2}=\mathcal{W}, \mathbf{T}_{1}=\mathbf{T}_{2}=\mathbf{T}$ and $\jmath: \mathcal{W}_{1} \rightarrow \mathcal{W}_{2}$ and $\sigma: \mathbf{T}_{2} \rightarrow \mathbf{T}_{1}$ are the identity maps, the map $i: \mathcal{X}_{1} \rightarrow \mathcal{X}_{2}$ in the above theorem is a closed immersion.

6.2. Application to Hilbert modular eigenvarieties. Recall that $F$ is a totally real field in which $p$ splits and $D$ is a totally definite quaternion algebra over $F$ with discriminant $\mathfrak{d}$. We assume that $(p, \mathfrak{d})=1$.

Fix a prime ideal $\mathfrak{n}$ of $F$ p prime to $\mathfrak{d} p$. Set

$$
U_{1}(\mathfrak{n}):=\left\{\gamma \in \mathrm{GL}_{2}\left(\mathcal{O}_{F} \otimes \hat{\mathbb{Z}}\right) \mid \gamma \equiv\left(\begin{array}{cc}
* & * \\
0 & 1
\end{array}\right) \quad \bmod \mathfrak{n}\right\} .
$$

When $\mathfrak{n}$ is prime to $\mathfrak{d}$, we set

$$
U_{1}^{D}(\mathfrak{n}):=\left\{\gamma \in\left(\mathcal{O}_{D} \otimes \hat{\mathbb{Z}}\right)^{\times} \mid \gamma \equiv\left(\begin{array}{cc}
* & * \\
0 & 1
\end{array}\right) \quad \bmod \mathfrak{n}\right\} .
$$

Let $\mathfrak{D}_{1}=\left(\mathcal{W}, \mathcal{Z}_{D}, \mathcal{M}_{D}, \mathbf{T}, \psi_{D}\right)$ be the eigenvariety datum associated to the spaces of overconvergent automorphic forms of tame level $U_{1}^{D}(\mathfrak{n})$ as constructed in \$3.6. We use $\mathcal{X}_{D}\left(U_{1}(\mathfrak{n})\right)$ to denote the corresponding eigenvariety. Let $\mathfrak{D}_{2}=(\mathcal{W}, \mathcal{Z}, \mathcal{M}, \mathbf{T}, \psi)$ be the eigenvariety datum associated to the spaces of overconvergent cuspidal Hilbert modular 
forms of tame level $U_{1}(\mathfrak{n})$ as constructed in $\left[1 \S 5\right.$ and let $\mathcal{X}_{\mathrm{GL}_{2 / F}}\left(U_{1}(\mathfrak{n})\right)$ be the corresponding eigenvariety.

We define $\mathcal{Z}_{D}^{c l} \subset \mathcal{Z}_{D}\left(\mathbb{C}_{p}\right) \subset\left(\mathcal{W} \times \mathbb{A}^{1}\right)\left(\mathbb{C}_{p}\right)$ to be the set of points $z=\left(\chi, \alpha^{-1}\right)$ consisting of a classical weight $\chi \in \mathcal{W}\left(\mathbb{C}_{p}\right)$ corresponding to $\left(v=\left(v_{i}\right) \in \mathbb{Z}^{I}, r \in \mathbb{Z}\right)$ and $\alpha \in \mathbb{C}_{p}^{*}$ with $v_{p}(\alpha)<\min _{i \in I}\left\{n_{i}+1\right\}$. Then it follows from the proof of [8, Proposition 3.5] or the proof of 2 , Proposition 6.7] that the set $\mathcal{Z}_{D}^{c l}$ is very Zariski dense in $\mathcal{Z}_{D}$. From the classicality theorem for overconvergent automorphic forms for $D$ ( [26, Theorem 2.3]) and for overconvergent Hilbert modular forms ( $[25$, Theorem 1]), we have

$$
\mathcal{M}_{D}(z)=S_{k, w}^{D}\left(U_{1}^{D}(\mathfrak{n} \pi)\right)^{U_{\pi}=\alpha} \text {, and } \mathcal{M}(z)=S_{k, w}\left(U_{1}(\mathfrak{n} \mathfrak{d} \pi)\right)^{U_{\pi}=\alpha} .
$$

Now we can apply Theorem 6.1.4 to the eigenvariety data $\mathfrak{D}_{1}$ and $\mathfrak{D}_{2}$ together with the additional data id $: \mathcal{W} \rightarrow \mathcal{W}$ and id $: \mathbf{T} \rightarrow \mathbf{T}$, and get the following theorem.

Theorem 6.2.1. ([2, Theorem 1]) There is a closed immersion $i_{D}: \mathcal{X}_{D}(\mathfrak{n}) \rightarrow \mathcal{X}_{\mathrm{GL}_{2 / F}}(\mathfrak{n} \mathfrak{d})$ interpolating the Jacquet-Langlands correspondence on non-critical classical points. Moreover, when $g=[F: \mathbb{Q}]$ is even, one can choose $D$ with $\mathfrak{d}=1$ so that $i_{D}$ is an isomorphism.

When $g=[F: \mathbb{Q}]$ is even, we can choose $D$ with $\mathfrak{d}=1$ and use the above theorem to translate our main theorem 1.0 .2 to the Hilbert modular eigenvarieties. When $g$ is odd, the discriminant $\mathfrak{d}$ cannot be 1 and the immersion $i_{D}$ in Theorem 6.2.1 is not surjective. We need more work to get the desired results for Hilbert modular eigenvarieties.

We choose a quadratic extension $F^{\prime} / F$, such that $F^{\prime}$ is totally real and $p$ splits completely in $F^{\prime}$. Let $\mathfrak{D}_{3}=\left(\mathcal{W}^{\prime}, \mathcal{Z}^{\prime}, \mathcal{M}^{\prime}, \mathbf{T}^{\prime}, \psi^{\prime}\right)$ be the eigenvariety datum associated to the spaces of overconvergent Hilbert modular forms of tame level $U_{1}^{\prime}\left(\mathfrak{n}^{\prime}\right)$, where $\mathfrak{n}^{\prime}=\mathfrak{n} \mathcal{O}_{F^{\prime}}$ and

$$
U_{1}^{\prime}\left(\mathfrak{n}^{\prime}\right)=\left\{\gamma \in \mathrm{GL}_{2}\left(\mathcal{O}_{G^{\prime}} \otimes \hat{\mathbb{Z}}\right) \mid \gamma \equiv\left(\begin{array}{cc}
* & * \\
0 & 1
\end{array}\right) \quad \bmod \mathfrak{n}^{\prime}\right\}
$$

Let $\mathcal{O}_{p}^{\prime}:=\mathcal{O}_{F^{\prime}} \otimes \mathbb{Z}_{p}$. The norm map $\mathrm{Nm}_{F^{\prime} / F}: \mathcal{O}_{p}^{\prime \times} \rightarrow \mathcal{O}_{p}^{\times}$induces a continuous homomorphism of the completed group rings: $\phi: \mathbb{Z}_{p} \llbracket \mathcal{O}_{p}^{\prime \times} \times \mathbb{Z}_{p}^{\times} \rrbracket \rightarrow \mathbb{Z}_{p} \llbracket \mathcal{O}_{p}^{\times} \times \mathbb{Z}_{p}^{\times} \rrbracket$, and hence gives a closed immersion $\jmath: \mathcal{W} \rightarrow \mathcal{W}^{\prime}$. Define a homomorphism $\sigma: \mathbf{T}^{\prime} \rightarrow \mathbf{T}$ of $\mathbb{Q}_{p}$-algebras as follows: for a prime $\mathfrak{l}$ of $F$, define

(1) $\sigma\left(T_{\mathfrak{l}_{1}}\right)=\sigma\left(T_{\mathfrak{l}_{2}}\right)=T_{\mathfrak{l}}$, if $\mathfrak{l}$ splits as $\mathfrak{l O}_{F^{\prime}}=\mathfrak{l}_{1} \cdot \mathfrak{l}_{2}$ in $F^{\prime}$;

(2) $\sigma\left(T_{\mathfrak{l}^{\prime}}\right)=T_{\mathfrak{l}}^{2}-2 l S_{\mathfrak{l}}$, if $\mathfrak{l}$ is inert in $\mathcal{O}_{F^{\prime}}$ and set $\mathfrak{l}^{\prime}=\mathfrak{l} \mathcal{O}_{F^{\prime}}$;

(3) $\sigma\left(T_{\mathfrak{l}^{\prime}}\right)=T_{\mathfrak{l}}$, if $\mathfrak{l}$ is ramified in $F^{\prime}$, and let $\mathfrak{l}^{\prime}$ be the unique prime of $F^{\prime}$ over $\mathfrak{l}$.

Applying Theorem 6.1.4 to the above datum, we have the following theorem.

Theorem 6.2.2. There is a morphism $i_{F^{\prime} / F}: \mathcal{X}_{\mathrm{GL}_{2 / F}}(\mathfrak{n}) \rightarrow \mathcal{X}_{\mathrm{GL}_{2 / F^{\prime}}}\left(\mathfrak{n}^{\prime}\right)$ interpolating the quadratic base change from $F$ to $F^{\prime}$ on non-critical classical points.

To apply the above theorem to Hilbert modular eigenvarieties, we need to make some explicit computations. Let $I^{\prime}=\operatorname{Hom}(F, \overline{\mathbb{Q}})=\operatorname{Hom}\left(F, \overline{\mathbb{Q}}_{p}\right)$. We identify the set $I$ (resp. $I^{\prime}$ ) with the set of primes of $F$ (resp. $F^{\prime}$ ) over $p$. Since $p$ splits completely in $F^{\prime}$, every prime $i$ in $I$ splits into two primes in $I^{\prime}$, and we use $i_{1}^{\prime}$ and $i_{2}^{\prime}$ to denote the two primes. Under the natural map $\mathcal{O}_{\mathfrak{p}_{i}} \rightarrow \mathcal{O}_{\mathfrak{p}_{i_{j}^{\prime}}}^{\prime}$, the image $\pi_{i_{j}^{\prime}}$ of $\pi_{i}$ is a uniformizer of $\mathcal{O}_{\mathfrak{p}_{i_{j}^{\prime}}^{\prime}}^{\prime}$, for $j=1,2$. As in 4.1 . we can use these $\pi_{i^{\prime}}{ }^{\prime}$ 's for all $i \in I$ and $j=1,2$ to define a full set $\left\{\left(T_{i_{j}^{\prime}}\right)_{i \in I, j=1,2}, T\right\}$ of parameters on the weight space $\mathcal{W}^{\prime}$. Under these notations, the closed immersion $\jmath: \mathcal{W} \rightarrow \mathcal{W}^{\prime}$ defined above can be described in term of parameters: if $x \in \mathcal{W}\left(\mathbb{C}_{p}\right)$ has parameters $\left(\left(w_{i}\right)_{i \in I}, w_{0}\right)$, and $\jmath(x) \in \mathcal{W}^{\prime}\left(\mathbb{C}_{p}\right)$ has parameters $\left(\left(w_{i^{\prime}}\right)_{i^{\prime} \in I^{\prime}}, w_{p}^{\prime}\right)$, then $w_{i_{1}^{\prime}}=w_{i_{2}^{\prime}}=w_{i}$ for all $i \in I$ and $w_{0}=w_{0}^{\prime}$. In particular, for any $r=\left(r_{i}\right)_{i \in I} \in \mathcal{N}^{I}$, if we define $r^{\prime}=\left(r_{i^{\prime}}\right)_{i^{\prime} \in I^{\prime}} \in \mathcal{N}^{I^{\prime}}$ by $r_{i_{1}^{\prime}}=r_{i_{2}^{\prime}}=r_{i}$ for all $i \in I$, then $\jmath^{-1}\left(\mathcal{W}^{\prime>r^{\prime}}\right)=\mathcal{W}^{>r}$. 
For any closed point $x^{\prime}$ of $\mathcal{X}_{\mathrm{GL}_{2 / F^{\prime}}}\left(\mathfrak{n}^{\prime}\right)$, recall that we use $a_{i^{\prime}}(x)$ to denote its corresponding $U_{\pi_{i^{\prime}}}$-eigenvalue for all $i^{\prime} \in I^{\prime}$. From the explicit description of the homomorphism $\sigma: \mathbf{T}^{\prime} \rightarrow \mathbf{T}$, we have $a_{i_{1}^{\prime}}\left(i_{F^{\prime} / F}(x)\right)=a_{i_{2}^{\prime}}\left(i_{F^{\prime} / F}(x)\right)=a_{i}(x)$ for any closed point $x$ of $\mathcal{X}_{\mathrm{GL}_{2 / F}}(\mathfrak{n})$. Combining Theorems 1.0.2, 6.2.1 and 6.2.2, we have the following description of the boundary behavior of Hilbert modular eigenvarieties:

Theorem 6.2.3. We use $\Sigma$ to denote the subset $\{0\} \bigcup\left\{1+2 k \mid k \in \mathbb{Z}_{\geqslant 0}\right\}$ of $\mathbb{Z}$. The eigenvariety $\mathcal{X}_{\mathrm{GL}_{2 / F}}(\mathfrak{n})^{>1 / p}$ is a disjoint union

$$
\mathcal{X}_{\mathrm{GL}_{2 / F}}(\mathfrak{n})^{>1 / p}=\bigsqcup_{l \in \Sigma^{I}, \sigma \in\{ \pm\}^{I}} \mathcal{X}_{l, \sigma}
$$

of (possibly empty) rigid analytic spaces which are finite over $\mathcal{W}^{>1 / p}$ via $w$, such that for each closed point $x \in \mathcal{X}_{l, \sigma}\left(\mathbb{C}_{p}\right)$ with $l=\left(l_{i}\right)_{i \in I} \in \Sigma^{I}$ and $\sigma=\left(\sigma_{i}\right)_{i \in I} \in\{ \pm\}^{I}$, we have

$$
\begin{cases}v_{p}\left(a_{i}(x)\right)=(p-1) v_{p}\left(T_{i, w(x)}\right) \cdot l_{i}, & \text { if } \sigma_{i}=-; \\ v_{p}\left(a_{i}(x)\right) \in(p-1) v_{p}\left(T_{i, w(x)}\right) \cdot\left(l_{i}, l_{i}+2\right), & \text { if } \sigma_{i}=+ \text { and } l_{i} \neq 0 ; \\ v_{p}\left(a_{i}(x)\right) \in(p-1) v_{p}\left(T_{i, w(x)}\right) \cdot(0,1), & \text { if } \sigma_{i}=+ \text { and } l_{i}=0,\end{cases}
$$

for all $i \in I$.

\section{REFERENCES}

[1] Fabrizio Andreatta, Adrian Iovita, and Vincent Pilloni. On overconvergent Hilbert modular cusp forms. Astérisque, 382:163-193, 2016.

[2] Christopher Birkbeck. The Jacquet-Langlands correspondence for overconvergent Hilbert modular forms. arXiv preprint arXiv:1610.09711, 2016.

[3] Christopher Birkbeck. Slopes of overconvergent hilbert modular forms. Experimental Mathematics, pages 1-20, 2019.

[4] Christopher D Birkbeck. Eigenvarieties associated to Hilbert modular forms. PhD thesis, University of Warwick, 2017.

[5] Kevin Buzzard. Eigenvarieties. in 'L-functions and Galois Representations', 59-120. London Math. Soc. Lecture Note Ser, 320.

[6] Kevin Buzzard. On p-adic families of automorphic forms. In Modular curves and abelian varieties, pages 23-44. Springer, 2004.

[7] Kevin Buzzard and Lloyd James Peter Kilford. The 2-adic eigencurve at the boundary of weight space. Compositio Mathematica, 141(3):605-619, 2005.

[8] Gaëtan Chenevier. Une correspondance de Jacquet-Langlands p-adique. Duke Mathematical Journal, 126(1):161194, 2005.

[9] Robert Coleman and Barry Mazur. The eigencurve. London Mathematical Society Lecture Note Series, pages 1-114, 1998.

[10] Pierre Colmez. Fonctions d' une variable p-adique. Astérisque, 330:13-59, 2010.

[11] Matthew Emerton. Completed cohomology and the p-adic langlands program. In Proceedings of the International Congress of Mathematicians, volume 2, pages 319-342, 2014.

[12] David Hansen and James Newton. Universal eigenvarieties, trianguline Galois representations, and p-adic Langlands functoriality. Journal für die reine und angewandte Mathematik, 2017(730):1-64, 2017.

[13] Haruzo Hida. Galois representations into $\mathrm{GL}_{2}\left(\mathbb{Z}_{p} \llbracket X \rrbracket\right)$ attached to ordinary cusp forms. Inventiones mathematicae, 85(3):545-613, 1986.

[14] Haruzo Hida. Iwasawa modules attached to congruences of cusp forms. In Annales scientifiques de l'École Normale Supérieure, volume 19, pages 231-273, 1986.

[15] Haruzo Hida. On p-adic Hecke algebras for over totally real fields. Annals of mathematics, pages 295-384, 1988.

[16] Daniel Jacobs. Slopes of compact Hecke operators, volume 2. Citeseer, 2003.

[17] Christian Johansson and James Newton. Parallel weight 2 points on hilbert modular eigenvarieties and the parity conjecture. arXiv preprint arXiv:1801.04719, 2018.

[18] Christian Johansson and James Newton. Extended eigenvarieties for overconvergent cohomology. Algebra 63 Number Theory, 13(1):93-158, 2019.

[19] Kiran S Kedlaya. p-adic Differential Equations, volume 125. Cambridge University Press, 2010.

[20] Ruochuan Liu, Daqing Wan, and Liang Xiao. The eigencurve over the boundary of weight space. Duke Mathematical Journal, 166(9):1739-1787, 2017.

[21] Barry Mazur. Deforming galois representations. In Galois Groups over $\mathbb{Q}$, pages 385-437. Springer, 1989.

[22] James S Milne. Étale cohomology (PMS-33), volume 33. Princeton university press, 2016. 
[23] Rufei Ren, Daqing Wan, Liang Xiao, and Myungjun Yu. Slopes for higher rank artin-schreier-witt towers. Transactions of the American Mathematical Society, 370(9):6411-6432, 2018.

[24] Jean-Pierre Serre. Formes modulaires et fonctions zêta p-adiques. In Modular functions of one variable III, pages 191-268. Springer, 1973.

[25] Yichao Tian and Liang Xiao. Arithmétique p-adique des formes de hilbert p-adic cohomology and classicality of overconvergent hilbert modular forms. Astérisque, 382:73-162, 2016.

[26] Atsushi Yamagami. On p-adic families of Hilbert cusp forms of finite slope. Journal of Number Theory, 123(2):363-387, 2007.

[27] Lynnelle Ye. Slopes in eigenvarieties for definite unitary groups. preprint available at http: //www. math. harvard. edu/ lynnelle/lynnelle-ye-draft.pdf 2019

Department of Mathematics, Fudan University, 220 Handan Rd., Yangpu District, Shanghai 200433, CHINA.

Email address: rufeir@fudan.edu.cn

Bin Zhao, Morningside Center of Mathematics, Academy of Mathematics and Systems Science, Chinese Academy of Sciences, University of the Chinese Academy of Sciences Beijing, 100190, China.

Email address: bin.zhao@amss.ac.cn 